\title{
Dynamic Complexation of Solutes in Capillary Electrophoresis
}

by

MICHAEL T. BOWSER

Hons. B.Sc., Dalhousie University, 1994

A Thesis Submitted in Partial Fulfillment of the Requirements for the Degree of

DOCTOR OF PHILOSOPHY

in

The Faculty of Graduate Studies

Department of Chemistry

We accept this thesis as conforming to the required standard

THE UNIVERSITY OF BRITISH COLUMBIA

September 1998

(C) Michael T. Bowser, 1998 
in presenting this thesis in partial fulfilment of the requirements for an advanced degree at the University of British Columbia, I agree that the Library shall make it freely available for reference and study. I further agree that permission for extensive copying of this thesis for scholarly purposes may be granted by the head of my department or by his or her representatives. It is understood that copying or publication of this thesis for financial gain shall not be allowed without my written permission.

Department of Chemistry

The University of British Columbia Vancouver, Canada

Date Oct.5,1998 


\section{Abstract}

A general theory that describes analyte migration behavior in chromatography, electrophoresis, and ultracentrifugation is described. The theory accounts for multiple stoichiometry and multiple additive interactions through the use of individual capacity factors. The unified separation theory includes a general expression for resolution which shows that the resolution of a pair of analytes is linearly related to the ratio of the linear velocities of the two analytes. A CE separation with the presence of both 1:1 and 1:2 stoichiometry analyte-additive interactions is described using the general separation theory. The effect of the higher order interactions on the mobility of the analyte and methods for detecting deviations from the 1:1 model are discussed. Multivariate binding isotherms are used to describe the effect of multiple equilibria on analyte mobility. The effect of secondary interactions on the shapes of these curves is determined. The general theory is especially useful in describing hybrid techniques such as capillary electrochromatography.

The statistical methods for estimating equilibrium constants from binding experiments are compared using Monte Carlo analyses. Simulated experiments with realistic error assumptions are used to compare the accuracy and precision of the nonlinear regression to the three linear plotting methods. The effect of the additive concentration range and the maximum response range was demonstrated. It was shown that binding constants estimated using nonlinear regression were generally more accurate and more precise than constants estimated using one of the linear transformations. The effect of experimental conditions on the precision of the binding constant estimates was shown to be related to the curvature in the binding isotherm.

The solvent can have an important effect on analyte-additive interactions in CE. An assay for an oligomeric porphyrin anti-cancer drug was used as an example of nonaqueous $\mathrm{CE}$ separations. It was shown that the dipolar interaction between the porphyrins and a nonionic polyether surfactant (brij 35) could be used to improve separation selectivity. A group of 
monomeric porphyrin acids was studied to gain insight into the mechanism of the brij 35porphyrin interaction in nonaqueous buffers. It was proposed that brij 35 interacts with the carboxyl groups on the porphyrins through dipolar interactions. It was also suggested that the orientation of porphyrin acids in the electric field might have an effect on their mobilities. 


\section{Table of Contents}

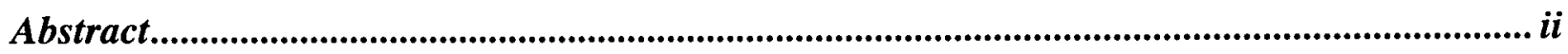

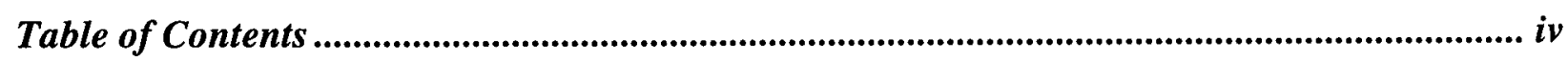

List of Tables .......................................................................................................................... vii

List of Figures .............................................................................................................................. viii

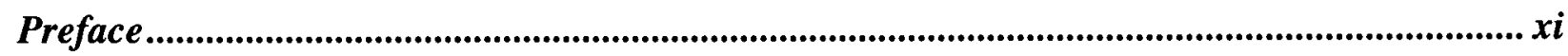

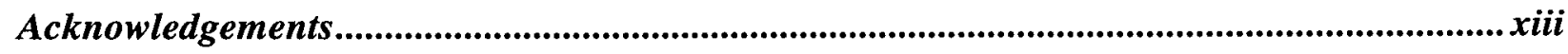

Part 1: Unified Separation Science ............................................................................................. 1

1.1 Previous Strategies for Unifying Separation Science .............................................................................. 1

1.1.1 Introduction .................................................................................................................

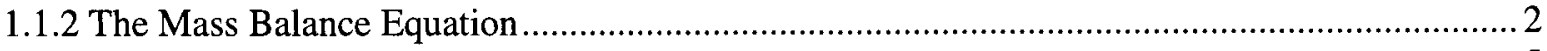

1.1.3 Virtual Migration Distances ………………………........................................................

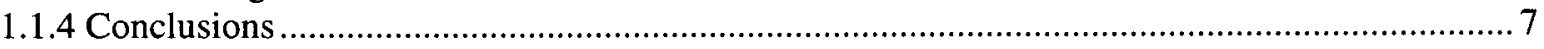

1.2 A Unified Theory for Separation Science........................................................................................................8

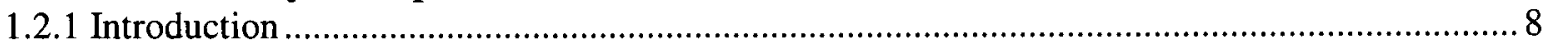

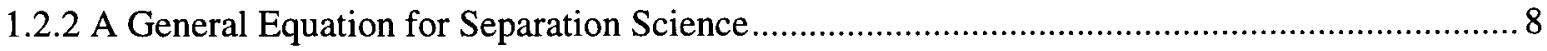

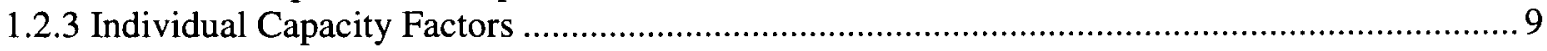

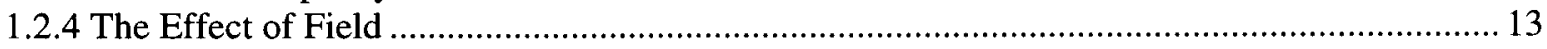

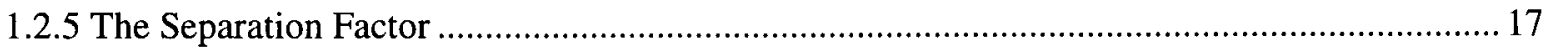

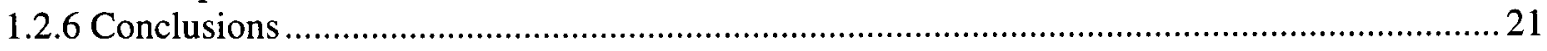

1.3 Higher Order Equilibria and Their Effect on Analyte Migration Behavior in Capillary

Electrophoresis ............................................................................................................................................... 23

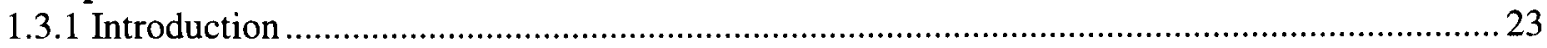

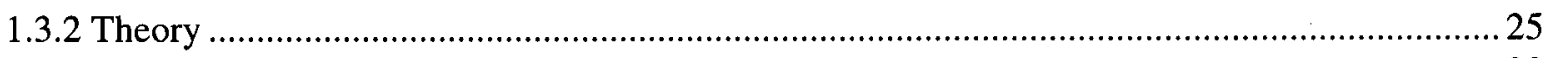

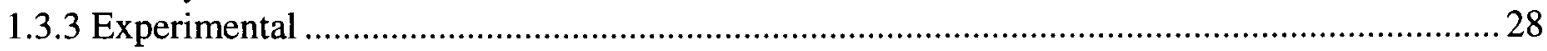

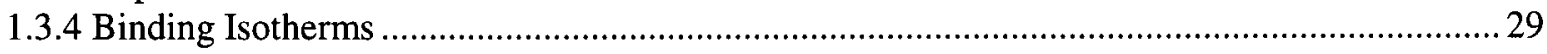

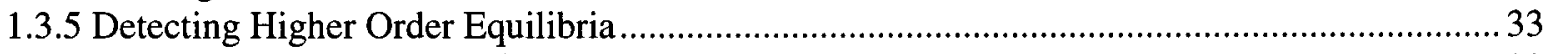

1.3.6 Additive Concentration Range ……………………............................................................. 39

1.3.7 Capacity Factors and the Fractions of Analyte Species ...........................................................4. 42

1.3.8 Predicting the Electroosmotic Mobility and Analyte Migration Times ......................................45

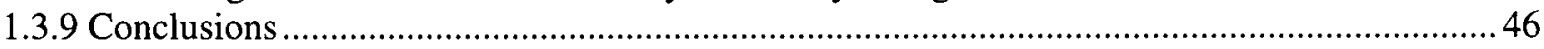

1.4 The Properties of Multivariate Binding Isotherms in Capillary Electrophoresis ........................4 48

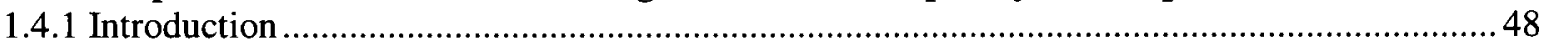

1.4.2 First Order Binding in the Presence of Two Additives .............................................................48

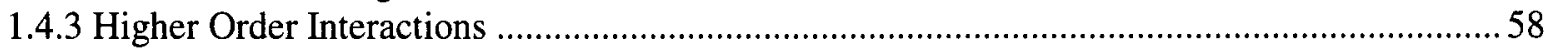

1.4.4 Interactions Between Additives .......................................................................................6

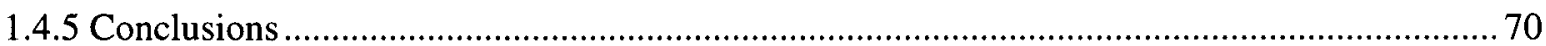

1.5 The Effect of Complexation Additives on Analyte Migration Behavior in Capillary Electrochromatography ..........................................................................................................................72

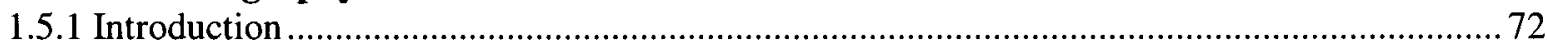

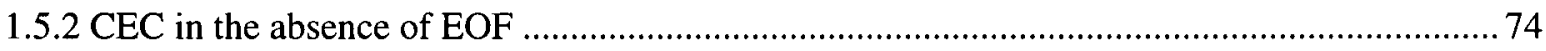

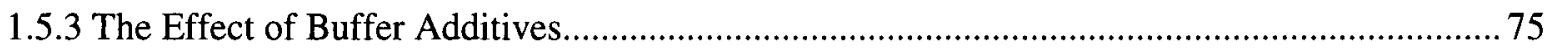

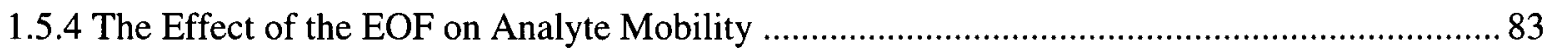

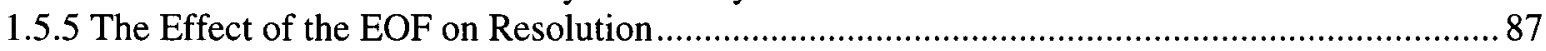

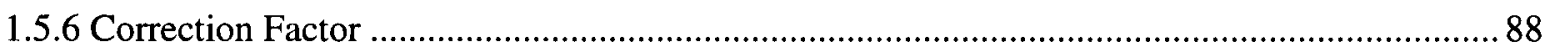

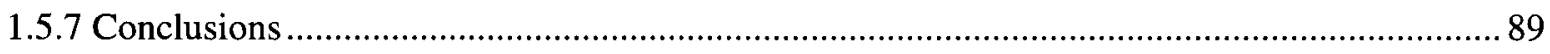




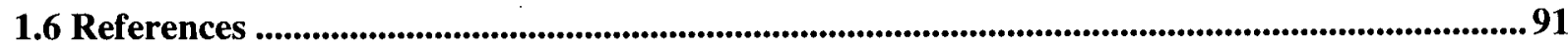

Part 2: Monte Carlo Simulation of Error Propagation in the Determination of Binding Constants from Rectangular Hyperbolae...................................................................................... 94

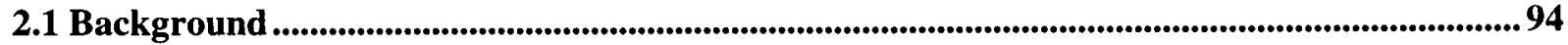

2.2 The Ligand Concentration Range

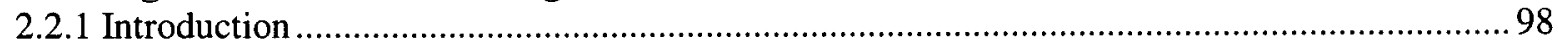

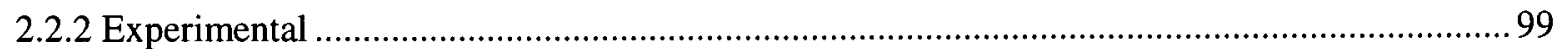

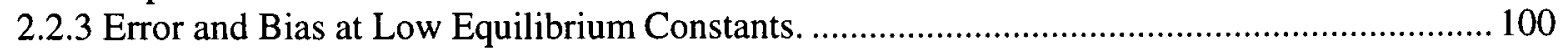

2.2.4 Error and Bias at High Equilibrium Constants............................................................... 103

2.2.5 Comparing the Relative 95\% Ranges at Low K and High K....................................... 105

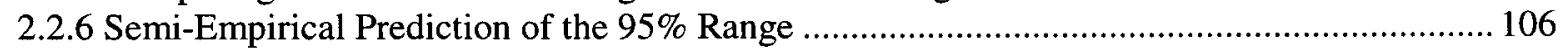

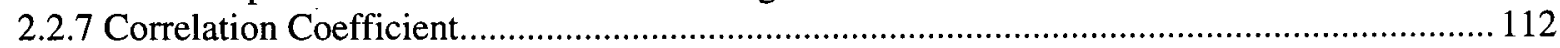

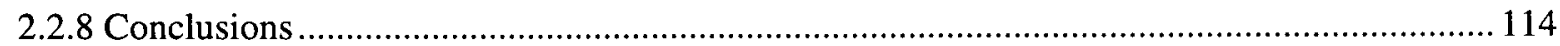

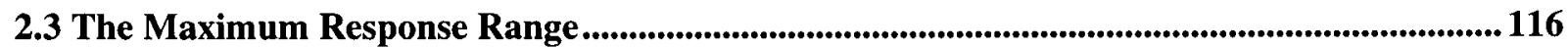

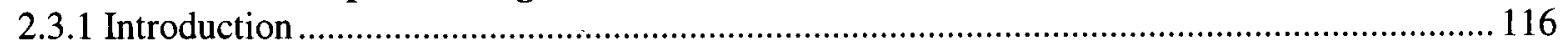

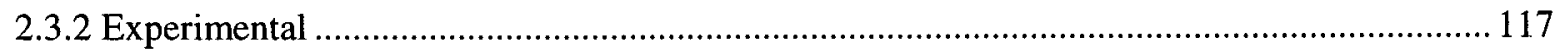

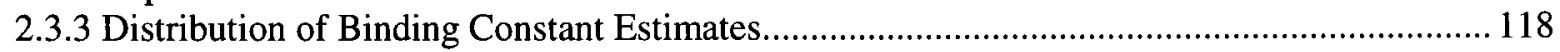

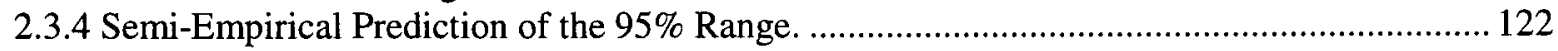

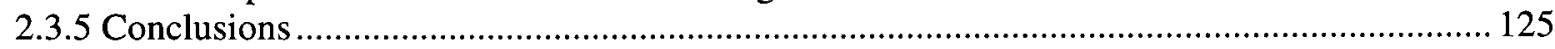

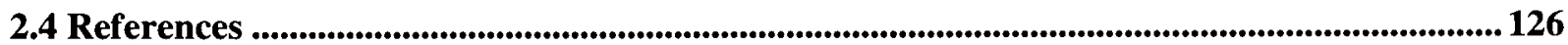

Part 3: Dynamic Complexation Capillary Electrophoresis in Nonaqueous Media ............... 128

3.1 Analyte-Additive Interactions in Nonaqueous Solvents..................................................................128

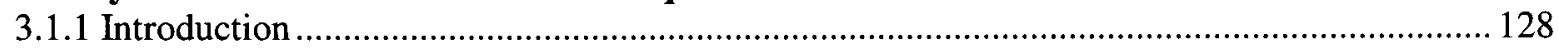

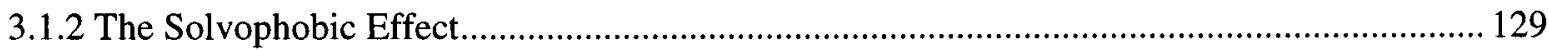

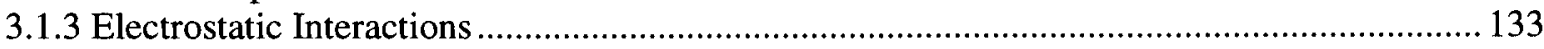

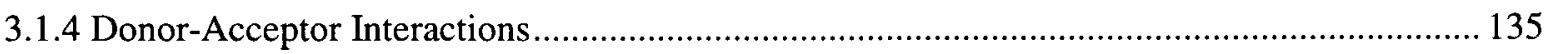

3.1.5 The Complexity of Analyte-Additive Interactions in Nonaqueous CE.............................. 140

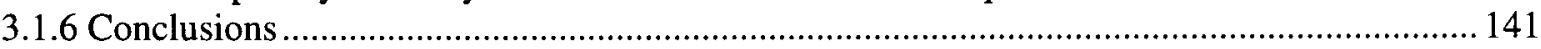

3.2 Development and Application of a Nonaqueous Capillary Electrophoresis System for the Analysis of Porphyrins and Their Oligomers (PHOTOFRIN ${ }^{\circledR}$ )....................................................143

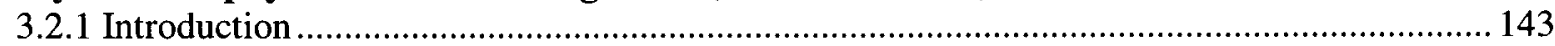

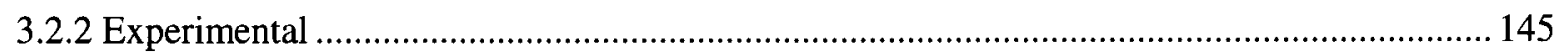

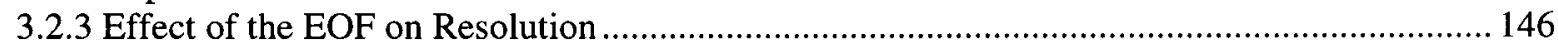

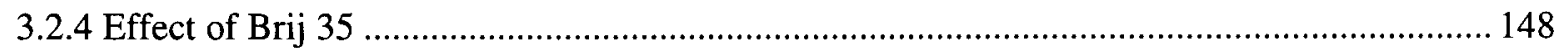

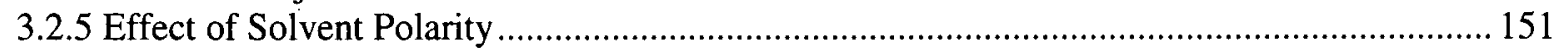

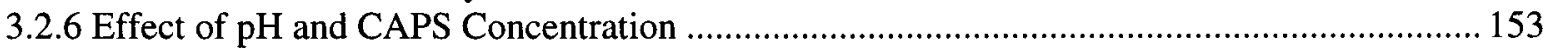

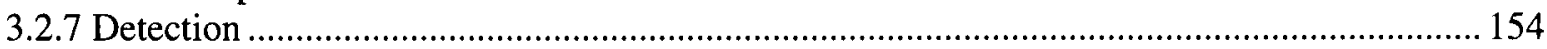

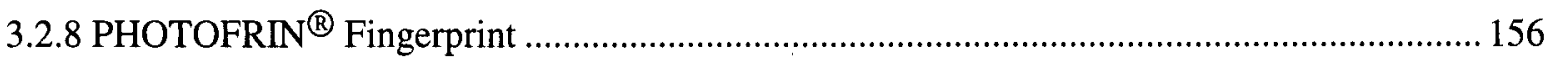

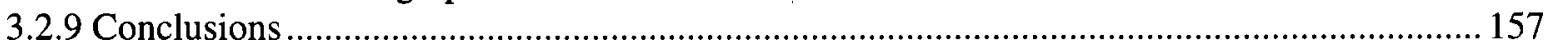


3.3 Quantitative Description of the Migration Behavior of Porphyrins in a Nonaqueous Capillary

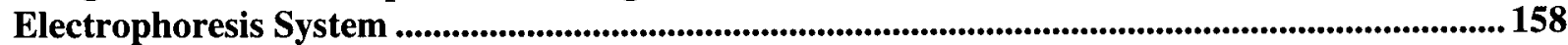

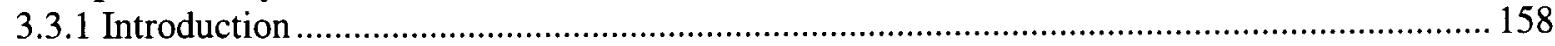

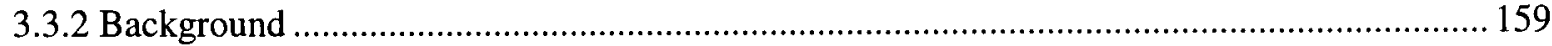

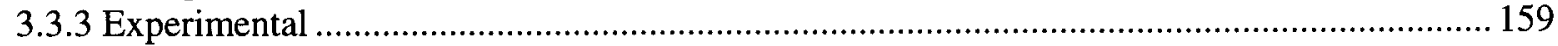

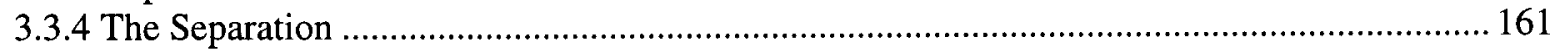

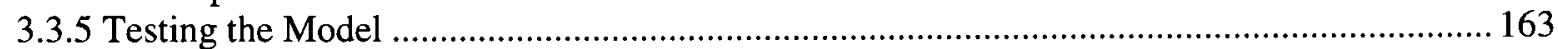

3.3.6 Estimating the Binding Constant and Complex Mobility .............................................. 165

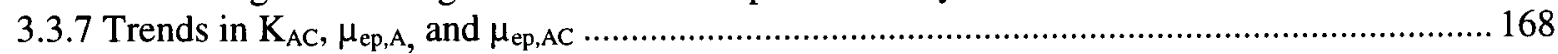

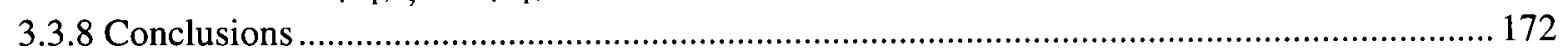

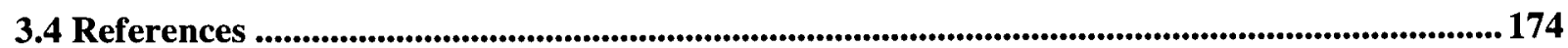

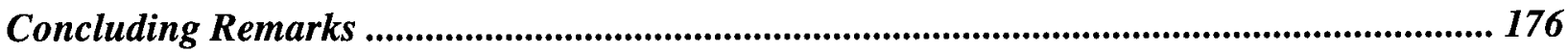

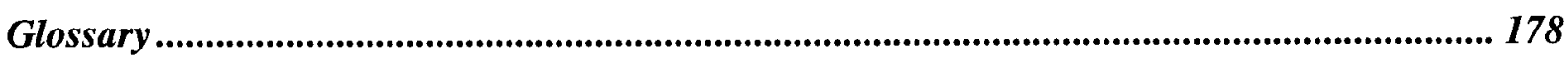

Appendix I: Nonlinear Least-Squares Analysis ............................................................ 179

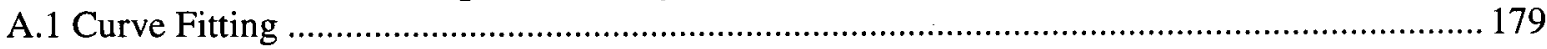

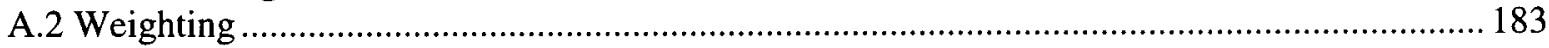

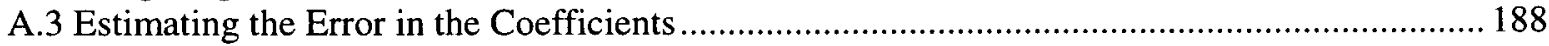

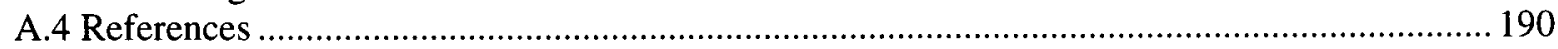




\section{List of Tables}

Table 1.2.1 Fundamental equations in the major areas of separation science

Table 1.3.1 Equilibrium constants and electrophoretic mobilities calculated for 4-phenylphenol, 4,4'-biphenol, and phenol using equations assuming a 1:1 or 1:1 and $1: 2$ complexation stiochiometry

Table 1.3.2 Microscopic and macroscopic equilibrium constants used to generate the curves in Figures 1.3.3 and 1.3.4 34

Table 2.1.1 Nonlinear and linear forms of the binding isotherm in CE. .97

Table 2.3.1 Equations and maximum response ranges for absorbance, NMR, MichaelisMenten kinetics and capillary electrophoresis

Table 3.1.1 Physical properties affecting analyte-additive interactions in solvents commonly used in CE.

Table 3.3.1 Binding constants for the porphyrin-brij 35 complexes

Table 3.3.2 Electrophoretic mobilities of the uncomplexed porphyrins and the porphyrinbrij 35 complexes 


\section{List of Figures}

Figure 1.1.1 Representation of the approach used to derive the mass balance equation..... 3

Figure 1.2.1 Effect of $k_{\mathrm{B}}^{\prime}$ on Resolution . 18

Figure 1.2.2 The effect of the EOF on resolution. 21

Figure 1.3.1 Equilibria between an analyte with two binding sites and an additive with one binding site. 25

Figure 1.3.2 Binding isotherms for 4-phenylphenol, 4,4'-biphenol, and phenol. 31

Figure 1.3.3 Hypothetical binding isotherms calculated using the equilibrium constants listed in Table 1.3.2.

Figure 1.3.4 $x$-Reciprocal plots, $y$-reciprocal plots, and double reciprocal plots of the binding isotherms in Figure 1.3.3.

Figure 1.3.5 $x$-Reciprocal plots, $y$-reciprocal plots, and double reciprocal plots for 4phenylphenol, 4,4'-biphenol, and phenol

Figure 1.3.6 Comparison of the capacity factors and fractions of free, singly bound, and doubly bound 4-phenylphenol for 0 to $250 \mathrm{mM} \mathrm{HP}-\beta-\mathrm{CD}$.

Figure 1.3.7 The migration times predicted using the calculated electrophoretic mobilities, viscosity correction factors, and electroosmotic mobilities for 4-phenylphenol, 4,4'-biphenol, phenol, and the EOF marker

Figure 1.4.1 Three-dimensional plots of a binding isotherm surface where both additives interact with the analyte with 1:1 binding stoichiometries.

Figure 1.4.2 Contour plots of a binding isotherm surface where both additives interact with the analyte with $1: 1$ binding stoichiometries.

Figure 1.4.3 Profile plots of a binding isotherm surface where both additives interact with $1: 1$ binding stoichiometries

Figure 1.4.4 Contour plot of a binding isotherm surface where additive $\mathrm{C}$ interacts with both 1:1 and 1:2 stoichiometries and additive $D$ has a 1:1 binding stoichiometry...

Figure 1.4.5 Profile plots of a binding isotherm surface where additive $\mathrm{C}$ interacts with both $1: 1$ and 1:2 stoichiometries and additive $D$ interacts with a 1:1 binding stoichiometry

Figure 1.4.6 Profile plots of a binding isotherm surface where additive $\mathrm{C}$ interacts with both $1: 1$ and 1:2 stoichiometries and additive $D$ interacts with a 1:1 binding stoichiometry

Figure 1.4.7 Profile plots of a binding isotherm surface where additive $C$ interacts with a 1:2 stoichiometry and additive D has a 1:1 binding stoichiometry

Figure 1.4.8 Profile plots of a binding isotherm surface where interactions between the additives invalidate the assumptions $[C]=[C]_{0}$ and $[D]=[D]_{0}$

Figure 1.5.1 Equilibria describing CEC with a complexation additive present in the buffer......

Figure 1.5.2 The effect of the additive concentration on the capacity factors and the fraction of analyte present as each species for a system described by the equilibrium shown in Figure 1.5.1.

Figure 1.5.3 The effect of $k_{\mathrm{A}, \mathrm{S}}^{\prime}$ on the binding isotherms of an interaction between an analyte and an additive in a CEC system.

Figure 1.5.4 The apparent binding constant, the apparent analyte-additive complex mobility, and the apparent uncomplexed analyte mobility over a range of values for $k_{\mathrm{A}, \mathrm{S}}^{\prime}$ 
Figure 1.5.5 The effect of $\mathrm{K}_{\mathrm{AC,S}}\left(\mathrm{V}_{\mathrm{S}} / \mathrm{V}_{\mathrm{M}}\right)$ on the binding isotherms of an interaction between an analyte and an additive in a CEC system ....

Figure 1.5.6 The apparent binding constant, the apparent analyte-additive complex mobility, and the apparent uncomplexed analyte mobility over a range of values for $\mathrm{K}_{\mathrm{AC}, \mathrm{s}}\left(\mathrm{V}_{\mathrm{S}} / \mathrm{V}_{\mathrm{M}}\right)$

Figure 2.2.1 Binding isotherms for a set range of additive (ligand) concentrations at $\mathrm{K}=$ $1 \mathrm{M}^{-1}, 3.125 \mathrm{M}^{-1}, 15 \mathrm{M}^{-1}, 50 \mathrm{M}^{-1}, 200 \mathrm{M}^{-1}, 800 \mathrm{M}^{-1}$, and $4000 \mathrm{M}^{-1}$.

Figure 2.2.2 The distributions of the binding constant estimates calculated using double reciprocal, $y$-reciprocal, $x$-reciprocal, and nonlinear regression methods over $\mathrm{K}=1$ to $50 \mathrm{M}^{-1}$

Figure 2.2.3 The distributions of the binding constant estimates calculated using double reciprocal, $y$-reciprocal, $x$-reciprocal, and nonlinear regression methods over $\mathrm{K}=50$ to $4000 \mathrm{M}^{-1}$

Figure 2.2.4 The relative 95\% ranges of the binding constants estimated using the double reciprocal, weighted double reciprocal, $y$-reciprocal, weighted $y$-reciprocal, $x$-reciprocal, weighted $x$-reciprocal, and nonlinear regression methods for $\mathrm{K}=1$ to $50 \mathrm{M}^{-1}$, and $\mathrm{K}=50$ to $4000 \mathrm{M}^{-1}$.

Figure 2.2.5 The change in slope, information content, and dispersion of $\mathrm{G}$ for binding isotherms with $\mathrm{K}=1$ to $800 \mathrm{M}^{-1}$ and a set range of additive (ligand) concentrations

Figure 2.2.6 Correlations between the relative $95 \%$ ranges of the binding constants estimated using the nonlinear regression method and (a) $(\Delta \text { slope })^{-1}$, (b) $(\Sigma \mathrm{I}(\mathrm{p}))^{-1}$, and (c) $\left(\Sigma\left(\mathrm{G}_{\mathrm{i}}-\mathrm{G}_{\text {mean }}\right)^{2}\right)^{-1}$ for $\mathrm{K}=1$ to $50 \mathrm{M}^{-1}$ and $\mathrm{K}=50$ to $4000 \mathrm{M}^{-1}$.

Figure 2.2.7 The median correlation coefficients $\left(\mathrm{R}^{2}\right)$ for the double reciprocal, weighted double reciprocal, $y$-reciprocal, weighted $y$-reciprocal, $x$-reciprocal, weighted $x$ reciprocal, and nonlinear regression methods for $\mathrm{K}=1$ to $20 \mathrm{M}^{-1}$, and $\mathrm{K}=50$ to 4000 $M^{-1}$

Figure 2.3.1 Binding isotherms with different maximum response ranges

Figure 2.3.2 Distributions of the binding constants estimated using the double reciprocal, $y$-reciprocal, $x$-reciprocal, and nonlinear regression methods for $\left(\mu_{\mathrm{ep}, \mathrm{AC}}-\mu_{\mathrm{ep}, \mathrm{A}}\right)=0$ to $15 \times 10^{-5} \mathrm{~cm}^{2} \mathrm{~V}^{-1} \mathrm{~s}^{-1}$

Figure 2.3.3 The 95\% ranges of binding constants estimated using the double reciprocal, weighted double reciprocal, $y$-reciprocal, weighted $y$-reciprocal, $x$-reciprocal, weighted $x$-reciprocal, and nonlinear regression methods for $\left(\mu_{\mathrm{ep}, \mathrm{AC}}-\mu_{\mathrm{ep}, \mathrm{A}}\right)=0$ to $15 \times 10^{-5} \mathrm{~cm}^{2} \mathrm{~V}^{-1} \mathrm{~s}^{-1}$.

Figure 2.3.4 Bias in the distribution of binding constants estimated using the double reciprocal, weighted double reciprocal, $y$-reciprocal, weighted $y$-reciprocal, $x$-reciprocal, weighted $x$-reciprocal, and nonlinear regression methods for $\left(\mu_{\mathrm{ep}, \mathrm{AC}}-\mu_{\mathrm{ep}, \mathrm{A}}\right)=0$ to $5 \times 10^{-5}$ $\mathrm{cm}^{2} \mathrm{~V}^{-1} \mathrm{~s}^{-1}$

Figure 2.3.5 (a) Correlation between the relative 95\% ranges of binding constants estimated using the nonlinear regression method and the reciprocal of the difference between the slope at the lowest and highest additive concentration. (b) Comparison between the 95\% ranges of the binding constants estimated using the nonlinear regression method and the curve predicted using the correlation shown in (a).

Figure 3.1.1 Structures of several additives used to modify analyte migration behavior in nonaqueous $\mathrm{CE}$

Figure 3.2.1 The oligomerization reaction of the porphyrin monomers. Both the monomers and the oligomers are present in PHOTOFRIN ${ }^{\circledR}$ 
Figure 3.2.2 Effect of varying brij 35 concentration on the nonaqueous CE separation of PHOTOFRIN ${ }^{\circledR}$

Figure 3.2.3 Effect of varying water content on the separation of PHOTOFRIN ${ }^{\circledR}$

Figure 3.2.4 Plot of separation efficiency (theoretical plate number) vs. buffer water content $(\% \mathrm{v} / \mathrm{v})$ for the porphyrin monomers

Figure 3.2.5 Comparison of absorbance and fluorescence detection for the nonaqueous CE separation of PHOTOFRIN® 155

Figure 3.2.6 Electropherograms of three different batches of PHOTOFRIN ${ }^{\circledR}$ 156

Figure 3.2.7 Qualitative comparisons of stressed PHOTOFRIN ${ }^{\circledR}$ samples. 157

Figure 3.3.1 The structures of the porphyrin acids studied 161

Figure 3.3.2 Electropherograms of the six porphyrin acids at different brij 35 concentrations 162

Figure 3.3.3 The $x$-reciprocal plots of the six porphyrin acids 164

Figure 3.3.4 Nonlinear fits of the binding isotherms of the six porphyrin acids 166

Figure 3.3.5 Comparison of the mobilities and migration times estimated using the constants in Tables 3.3.1 and 3.3.2, and the correlation between the viscosity correction factor and the brij 35 concentration to the experimental data

Figure 3.3.6 Binding constants of the porphyrin-brij 35 complexes estimated using nonlinear regression

Figure 3.3.7 Electrophoretic mobilities of the uncomplexed porphyrins $\left(\mu_{\mathrm{ep}, \mathrm{A}}\right)$ and the porphyrin-brij 35 complexes $\left(\mu_{\text {ep.Ac }}\right)$

Figure 3.3.8 Preferred orientations of uncomplexed and complexed, mesoporphyrin and the type I porphyrins in the electric field 


\section{Preface}

Over the past several years capillary electrophoresis (CE) has matured as an analytical separation technique. One of the areas that has experienced the most success is the use of complexation additives in the separation buffer. Introducing additional equilibria into the separation system provides the potential for improved selectivity. Later it was realized that $\mathrm{CE}$ could be used not only to separate compounds, but also as a tool to study the equilibria formed between the analyte and the additives.

This thesis deals with the fundamental aspects of equilibria in CE. The first section develops the theory necessary to describe the complicated equilibria that often exist in CE. Models that only accounted for 1:1 interactions with a single additive were extended to account for multiple stoichiometry or multiple additive systems. In developing these theories we realized that they apply not only in CE, but separation science in general.

The second part of this thesis deals with the statistical methods used to estimate binding constants from experimental data. Unless the methods used to estimate binding constants are shown to be valid, it is impossible to determine the significance of the estimated binding constants.

The third part of this thesis discusses equilibria in nonaqueous CE systems. Nonaqueous $\mathrm{CE}$ is one of the fastest growing areas of separation science. This, combined with the fact that equilibria are often more complicated in nonaqueous solvents, makes nonaqueous $\mathrm{CE}$ an excellent application of the theories developed in the previous sections.

Portions of the following sections have been previously published elsewhere:

$1.1 \quad$ M. T. Bowser and D. D. Y. Chen. "Recent Developments Towards a Unified Theory for Separation Science." Electrophoresis 1998, 19, 1586-1589. (review)

1.2 M. T. Bowser, G. M. Bebault, X. Peng, and D. D. Y. Chen. "Redefining the Separation Factor: A Potential Pathway to a Unified Separation Science." Electrophoresis 1997, 18, 2928-2934. 
$1.3 \quad$ M. T. Bowser and D. D. Y. Chen. "Higher Order Equilibria and Their Effect on Analyte Migration Behavior in Capillary Electrophoresis." Anal. Chem. 1998, 70, 3261-3270.

1.4 M. T. Bowser, A. R. Kranack and D. D. Y. Chen. "The Properties of Multivariate Binding Isotherms in Capillary Electrophoresis." Anal. Chem. 1998, 70, 1076-1084.

1.5 M. T. Bowser and D. D. Y. Chen. "The Effect of Complexation Additives on Analyte Migration Behavior in Capillary Electrochromatography." Electrophoresis 1998, 19, $1452-1460$.

2.1,2.2 M. T. Bowser and D. D. Y. Chen. "Monte Carlo Simulation of Error Propagation in the Determination of Binding Constants from Rectangular Hyperbolae: The Ligand Concentration Range and the Binding Constant." J. Phys. Chem. A, in press.

2.3 M. T. Bowser and D. D. Y. Chen. "Monte Carlo Simulation of Error Propagation in the Determination of Binding Constants from Rectangular Hyperbolae: The Effect of the Maximum Response Range" J. Phys. Chem. A, in press.

3.1 M. T. Bowser, A. R. Kranack and D. D. Y. Chen. "Analyte-Additive Interactions in Nonaqueous Capillary Electrophoresis: A Critical Review." TrAC, Trends Anal. Chem. 1998, 17, 424-434. (review)

3.2 M. T. Bowser, E. D. Sternberg, and D. D. Y. Chen. "Development and Application of a Nonaqueous Capillary Electrophoresis System for the Analysis of Porphyrins and Their Oligomers (PHOTOFRIN)." Anal. Biochem. 1996, 241, 143-150.

3.3 M. T. Bowser, E. D. Sternberg, and D. D. Y. Chen. "Quantitative Description of Migration Behavior of Porphyrins Based on the Dynamic Complexation Model in a Nonaqueous Capillary Electrophoresis System." Electrophoresis, 1997, 18, 82-91. 
xiii.

\section{Acknowledgements}

I wish to thank the National Sciences and Engineering Research Council of Canada (NSERC) for funding this research as well as my post-graduate fellowship. The University of British Columbia also provided a University Graduate Fellowship. QLT Phototherapeutics Inc. supplied the PHOTOFRIN ${ }^{\circledast}$ and the porphyrin standards. Some of the CE data was recorded on an automated instrument kindly loaned from Beckman Instruments.

I want to thank Dr. David Chen for providing an environment which encouraged a successful research program, my parents for their belief in me, and my wife Charlotte for all of her support over the past four years. 


\section{Part 1: Unified Separation Science}

\subsection{Previous Strategies for Unifying Separation Science}

\subsubsection{Introduction}

Modern separation science, based on differential migration methods, originated when Mikhail Tswett separated plant extracts into a series of coloured bands on a bed of calcium carbonate in $1903^{(1)}$. After years of relative obscurity, chromatography became a truly analytical technique with the introduction of liquid-liquid chromatography by Martin and Synge in $1941^{(2)}$ and gas-liquid chromatography by James and Martin in $1951^{(3)}$. Other separation techniques, taking advantage of different types of fields, were also being developed early this century at Uppsala University in Sweden ${ }^{(4)}$. Svedberg studied the sedimentation of proteins and metal colloids under the influence of strong centrifugal fields ${ }^{(5)}$, and Tiselius, a student of Svedberg, studied moving boundary electrophoresis ${ }^{(6)}$. Hjertén demonstrated zone separation of analytes in small tubes by carrier free electrophoresis in 1967(7). Jorgenson and Lukacs at the University of North Carolina later demonstrated capillary electrophoresis (CE) with a glass capillary and a fluorescence detector ${ }^{(8,9)}$.

Chromatography, electrophoresis, and ultracentrifugation have emerged as the three major differential migration methods of separation science. Although there have been many modifications of these techniques, until recently it was not difficult to classify, and therefore determine which theory to apply to, a particular separation method. The development of hybrid techniques such as field flow fractionation $(\mathrm{FFF})^{(10-12)}$, micellar electrokinetic capillary chromatography (MECC) $)^{(13,14)}$, and capillary electrochromatography $(\mathrm{CEC})^{(9,15,16)}$ have made

classification less straightforward. Initially, combinations of existing theories were developed to describe hybrid techniques. While this approach is useful, it would be more satisfying if all separation techniques could be described according to one generalized theory. Strain et al.(17,18) pointed out that a common characteristic of differential migration methods is the movement of 
the analyte. The reviews of these differential migration methods classified different techniques according to the type of force (e.g. hydrostatic, centrifugal, electric, etc.) experienced by the analyte. Giddings' description of a unified separation science stressed many of the common thermodynamic characteristics of different techniques ${ }^{(19-22)}$. The following sections summarize some of the recent publications that describe chemical separation methods according to a unified theory.

\subsubsection{The Mass Balance Equation}

The mass balance equations describing the velocity of a boundary in electrophoresis were initially developed by Longsworth ${ }^{(23)}$. Wilson first applied the mass balance equation to chromatography (24). More recently, Ståhlberg described zone migration of analytes in electrochromatography using mass balance equations ${ }^{(25)}$. Hjertén et al. were the first to point out that the initial stages of the derivation of the mass balance equation make no assumption as to the separation technique. Therefore the equation is generally applicable in separation science(26-28).

The mass balance equation describes the movement of a boundary, where the analyte concentration is different on each side of the boundary, through a tube (see Figure 1.1.1). This can be an open tube, as in free zone electrophoresis, or a packed column, as in chromatography. The concentrations of the analyte $(j)$ on the left and right side of the boundary are $c_{j}^{\alpha}$ and $c_{j}^{\beta}$, respectively. Similarly, the linear velocities of $j$ on the left and right side of the boundary are $v_{j}^{\alpha}$ and $v_{j}^{\beta}$, respectively. Over a period of time $(t)$ the boundary moves from position $A$ to position $B$ at a velocity $v^{\alpha \beta}$. No assumption as to the force (e.g. hydrostatic, centrifugal, electric, etc.) that gives rise to the movement is made. 

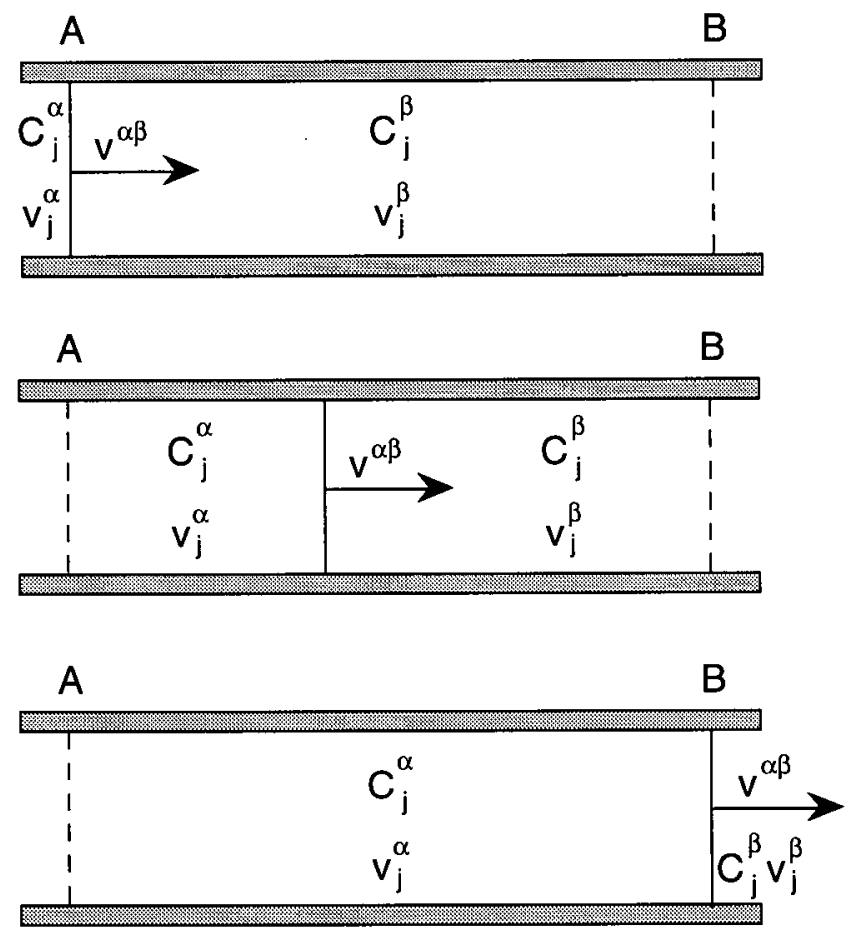

Figure 1.1.1 Representation of the approach used to derive the mass balance equation. Over a period of time $(t)$, at velocity $v^{\alpha \beta}$, the boundary moves from $A$ to $B$ through the volume $V^{\alpha \beta}$. The symbols $c_{j}^{\alpha}, c_{j}^{\beta}, v_{j}^{\alpha}$ and $v_{j}^{\beta}$ represent the concentrations and velocities of the analyte on the left and right sides of the boundary, respectively.

The volume of solution that the boundary moves through (i.e. between A and B) is defined as $V^{\alpha \beta}$. The amount of $j$ in $V^{\alpha \beta}$ before the boundary moves is $c_{j}^{\beta} V^{\alpha \beta}$. Similarly, the amount of $j$ in $V^{\alpha \beta}$ after the boundary moves is $c_{j}^{\alpha} V^{\alpha \beta}$. Therefore, the change in the amount of $j$ in $\mathrm{V}^{\alpha \beta}$ when the boundary moves is:

$$
\Delta \mathrm{j}=\mathrm{V}^{\alpha \beta}\left(\mathrm{c}_{\mathrm{j}}^{\alpha}-\mathrm{c}_{\mathrm{j}}^{\beta}\right)
$$

The change in the amount of $\mathrm{j}$ in $\mathrm{V}^{\alpha \beta}$ can also be determined from the flux of $\mathrm{j}$ across the points $A$ and $B$. The flux of $j$ across point $A$ is given by:

$$
\frac{\Delta \mathrm{j}}{\Delta \mathrm{t}}=\mathrm{c}_{\mathrm{j}}^{\alpha} \mathrm{v}_{\mathrm{j}}^{\alpha} A
$$


where $A$ is the cross sectional area of the tube. Similarly, the flux of $\mathrm{j}$ across point $\mathrm{B}$ is:

$$
\frac{\Delta \mathrm{j}}{\Delta \mathrm{t}}=\mathrm{c}_{\mathrm{j}}^{\beta} \mathrm{v}_{\mathrm{j}}^{\beta} A
$$

The change in the amount of $\mathrm{j}$ in $\mathrm{V}^{\alpha \beta}$ is the difference between eqs 1.1 .2 and 1.1 .3 multiplied by the time required for the boundary to move to position B:

$$
\Delta \mathrm{j}=\mathrm{t} A\left(\mathrm{c}_{\mathrm{j}}^{\alpha} \mathrm{v}_{\mathrm{j}}^{\alpha}-\mathrm{c}_{\mathrm{j}}^{\beta} \mathrm{v}_{\mathrm{j}}^{\beta}\right)
$$

Combining eqs 1.1 .1 and 1.1 .4 gives:

$$
t A\left(c_{j}^{\alpha} v_{j}^{\alpha}-c_{j}^{\beta} v_{j}^{\beta}\right)=V^{\alpha \beta}\left(c_{j}^{\alpha}-c_{j}^{\beta}\right)
$$

Dividing both sides by $\mathrm{t} A$ gives:

$$
c_{j}^{\alpha} v_{j}^{\alpha}-c_{j}^{\beta} v_{j}^{\beta}=v^{\alpha \beta}\left(c_{j}^{\alpha}-c_{j}^{\beta}\right)
$$

where $v^{\alpha \beta}$ is the linear velocity of the boundary.

Most separation methods deal with a narrow band of analyte moving through the tube, not a boundary. The mass balance equation can be applied to these methods if the peak is considered as two moving boundaries. The analyte concentration will often be zero on one side of the boundary, reducing eq 1.1 .6 to:

$$
v_{j}^{\alpha}=v^{\alpha \beta}
$$

Therefore, if the analyte concentration is zero on one side of the boundary, the velocity of the boundary (peak) will be equal to that of the analyte.

Hjertén stated that the existence of an equation generally applicable in separation science suggests that there should be equivalent techniques in chromatography and electrophoresis ${ }^{(26-28)}$. Therefore a phenomenon observable in one technique should be observable in others as well. 


\subsubsection{Virtual Migration Distances}

Rathore and Horváth have compared chromatography and electrophoresis using the concept of virtual migration distances ${ }^{(29)}$. Emphasis was placed on deriving a set of equations for electrophoresis that take a form similar to those used in chromatography. The equations are based on the premise that there are separative and non-separative components to the analyte migration behavior in both techniques. In chromatography, all analytes will have spent an equal amount of time in the mobile phase once they have reached the detector. Therefore, this time is considered non-separative. Conversely, the time the analyte spends in the stationary phase is considered separative. Because the separative and non-separative processes occur in series, their times are additive. In electrophoresis, Rathore and Horváth suggest that the electroosmotic mobility is a non-separative process because all analytes experience the electroosmotic flow (EOF) equally. Electrophoretic mobility is the separative process in electrophoresis. Because the separative and non-separative processes occur in parallel, it is in the velocity (i.e. mobility) domain that the additive relationship occurs.

In order to express the additive relationship for chromatography and electrophoresis in a similar manner, the concept of virtual migration distances was introduced. Virtual migration distances are obtained for both the separative and nonseparative processes. The virtual migration distance represents the distance the analyte would have traveled during the migration time if only one process acted on the analyte. The sum of the separative and non-separative virtual migration distances always equals the actual migration distance.

Rathore and Horváth also define the chromatographic retention factor $\left(k^{\prime}\right)$ and the electrophoretic velocity factor $\left(k_{\mathrm{e}}\right.$ ) in terms of the virtual migration distances ${ }^{(29)}$. The retention factor is written as:

$$
k^{\prime}=\frac{n_{\mathrm{s}}}{n_{\mathrm{m}}}=\frac{\mathrm{t}_{\mathrm{R}}-\mathrm{t}_{0}}{\mathrm{t}_{0}}=\frac{l_{\mathrm{s}}}{l_{0}}
$$


where $n_{\mathrm{s}}$ and $n_{\mathrm{m}}$ are the number of moles of analyte in the stationary and mobile phases respectively, $\mathrm{t}_{\mathrm{R}}$ is the retention time, $\mathrm{t}_{0}$ is the elution time of an unretained marker, and $l_{\mathrm{s}}$ and $l_{0}$ are the separative and non-separative virtual migration distances, respectively. By analogy $k_{\mathrm{e}}^{\prime}$ is defined as:

$k_{\mathrm{e}}^{\prime}=\frac{l_{\mathrm{s}}}{l_{0}}=\frac{\mu_{\mathrm{ep}}}{\mu_{\mathrm{eo}}}=\frac{\mathrm{t}_{\mathrm{eo}}-\mathrm{t}_{\mathrm{R}}}{\mathrm{t}_{\mathrm{R}}}$

where $\mu_{\mathrm{ep}}$ is the electrophoretic mobility, $\mu_{\mathrm{e} o}$ is the electroosmotic mobility, $\mathrm{t}_{\mathrm{eo}}$ is the migration time of an electroosmotic marker and $t_{R}$ is the migration time of the analyte.

While this approach allows the derivation of similar equations in chromatography and electrophoresis, there are some points to consider. In chromatography, $k^{\prime}$ is a thermodynamic constant that describes the phase distribution of the analyte. The electrophoretic velocity factor can make no similar fundamental claim. Instead, $k_{\mathrm{e}}{ }^{\prime}$ compares the electrophoretic mobility of the analyte to the electroosmotic mobility. Since the electroosmotic mobility is notoriously variable, even under similar conditions, it is difficult to see what significance can be derived from $k_{\mathrm{e}}{ }^{\prime}$. It appears that much effort is spent attempting to resolve the separative and non-separative components of chromatography and electrophoresis. Unfortunately, this is not straightforward. It is difficult to conceive how time spent in the stationary phase is separative and in the mobile phase non-separative when it is the equilibrium formed between the two phases that gives rise to separation. Similarly, in electrophoresis the electroosmotic mobility is considered nonseparative even though it has been shown that the EOF can have a major effect on resolution ${ }^{(30-}$ 32). One last point that is not made clear is why all separative or non-separative aspects should be considered analogous, even when vastly different mechanisms are behind the separation. Equilibria (chromatography) and fields (electrophoresis and ultracentrifugation) are fundamentally different, and should therefore be treated differently. Grouping them together gives rise to problems when the virtual migration distances are applied to hybrid techniques such 
as electrochromatography. The EOF is considered non-separative in electrophoresis but would be separative if it gave rise to a bulk flow in chromatography. Therefore, EOF is considered both separative and non-separative in electrochromatography. Although the introduction of virtual migration distances proposed by Rathore and Horváth present similar equations for chromatography and electrophoresis, one must keep in mind that the similar terms in those equations have quite different meanings.

\subsubsection{Conclusions}

Although general theories have previously been developed in separation science, their practical use is limited. The moving boundary technique proposed by Hjertén is generally applicable but does not provide much insight into separation science. The theories developed by Rathore and Horváth give similar expressions in chromatography and electrophoresis. Unfortunately these expressions have quite different meanings. To truly unify separation science, a theory must be developed that describes analyte migration behavior in chromatography, electrophoresis, and ultracentrifugation as well as any hybrid techniques in a manner that provides practical insights into these methods. 


\subsection{A Unified Theory for Separation Science}

\subsubsection{Introduction}

Conventional chromatography theory cannot be used to describe other areas of separation science. This is not surprising, since it was never intended to do so. Conventional chromatography theory is limited by the assumption that all analytes in the mobile phase have the same average linear velocity. Another assumption is that the phase distribution between the mobile and stationary phases is the only equilibrium involving the analyte. These assumptions make chromatography theory inadequate for describing modern separation techniques, which commonly make use of fields or complicated equilibria. The following section describes a general separation theory that does not make these assumptions. This allows a better understanding of the individual separation techniques, especially hybrid techniques, such as MECC and electrochromatography, which are not easily described using conventional theories.

\subsubsection{A General Equation for Separation Science}

Based on the definition for the speciation of an element ${ }^{(33)}$, the species of an analyte can be defined as the individual physicochemical forms of the analyte that together, through various types of equilibria, constitute its total amount in a system. The individual physicochemical forms include gaseous, liquid, solid compounds, dissolved forms in different immiscible solvents, as well as ionic and non-covalent complexes formed with different reagents. An analyte may be distributed among different species due to partition between phases, dynamic complexation processes, acid-base equilibria, etc. For example, in a common chromatographic system there are two analyte species (the analyte in the mobile phase and the analyte in the stationary phase) because the analyte is distributed between two immiscible phases. In CE, different analyte species are often present (in the same phase) due to dynamic complexation between analytes and additives. The migration rate of an analyte is determined by the intrinsic migration rates for each species and the fraction of analyte present as each species: 
$\overline{\mathrm{U}}^{\mathrm{A}}=\sum_{\mathrm{i}=1}^{\mathrm{m}} f_{\mathrm{i}} \overline{\mathrm{U}}_{\mathrm{i}}$

where $\overline{\mathrm{U}}^{\mathrm{A}}$ is the average migration rate of the analyte, $\mathrm{m}$ is the number of analyte species (including the free analyte), $f_{\mathrm{i}}$ is the fraction of analyte present as species $\mathrm{i}$, and $\overline{\mathrm{U}}_{\mathrm{i}}$ is the migration rate of species $i$. It should be emphasized that this equation is generally true for all types of equilibria in all areas of separation science. The only assumption made is that the equilibria between species take place at a much faster rate than the overall separation process.

Analytes can be separated using equilibria if the distribution of a pair of analytes between species is different (i.e. $f_{\mathrm{i}}$ are different for each analyte). This is the case in chromatography where differential migration of analytes occurs due to the differential distribution of analytes between the stationary and mobile phases. If the analytes respond to a physical field differently, separation can be achieved through the use of fields. This is the case in ultracentrifugation and carrier free CE where although there is only one species (i.e. the free analyte) for each analyte, separation can be achieved if the analytes migrate at different velocities in the field. When additives are used in CE, both equilibria and field determine the analyte migration rate.

\subsubsection{Individual Capacity Factors}

The capacity factor $\left(k^{\prime}\right)$ is the most important parameter in describing the effect of equilibria on a separation. In order to unify separation science, it is necessary to use a general definition for $k^{\prime}$ which can be applied to any equilibrium that may be encountered.

In chromatography, the primary definition of $k^{\prime}$ is ${ }^{(34)}$ :

$k^{\prime}=\frac{\text { the amount of analyte in the stationary phase }}{\text { the amount of analyte in the mobile phase }}$

This definition is sufficient to describe chromatographic systems where there are two well- 
defined analyte species. A more general equation is necessary to describe the more complicated equilibria that are commonly encountered in CE. Peng et al. ${ }^{(35,36)}$ have proposed the following definition of $k^{\prime}$ for $\mathrm{CE}$ where complexation takes place with one or more additives:

$k^{\prime}=\frac{\text { the amount of analyte present as a specific complex }}{\text { the amount of free analyte }}$

For a unified separation science, the definition of $k^{\prime}$ can be generalized to include any equilibrium:

$k_{\mathrm{i}}^{\prime}=\frac{\text { the amount of analyte species } \mathrm{i}}{\text { the amount of free analyte }}$

Using this definition, there is a capacity factor for each analyte species. The capacity factor for the free analyte (i.e. when species $i$ in eq 1.2 .4 is the free analyte) is equal to one. In more complicated systems, where several equilibria take place, it may become unclear which species of the analyte should be referred to as the "free analyte". In most cases the free analyte can be defined as the uncomplexed analyte species in the mobile phase (if applicable).

While the definition of $k^{\prime}$ given in eq 1.2.4 remains the same for all types of equilibria, the way in which it is calculated depends on how the equilibrium is described. In chromatography, where the analyte partitions between two phases, the equilibrium constant $(\mathrm{K})$ is defined as:

$\mathrm{K}=\frac{[\mathrm{A}]_{\mathrm{S}}}{[\mathrm{A}]_{\mathrm{M}}}$

where $[\mathrm{A}]_{\mathrm{S}}$ and $[\mathrm{A}]_{\mathrm{M}}$ are the concentrations of analyte in the stationary and mobile phases, respectively. Based on this definition of $\mathrm{K}$, the capacity factor for the analyte in the stationary phase $\left(k_{\mathrm{A}, \mathrm{S}}^{\prime}\right)$ can be expressed as:

$k_{\mathrm{A}, \mathrm{S}}^{\prime}=\frac{\text { the amount of analyte in the stationary phase }}{\text { the amount of free analyte }}=\mathrm{K}\left(\frac{\mathrm{V}_{\mathrm{S}}}{\mathrm{V}_{\mathrm{M}}}\right)$ 
where $V_{S}$ and $V_{M}$ are the volumes of the stationary and mobile phases respectively (the corrected volume, $\mathrm{V}_{\mathrm{M}}^{0}$, is used in GC).

In CE, most equilibria involve dynamic complexation (with a 1:1 stoichiometry) between the analyte and a buffer additive. In this case the equilibrium constant is defined as:

$$
\mathrm{K}=\frac{[\mathrm{AC}]}{[\mathrm{A}][\mathrm{C}]} \cdot \cdot
$$

where $[\mathrm{AC}]$ is the concentration of the analyte-additive complex, $[\mathrm{A}]$ is the concentration of the analyte, and $[\mathrm{C}]$ is the concentration of the additive. Because the complexation takes place in a single phase, the capacity factor for the analyte in the complexed form $\left(k_{\mathrm{AC}}^{\prime}\right)$ is:

$$
k_{\mathrm{AC}}^{\prime}=\frac{\text { the amount of complex AC }}{\text { the amount of free analyte }}=\frac{[\mathrm{AC}]}{[\mathrm{A}]}=\mathrm{K}[\mathrm{C}]
$$

The capacity factors for complexes with different stoichiometries will be discussed in chapter 1.3 .

It should be emphasized that the definition for the capacity factor given in eq 1.2.4 is the same for both chromatography and CE. The capacity factors are calculated differently because different equilibria are used to describe the methods. Although the expressions for $k^{\prime}$ change, the primary definition of the capacity factor (eq. 1.2.4), and therefore the actual value of the capacity factor, is not affected by the way in which the equilibrium is described. MECC can be used to illustrate this point. Early models describing MECC treated micelles as a pseudo-stationary phase. The equilibrium constant and capacity factor were described based on the partition of the analyte between phases using equations similar to eqs 1.2.5 and 1.2.6. Alternatively, micelles can be treated, not as a separate phase, but as a discrete complexation additive which undergoes dynamic complexation with the analyte ${ }^{(35)}$. The equilibrium constant and capacity factor can now be described using eqs 1.2 .7 and 1.2.8. The two methods yield very different values for $\mathrm{K}$ because they are based on different equilibria. The volumes used to calculate the analyte 
concentration in the pseudo-stationary phase and the complex concentration in a homogeneous solution are also very different. Although the values of $\mathrm{K}$ will be different, both methods should give the same value for the capacity factor because the definition for $k^{\prime}$ is identical. In situations where more than one model can be used to describe a particular equilibrium, the methods for calculating $k^{\prime}$ should be compared to determine which is more practical.

The fraction of analyte present as each species can be calculated according to:

$$
f_{\mathrm{i}}=\frac{k_{\mathrm{i}}^{\prime}}{\sum_{\mathrm{i}=1}^{\mathrm{m}} k_{\mathrm{i}}^{\prime}}
$$

Equation 1.2.9 can be substituted into eq 1.2.1 to give:

$$
\overline{\mathrm{U}}^{\mathrm{A}}=\frac{\sum_{\mathrm{i}=1}^{\mathrm{m}} k_{\mathrm{i}}^{\prime} \overline{\mathrm{U}}_{\mathrm{i}}}{\sum_{\mathrm{i}=1}^{\mathrm{m}} k_{\mathrm{i}}^{\prime}}
$$

Eq 1.2.10 is a general equation that can be used to describe the migration behavior of any analyte in any separation technique without limitation as to the number or type of equilibria. The individual capacity factors, in the presence of more than one analyte species, indicate the tendency of the analyte to exist as a particular species in a competitive environment. An increase in the capacity factor may or may not lead to an increased fraction of a certain species because the fraction of analyte present as a particular species also depends on the capacity factors of the other species. Using individual capacity factors for the description of analyte migration behavior allows a better understanding of the chemical separation process, which allows the systematically design of separation systems according to the physicochemical properties of the analyte, the various immiscible phases, and the various types of additives. 


\subsubsection{The Effect of Field}

Field is the other governing factor that contributes to separation processes. Fields can be employed in chemical separations when the migration rates of corresponding species for a pair of analytes are different. This is common in electrophoresis and centrifugation where the migration rate of an analyte species depends on its intrinsic properties.

In electrophoresis and centrifugation, it is common to use mobilities (migration rate divided by the magnitude of the field) to describe the analyte migration behavior because mobility is independent of the field strength. During a separation, all species of an analyte experience the same field, allowing eq 1.2.10 to be expressed in terms of mobility:

$$
\mu^{\mathrm{A}}=\frac{\sum_{\mathrm{i}=1}^{\mathrm{m}} k_{\mathrm{i}}^{\prime} \mu_{\mathrm{i}}}{\sum_{\mathrm{i}=1}^{\mathrm{m}} k_{\mathrm{i}}^{\prime}}
$$

where $\mu_{\mathrm{i}}$ is the mobility of analyte species $\mathrm{i}$, determined by the intrinsic properties of the analyte.

In CE, the electrophoretic mobility of an analyte $\left(\mu_{\mathrm{ep}, \mathrm{A}}\right)$ is given by:

$$
\mu_{\mathrm{ep}, \mathrm{A}}=\frac{\mathrm{Q}_{\mathrm{eff}, \mathrm{A}}}{6 \pi \eta \mathrm{R}_{\mathrm{A}}}
$$

where $Q_{\text {eff, } A}$ is the effective charge of the analyte, $\eta$ is the viscosity of the background electrolyte, and $R_{A}$ is the radius of the analyte. The sedimentation coefficient $\left(s_{A}\right)$ is the parameter equivalent to mobility in ultracentrifugation:

$$
s_{\mathrm{A}}=\frac{\Phi\left(\rho_{\mathrm{A}}-\rho_{\mathrm{m}}\right)}{6 \pi \eta \mathrm{R}_{\mathrm{A}}}
$$

where $\Phi$ is the volume of the analyte, $\rho_{A}$ is the density of the analyte, and $\rho_{m}$ is the density of the medium. The migration rate of an analyte in a centrifugal field is equal to its sedimentation coefficient times the centrifugal force (which is equal to $\omega^{2} \mathrm{r}$, where $\omega$ is the angular velocity of 
the rotor, and $\mathrm{r}$ is the radial distance from the axis of rotation).

Equations 1.2.12 and 1.2.13 clearly show that the migration rate of an analyte species in an electric or centrifugal field depends on the intrinsic properties of the species, thus allowing separation even in the absence of equilibria. This is the case in ultracentrifugation and carrier free CE where there is only one analyte species (i.e. the free analyte), and therefore no equilibrium.

Chromatography presents a special case due to the absence of the field effect. The migration rates of the individual analyte species do not depend on the physical properties of the species. Instead, the species can be divided into two groups: those in the stationary phase have a migration rate of zero; those in the mobile phase have a migration rate equal to the velocity of the mobile phase. Equation 1.2.10 can therefore be simplified to:

$\overline{\mathrm{U}}^{\mathrm{A}}=\frac{\sum \text { capacity factors of species in the mobile phase }}{\sum \text { capacity factors of all species }} \overline{\mathrm{U}}_{0}$

where $\overline{\mathrm{U}}_{0}$ is the average linear velocity of the mobile phase. In a simple chromatography system where there is only one species in the mobile phase (the free analyte) and one in the stationary phase, eq 1.2.14 simplifies to:

$\overline{\mathrm{U}}^{\mathrm{A}}=\frac{1}{1+k_{\mathrm{A}, \mathrm{S}}^{\prime}} \overline{\mathrm{U}}_{0}$

which is the equation most often used in chromatography. The " 1 " in eq 1.2.15 is the capacity factor of the free analyte in the mobile phase. The advantage of eq 1.2 .14 is its ability to describe the increasingly complex equilibria, such as acid-base equilibria or ion-pair interactions, that are becoming common in chromatography. Additives such as cyclodextrins can also be used in the mobile phase to introduce additional equilibria ${ }^{(37)}$. There is also the possibility of multiple species in the stationary phase. If a column is packed with more than one stationary phase, the 
migration behavior of an analyte can be predicted if the capacity factor for the analyte in each stationary phase is known. Equation 1.2.14 allows a better understanding of how multiple interactions affect the migration behavior of analytes in complex chromatographic separation systems.

Because the migration rate of an analyte in $\mathrm{CE}$ with additives is determined by both equilibria and field, dynamic complexation $\mathrm{CE}$ is one of the most versatile separation techniques available. It combines the separation power of both chromatography (using equilibrium) and carrier free CE (using field).

With the capacity factor and the effect of field in perspective, the relationship between the three major areas of separation science can be clearly observed. Each area can be classified as one aspect of a general separation science. The parameters and fundamental equations in CE, chromatography, and ultracentrifugation are compared in Table 1.2.1. 
16.

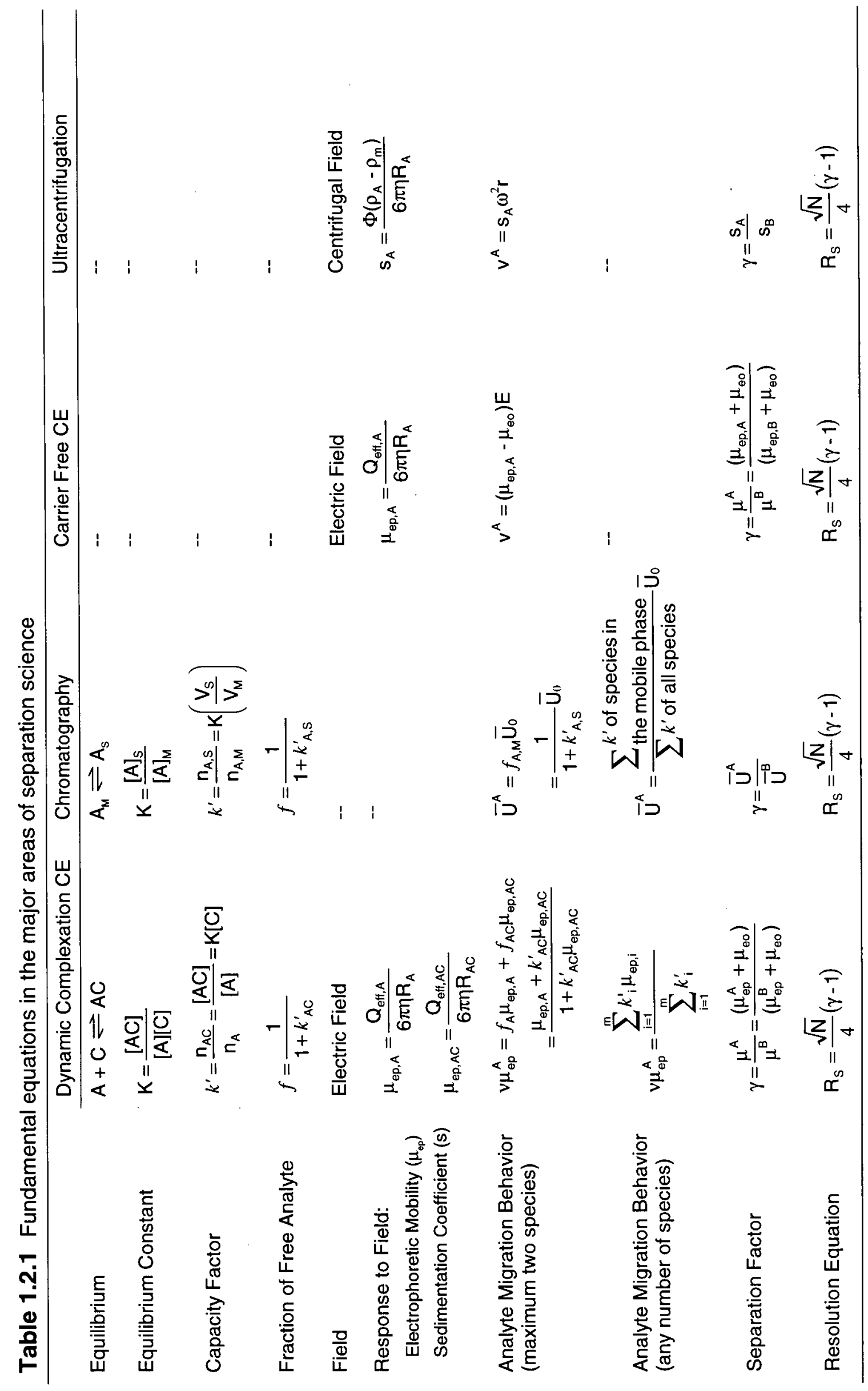




\subsubsection{The Separation Factor}

In chromatography, the resolution equation has been divided into three components: the column efficiency (related to the number of theoretical plates, N), the selectivity $(\alpha)$, and the retention (related to $k$ ):

$$
\mathrm{R}_{\mathrm{s}}=\left(\frac{\sqrt{\mathrm{N}}}{4}\right)\left(\frac{\alpha-1}{\alpha}\right)\left(\frac{k_{\mathrm{B}}^{\prime}}{1+k_{\mathrm{B}}^{\prime}}\right)
$$

The selectivity is equal to the ratio of the capacity factors of the two analytes $\left(k_{\mathrm{B}}^{\prime} / k_{\mathrm{A}}^{\prime}\right)$. This ratio is derived from the primary definition of $\alpha^{(34) \text { : }}$

$$
\alpha=\frac{C_{\mathrm{A}}^{\mathrm{M}} / \mathrm{C}_{\mathrm{A}}^{\mathrm{S}}}{\mathrm{C}_{\mathrm{B}}^{\mathrm{M}} / \mathrm{C}_{\mathrm{B}}^{\mathrm{S}}}
$$

where $C_{A}^{M}, C_{A}^{S}, C_{B}^{M}$, and $C_{B}^{S}$ are the concentrations of analytes $A$ and $B$ in the two phases (mobile and stationary), respectively. The concept of selectivity was devised to compare the affinity of two analytes to a particular stationary phase in relation to the mobile phase. While this is useful in comparing stationary phases, the relationship between $\alpha$ and the resolution of a pair of analytes is not simple. As can be seen in eq 1.2.16, the selectivity and retention terms in the resolution equation are not independent. Because $\alpha$ is defined as $\mathrm{k}_{\mathrm{B}}^{\prime} / \mathrm{k}_{\mathrm{A}}^{\prime}$, when the selectivity is changed, the retention term is also affected, and vice versa. This point is illustrated in Figure 1.2.1 by plotting resolution vs. $k_{\mathrm{B}}^{\prime}$. 


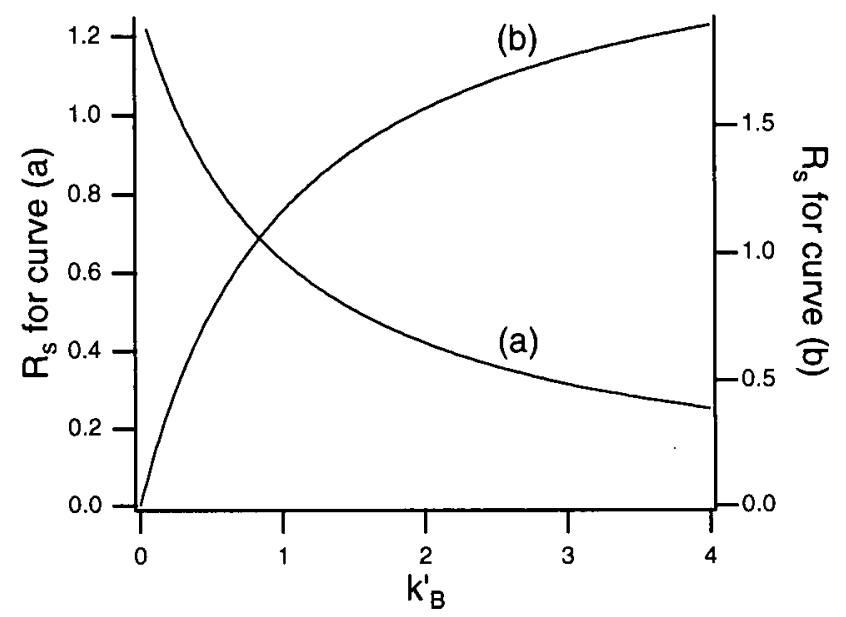

Figure 1.2.1 Effect of $k_{\mathrm{B}}^{\prime}$ on Resolution. The difference between $k_{\mathrm{B}}^{\prime}$ and $k_{\mathrm{A}}^{\prime}$ is constant in curve (a) $\left(\mathrm{N}=10,000 ;\left(k_{\mathrm{B}}^{\prime}-k_{\mathrm{A}}^{\prime}\right)=0.05 ; k_{\mathrm{B}}^{\prime}=0.05\right.$ to $4 ; \bar{U}_{0}=15 \mathrm{~cm} / \mathrm{min}$; and column length $=15 \mathrm{~cm}$ ). The ratio of $k_{\mathrm{B}}^{\prime}$ and $k_{\mathrm{A}}^{\prime}$ is constant in curve (b) $\left(\mathrm{N}=10,000 ; \mathrm{K}_{\mathrm{A}}=10 ; \mathrm{K}_{\mathrm{B}}=11 ; \overline{\mathrm{U}}_{0}=15 \mathrm{~cm} / \mathrm{min}\right.$.; column length $=15 \mathrm{~cm}$; and $V_{S} N_{M}=0$ to 0.4$)$.

Curve (a) in Figure 1.2.1 shows that when the difference between the two capacity factors remains the same, increasing the retention time of analyte $\mathrm{B}$ actually decreases the resolution. Conversely, if the ratio of the two capacity factors is kept the same, as demonstrated by curve (b) in Figure 1.2.1, the resolution does increase with a longer retention time.

While the standard resolution equation (eq 1.2.16) has proven its usefulness in describing simple chromatographic systems, it is not sufficient to be used generally in a unified separation science because one important aspect of separation science (i.e. field) was not considered in deriving this equation. Because eq 1.2.16 does not include the effects of field, it can not be used in ultracentrifugation and carrier free $\mathrm{CE}$ where field governs the migration behavior of the analytes. The definition of $\alpha$ also prevents eq 1.2.16 from being extended to situations where the analyte is distributed between more than two species. The selectivity only compares the affinity of a pair of analytes to a single additive or phase and cannot be used to predict the distribution of the analyte in cases where there are more than two analyte species. More recently researchers have attempted to model chromatographic separations based on the kinetics of the adsorption process, thereby allowing a better understanding of the effect certain properties of the stationary 
phase have on analyte migration behavior ${ }^{(38-40)}$. Unfortunately this approach has not yet been applied to cases where the analyte is involved in complicated equilibria.

To derive a generally applicable resolution equation for a unified separation science, we need to define a new separation factor, $\gamma$ :

$\gamma=\frac{\overline{\mathrm{U}}^{\mathrm{A}}}{\overline{\mathrm{U}}^{\mathrm{B}}}$

where $\overline{\mathrm{U}}^{\mathrm{A}}$ and $\overline{\mathrm{U}}^{\mathrm{B}}$ are the migration rates of the two analytes. The value of $\gamma$ describes the relative migration behavior, including the effects of both equilibrium and field, of a pair of analytes, and therefore is more directly related to the resolution than $\alpha$. Equation 1.2.18 assumes that both analytes migrate in the same direction, and $\bar{U}_{A}$ is greater than $\bar{U}_{B}$. Therefore, $\gamma$ is always greater than or equal to 1 . If the analytes are moving in different directions, it is not necessary to use the separation factor since the resolution will approach infinity.

In carrier free $\mathrm{CE}$, the ratio of the mobilities can be substituted for the ratio of migration rates since the electric field is the same for all analytes. Therefore, $\gamma$ can be written as:

$\gamma=\frac{\mu^{\mathrm{A}}}{\mu^{\mathrm{B}}}=\frac{\mu_{\mathrm{ep}, \mathrm{A}}+\mu_{\mathrm{eo}}}{\mu_{\mathrm{ep}, \mathrm{B}}+\mu_{\mathrm{eo}}}$

Where $\mu_{\mathrm{e} o}$ is the electroosmotic mobility which is a result of the electroosmotic flow (EOF) in a capillary. The same concept can be applied to ultracentrifugation where the migration rate is determined by the centrifugal field. The migration rates in eq 1.2 .18 are replaced by the sedimentation coefficients, as shown in the last column of Table 1.2.1.

When additives are used in $\mathrm{CE}$, the analyte migration behavior is dictated by the dynamic complexation process between the analyte and the additives as well as the electric field. Both equilibria and the electrophoretic mobilities govern the separation. The separation factor should be defined as the ratio of the net mobilities: 
$\gamma=\frac{\mu^{\mathrm{A}}}{\mu^{\mathrm{B}}}=\frac{\mu_{\mathrm{ep}}^{\mathrm{A}}+\mu_{\mathrm{eo}}}{\mu_{\mathrm{ep}}^{\mathrm{B}}+\mu_{\mathrm{eo}}}$

Although this definition looks similar to the definition for carrier free CE, $\mu_{\mathrm{ep}}^{\mathrm{A}}$ and $\mu_{\mathrm{ep}}^{\mathrm{B}}$ include the effects of equilibrium, in addition to those of field.

The separation factor, $\gamma$, can be simplified in chromatography because there is no field effect. Since the migration rate of all analytes in the mobile phase is equal to the average linear flow rate of the mobile phase, $\gamma$ is equal to the ratio of the fractions of the analytes in the mobile phase:

$\gamma=\frac{f_{\mathrm{A}}^{\mathrm{M}}}{f_{\mathrm{B}}^{\mathrm{M}}}=\frac{\left(\frac{\sum k_{\mathrm{A}}^{\prime} \text { of species in the mobile phase }}{\sum k_{\mathrm{A}}^{\prime} \text { of all species }}\right)}{\left(\frac{\sum k_{\mathrm{B}}^{\prime} \text { of species in the mobile phase }}{\sum k_{\mathrm{B}}^{\prime} \text { of all species }}\right)}$

where $f_{\mathrm{A}}^{\mathrm{M}}$ and $f_{\mathrm{B}}^{\mathrm{M}}$ are the fractions of analytes $\mathrm{A}$ and $\mathrm{B}$ in the mobile phase.

Defining the separation factor according to eq 1.2.18 allows the resolution equation to be generally written as:

$\mathrm{R}_{\mathrm{S}}=\frac{\mathrm{t}_{\mathrm{R}}^{\mathrm{B}}-\mathrm{t}_{\mathrm{R}}^{\mathrm{A}}}{\frac{1}{2}\left(\mathrm{~W}_{\mathrm{A}}+\mathrm{W}_{\mathrm{B}}\right)}=\frac{\sqrt{\mathrm{N}}}{4}(\gamma-1)$

where $t_{R}^{A}, t_{R}^{B}$, and $W_{A}, W_{B}$ are the migration times and peak widths of the analytes $A$ and $B$, respectively. Equation 1.2.22 shows that resolution is determined by the column efficiency and $\gamma$ for all separation techniques. More importantly, because $\gamma$ is defined as the ratio of the migration rates, which accounts for all of the possible analyte species in a separation system, the resolution can be predicted regardless of the number or the type of the equilibria involved. The separation factor, $\gamma$, also accounts for species with different mobilities in the electric (or a centrifugal) field. 
The presence of the EOF is a unique phenomenon in CE related separation techniques. The EOF is a bulk property experienced by all analyte species, superimposed on the individual mobilities of each species. Equations 1.2.19 and 1.2.20 have included the effect of EOF. If the EOF and electrophoretic mobilities of the analyte pair are in opposite directions, resolution approaches infinity as the magnitude of $\mu_{\mathrm{e} o}$ approaches the electrophoretic mobilities. The denominator approaches zero before the numerator because according to the definition of $\gamma, \overline{\mathrm{U}}^{\mathrm{A}}$ is greater than $\bar{U}^{B}$. The effect of the EOF on resolution is demonstrated in Figure 1.2.2. Similar trends have been observed previously ${ }^{(30,41,42)}$. The effect of the EOF on resolution will be discussed further in chapters 1.5 and 3.2. If the EOF is in the same direction as the electrophoretic mobilities of the analytes, any increase in $\mu_{\mathrm{e} 0}$ decreases $\gamma$, and therefore the resolution.

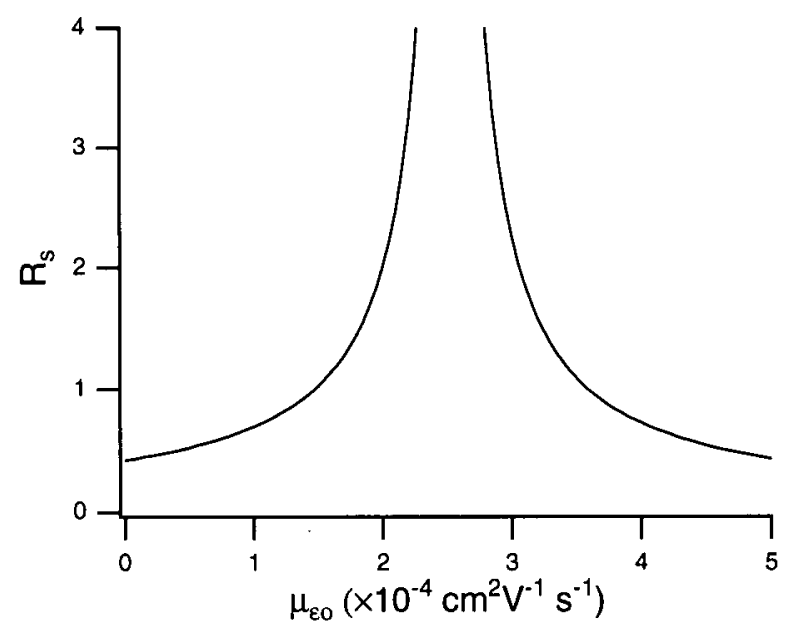

Figure 1.2.2 The effect of the EOF on resolution. The values used to generate the curve are: $N=20,000 ; \mu_{\mathrm{ep}}^{\mathrm{A}}=-2.5 \times 10^{-4} \mathrm{~cm}^{2} \mathrm{~V}^{-1} \mathrm{~s}^{-1}$; $\mu_{\mathrm{ep}}^{\mathrm{B}}=-2.53 \times 10^{-4} \mathrm{~cm}^{2} \mathrm{~V}^{-1} \mathrm{~s}^{-1} ; \mu_{\mathrm{oo}}=0$ to $0.005 \mathrm{~cm}^{2} \mathrm{~V}^{-1} \mathrm{~s}^{-1}$; capillary length $=$ $57 \mathrm{~cm}$; length to detector $=50 \mathrm{~cm}$; voltage $=30 \mathrm{kV}$.

\subsubsection{Conclusions}

Separation theory is greatly simplified by clarifying the meaning of the capacity factor and redefining the separation factor. All areas of separation science can be described according 
to a general theory (most importantly, eqs $1.2 .1,1.2 .10$, and 1.2.22). The theory not only describes the separation process of each technique, but is also instrumental to the further development of separation science. For example, the knowledge obtained from the study of multiple additives in CE can be extended to the design of chromatography columns with multiple stationary phases (i.e. different packing materials in one column). Different packing materials could be mixed in calculated proportions to achieve a predicted separation. Dynamic complexation $\mathrm{CE}$ is the most often used hybrid technology to date. Capillary electrochromatography is another newly developed separation technique. A generalized theory with clearly defined interchangeable parameters, such as the separation factor and the capacity factor, is essential in the description of these hybrid separation systems. The unified approach presented here should be useful in the development of new hybrid techniques as well as the further advancement of existing methods. 


\subsection{Higher Order Equilibria and Their Effect on Analyte Migration Behavior in Capillary Electrophoresis}

\subsubsection{Introduction}

The use of complexation additives to modify analyte migration behavior in capillary electrophoresis (CE) has increased dramatically in recent years. Terabe et al. were the first to

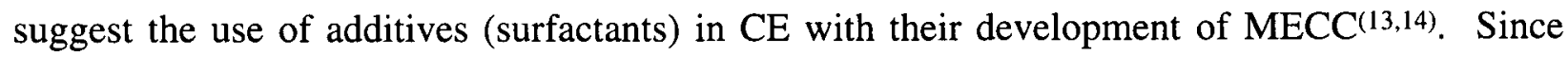
then, applications using additives such as cyclodextrins ${ }^{(43-45)}$, polyethers ${ }^{(31,46,47)}$, and macrocyclic antibiotics ${ }^{(48-50)}$ have been reported.

Recent developments in separation science, considering interactions at the molecular level, have extended CE theory to the point where analyte migration behavior can be described quantitatively. A number of papers discussing analyte-additive interactions in protein binding ${ }^{(51)}$ protein-sugar interactions ${ }^{(52,53)}$, chiral separations ${ }^{(45,54,55)}$, cyclodextrin complexes ${ }^{(35,44,48,56-63)}$, glycopeptide interactions ${ }^{(64)}$, antigen-antibody complexes ${ }^{(65)}$, and nonaqueous separations ${ }^{(47,66,67)}$, have been published. All of these papers describe analyte migration behavior based on a dynamic equilibrium process between an analyte and a single additive with a 1:1 binding stoichiometry.

A common assumption in $\mathrm{CE}$ binding studies to date is that the analyte-additive interaction can be sufficiently described by a 1:1 stoichiometry. While it is true that the majority of interactions encountered in $\mathrm{CE}$, especially when the additive concentration is relatively low or there is only one binding site on the analyte, take place with a 1:1 stoichiometry, there are situations where higher order equilibria (i.e. interactions with stoichiometries other than 1:1) are significant. Determining the correct stoichiometry of an interaction is a crucial step in quantitatively describing the effect of an additive on the migration behavior of the analyte. If the wrong equilibrium model is chosen, the calculated constants will reflect neither the real interaction between the analyte and the additive or their actual effect on the net mobility of the 
analyte. To systematically design a CE separation, these interactions must be understood and the associated constants accurately determined.

With a few exceptions ${ }^{(68-70)}$, papers published to date have only considered equilibria with 1:1 (analyte:additive) stoichiometries between the analyte and a single additive. An equation was recently proposed that describes analyte migration behavior in the presence of any number of 1:1 interactions for CE systems with one or more additives ${ }^{(35)}$. Vogt et al. used equilibrium constants determined by other techniques as a qualitative guide in designing a $\mathrm{CE}$ separation of rare earth metals through higher order complexation with organic ligands ${ }^{(68,70)}$. Mammen et al. demonstrated a CE method describing the interaction of an antibody with two ligands ${ }^{(69)}$. The method can only be applied to interactions with binding constants greater than $10^{6} \mathrm{M}^{-1}$ because the mobility of the fully complexed (1:2) analyte must be measured directly.

The aim of the following section is to describe a generally applicable method for the detection and quantification of higher order interactions in CE. A common 1:1 interaction will be compared with higher order interactions in which two additive molecules can bind with a single analyte. Hydroxypropyl- $\beta$-cyclodextrin $(\mathrm{HP}-\beta-\mathrm{CD})$ is used as the additive for several reasons: 1) it is a rigid host molecule with one cavity in which well defined interactions take place; 2) cyclodextrins are one of the most commonly used buffer additives in CE; 3) HP- $\beta$-CD has a relatively high solubility in aqueous buffers. Phenol is used as an analyte that only interacts with HP- $\beta-C D$ at a 1:1 stoichiometry; 4,4'-biphenol and 4-phenylphenol each have two binding sites that can interact with HP- $\beta-C D$ and are used to demonstrate the effect of higher order equilibria. 


\subsubsection{Theory}

The first step in describing the effect of an additive on the migration behavior of an analyte is to derive the necessary equations. The equation describing a 1:1 analyte:additive interaction has been previously derived(55,58,59,62) and has recently been modified by the introduction of a correction factor ${ }^{(35,66)}$ to allow the equilibrium to be studied independently of other factors which may affect analyte migration behavior. When both 1:1 and 1:2 analyteadditive interactions are present, the equations must be modified to reflect the presence of higher order equilibria.

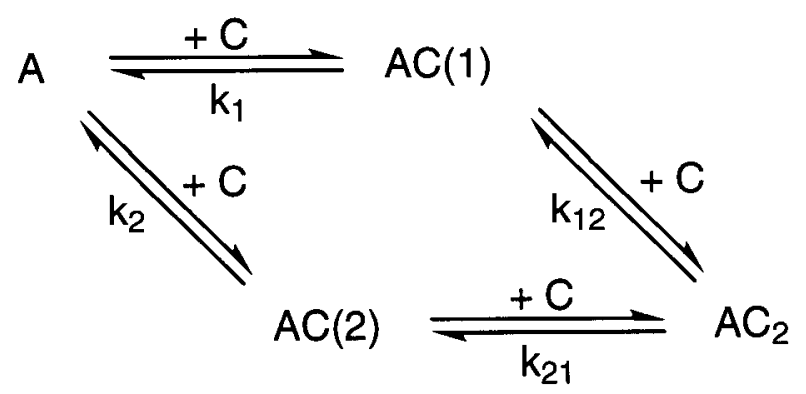

Figure 1.3.1 Equilibria between an analyte (A) with two binding sites and an additive (C) with one binding site. The numbers in parentheses represent the binding site(s) on the analyte that is bound to the additive.

An analyte (A) with two binding sites (site 1 and site 2) can interact with an additive (C) according to the equilibrium shown in Figure 1.3.1. The numbers in parentheses represent the site(s) of interaction and the constants $k_{i j}$ are the microscopic equilibrium constants, where $i$ and $j$ represent the two different binding sites. According to this equilibrium, at any given time a certain fraction of the analyte will be uncomplexed (A), singly complexed at binding site 1 $(\mathrm{AC}(1))$, singly complexed at binding site $2(\mathrm{AC}(2))$, and doubly complexed $\left(\mathrm{AC}_{2}(1,2)\right)$. The net electrophoretic mobility of the analyte $\left(\mu_{\mathrm{ep}}^{\mathrm{A}}\right)$ will be determined by the fraction of the analyte present as each of these species $(f)$ and the electrophoretic mobility of each of these species $\left(\mu_{\mathrm{ep}}\right)$ :

$v \mu_{\mathrm{ep}}^{\mathrm{A}}=f_{\mathrm{A}} \mu_{\mathrm{ep}, \mathrm{A}}+f_{\mathrm{AC}(1)} \mu_{\mathrm{ep}, \mathrm{AC}(1)}+f_{\mathrm{AC}(2)} \mu_{\mathrm{ep}, \mathrm{AC}(2)}+f_{\mathrm{AC}(1,2)} \mu_{\mathrm{ep}, \mathrm{AC} 2(1,2)}$

where $v$ is a correction factor that accounts for changes in viscosity caused by the complexation 
additive $(35,66)$. The fractions of the analyte in each species are given by:

$$
\begin{aligned}
& f_{\mathrm{A}}=\frac{1}{1+\mathrm{k}_{1}[\mathrm{C}]+\mathrm{k}_{2}[\mathrm{C}]+\mathrm{k}_{1} \mathrm{k}_{12}[\mathrm{C}]^{2}} \\
& f_{\mathrm{AC}(1)}=\frac{\mathrm{k}_{1}[\mathrm{C}]}{1+\mathrm{k}_{1}[\mathrm{C}]+\mathrm{k}_{2}[\mathrm{C}]+\mathrm{k}_{1} \mathrm{k}_{12}[\mathrm{C}]^{2}} \\
& f_{\mathrm{AC}(2)}=\frac{\mathrm{k}_{2}[\mathrm{C}]}{1+\mathrm{k}_{1}[\mathrm{C}]+\mathrm{k}_{2}[\mathrm{C}]+\mathrm{k}_{1} \mathrm{k}_{12}[\mathrm{C}]^{2}} \\
& f_{\mathrm{AC}_{2}}=\frac{\mathrm{k}_{1} \mathrm{k}_{12}[\mathrm{C}]^{2}}{1+\mathrm{k}_{1}[\mathrm{C}]+\mathrm{k}_{2}[\mathrm{C}]+\mathrm{k}_{1} \mathrm{k}_{12}[\mathrm{C}]^{2}}=\frac{\mathrm{k}_{2} \mathrm{k}_{21}[\mathrm{C}]^{2}}{1+\mathrm{k}_{1}[\mathrm{C}]+\mathrm{k}_{2}[\mathrm{C}]+\mathrm{k}_{1} \mathrm{k}_{12}[\mathrm{C}]^{2}}
\end{aligned}
$$

Inserting the fractions of each analyte species (eqs 1.3.2-1.3.5) into eq 1.3.1 gives:

$v \mu_{\mathrm{ep}}^{\mathrm{A}}=\frac{\mu_{\mathrm{ep}, \mathrm{A}}+\mathrm{k}_{1} \mu_{\mathrm{ep}, \mathrm{AC}(1)}[\mathrm{C}]+\mathrm{k}_{2} \mu_{\mathrm{ep}, \mathrm{AC}(2)}[\mathrm{C}]+\mathrm{k}_{1} \mathrm{k}_{12} \mu_{\mathrm{ep}, \mathrm{AC}}[\mathrm{C}]^{2}}{1+\mathrm{k}_{1}[\mathrm{C}]+\mathrm{k}_{2}[\mathrm{C}]+\mathrm{k}_{1} \mathrm{k}_{12}[\mathrm{C}]^{2}}$

which can be simplified to:

$$
v \mu_{\mathrm{ep}}^{\mathrm{A}}=\frac{\mu_{\mathrm{ep}, \mathrm{A}}+\left(\mathrm{k}_{1} \mu_{\mathrm{ep}, \mathrm{AC}(1)}+\mathrm{k}_{2} \mu_{\mathrm{ep}, \mathrm{AC}(2)}\right)[\mathrm{C}]+\mathrm{k}_{1} \mathrm{k}_{12} \mu_{\mathrm{ep}, \mathrm{AC}}[\mathrm{C}]^{2}}{1+\left(\mathrm{k}_{1}+\mathrm{k}_{2}\right)[\mathrm{C}]+\mathrm{k}_{1} \mathrm{k}_{12}[\mathrm{C}]^{2}}
$$

Sänger - van de Griend(71) recently used an equation similar to eq 1.3 .7 to qualitatively discuss possible 1:2 interactions between a tetrapeptide and a derivatized $\beta$-CD. Equation 1.3.7 shows that the microscopic equilibrium constants for the first order equilibria can be combined into a single constant (e.g. $\left(k_{1}+k_{2}\right)$ in the denominator). It is generally true that the observed equilibrium constants are the sum of the microscopic equilibrium constants for interactions of the same order with respect to the additive concentration. It is therefore impossible to obtain the individual microscopic constants when measuring the effect of $[\mathrm{C}]$ on the net analyte mobility. The observed equilibrium constants have been referred to as macroscopic ${ }^{(72)}$ or stoichiometric constants ${ }^{(73)}$ since all interactions with the same stoichiometry are grouped together. Because the 
macroscopic constants do not discriminate between complexes with the same binding stoichiometry (e.g. $\mathrm{AC}(1)$ and $\mathrm{AC}(2))$, the equilibrium shown in Figure 1.3.1 can be simplified to:

$\mathrm{A}+2 \mathrm{C} \stackrel{\mathrm{K}_{\mathrm{AC}}}{\rightleftharpoons} \mathrm{AC}+\mathrm{C} \stackrel{\mathrm{K}_{\mathrm{AC}}}{\rightleftharpoons} \mathrm{AC}_{2}$

where the macroscopic equilibrium constants $\left(\mathrm{K}_{\mathrm{AC}}\right.$ and $\left.\mathrm{K}_{\mathrm{AC}_{2}}\right)$ are given by:

$$
\begin{aligned}
& \mathrm{K}_{\mathrm{AC}}=\mathrm{k}_{1}+\mathrm{k}_{2}=\frac{[\mathrm{AC}(1)]+[\mathrm{AC}(2)]}{[\mathrm{A}][\mathrm{C}]}=\frac{[\mathrm{AC}]}{[\mathrm{A}][\mathrm{C}]} \\
& \mathrm{K}_{\mathrm{AC}_{2}}=\frac{\mathrm{k}_{1} \mathrm{k}_{12}}{\mathrm{k}_{1}+\mathrm{k}_{2}}=\frac{\mathrm{k}_{2} \mathrm{k}_{21}}{\mathrm{k}_{1}+\mathrm{k}_{2}}=\frac{\left[\mathrm{AC}_{2}(1,2)\right]}{([\mathrm{AC}(1)+[\mathrm{AC}(2)])[\mathrm{C}]}=\frac{\left[\mathrm{AC}_{2}\right]}{[\mathrm{AC}][\mathrm{C}]}
\end{aligned}
$$

The macroscopic equilibrium constants do not describe a specific interaction, but the sum of every possible interaction between the analyte and the additive at a particular stoichiometry.

There will also be a macroscopic mobility for the complex AC $\left(\mu_{\mathrm{ep}, \mathrm{AC}}\right)$ :

$$
\mu_{\mathrm{ep}, \mathrm{AC}}=\frac{\mathrm{k}_{1} \mu_{\mathrm{ep}, \mathrm{AC}(1)}+\mathrm{k}_{2} \mu_{\mathrm{ep}, \mathrm{AC}(2)}}{\mathrm{k}_{1}+\mathrm{k}_{2}}=\frac{[\mathrm{AC}(1)] \mu_{\mathrm{ep}, \mathrm{AC}(1)}+[\mathrm{AC}(2)] \mu_{\mathrm{ep}, \mathrm{AC}(2)}}{[\mathrm{AC}(1)]+[\mathrm{AC}(2)]}
$$

The macroscopic complex mobility for $\mathrm{AC}$ is a weighted average of the microscopic mobilities. For $\mathrm{AC}_{2}$, the macroscopic and microscopic mobilities are the same because there is only one analyte species with a 1:2 stoichiometry.

Replacing the microscopic constants in eq 1.3 .7 with the macroscopic ones gives:

$$
v \mu_{\mathrm{ep}}^{\mathrm{A}}=\frac{\mu_{\mathrm{ep}, \mathrm{A}}+\mu_{\mathrm{ep}, \mathrm{AC}} \mathrm{K}_{\mathrm{AC}}[\mathrm{C}]+\mu_{\mathrm{ep}, \mathrm{AC}} \mathrm{K}_{\mathrm{AC}} \mathrm{K}_{\mathrm{AC}}[\mathrm{C}]^{2}}{1+\mathrm{K}_{\mathrm{AC}}[\mathrm{C}]+\mathrm{K}_{\mathrm{AC}} \mathrm{K}_{\mathrm{AC}_{2}}[\mathrm{C}]^{2}}
$$

Equation 1.3.12 describes the effect of analyte-additive interactions with both 1:1 and 1:2 stoichiometries on the net mobility of an analyte. This equation can be derived without considering the microscopic constants but the procedure presented here illustrates how the 
microscopic constants are combined, thereby providing a clearer physical interpretation of the macroscopic constants. In most cases, the mobility of the free analyte can be measured directly, allowing eq 1.3 .12 to be rearranged to:

$$
\left(v \mu_{\mathrm{ep}}^{\mathrm{A}}-\mu_{\mathrm{ep}, \mathrm{A}}\right)=\frac{\left(\mu_{\mathrm{ep}, \mathrm{AC}}-\mu_{\mathrm{ep}, \mathrm{A}}\right) \mathrm{K}_{\mathrm{AC}}[\mathrm{C}]+\left(\mu_{\mathrm{ep}, \mathrm{AC}}-\mu_{\mathrm{ep}, \mathrm{A}}\right) \mathrm{K}_{\mathrm{AC}} \mathrm{K}_{\mathrm{AC}}[\mathrm{C}]^{2}}{1+\mathrm{K}_{\mathrm{AC}}[\mathrm{C}]+\mathrm{K}_{\mathrm{AC}} \mathrm{K}_{\mathrm{AC}}[\mathrm{C}]^{2}}
$$

Equation 1.3 .13 is used to fit the data when both $1: 1$ and $1: 2$ binding stoichiometries are present. When only $1: 1$ binding is present, eq 1.3 .13 can be simplified to:

$$
\left(v \mu_{e p}^{\mathrm{A}}-\mu_{\mathrm{ep}, \mathrm{A}}\right)=\frac{\left(\mu_{\mathrm{ep}, \mathrm{AC}}-\mu_{\mathrm{ep}, \mathrm{A}}\right) \mathrm{K}_{\mathrm{AC}}[\mathrm{C}]}{1+\mathrm{K}_{\mathrm{AC}}[\mathrm{C}]}
$$

\subsubsection{Experimental}

Separations were performed on a Beckman P/ACE 5000 automated CE system (Beckman Instruments Inc., Mississauga, ON, Canada). Uncoated fused silica capillaries (Polymicro Technologies, Phoenix, AZ) were used with inner diameters of $50 \mu \mathrm{m}$, outer diameters of 375 $\mu \mathrm{m}$, and lengths of $57 \mathrm{~cm}$. The length from the injection end to the detector (effective length) was $50 \mathrm{~cm}$. New capillaries were rinsed for 20 minutes with aqueous $1 \mathrm{M}$ sodium hydroxide (BDH Inc., Toronto, ON, Canada) followed by deionized water for five minutes and separation buffer for 20 minutes. The capillary was then allowed to equilibrate overnight before being used. A five minute rinse of buffer was performed immediately before each separation. The sample was introduced using a five second pressure injection $(0.5 \mathrm{psi})$. Separations took place at a potential of $30 \mathrm{kV}$ and a temperature of $20^{\circ} \mathrm{C}$. A Beckman UV-visible detector was used to monitor absorbance at $200 \mathrm{~nm}$. Data were collected and processed using System Gold software (Beckman) and a 486 PC computer. The buffer viscosity was measured by injecting (10 seconds $0.5 \mathrm{psi}$ ) a sample plug onto the capillary and measuring the time needed to push the plug to the 
detector with a constant pressure $(20 \mathrm{psi})^{(35)}$. All regressions were performed using the least squares variance-covariance method (see Appendix I) in Microsoft Excel 5.0.

A stock solution of $20 \mathrm{mM}$ sodium phosphate dibasic (Fisher Scientific, Fair Lawn, NJ, U.S.A.) was prepared in deionized water. The $\mathrm{pH}$ of the phosphate solution was adjusted to 11.0 with $1 \mathrm{M}$ sodium hydroxide before the solution was made up to its final volume. The $\mathrm{pH}$ of the final solution was then confirmed. A $200 \mathrm{mM}$ HP- $\beta-\mathrm{CD}$ (Aldrich Chemical Co., Milwaukee, WI, U.S.A.) stock solution was prepared by dissolving $13.8 \mathrm{~g}$ of the cyclodextrin in $50.00 \mathrm{~mL}$ of the phosphate stock solution. The appropriate ratios of stock solutions were mixed to give separation buffers containing $20 \mathrm{mM}$ phosphate $(\mathrm{pH}=11)$ and $0-200 \mathrm{mM}$ HP- $\beta-\mathrm{CD}$. A stock solution containing approximately $10^{-2} \mathrm{M}$ phenol $\left(\mathrm{pK}_{\mathrm{a}}=10.0 ; 25^{\circ} \mathrm{C} \text { in } \mathrm{H}_{2} \mathrm{O}\right)^{(74)}, 4,4^{\prime}$-biphenol $\left(\mathrm{pK}_{\mathrm{a} 1}=10.4, \mathrm{pK}_{\mathrm{a} 2}=11.7 ; 20^{\circ} \mathrm{C} \text { in } 50 \% \mathrm{MeOH}-\mathrm{H}_{2} \mathrm{O}\right)^{(75)}$, and 4-phenylphenol $\left(\mathrm{pK}_{\mathrm{a}}=9.55 ; 23^{\circ} \mathrm{C}\right.$ in $\left.\mathrm{H}_{2} \mathrm{O}\right)^{(75)}$ (Sigma Chemical Co., St. Louis, MO, U.S.A.) was prepared in methanol. This stock solution was diluted 500 fold in separation buffer to give a sample concentration of $2 \times 10^{-5} \mathrm{M}$. The sample solution then contained $0.2 \%$ methanol, which was used as an EOF marker.

\subsubsection{Binding Isotherms}

The effect of the analyte-additive interaction on analyte migration behavior can be obscured by other factors that also affect the mobility of the analyte. The most important of these factors is the viscosity of the buffer because it often changes with the additive concentration. It can be seen from the following equation that the electrophoretic mobility of an analyte is inversely proportional to the viscosity $(\eta)$ of the separation media.

$$
\mu_{\mathrm{ep}}=\frac{\mathrm{Q}_{\mathrm{eff}}}{6 \pi \eta \mathrm{R}}
$$

Where $Q_{\text {eff }}$ is the effective charge, and $\mathrm{R}$ is the radius of the analyte. When an additive is used, 
the viscosity of the buffer may be affected. This can be accounted for by introducing a correction factor $v$ to normalize the experimental electrophoretic mobilities $\left(v=\eta / \eta^{\circ}\right.$, where $\eta$ is the viscosity of the buffer and $\eta^{\circ}$ is the viscosity when the additive concentration approaches zero). Using this correction factor allows the effect of the complexation to be studied independently of changes in viscosity, which affect the electrophoretic mobility. The viscosity correction factor for the buffer system used in this study was found to be related to the concentration of HP- $\beta-C D$ through a third order polynomial $\left(\nu=199.37[\mathrm{HP}-\beta-\mathrm{CD}]^{3}\right.$ $6.4886[\mathrm{HP}-\beta-\mathrm{CD}]^{2}+6.1952[\mathrm{HP}-\beta-\mathrm{CD}]+1.0092$, where the concentration of HP- $\beta-\mathrm{CD}$ is in molar; $\left.\mathrm{R}^{2}=0.9989\right)$. Over the range of additive concentrations tested $(0-200 \mathrm{mM})$, the viscosity increased 3.5 fold.

The free mobility of the analyte $\left(\mu_{\mathrm{ep}, \mathrm{A}}\right)$ is measured independently, allowing the constants in eqs 1.3 .13 (1:1 and 1:2 binding stoichiometries) and 1.3.14 (only 1:1 binding) to be calculated using nonlinear regressions of $\left(v \mu_{\mathrm{ep}}^{\mathrm{A}}-\mu_{\mathrm{cp}, \mathrm{A}}\right)$ vs. [HP- $\left.\beta-\mathrm{CD}\right]$. The least-squares variancecovariance method is used to perform the regressions. Figure 1.3.2 compares the experimental data to the regression curves calculated according to the equations with and without the presence of 1:2 interactions. Table 1.3.1 lists the equilibrium constants and mobilities obtained from each fitting method. Clearly the equation which considers both $1: 1$ and 1:2 interactions (eq 1.3.13) better describes the experimental data for 4,4'-biphenol and 4-phenylphenol, supporting the premise that these analytes can bind one or two HP- $\beta-C D$ molecules. Higher order equilibria were expected because there are two binding sites available on each of these analytes. It is interesting to note that the regressions made according to eq 1.3 .13 and eq 1.3 .14 both yield very high values for $\mathrm{R}^{2}$. A high correlation coefficient alone is not sufficient to determine what model best describes the data. Visual examinations of the binding isotherms should always be made to determine if the chosen equation accurately describes the data. If the wrong model is chosen, the 
equilibrium constants and complex mobilities calculated from the regression will be misleading.

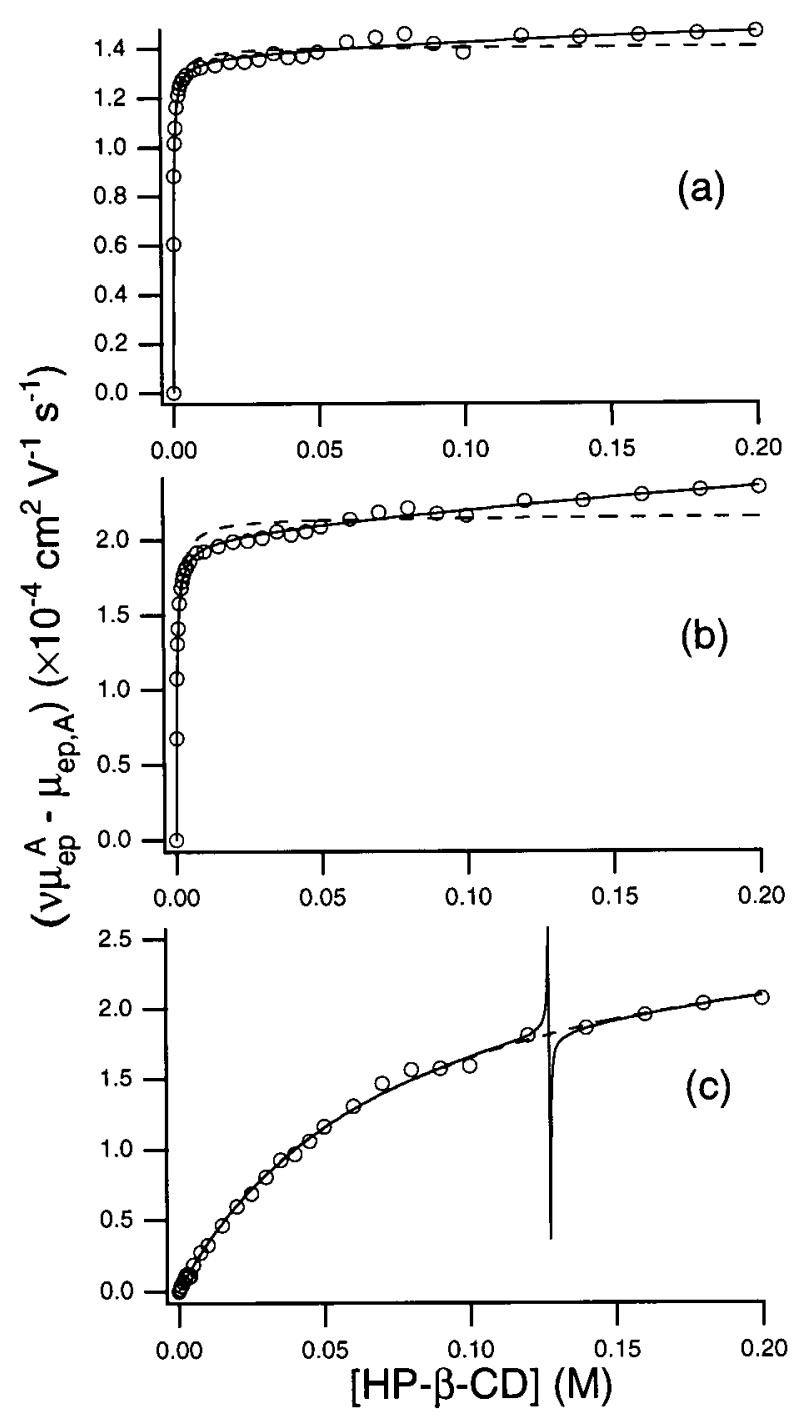

Figure 1.3.2 Binding isotherms for 4-phenylphenol (a), 4,4'-biphenol (b), and phenol (c). The dashed lines were fitted assuming a 1:1 stoichiometry (eq 1.3.14). The solid lines were fitted considering both $1: 1$ and $1: 2$ stoichiometries (eq 1.3.13).

The equation for first order (1:1) binding (eq 1.3.14) best explains the data for phenol. A 1:1 stoichiometry was expected because there is only one binding site on phenol. Nonsensical values, such as a negative equilibrium constant (see Table 1.3.1) and a positive complex mobility $\left(+4 \times 10^{-4} \mathrm{~cm}^{2} \mathrm{~V}^{-1} \mathrm{~s}^{-1}\right.$ for $\left.\mu_{\mathrm{ep}, \mathrm{AC}}\right)$, are obtained when the data for phenol are fitted according to eq 1.3.13, indicating that the $1: 2$ binding stoichiometry should not be included in the regression model for phenol. The discontinuity in the isotherm when the data for phenol are fit according to 
eq 1.3.13 is the result of the additional variables in the regression process when a non-existing $1: 2$ interaction is assumed to be present.

Although the shape of the binding isotherms for interactions with both $1: 1$ and $1: 2$ stoichiometries appear similar in shape to those in which only 1:1 interactions occur, there are some significant differences. When an equation assuming a 1:1 stoichiometry is used to fit data in which 1:2 interactions are present, the resulting isotherm often overestimates the change in analyte mobility when the additive concentrations are low, and underestimates the change when the additive concentrations are high.

Table 1.3.1 Equilibrium constants and electrophoretic mobilities calculated for 4-phenylphenol, 4,4'-biphenol, and phenol using equations assuming a 1:1 or 1:1 and 1:2 complexation stoichiometry

\begin{tabular}{|c|c|c|c|c|c|c|}
\hline & $\begin{array}{l}\mathrm{K}_{\mathrm{AC}} \\
\left(\mathrm{M}^{-1}\right)\end{array}$ & $\begin{array}{l}\mathrm{K}_{\mathrm{Ac}_{2}} \\
\left(\mathrm{M}^{-1}\right)\end{array}$ & $\begin{array}{c}\mu_{\mathrm{ep}, \mathrm{A}} \\
\left(\times 10^{-4} \mathrm{~cm}^{2} \mathrm{~V}^{-1} \mathrm{~s}^{-1}\right)\end{array}$ & $\begin{array}{c}\mu_{\mathrm{gp}, \mathrm{AC}} \\
\left(\times 10^{-4} \mathrm{~cm}^{2} \mathrm{~V}^{-1} \mathrm{~s}^{-1}\right)\end{array}$ & $\begin{array}{c}\mu_{\mathrm{ep}, A c_{2}} \\
\left(\times 10^{-4} \mathrm{~cm}^{2} \mathrm{~V}^{-1} \mathrm{~s}^{-1}\right)\end{array}$ & $\mathrm{R}^{2}$ \\
\hline $\begin{array}{l}\text { 4-Phenylphenol } \\
1: 1 \text { and } 1: 2 \\
1: 1\end{array}$ & $\begin{array}{l}3600 \pm 200 \\
3100 \pm 200\end{array}$ & $3 \pm 5$ & $\begin{array}{l}-2.081 \pm 0.009 \\
-2.081 \pm 0.009\end{array}$ & $\begin{array}{c}-0.7 \pm 0.2 \\
-0.67 \pm 0.01\end{array}$ & $-0.4 \pm 5$ & $\begin{array}{l}0.9987 \\
0.9959\end{array}$ \\
\hline $\begin{array}{l}\text { 4,4'-Biphenol } \\
1: 1 \text { and } 1: 2 \\
1: 1\end{array}$ & $\begin{array}{l}2400 \pm 100 \\
1800 \pm 200\end{array}$ & $2 \pm 2$ & $\begin{array}{l}-3.27 \pm 0.01 \\
-3.27 \pm 0.01\end{array}$ & $\begin{array}{c}-1.3 \pm 0.2 \\
-1.11 \pm 0.03\end{array}$ & $\begin{array}{c}0.2 \pm 6 \\
-\end{array}$ & $\begin{array}{l}0.9990 \\
0.9880\end{array}$ \\
\hline $\begin{array}{l}\text { Phenol } \\
1: 1 \text { and } 1: 2 \\
1: 1\end{array}$ & $\begin{array}{c}5.4 \pm 0.7 \\
13.4 \pm 0.2\end{array}$ & $-19 \pm 3$ & $\begin{array}{l}-2.70 \pm 0.01 \\
-2.70 \pm 0.01\end{array}$ & $\begin{array}{c}4 \pm 1 \\
0.18 \pm 0.06\end{array}$ & $0.2 \pm 0.6$ & $\begin{array}{l}0.9988 \\
0.9985\end{array}$ \\
\hline
\end{tabular}

If phenol and 4-phenylphenol both bear a -1 charge, the complex mobilities listed in Table 1.3.1 do not follow the expected trend of charge to size ratio. The mobilities of the analyte-additive complexes can be explained by considering the acid-base equilibria that both analytes are involved in. HP- $\beta-C D$ has a much stronger affinity towards phenol than phenolate, which shifts the acid-base equilibrium towards phenol. This reduces the apparent mobility of the phenol-HP- $\beta-C D$ complex to near zero. The affinities of 4-phenylphenol and 4-phenylphenolate towards HP- $\beta-C D$ are similar because the analyte is large enough to interact with the cyclodextrin without the negative charge entering the hydrophobic cavity. Therefore, the 
apparent mobility of the 4-phenylphenol-HP- $\beta-C D$ complex is less affected by the acid-base equilibrium. Secondary interactions, such as acid-base equilibria or analyte-solvent interactions almost always influence analyte-additive interactions in CE. These interactions act in concert to determine the apparent analyte mobilities and equilibrium constants. Therefore, these constants can be compared only if the other conditions are kept the same. The effect of multiple equilibria will be discussed further in chapter 1.4. The slightly positive mobility of the phenol-HP- $\beta-C D$ complex is the result of error propagation. The method for estimating the error in the constants when a nonlinear regression is used is not rigorous ${ }^{(73)}$. In this case the error was probably underestimated and the true mobility of the phenol-HP- $\beta-\mathrm{CD}$ complex is near zero.

\subsubsection{Detecting Higher Order Equilibria}

As stated in the previous section, a high correlation coefficient alone is not sufficient to determine if the chosen model accurately depicts the experimental data. The presence of higher order interactions can be confirmed in absorbance or NMR binding studies by transforming the binding isotherm into one of three linear forms ${ }^{(72,73,76)}$. The linear transformations have never before been applied to a CE separation where higher order interactions are present. The linear transformations for the 1:1 stoichiometry binding isotherm (eq 1.3.14) in $\mathrm{CE}^{(62,66)}$ are:

$$
\begin{aligned}
& \frac{1}{\left(v \mu_{\mathrm{ep}}^{\mathrm{A}}-\mu_{\mathrm{ep}, \mathrm{A}}\right)}=\frac{1}{\left(\mu_{\mathrm{ep}, \mathrm{AC}}-\mu_{\mathrm{ep}, \mathrm{A}}\right) \mathrm{K}} \frac{1}{[\mathrm{C}]}+\frac{1}{\left(\mu_{\mathrm{ep}, \mathrm{AC}}-\mu_{\mathrm{ep}, \mathrm{A}}\right)} \\
& \frac{[\mathrm{C}]}{\left(v \mu_{\mathrm{ep}}^{\mathrm{A}}-\mu_{\mathrm{ep}, \mathrm{A}}\right)}=\frac{[\mathrm{C}]}{\left(\mu_{\mathrm{ep}, \mathrm{AC}}-\mu_{\mathrm{ep}, \mathrm{A}}\right)}+\frac{1}{\left(\mu_{\mathrm{ep}, \mathrm{AC}}-\mu_{\mathrm{ep}, \mathrm{A}}\right) \mathrm{K}} \\
& \frac{\left(v \mu_{\mathrm{ep}}^{\mathrm{A}}-\mu_{\mathrm{ep}, \mathrm{A}}\right)}{[\mathrm{C}]}=-\mathrm{K}\left(v \mu_{\mathrm{ep}}^{\mathrm{A}}-\mu_{\mathrm{ep}, \mathrm{A}}\right)+\mathrm{K}\left(\mu_{\mathrm{ep}, \mathrm{AC}}-\mu_{\mathrm{ep}, \mathrm{A}}\right)
\end{aligned}
$$

Plots made according to these equations have acquired different names in different research areas 
but can generally be referred to as double reciprocal (eq 1.3.16), $y$-reciprocal (eq 1.3.17), and $x$ reciprocal (eq 1.3.18)(48,66,73). It is commonly assumed that if the data are linear when plotted according to any one of these equations, the interaction takes place with a 1:1 stoichiometry. This has been done in many cases without considering the effect higher order equilibria have on these plots.

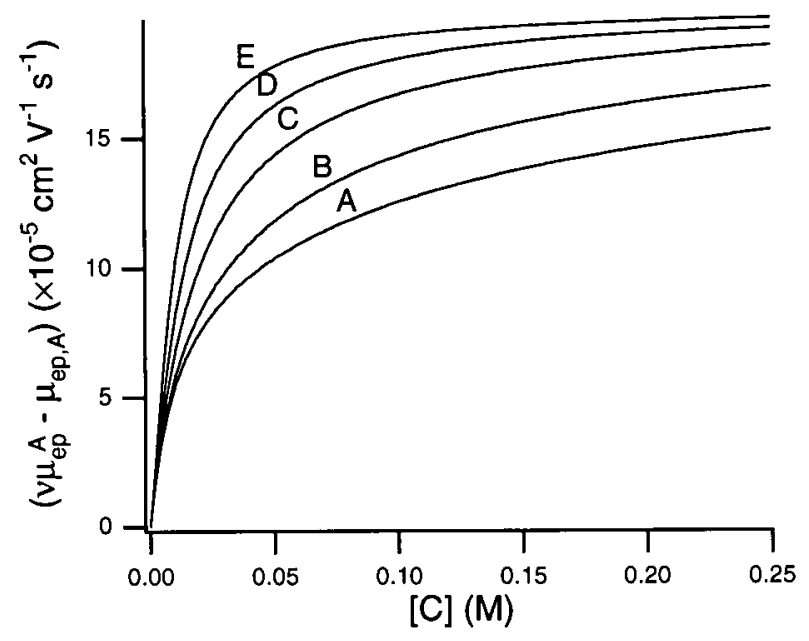

Figure 1.3.3 Hypothetical binding isotherms calculated using the equilibrium constants listed in Table 1.3.2. All the curves were calculated using the following mobilities: $\mu_{\mathrm{gp}, \mathrm{A}}=-2.5 \times 10^{-4} \mathrm{~cm}^{2} \mathrm{~V}^{-1} \mathrm{~s}^{-1} ; \mu_{\mathrm{ep}, \mathrm{AC}}=$ $-1.0 \times 10^{-4} \mathrm{~cm}^{2} \mathrm{~V}^{-1} \mathrm{~s}^{-1} ; \mu_{\mathrm{gp}, A c_{2}}=-0.5 \times 10^{-4} \mathrm{~cm}^{2} \mathrm{~V}^{-1} \mathrm{~s}^{-1}$.

Table 1.3.2 Microscopic and macroscopic equilibrium constants used to generate the curves in Figures 1.3.3 and 1.3.4

\begin{tabular}{ccccc}
\hline & $\begin{array}{c}\mathrm{k}_{1}=\mathrm{K}_{2} \\
\left(\mathrm{M}^{-1}\right)\end{array}$ & $\begin{array}{c}\mathrm{k}_{21}=\mathrm{K}_{12} \\
\left(\mathrm{M}^{-1}\right)\end{array}$ & $\begin{array}{c}\mathrm{K}_{\mathrm{Ac}} \\
\left(\mathrm{M}^{-1}\right)\end{array}$ & $\begin{array}{c}\mathrm{K}_{\mathrm{Ac}} \mathrm{K}_{\mathrm{Ac}} \\
\left(\mathrm{M}^{-2}\right)\end{array}$ \\
\hline $\mathrm{A}$ & 50 & 10 & 100 & 500 \\
$B$ & 50 & 20 & 100 & 1000 \\
$\mathrm{C}$ & 50 & 50 & 100 & 2500 \\
$\mathrm{D}$ & 50 & 100 & 100 & 5000 \\
$\mathrm{E}$ & 50 & 200 & 100 & 10000 \\
\hline
\end{tabular}

Figure 1.3.3 shows binding isotherms for a hypothetical system in which both 1:1 and 1:2 interactions are present. The equilibrium constants used to generate these curves are presented in Table 1.3.2. In curve $\mathrm{C}$, the noncooperative case, all of the microscopic equilibrium constants 
are equal ${ }^{(73)}$. Noncooperative binding occurs when the binding sites on the analyte are identical and interact with the additive independently. Curves A and B represent the anticooperative case where the second binding is not as favorable as the first ${ }^{(73)}$. Anticooperativity will often occur when the binding sites are close enough for steric effects to make the second binding difficult. Most higher order interactions encountered in CE will be either anticooperative or noncooperative. Curves $\mathrm{D}$ and $\mathrm{E}$ represent cooperative binding where the first complexation makes binding at the second site more favorable ${ }^{(73)}$. All of the curves in Figure 1.3.3 are similar in shape to $1: 1$ binding isotherms, making it difficult to detect higher order equilibria by visual inspection alone.

Figure 1.3.4 shows the linear transformations of the binding isotherms presented in Figure 1.3.3. If complexation occurred at a 1:1 stoichiometry, each of these plots would be linear. Clearly, deviations from a 1:1 stoichiometry, exhibited as nonlinearities in Figure 1.3.4, are more easily detected in the linear transformations than in the binding isotherms. Figure 1.3.4 also shows that the three linear transformations are not equal in their ability to reveal higher order equilibria. Deviations from a 1:1 binding stoichiometry are most easily detected in $x$ reciprocal plots (Figure 1.3.4 (a)). The $y$-reciprocal plots (Figure 1.3.4 (b)) are very poor at detecting nonlinearity, especially when anticooperative binding occurs, making them unsuitable for detecting higher order equilibria in $\mathrm{CE}$ since most analyte-additive interactions are anticooperative or noncooperative. Similarly, it is difficult to detect nonlinearity in double reciprocal plots (Figure 1.3.4 (c)) if cooperative binding takes place. 
36.
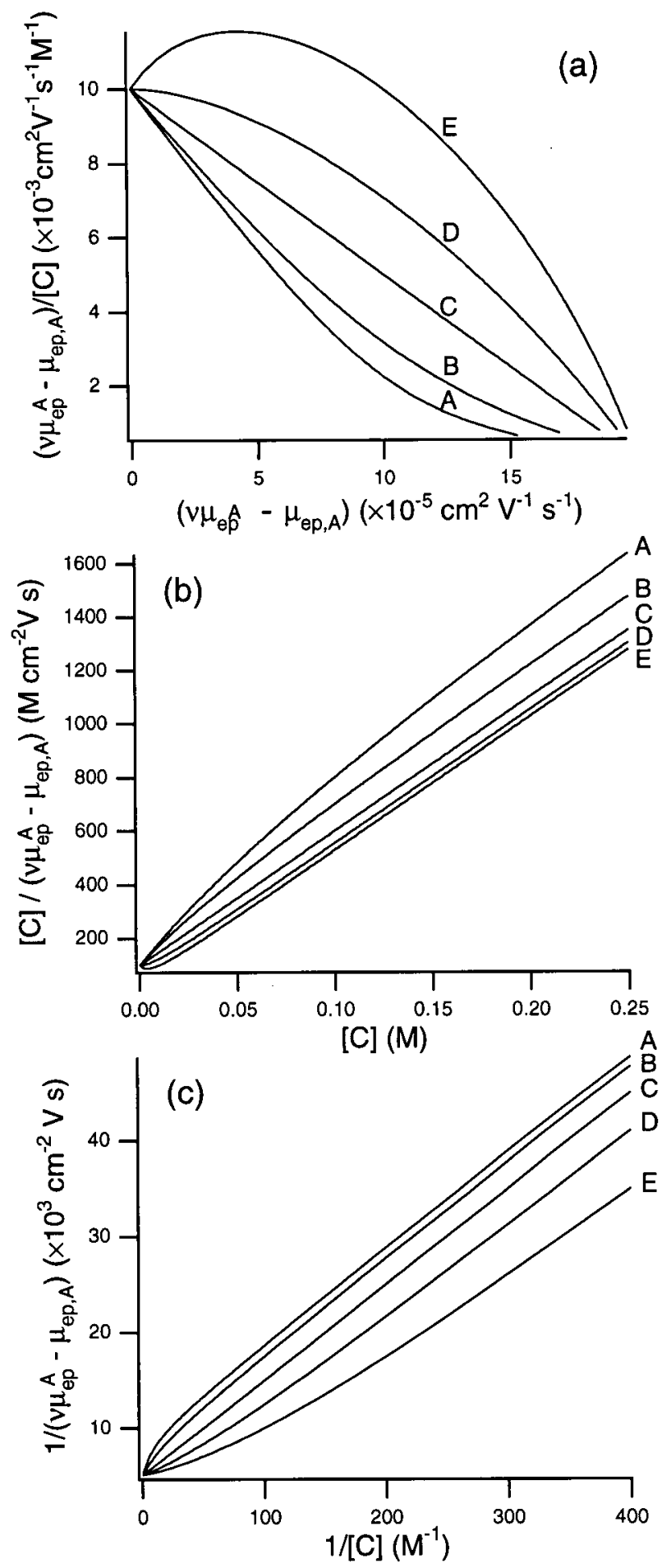

Figure 1.3.4 $x$-Reciprocal plots (a), $y$-reciprocal plots (b), and double reciprocal plots $(\mathrm{c})$ of the binding isotherms in Figure 1.3.3.

It is important to note that when all of the microscopic equilibrium constants are equal $\left(\mathrm{k}_{1}\right.$ $\left.=\mathrm{k}_{2}=\mathrm{k}_{12}=\mathrm{k}_{21}\right)$ and the microscopic complex mobilities are equal $\left(2\left(\mu_{\mathrm{ep}, \mathrm{AC}(1)}-\mu_{\mathrm{ep}, \mathrm{A}}\right)=2\left(\mu_{\mathrm{ep}, \mathrm{AC}(2)}-\right.\right.$ $\left.\mu_{\mathrm{cp}, \mathrm{A}}\right)=\mu_{\mathrm{ep}, \mathrm{Ac}(1,2)}-\mu_{\mathrm{ep}, \mathrm{A}}$, it is impossible to detect higher order equilibria using either the binding 
isotherms or the linear transformations ${ }^{(72)}$. Curve $\mathrm{C}$ in Figures 1.3.4 (a) - (c) corresponds to this special case; all of the linear transformations give a straight line even though complexation takes place with both 1:1 and 1:2 stoichiometries. In practical applications, the microscopic constants do not have to be exactly equal for the linear transformations to appear linear. When the values of the constants are close, random error associated with the data may obscure the curvature in the plot. The experimental error in the data will therefore determine how different the constants have to be in order to observe higher order equilibria. It is therefore impossible, not only in CE, but in complexation chemistry in general, to determine the stoichiometry of an interaction with absolute certainty. At some point it must be accepted that the model sufficiently describes the analyte migration behavior, regardless of whether the actual stoichiometry of the interaction can be proven.

Figure 1.3.5 shows the linear transformations of the data for phenol, 4,4'-biphenol and 4phenylphenol. The plots for phenol are all linear, supporting the premise that it complexes with HP- $\beta$-CD at a 1:1 stoichiometry. The $x$-reciprocal and double reciprocal plots for 4,4'-biphenol and 4-phenylphenol are not linear, indicating that higher order equilibria are taking place. This is the first time that higher order interactions have been detected in CE. It should not be interpreted that higher order interactions are rare in CE since, until now, methods for detecting higher order interactions in CE have not been discussed.

The $y$-reciprocal plots for 4,4'-biphenol and 4-phenylphenol (Figure 1.3.5 (b)) appear to be linear, illustrating that the linear transformations are not equal in their ability to detect higher order equilibria. The linear transformations for 4,4'-biphenol and 4-phenylphenol (Figure 1.3.5 (a) - (c)) most closely resemble the anticooperative curves (A or B) in Figures 1.3.4 (a) - (c). This is expected since $K_{2}$ is much smaller than $K_{1}$ for both 4,4'-biphenol and 4-phenylphenol (see Table 1.3.1) indicating that the second binding is less favorable than the first. Anticooperative binding in this case is likely due to steric effects. 
(a)

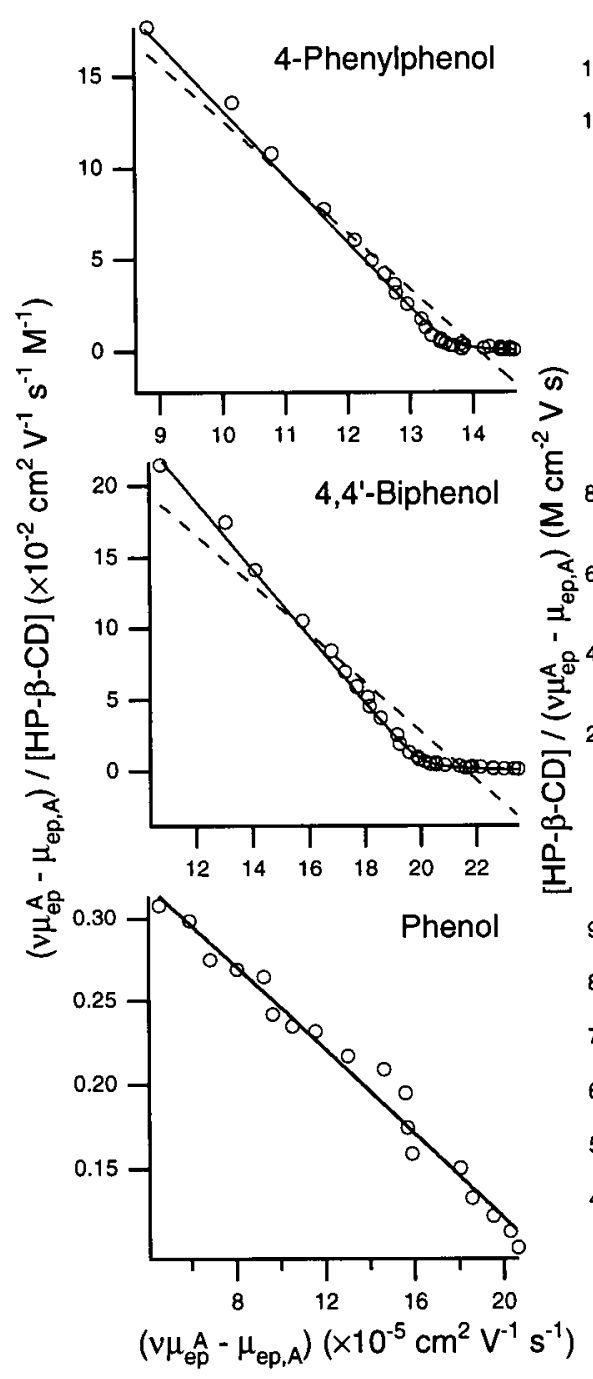

(b)
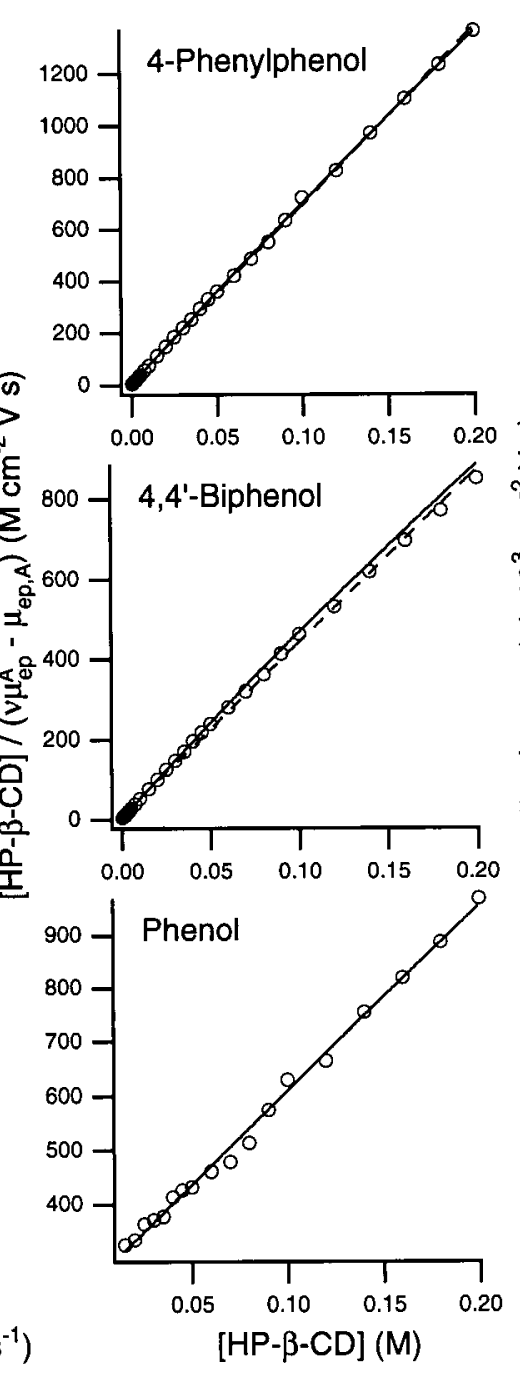

(c)

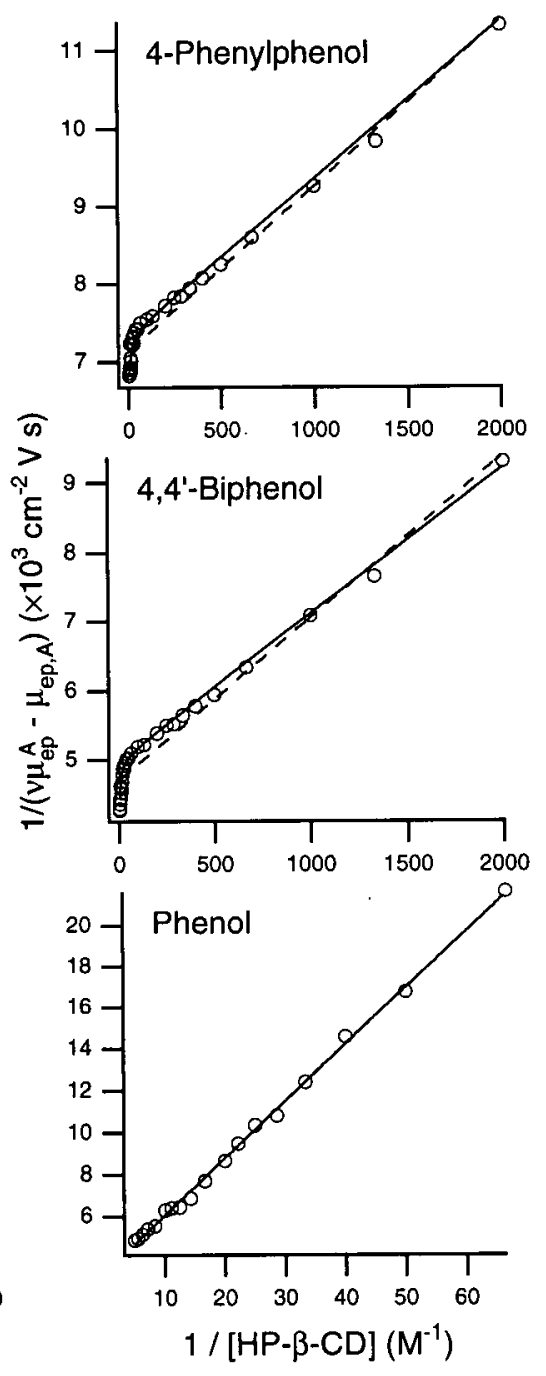

Figure 1.3.5 (a) $x$-Reciprocal plots, (b) $y$-reciprocal plots, and (c) double reciprocal plots for 4-phenylphenol, 4,4'-biphenol, and phenol. The solid lines for 4-phenylphenol and 4,4'-biphenol have been generated using the constants calculated assuming both $1: 1$ and $1: 2$ stoichiometries. The dashed lines are the linear regressions of the data.

Deviations from linearity in the $x$-reciprocal plots for 4-phenylphenol and 4,4'-biphenol become obvious at $[\mathrm{HP}-\beta-\mathrm{CD}]=50 \mathrm{mM}$, indicating that second order binding is already significant at this additive concentration. Changes in temperature, ionic strength and dielectric constant would not be significant until higher additive concentrations. This observation supports the premise that higher order interactions, not systematic errors, cause the deviation from the 1:1 model. 
It has been suggested that when determining if higher order interactions are present, measurements should be made over a certain range of additive concentrations ${ }^{(72,73)}$. Obviously any of the linear transformations will give a straight line if measurements are only made over a small range of additive concentrations. Deranleau(72) used information theory to suggest that at least $75 \%$ of the binding isotherm should be measured in order to achieve results that are representative of the entire concentration range. Connors ${ }^{(73)}$ used the geometric properties of the binding isotherm to recommend a range of $60 \%$. However, in practical applications, no set range of additive concentrations will prove the stoichiometry of an interaction for all conditions. There is always the possibility that higher order equilibria will become significant at higher additive concentrations. For example, using the values in Table 1.3.1, it can be shown that $75 \%$ of $4,4^{\prime}-$ biphenol is singly complexed when the HP- $\beta-C D$ concentration is $1.5 \mathrm{mM}$. At this concentration, only $0.2 \%$ of the 4,4 '-biphenol is doubly complexed and the second order equilibrium will have minimal effect on the overall mobility of the analyte. Even using the $x$ reciprocal plot, nonlinearity is not detectable until the additive concentration reaches $50 \mathrm{mM}$, well above the limits set by Deranleau and Connors. By their criteria this would indicate that a model assuming a 1:1 binding stoichiometry would be sufficient to describe the entire binding isotherm. Figure 1.3.2 clearly shows that this is not the case. Deranleau and Connors presumed that once a $1: 1$ interaction is observed over a certain concentration range, higher order interactions will not occur at any other additive concentration. Higher order interactions, insignificant at lower additive concentrations, may become the dominant factor in determining the analyte mobility at higher additive concentrations. Therefore, when deciding on the range of concentrations over which data is to be collected to prove the stoichiometry of the interaction, the safest course of action is to make measurements over the entire range of interest.

\subsubsection{Additive Concentration Range}

Once the stoichiometry has been determined, the equilibrium constants and complex 
mobilities can be calculated. The range of additive concentrations chosen for the measurements is one of the most important factors in minimizing the error in the calculated constants. This should not be confused with the concentration range used to determine the stoichiometry of the interaction discussed in the previous section. Based on the propagation of error, Deranleau(76) showed that for a system with a 1:1 binding stoichiometry, the error in the calculated equilibrium constant is minimized when data are collected for additive concentrations where the fraction of analyte in the complexed form is between 0.2 and 0.8 . Similarly, the error in the calculated complex mobility will be minimized for data where the fraction of complexation is above 0.2 .

It can be shown that if each equilibrium in the system is treated independently, the concentration ranges calculated by Deranleau also apply to multiple equilibria systems. For example, in a system where both $1: 1$ and 1:2 stoichiometries are present, the error in $\mathrm{K}_{\mathrm{AC}}$ will be minimized when the fraction of analyte complexed with one additive is between 0.2 and 0.8 . The error in $\mu_{\mathrm{ep}, \mathrm{Ac}}$ will be minimized when the fraction of analyte bound to one additive is above 0.2. Similarly, the error in $\mathrm{K}_{\mathrm{AC}} \mathrm{K}_{\mathrm{AC}_{2}}$ will be the lowest when the fraction of the doubly complexed analyte is between 0.2 and 0.8 . The error in $\mu_{\mathrm{ep}, \mathrm{AC}_{2}}$ will be minimized when the fraction of the doubly complexed analyte is above 0.2. Therefore, in order to obtain accurate values for the constants, it is important to make several measurements within the 0.2 to 0.8 range for each interaction.

In some cases, it is impossible to strictly adhere to the ideal 0.2 to 0.8 range of complexation fractions. For systems in which $\mathrm{K}_{\mathrm{AC}_{2}}>\mathrm{K}_{\mathrm{AC}}$, there may not be an additive concentration where the fraction of analyte bound to one additive falls in the range of 0.2 to 0.8 . The solubility of the additive may also limit the range of additive concentrations for which data can be collected. When problems such as these occur, it is important to assess the goal of the experiment. If obtaining accurate estimates of the equilibrium constants and complex mobilities 
is the goal, then the 0.2 to 0.8 range must be strictly adhered to and another technique may be more suitable for measuring the constants. For the purpose of quantitatively describing analyte migration behavior, however, the additive concentration range is less critical. It is important to remember that eqs 1.3 .13 and 1.3 .14 still describe the analyte mobility outside of the 0.2 to 0.8 concentration range. Estimates for the constants are most accurate when measurements are made within this range because the constants have the most effect on the shape of this portion of the binding isotherm. The regions of the binding isotherm outside the 0.2 to 0.8 fraction of complexation are not very sensitive to errors in the equilibrium constant. Therefore, it is not as critical to obtain accurate values of the equilibrium constant to describe the analyte mobility for these concentration ranges. Similarly, when the fraction of complexation is below 0.2 , the shape of the binding isotherm is less dependent on the complex mobility, and an accurate value for the complex mobility is not as critical in predicting the net mobility of the analyte in this region of the binding isotherm. If measurements are made over a certain concentration range, the analyte mobility can always be predicted for that region.

The binding isotherms of 4,4'-biphenol and 4-phenylphenol can be used to illustrate how the range of additive concentrations affects the error in the calculated constants. Because the range of additive concentrations used in this experiment was $0.25 \mathrm{mM}$ to $200 \mathrm{mM}$, the fraction of singly bound $4,4^{\prime}$-biphenol ranged from 0.37 to 0.95 . The fraction of $4,4^{\prime}$-biphenol doubly bound ranged from 0.00015 to 0.24 . The fraction of 4-phenylphenol complexed with one additive ranged from 0.48 to 0.94 , and the fraction of doubly bound 4-phenylphenol ranged from 0.00036 to 0.38 . Most of the measurements were made when the fraction of singly bound 4,4'-biphenol and 4-phenylphenol was between 0.2 and 0.8. For this reason the values for $\mathrm{K}_{\mathrm{AC}}$ and $\mu_{\mathrm{ep}, \mathrm{AC}}$ listed in Table 1.3.1 have reasonably small errors. Only a few measurements were made where the fraction of doubly bound analyte was greater than 0.2. Consequently, the errors in $\mathrm{K}_{\mathrm{AC}}$ and $\mu_{\mathrm{ep}, \mathrm{AC}}$ are relatively high. As stated earlier, although the calculated values of $\mathrm{K}_{\mathrm{AC}}$ and $\mu_{\mathrm{ep}, \mathrm{AC}}$, have a 
high uncertainty associated with them, Figure 1.3.2 shows that they can still be used to accurately predict the data over the range of conditions tested. Measurements made at additive concentrations of 15 to $200 \mathrm{mM}$ were used to calculate $\mathrm{K}_{\mathrm{AC}}$ and $\mu_{\mathrm{ep}, \mathrm{AC}}$ for phenol. The fraction of phenol complexed over these concentrations ranged from 0.17 to 0.73 , which compares well with the recommended limits of 0.2 to 0.8 and explains the relatively low error in $\mathrm{K}_{\mathrm{AC}}$ and $\mu_{\mathrm{ep}, \mathrm{AC}}$.

Another important consideration is the number of measurements required to make accurate estimates of the constants. When using a model that accounts for both $1: 1$ and $1: 2$ stoichiometries (eq 1.3.13), there are four constants that need to be calculated. Therefore, the degrees of freedom will be equal to the number of measurements minus four. To date, most $\mathrm{CE}$ papers considering dynamic complexation processes have used approximately 5-8 measurements. This is not sufficient to detect higher order interactions. We suggest that at least 10 measurements be made if a second order stoichiometry is suspected.

\subsubsection{Capacity Factors and the Fractions of Analyte Species}

Our group has previously demonstrated that the individual capacity factor (eq. 1.2.4) for an interaction with a 1:1 stoichiometry increases linearly with the additive concentration ${ }^{(35)}$. However, the capacity factors for higher order interactions have not been discussed. An interaction with both $1: 1$ and 1:2 stoichiometries can be considered to have three different orders of complexation, i.e. zero order (uncomplexed), first order (singly complexed), and second order (doubly complexed). The individual capacity factors (based on the definition given in eq 1.2.4) for these interactions are:

$$
\begin{aligned}
& k_{\mathrm{A}}^{\prime}=\frac{n_{\mathrm{A}}}{n_{\mathrm{A}}}=\frac{[\mathrm{A}]}{[\mathrm{A}]}=1 \\
& k_{\mathrm{AC}}^{\prime}=\frac{n_{\mathrm{AC}}}{n_{\mathrm{A}}}=\frac{[\mathrm{AC}]}{[\mathrm{A}]}=\mathrm{K}_{\mathrm{AC}}[\mathrm{C}]
\end{aligned}
$$


$k_{\mathrm{AC}_{2}}^{\prime}=\frac{n_{\mathrm{AC}_{2}}}{n_{\mathrm{A}}}=\frac{\left[\mathrm{AC}_{2}\right]}{[\mathrm{A}]}=\mathrm{K}_{\mathrm{AC}} \mathrm{K}_{\mathrm{AC}_{2}}[\mathrm{C}]^{2}$

The order of each interaction with respect to the additive concentration is shown clearly in the capacity factors (eqs 1.3.19-1.3.21). The fraction of analyte present as each species for an interaction with both $1: 1$ and 1:2 stoichiometries can be calculated using the general relation between the fractions and capacity factors given in eq 1.2.9:

$$
\begin{aligned}
& f_{\mathrm{A}}=\frac{k_{\mathrm{A}}^{\prime}}{k_{\mathrm{A}}^{\prime}+k_{\mathrm{AC}}^{\prime}+k_{\mathrm{AC}_{2}}^{\prime}}=\frac{1}{1+\mathrm{K}_{\mathrm{AC}}[\mathrm{C}]+\mathrm{K}_{\mathrm{AC}} \mathrm{K}_{\mathrm{AC}_{2}}[\mathrm{C}]^{2}} \\
& f_{\mathrm{AC}}=\frac{k_{\mathrm{AC}}^{\prime}}{k_{\mathrm{A}}^{\prime}+k_{\mathrm{AC}}^{\prime}+k_{\mathrm{AC}_{2}}^{\prime}}=\frac{\mathrm{K}_{\mathrm{AC}}[\mathrm{C}]}{1+\mathrm{K}_{\mathrm{AC}}[\mathrm{C}]+\mathrm{K}_{\mathrm{AC}} \mathrm{K}_{\mathrm{AC}_{2}}[\mathrm{C}]^{2}} \\
& f_{\mathrm{AC}_{2}}=\frac{k_{\mathrm{AC}_{2}}^{\prime}}{k_{\mathrm{A}}^{\prime}+k_{\mathrm{AC}}^{\prime}+k_{\mathrm{AC}_{2}}^{\prime}}=\frac{\mathrm{K}_{\mathrm{AC}} \mathrm{K}_{\mathrm{AC}_{2}}[\mathrm{C}]^{2}}{1+\mathrm{K}_{\mathrm{AC}}[\mathrm{C}]+\mathrm{K}_{\mathrm{AC}_{\mathrm{AC}}} \mathrm{K}_{\mathrm{AC}_{2}}[\mathrm{C}]^{2}}
\end{aligned}
$$

Eqs 1.3.22-1.3.24 determine the relative amounts of each analyte species at different additive concentrations. Figure 1.3.6 compares the capacity factors and the fractions of analyte complexed at each stoichiometry for 4-phenylphenol over 0 to $250 \mathrm{mM}$ HP- $\beta$-CD. As shown in Figure 1.3.6 (a), the capacity factors of the complexes $\left(\mathrm{AC}\right.$ and $\left.\mathrm{AC}_{2}\right)$ increase with HP- $\beta-\mathrm{CD}$ concentration. However, as Figure 1.3.6 (b) shows, an increase in the capacity factor of a particular species does not necessarily indicate that the fraction of analyte in that species is increasing. The capacity factor only describes relative tendency of the analyte to exist as a specific species in a competitive environment. To determine the actual fraction of analyte in each species, the capacity factors for all of the analyte species need to be considered. 
44.
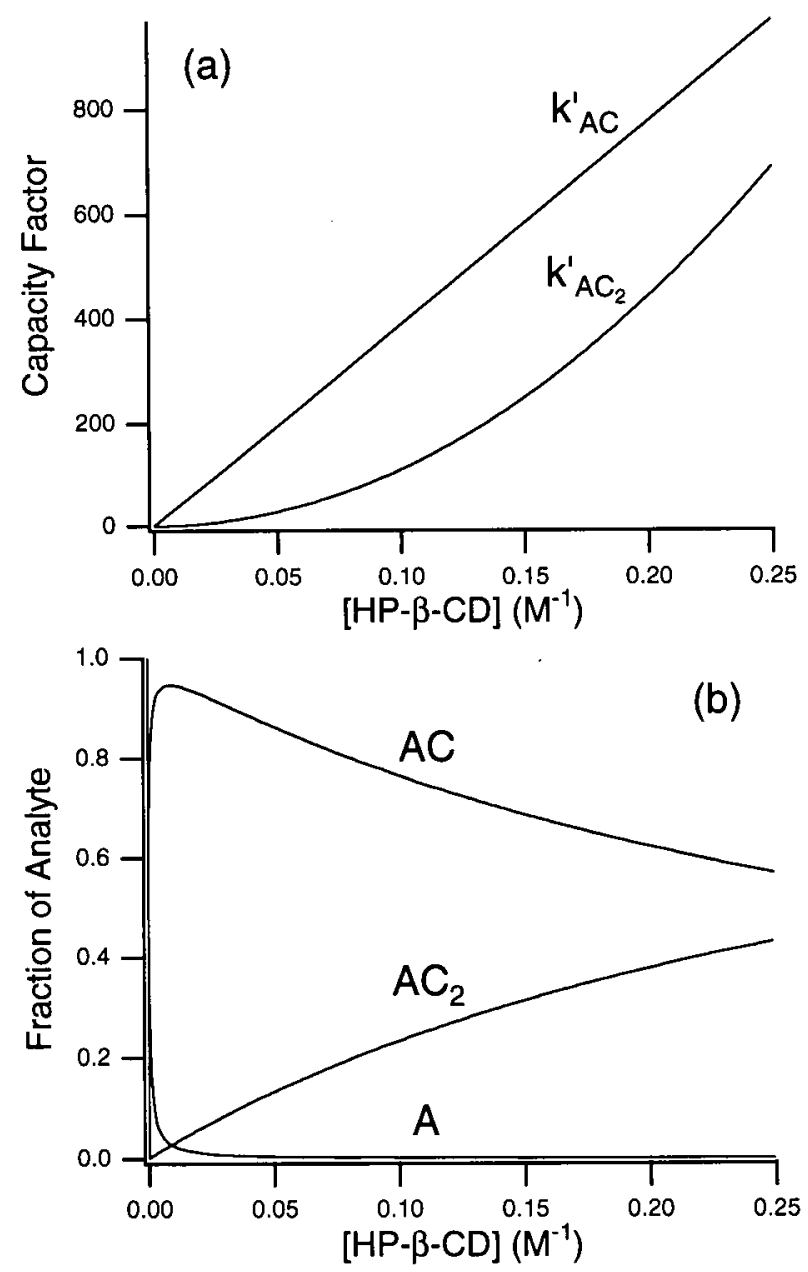

Figure 1.3.6 Comparison of the capacity factors (a) and fractions (b) of free, singly bound, and doubly bound 4-phenylphenol for 0 to $250 \mathrm{mM}$ HP- $\beta-C D$.

The small values of $\mathrm{K}_{\mathrm{AC}_{2}}$ in Table 1.3.1 could give the impression that the second order interaction is insignificant. However, the capacity factor for the second order interaction $\left(\mathrm{K}_{\mathrm{AC}} \mathrm{K}_{\mathrm{AC}_{2}}[\mathrm{C}]^{2}\right)$ increases much faster at higher additive concentrations than the capacity factor of the first order interaction (see Figure 1.3.6(a)). It will be generally true that lower order equilibria are more significant at low additive concentrations and higher order equilibria become more significant at higher additive concentrations. 


\subsubsection{Predicting the Electroosmotic Mobility and Analyte Migration Times}

Figure 1.3.2 shows that eq 1.3.13 accurately describes the electrophoretic mobilities of 4,4'-biphenol and 4-phenylphenol, and eq 1.3.14 accurately describes the electrophoretic mobility of phenol for 0.25 to $200 \mathrm{mM}$ HP- $\beta-\mathrm{CD}$. The accurate description of the analyte mobilities over such a large range of additive concentrations demonstrates both the usefulness and robustness of dynamic complexation theory.

When the electroosmotic mobility is known, the migration times of the analytes can be calculated from the electrophoretic mobilities. Unfortunately, the unpredictability of the EOF often makes it difficult to predict migration times from electrophoretic mobilities. Although the EOF should be inversely proportional to the viscosity, additives often change the $\zeta$-potential at the capillary surface, making it difficult to correlate the electroosmotic mobility with viscosity. This does not seem to be the case with neutral cyclodextrins, as several publications have suggested that the EOF is a good measure of the viscosity for certain buffer systems which use cyclodextrins as additives ${ }^{(62)}$. In the present study, the electroosmotic mobility $\left(\mu_{\mathrm{e}}\right)$ is related to the viscosity correction factor $\left(\mu_{\mathrm{eo}}=[46.06(1 / \mathrm{v})+6.964] \times 10^{-5} \mathrm{~cm}^{2} \mathrm{~V}^{-1} \mathrm{~s}^{-1} ; \mathrm{R}^{2}=0.9984\right)$. Because the viscosity correction factor $(v)$ can be predicted for 0 to $200 \mathrm{mM}$ HP- $\beta-C D$ using a third order polynomial, the electroosmotic mobility can also be predicted. Figure 1.3 .7 compares the actual migration times of the analytes and the EOF marker with the curves predicted using the calculated electrophoretic mobilities, viscosity correction factors, and electroosmotic mobilities. The fit is excellent up to $160 \mathrm{mM}$ HP- $\beta-C D$, indicating that changes in temperature and ionic strength are not significant below this concentration. Above $160 \mathrm{mM}$, the electroosmotic mobilities begin to deviate from the values predicted using the viscosity correction factor, indicating that at high concentrations, HP- $\beta-C D$ does start to affect other properties of the buffer. It should be noted that the EOF for different capillaries could be very different, making it difficult to reproduce results identical to those in Figure 1.3.7. However, if the temperature is the 
same, the electrophoretic mobilities in Figure 1.3.2 and the correlation between the viscosity correction factor and the cyclodextrin concentration should be valid for any capillary in any system. In order to predict the migration times on a different capillary, the correlation between the electroosmotic mobility and the buffer viscosity would have to be recalculated.

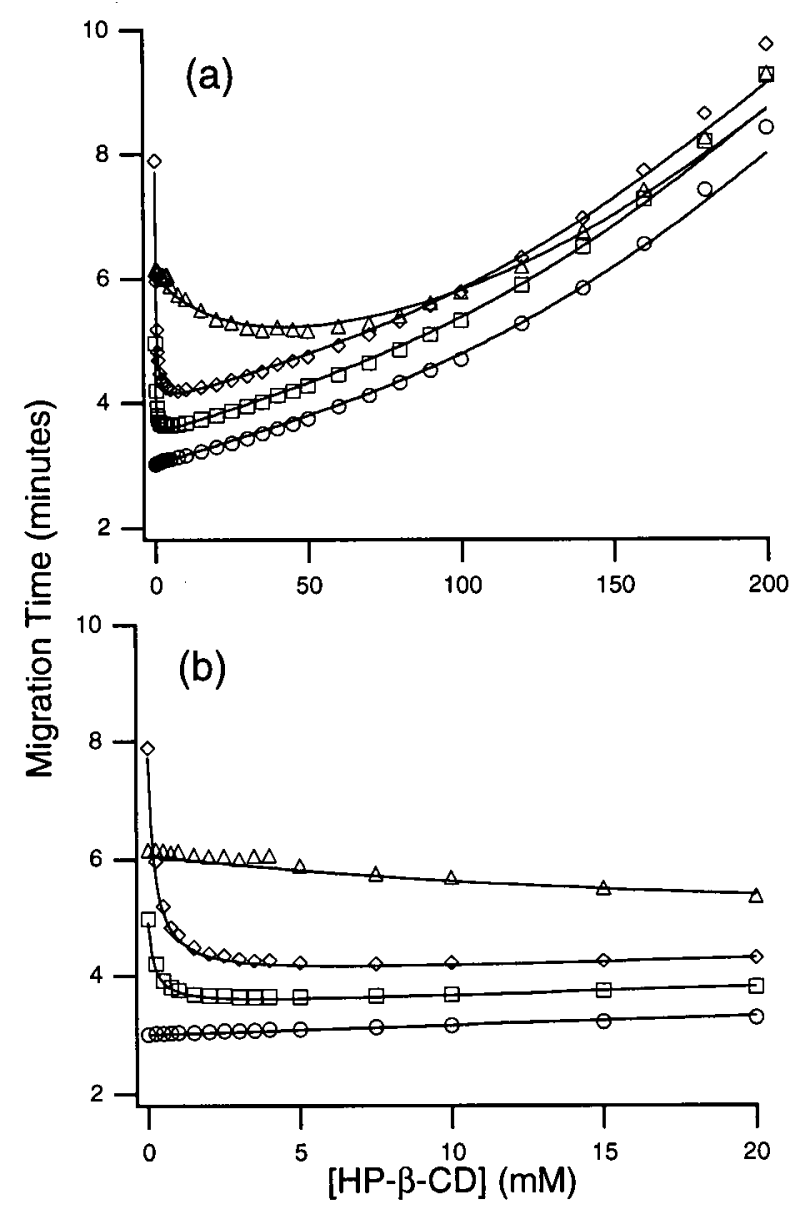

Figure 1.3.7 The migration times predicted using the calculated electrophoretic mobilities, viscosity correction factors, and electroosmotic mobilities for 4-phenylphenol( $\square), 4,4^{\prime}$-biphenol $(\diamond)$, phenol $(\Delta)$, and the EOF marker(O). Plot (b) is an expansion of plot (a).

\subsubsection{Conclusions}

This chapter has presented a generally applicable, quantitative method that describes the effect of higher order equilibria on the migration behavior of analytes in CE. The procedures for detecting higher order interactions and the regression methods for determining the constants necessary to describe the analyte migration behavior were discussed. The relationship between 
the capacity factors and the fraction of analyte present as each species was demonstrated for analyte-additive complexes with stoichiometries other than 1:1. The use of the individual capacity factors should allow the concepts presented in this chapter to be extended to systems with even more complicated equilibria. 
48.

\subsection{The Properties of Multivariate Binding Isotherms in Capillary Electrophoresis}

\subsubsection{Introduction}

Most quantitative CE binding studies only consider systems with a single additive. Recently it has been shown that separations can sometimes be improved if more than one additive is used at the same time(60,77,78). Peng et al. ${ }^{(35)}$ provided the first quantitative description of analyte migration behavior in CE with more than one buffer additive. Kranack et al.(79) expanded on multiple additive CE by demonstrating the usefulness of separation systems utilizing both charged and uncharged cyclodextrins.

Studying multiple equilibria also has important consequences for CE systems in which one or no additives are used. Because CE is always performed in solution, there is always the potential for equilibria to take place. Potential equilibria include analyte-buffer, acid-base, and analyte-solvent interactions as well as any analyte-additive interactions that may be introduced into the system. Therefore, a better understanding of complex equilibrium systems should lead to a better understanding of how secondary interactions affect the mobility of an analyte. The effect secondary equilibria have on the constants estimated in a CE binding study also needs to be considered.

The aim of the following chapter is to discuss the effects of multiple equilibria on multivariate binding isotherms using applied mathematics. Although this is a purely theoretical discussion, comparisons can be made to the experimental binding isotherms presented by Peng $e t$ $a l .(35)$ and Kranack et al..$^{(79)}$

\subsubsection{First Order Binding in the Presence of Two Additives}

When binding occurs at a 1:1 (analyte:additive) stoichiometry with two additives present in a CE system, the analyte migration behavior can be described by the following equation ${ }^{(35)}$ : 
$v \mu_{e p}^{A}=\frac{\mu_{e p, A}+K_{A C}[C] \mu_{e p, A C}+K_{A D}[D] \mu_{e p, A D}}{1+K_{A C}[C]+K_{A D}[D]}$

where $v$ is a correction factor, $\mu_{\mathrm{ep}}^{\mathrm{A}}$ is the net electrophoretic mobility of the analyte, $\mu_{\mathrm{ep}, \mathrm{A}}$ is the mobility of the uncomplexed analyte (A), $\mathrm{C}$ and $\mathrm{D}$ are the additives, $\mathrm{K}_{\mathrm{AC}}$ and $\mathrm{K}_{\mathrm{AD}}$ are the formation constants for the complexes $\mathrm{AC}$ and $\mathrm{AD}$, and $\mu_{\mathrm{ep}, \mathrm{AC}}$ and $\mu_{\mathrm{ep}, \mathrm{AD}}$ are the electrophoretic mobilities of the complexes $\mathrm{AC}$ and $\mathrm{AD}$, respectively. This model (eq 1.4.1) assumes that there are no interactions between additive $\mathrm{C}$ and $\mathrm{D}$, and that the analyte can only bind one of the additives at a time. Also, all equilibria must take place at a fast rate in relation to the overall separation time. In order to observe the phenomena discussed in this section, $\mu_{\mathrm{ep}}^{\mathrm{A}}$ must be corrected to account for any changes in the mobility of the analyte caused by factors other than the equilibrium. The most important factor to consider is the viscosity of the buffer which often varies with the additive concentration.

Equation 1.4.1 describes a binding isotherm surface (See Figure 1.4.1) from which the net mobility of the analyte can be determined for any concentration of the additives C and D. Figure 1.4.1 demonstrates three-dimensional plots of two hypothetical surfaces that have been generated according to eq 1.4.1. Similar three-dimensional plots of experimental data have been presented elsewhere ${ }^{(35,79)}$. While this presentation style is useful for a quick assessment of the shape of the isotherm, other presentation methods emphasize certain properties of the isotherm more clearly. One alternative presentation method is the use of a contour plot. For any isotherm surface described by eq 1.4.1, it is possible to draw contour lines along which $v \mu_{\mathrm{ep}}^{\mathrm{A}}$ remains constant. A contour plot is made by drawing contour curves at constant increments of $v \mu_{\mathrm{ep}}^{\mathrm{A}}$. Contour plots describe the surface in a similar manner to contour maps. Areas where the contour curves are close together represent portions of the surface with a high slope; areas where the contour curves are further apart represent flatter portions of the surface. The contour plots of the 
isotherms in Figure 1.4.1 are shown in Figure 1.4.2.
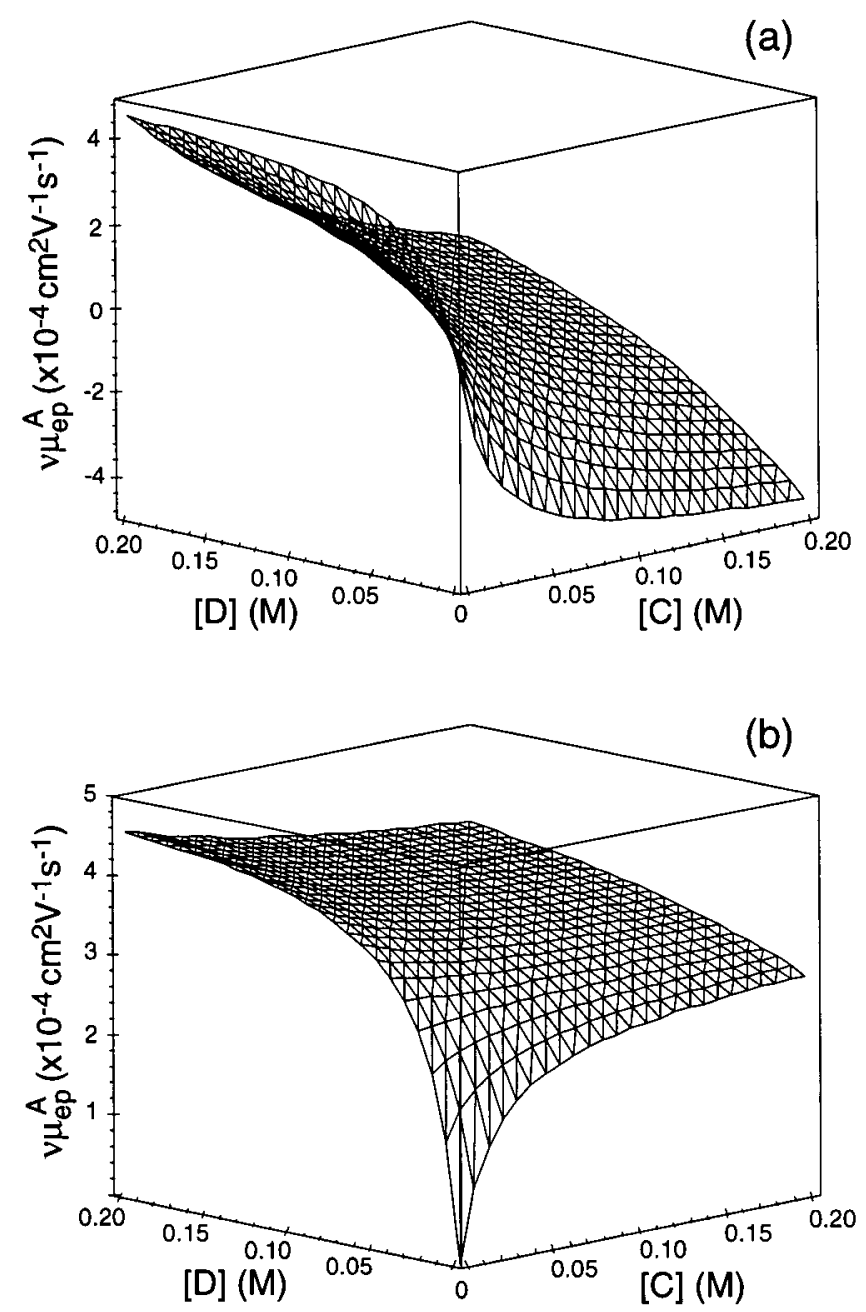

Figure 1.4.1 Three-dimensional plots of binding isotherm surfaces where both additives interact with the analyte with 1:1 binding stoichiometries (eq 1.4.1). The additives shift $v \mu_{\mathrm{ep}}^{\mathrm{A}}$ in opposite directions in (a) and the same direction in (b). The constants used to generate the curves are: $\mathrm{K}_{\mathrm{AC}}=50 \mathrm{M}^{-1} ; \mathrm{K}_{\mathrm{AD}}=50 \mathrm{M}^{-1} ; \mu_{\mathrm{\theta P}, \mathrm{A}}=0 \mathrm{~cm}^{2} \mathrm{~V}^{-1} \mathrm{~s}^{-1} ; \mu_{\mathrm{ep}, \mathrm{AD}}$ $=5 \times 10^{-5} \mathrm{~cm}^{2} V^{-1} \mathrm{~s}^{-1} ; \mu_{\text {ep }, A C}=-5 \times 10^{-5} \mathrm{~cm}^{2} V^{-1} \mathrm{~s}^{-1}(\mathrm{a})$ and $3 \times 10^{-5} \mathrm{~cm}^{2} \mathrm{~V}^{-1} \mathrm{~s}^{-1}$ (b). 
(a)
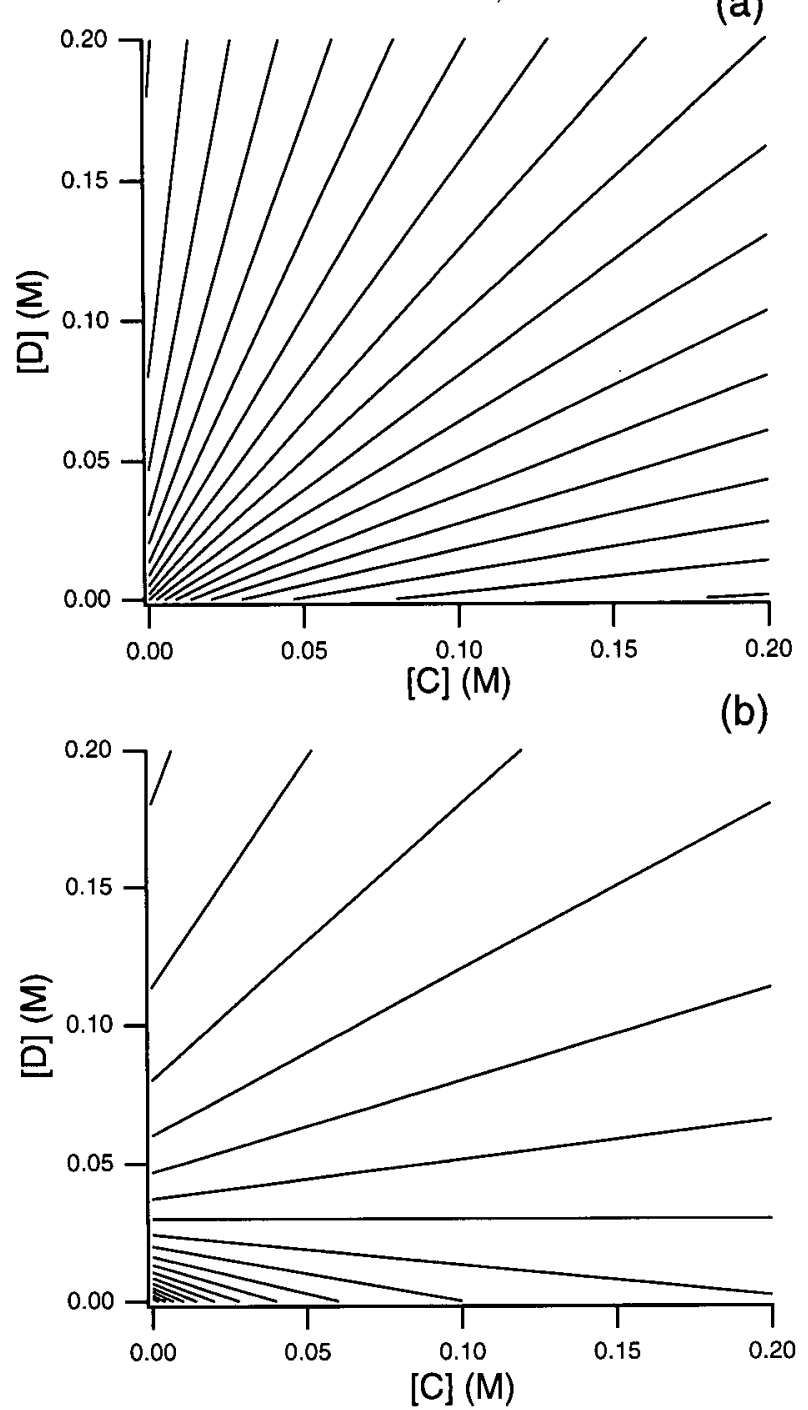

Figure 1.4.2 Contour plots of a binding isotherm surface where both additives interact with the analyte with 1:1 binding stoichiometries (eq 1.4.1). The additives shift $v \mu_{\mathrm{ep}}^{\mathrm{A}}$ in opposite directions in (a) and the same direction in (b). The constants used to generate the curves are the same as those in Figure 1.4.1. In (a), the contour curves range from $v \mu_{\mathrm{ep}}^{\mathrm{A}}=-4.5 \times 10^{-5} \mathrm{~cm}^{2} \mathrm{~V}^{-1} \mathrm{~s}^{-1}$ to $4.5 \times 10^{-5} \mathrm{~cm}^{2} \mathrm{~V}^{-1} \mathrm{~s}^{-1}$ at increments of $0.5 \times 10^{-5}$ $\mathrm{cm}^{2} V^{-1} \mathrm{~s}^{-1}$. In (b), the contour curves range from $v \mu_{\mathrm{ep}}^{\mathrm{A}}=0 \mathrm{~cm}^{2} \mathrm{~V}^{-1} \mathrm{~s}^{-1}$ to $4.5 \times 10^{-5} \mathrm{~cm}^{2} \mathrm{~V}^{-1} \mathrm{~s}^{-1}$ at increments of $0.25 \times 10^{-5} \mathrm{~cm}^{2} \mathrm{~V}^{-1} \mathrm{~s}^{-1}$.

The slope of the contour curve for any $v \mu_{\mathrm{ep}}^{\mathrm{A}}$ is equal to the partial derivative of [D] with respect to $[\mathrm{C}]$ :

$\frac{\partial[D]}{\partial[C]}=-\frac{K_{A C}\left(v \mu_{e p}^{A}-\mu_{e p, A C}\right)}{K_{A D}\left(v \mu_{e p}^{A}-\mu_{e p, A D}\right)}$ 
Because eq 1.4.2 is independent of either [C] or [D], the contour curves for any isotherm described by eq 1.4.1 (at any $v \mu_{\mathrm{ep}}^{\mathrm{A}}$ ) will always be linear, as is shown in Figure 1.4.2.

There are two cases to consider when describing the binding isotherm surfaces for systems with two additives. One case is when the two additives have an opposite effect on the net analyte mobility (i.e. one additive increases the net mobility of the analyte and the other decreases the net analyte mobility), as shown in Figure 1.4.2 (a). The contour curve passing through the origin corresponds to $v \mu_{\mathrm{ep}}^{\mathrm{A}}=\mu_{\mathrm{ep}, \mathrm{A}^{*}}$. It is possible to adjust [C] and [D] along the contour curve without changing the net mobility of the analyte. Other contour lines in Figure 1.4.2 indicate the $[C]$ and $[D]$ coordinate pairs necessary to set $v \mu_{\mathrm{ep}}^{\mathrm{A}}$ to those mobility values. The intercepts of the contour lines are determined by the interaction between the analyte and the individual additives. The analyte mobility can range from $\mu_{\mathrm{ep}, \mathrm{A}}$ to $\mu_{\mathrm{ep}, \mathrm{AC}}$ using additive $\mathrm{C}$ alone, and from $\mu_{\mathrm{ep}, \mathrm{A}}$ to $\mu_{\mathrm{ep}, \mathrm{AD}}$ using only additive $\mathrm{D}$. If both additives are used, $v \mu_{\mathrm{ep}}^{\mathrm{A}}$ can range from $\mu_{\mathrm{ep}, \mathrm{AD}}$ to $\mu_{\mathrm{ep}, \mathrm{AC}}$. According to eq 1.4.2, when the additives shift the net mobility of the analyte in opposite directions, the slope of the contour curves will be positive over the entire range of $v \mu_{e p}^{A}$ values. This is expected because the ranges of mobilities obtainable by each individual additive do not overlap. The mobilities obtained using only additive $\mathrm{C}$ can not be achieved using additive D alone, therefore, it is not possible for a contour line to intersect both the $[C]$ and $[D]$ axes (i.e. have a negative slope). According to eq 1.4.2, for the slope of a contour curve to be zero or infinity (i.e. perpendicular to one of the concentration axes), the net mobility of the analyte must be equal to $\mu_{\mathrm{ep}, \mathrm{AD}}$ or $\mu_{\mathrm{ep}, \mathrm{AC}}$. Although the net analyte mobility is limited by $\mu_{\mathrm{ep}, \mathrm{AD}}$ and $\mu_{\mathrm{ep}, \mathrm{AC}}, v \mu_{\mathrm{ep}}^{\mathrm{A}}$ only equals $\mu_{\mathrm{ep}, \mathrm{AD}}$ or $\mu_{\mathrm{ep}, \mathrm{AC}}$ at infinite concentrations of that additive. For practical purposes, the slope of the contour curves for binding isotherms described by eq 1.4 .1 will not be equal to zero or infinity when the two additives shift the analyte mobility in opposite directions. 
The other case to be considered is illustrated in Figure 1.4.2 (b), where both additives shift the net mobility of the analyte in the same direction. In this example, $\left|\mu_{\mathrm{ep}, \mathrm{AD}}-\mu_{\mathrm{ep}, \mathrm{A}}\right|$ is greater than $\left|\mu_{\text {ep,AC }}-\mu_{\text {ep,A }}\right|$. Because both additives shift the analyte mobility in the same direction, and the value of $\left|\mu_{e p, A D}-\mu_{e p, A}\right|$ is greater than $\left|\mu_{e p, A C}-\mu_{e p, A}\right|, v \mu_{e p}^{A}$ can range from $\mu_{e p, A}$ to $\mu_{e p, A D}$. According to eq 1.4.2, the slope of the contour lines will be negative when $v \mu_{\mathrm{ep}}^{\mathrm{A}}$ is less than $\mu_{\mathrm{ep}, \mathrm{AC}}$. Over this range it is possible to reach a certain $\nu \mu_{\mathrm{ep}}^{\mathrm{A}}$ using either additive alone. Therefore, the contour lines will intersect both the [C] and the [D] axes. When $v \mu_{e p}^{\mathrm{A}}$ is greater than $\mu_{\mathrm{ep}, \mathrm{AC}}$ it is impossible to reach $v \mu_{\mathrm{ep}}^{\mathrm{A}}$ using additive $\mathrm{C}$ alone. Therefore, the contour lines for this range will not cross the $[\mathrm{C}]$ axis. There is a special case when $v \mu_{\mathrm{ep}}^{\mathrm{A}}=\mu_{\mathrm{ep}, \mathrm{AC}}$. According to eq 1.4.2, the slope of the contour line corresponding to $v \mu_{\mathrm{ep}}^{\mathrm{A}}=\mu_{\mathrm{ep}, \mathrm{AC}}$ is equal to zero, indicating that at a certain concentration of $\mathrm{D}$, the net mobility of the analyte will not be affected by the concentration of additive $\mathrm{C}$. This has been referred to previously as a dengsu point ${ }^{(35)}$. Dengsu means "same speed" in Chinese. The additive concentration at which a dengsu point occurs is referred to as the dengsu concentration and is denoted by a subscript $D$ (e.g. $[D]_{D}$ ). At the dengsu concentration, the slope of the binding surface perpendicular to the $[\mathrm{D}]$ axis is equal to zero. The dengsu concentration can therefore be determined by setting the partial derivative of $v \mu_{\mathrm{ep}}^{\mathrm{A}}$ with respect to [C] to zero, and solving for [D]:

$$
\begin{aligned}
& \frac{\partial\left(v \mu_{e p}^{A}\right)}{\partial[C]}=\frac{K_{A C}\left(\mu_{e p, A C}-\mu_{e p, A}\right)+K_{A C} K_{A D}[D]\left(\mu_{e p, A C}-\mu_{e p, A D}\right)}{\left(1+K_{A C}[C]+K_{A D}[D]\right)^{2}}=0 \\
& {[D]_{D}=-\frac{\left(\mu_{e p, A C}-\mu_{e p, A}\right)}{K_{A D}\left(\mu_{e p, A C}-\mu_{e p, A D}\right)}}
\end{aligned}
$$

Equation 1.4 .4 is identical to that proposed by Peng et al. ${ }^{(35)}$. Equation 1.4 .4 will only give a positive concentration when both additives shift the mobility of the analyte in the same direction 
and $\left|\mu_{e p, A D}-\mu_{c p, A}\right|$ is larger than $\left|\mu_{e p, A C}-\mu_{e p, A}\right|$. Conversely, if $\left|\mu_{c p, A C}-\mu_{e p, A}\right|$ is larger than $\left|\mu_{e p, A D}-\mu_{e p, A}\right|$, there will be a dengsu concentration for $\mathrm{C}$. When the two complex mobilities for the additives are equal, according to eq 1.4 .4 the dengsu concentration for both additives will be infinity. This is the only case where there will not be an observable dengsu concentration for one of the additives when binding occurs with a 1:1 stoichiometry. When $[D]$ is higher than the dengsu concentration, increasing [C] causes a decrease in the net mobility of the analyte. This may seem counterintuitive since increasing [C] in the absence of $D$ causes an increase in $v \mu_{\mathrm{ep}}^{\mathrm{A}}$. It is important to remember that when [D] is above the dengsu concentration, $v \mu_{\mathrm{ep}}^{\mathrm{A}}$ is above $\mu_{\mathrm{ep}, \mathrm{AC}}$. Increasing the concentration of an additive always shifts the net mobility of the analyte towards the mobility of that analyte-additive complex. In the case where $[D]$ is above the dengsu concentration, an increase in [C] shifts $v \mu_{\mathrm{ep}}^{\mathrm{A}}$ towards $\mu_{\mathrm{ep}, \mathrm{AC}}$, which decreases the net mobility of the analyte.

Profile plots are obtained by plotting binding isotherms for one additive at constant concentrations of the second additive. If the concentration of the second additive is varied at fixed increments, the result is analogous to viewing the profile of the binding isotherm surface, perpendicular to one of the concentration axes. The dengsu point is easily identified on profile plots. Figure 1.4.3 shows the profile plots of an isotherm surface where both additives increase the net mobility of the analyte (the case shown in Figure 1.4.2(b)). Figure 1.4.3(a) shows the profile of the surface when viewed perpendicular to the $[C]$ axis. Since $\mid \mu_{e p, A C}-\mu_{e p, A} I$ is less than $\mid \mu_{\mathrm{ep}, \mathrm{AD}}-\mu_{\mathrm{ep}, \mathrm{A}} \mathrm{l}$, there is no dengsu concentration for additive $\mathrm{C}$. The dengsu point for additive $\mathrm{D}$ is shown as a horizontal dashed line in Figure 1.4.3(a), demonstrating that the net analyte mobility is not affected by [C] when [D] is at the dengsu concentration. 

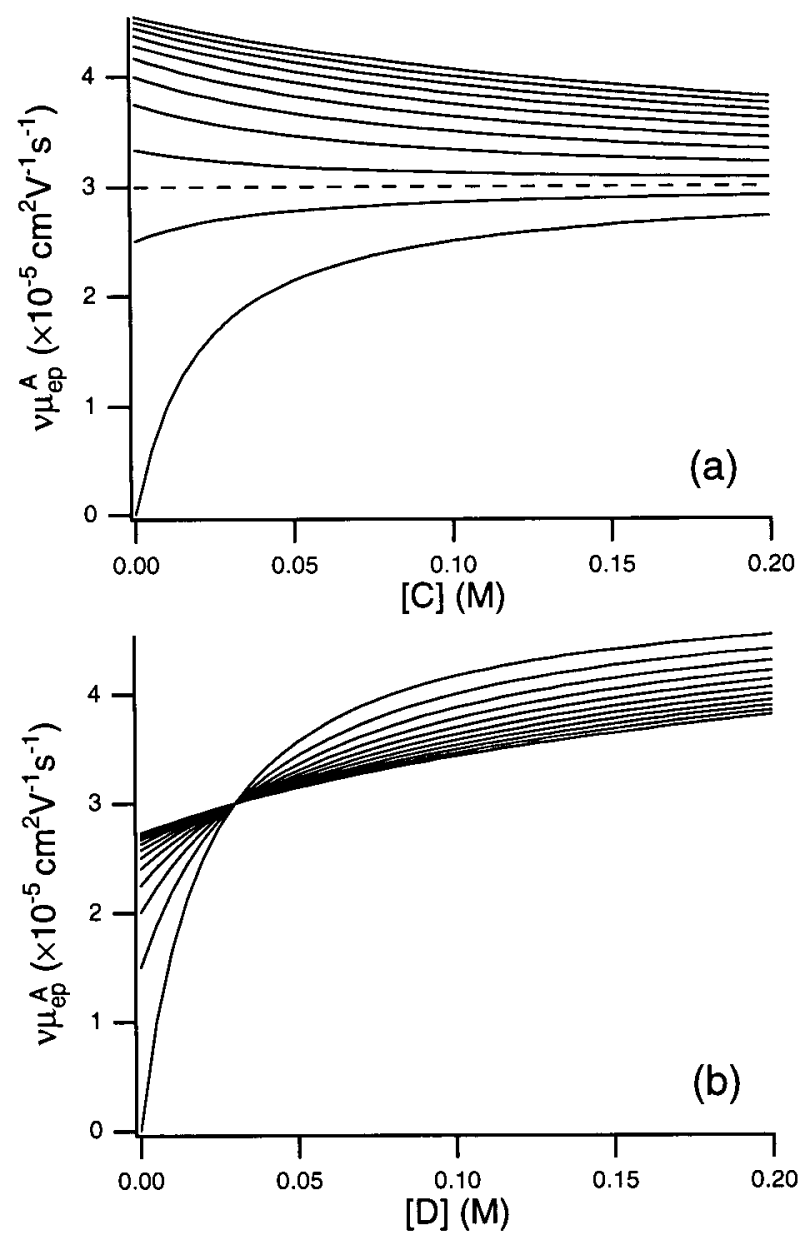

Figure 1.4.3 Profile plots of a binding isotherm surface where both additives interact with $1: 1$ binding stoichiometries (eq 1.4.1). The constants used to generate the curves are: $\mathrm{K}_{\mathrm{AC}}=50 \mathrm{M}^{-1} ; \mathrm{K}_{\mathrm{AD}}=50 \mathrm{M}^{-1} ; \mu_{\mathrm{ep}, \mathrm{A}}$ $=0 \mathrm{~cm}^{2} \mathrm{~V}^{-1} \mathrm{~s}^{-1} ; \mu_{\mathrm{op}, \mathrm{AC}}=3 \times 10^{-5} \mathrm{~cm}^{2} \mathrm{~V}^{-1} \mathrm{~s}^{-1} ; \mu_{\mathrm{op}, \mathrm{AD}}=5 \times 10^{-5} \mathrm{~cm}^{2} \mathrm{~V}^{-1} \mathrm{~s}^{-1}$. The second additive concentration ranges from 0 to $0.2 \mathrm{M}$ in increments of $0.02 \mathrm{M}$. The dashed line in (a) is the binding isotherm for $C$ when [D] is at the dengsu concentration.

Figure 1.4.3(b) shows the profile of the surface perpendicular to the [D] axis. The dengsu point is where all of the isotherms intersect. At $[D]_{D}$, the net mobility of the analyte is the same at any concentration of $\mathrm{C}$. Therefore, any isotherm for $\mathrm{D}$ must intersect at the dengsu concentration, regardless of the concentration of $\mathrm{C}$. The net analyte mobility at the dengsu concentration (i.e. the intersection on the profile plots) is equal to $\mu_{\mathrm{ep.AC}}$. It should be noted that a dengsu point can be detected using profile plots regardless of the concentrations of $\mathrm{C}$ at which the binding isotherms are measured. If several binding isotherms for D, measured at different [C], have a common intersection point, then a dengsu point is present. 
When [D] is constant, as is the case in the individual isotherms in Figure 1.4.3(a), eq 1.4.1 can be simplified to:

$v \mu_{\mathrm{ep}}^{\mathrm{A}}=\frac{\mu_{\mathrm{ep}, \mathrm{A}} *+\mathrm{K}_{\mathrm{AC}} *[\mathrm{C}] \mu_{\mathrm{ep}, \mathrm{AC}}}{1+\mathrm{K}_{\mathrm{AC}} *[\mathrm{C}]}$

where the asterisks $(*)$ indicate the apparent parameters in the presence of constant [D]:

$$
\begin{aligned}
& \mu_{\mathrm{ep}, \mathrm{A}} *=\frac{\mu_{\mathrm{ep}, \mathrm{A}}+\mathrm{K}_{\mathrm{AD}}[\mathrm{D}] \mu_{\mathrm{ep}, \mathrm{AD}}}{1+\mathrm{K}_{\mathrm{AD}}[\mathrm{D}]} \\
& \mathrm{K}_{\mathrm{AC}} *=\frac{\mathrm{K}_{\mathrm{AC}}}{1+\mathrm{K}_{\mathrm{AD}}[\mathrm{D}]}
\end{aligned}
$$

Equation 1.4.5 takes the same form as the equation for a one additive system. It is therefore impossible to determine if a second equilibrium is taking place from the shape of the isotherm when the concentration of the second additive remains constant. The apparent free mobility $\left(\mu_{\mathrm{ep}, \mathrm{A}} *\right)$ is determined by the interaction between the analyte and $\mathrm{D}$, as shown in Figure 1.4.3, where $\mu_{\mathrm{cp}, \mathrm{A}}{ }^{*}$ increases with the concentration of D. It is this shift in the apparent free mobility that gives rise to the dengsu point. When [D] is at the dengsu concentration, $\mu_{\mathrm{ep}, \mathrm{A}} *$ is equal to $\mu_{\mathrm{ep}, \mathrm{AC}}$. Substituting $\mu_{\mathrm{ep}, \mathrm{Ac}}$ for $\mu_{\mathrm{ep}, \mathrm{A}} *$ in eq 1.4 .5 gives: $v \mu_{\mathrm{ep}}^{\mathrm{A}}=\mu_{\mathrm{ep}, \mathrm{AC}}$. Since the apparent free mobility is equal to the complex mobility at the dengsu point, formation of the complex AC does not affect the net analyte mobility.

As binding with $\mathrm{D}$ increases, the apparent equilibrium constant for the binding of $\mathrm{A}$ and $\mathrm{C}\left(\mathrm{K}_{\mathrm{AC}}{ }^{*}\right)$ decreases. This can be seen clearly in Figure 1.4.2(a), where the curvature of the isotherms decreases as the concentration of $D$ increases. Changing $[D]$ is analogous to changing the environment in which the analyte interacts with C. It is important to remember that the constants measured in a binding experiment depend heavily on the conditions under which they are measured. It is impossible in CE, and most other methods, to study an equilibrium 
independently of other interactions. In CE, there are always interactions between the analyte and the solvent, buffer, counter ions or other compounds in solution. When the experimental conditions are changed, such as a change in the concentration of $\mathrm{D}$ or the addition of an organic modifier, a new set of apparent constants are often measured without considering the relationship between the two sets of conditions. Instead of empirically measuring apparent constants for different $\mathrm{D}$ concentrations, the interaction between the analyte and $\mathrm{D}$ can be considered as a second equilibrium, allowing a more systematic description of the system. The effect of solvent and buffer interactions on the apparent constants are often similar to changing the concentration of D. Generally speaking, if the interactions between an analyte and the solvent increase, the apparent equilibrium constant between the analyte and the additive will decrease ${ }^{(66,73)}$. This solvation effect can be thought of as a secondary equilibrium which competes with the analyteadditive interaction. It should be possible to describe analyte-solvent and analyte-buffer interactions in terms of equilibria, providing a better understanding of how these parameters affect analyte mobility.

The presence of an unknown secondary interaction, such as an analyte-solvent or analytebuffer interaction, can have important consequences in the application of single additive CE experiments. Although the shape of the binding isotherm can still be described using eq 1.4.5, secondary equilibria may cause results that seem puzzling. This can be demonstrated using the profile plots in Figure 1.4.3. If the analyte is involved in a secondary interaction with $\mathrm{D}$, the binding isotherm for $\mathrm{C}$ could take the shape of any of the curves shown in Figure 1.4.3(a), depending on the experimental conditions. In some cases, $\mathrm{C}$ would cause an increase in the net analyte mobility and in others a decrease. If $[D]$ is at the dengsu concentration, it will appear that $\mathrm{K}_{\mathrm{AC}}$ is zero even if binding does occur. Secondary equilibria need to be considered to systematically describe an analyte-additive interaction. 


\subsubsection{Higher Order Interactions}

As shown in chapter 1.3, not all analyte-additive interactions in CE take place with a 1:1 stoichiometry. It is therefore important to consider the effect of higher order interactions on binding isotherms when more than one additive is used. The simplest case to consider is when one additive (D) interacts with the analyte with a 1:1 stoichiometry and another additive (C) interacts with both 1:1 and 1:2 stoichiometries. The equation that describes the net mobility of the analyte can be determined using the general equation presented in chapter 1.2 (eq 1.2.11):

$$
v \mu_{e p}^{A}=\frac{\sum_{i=1}^{m} k_{i}^{\prime} \mu_{e p, i}}{\sum_{i=1}^{m} k_{i}^{\prime}}
$$

There will be four species of the analyte in this system: the uncomplexed analyte (A), the first order complex with $\mathrm{C}$, the second order complex with $\mathrm{C}$, and the first order complex with $\mathrm{D}$. Therefore, the net analyte mobility is given by:

$$
\begin{aligned}
& v \mu_{\mathrm{ep}}^{\mathrm{A}}=\frac{k_{\mathrm{A}}^{\prime} \mu_{\mathrm{ep}, \mathrm{A}}+k_{\mathrm{AC}}^{\prime} \mu_{\mathrm{ep}, \mathrm{AC}}+k_{\mathrm{AC}}^{\prime} \mu_{\mathrm{ep}, \mathrm{AC}}+k_{\mathrm{AD}}^{\prime} \mu_{\mathrm{ep}, \mathrm{AD}}}{k_{\mathrm{A}}^{\prime}+k_{\mathrm{AC}}^{\prime}+k_{\mathrm{AC}}^{\prime}+k_{\mathrm{AD}}^{\prime}} \\
& v \mu_{\mathrm{ep}}^{\mathrm{A}}=\frac{\mu_{\mathrm{ep}, \mathrm{A}}+\mathrm{K}_{\mathrm{AC}}^{\prime}[\mathrm{C}] \mu_{\mathrm{ep}, \mathrm{AC}}+\mathrm{K}_{\mathrm{AC}} \mathrm{K}_{\mathrm{AC}}[\mathrm{C}]^{2} \mu_{\mathrm{ep}, \mathrm{AC}}+\mathrm{K}_{\mathrm{AD}}[\mathrm{D}] \mu_{\mathrm{ep}, \mathrm{AD}}}{1+\mathrm{K}_{\mathrm{AC}}[\mathrm{C}]+\mathrm{K}_{\mathrm{AC}} \mathrm{K}_{\mathrm{AC}}[\mathrm{C}]^{2}+\mathrm{K}_{\mathrm{AD}}[\mathrm{D}]}
\end{aligned}
$$

Figure 1.4.4 shows the contour plot of an isotherm surface described by eq 1.4.10. The slopes of the contour curves are equal to the partial derivative of [D] with respect to [C]:

$$
\frac{\partial[D]}{\partial[C]}=-\frac{K_{A C}\left(v \mu_{e p}^{\mathrm{A}}-\mu_{e p, A C}\right)+2 K_{A C} K_{A C_{2}}[C]\left(v \mu_{e p}^{\mathrm{A}}-\mu_{e p, A C_{2}}\right)}{K_{A D}\left(v \mu_{e p}^{\mathrm{A}}-\mu_{e p, A D}\right)}
$$

The slopes of the contour curves are now dependent on the concentration of $\mathrm{C}$, indicating that the contour curves are generally not straight lines. The only case where the contour curve will be a straight line is when $v \mu_{\mathrm{ep}}^{\mathrm{A}}$ is equal to $\mu_{\mathrm{ep}, \mathrm{AC}}$ or $\mu_{\mathrm{ep}, \mathrm{AD}}$. For a dengsu point to exist, there must be a 
linear contour curve perpendicular to one of the concentration axes. If the slope of the contour curve is zero, there will be a dengsu concentration for $\mathrm{D}$; if the slope is infinity, there will be a dengsu concentration for $\mathrm{C}$.

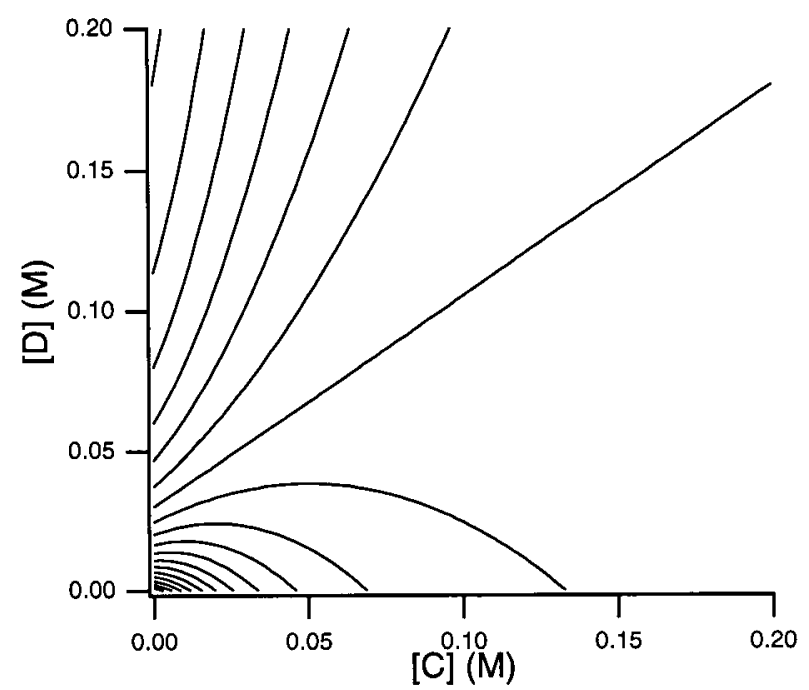

Figure 1.4.4 Contour plot of a binding isotherm surface where additive $C$ interacts with both $1: 1$ and $1: 2$ stoichiometries and additive $D$ has a $1: 1$ binding stoichiometry (eq 1.4.10). The constants are: $\mathrm{K}_{\mathrm{AC}}=50 \mathrm{M}^{-1} ; \mathrm{K}_{\mathrm{AC}_{2}}=$ $50 \mathrm{M}^{-1} ; \mathrm{K}_{\mathrm{AD}}=50 \mathrm{M}^{-1} ; \mu_{\mathrm{ep}, \mathrm{A}}=0 \mathrm{~cm}^{2} \mathrm{~V}^{-1} \mathrm{~s}^{-1} ; \mu_{\mathrm{ep}, \mathrm{AC}}=1.5 \times 10^{-5} \mathrm{~cm}^{2} \mathrm{~V}^{-1} \mathrm{~s}^{-1} ; \mu_{\mathrm{ep}, A C_{2}}=$ $3 \times 10^{-5} \mathrm{~cm}^{2} V^{-1} \mathrm{~s}^{-1} ; \mu_{\text {ep,AD }}=5 \times 10^{-5} \mathrm{~cm}^{2} \mathrm{~V}^{-1} \mathrm{~s}^{-1}$. The contour curves range from $v \mu_{\mathrm{ep}}^{A}=0 \mathrm{~cm}^{2} V^{-1} \mathrm{~s}^{-1}$ to $4.5 \times 10^{-5} \mathrm{~cm}^{2} \mathrm{~V}^{-1} \mathrm{~s}^{-1}$ in increments of $0.25 \times 10^{-5} \mathrm{~cm}^{2} \mathrm{~V}^{-1} \mathrm{~s}^{-1}$ between the contour lines.

There will be a dengsu concentration for $\mathrm{C}$ whenever $v \mu_{\mathrm{ep}}^{\mathrm{A}}$ is equal to $\mu_{\mathrm{ep}, \mathrm{AD}}$, which is possible if $\left|\mu_{\mathrm{ep}, \mathrm{AC}}-\mu_{\mathrm{ep}, \mathrm{A}}\right|$ or $\left|\mu_{\mathrm{ep}, \mathrm{AC}}-\mu_{\mathrm{ep}, \mathrm{A}}\right|$ is greater than $\left|\mu_{\mathrm{ep}, \mathrm{AD}}-\mu_{\mathrm{ep}, \mathrm{A}}\right|$, and both additives shift the net mobility of the analyte in the same direction. Figure 1.4 .5 shows profile plots made using eq 1.4.10 when $\left|\mu_{\mathrm{ep}, \mathrm{AC}}-\mu_{\mathrm{ep}, \mathrm{A}}\right|$ is greater than $\left|\mu_{\mathrm{ep}, \mathrm{AD}}-\mu_{\mathrm{cp}, \mathrm{A}}\right|$. 
60.
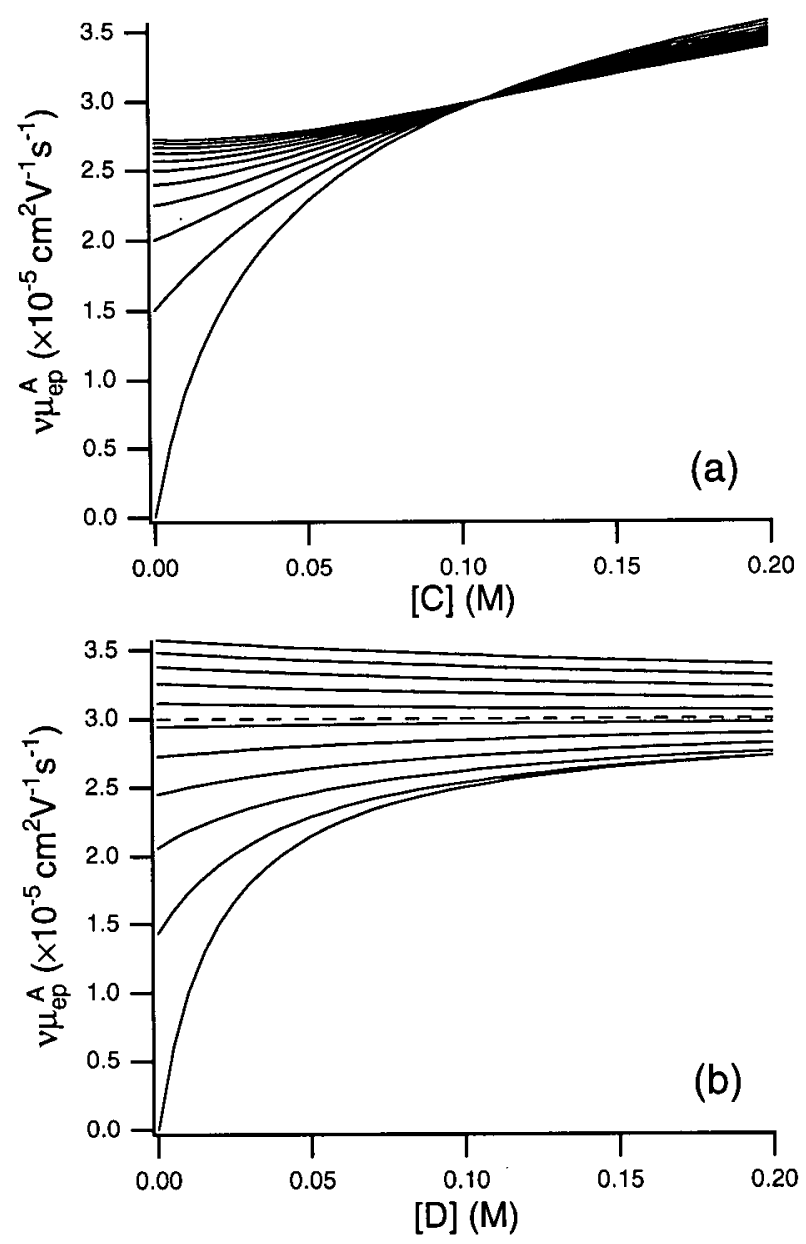

Figure 1.4.5 Profile plots of a binding isotherm surface where additive $C$ interacts with both $1: 1$ and $1: 2$ stoichiometries and additive $D$ interacts with a 1:1 binding stoichiometry (eq 1.4.11). The constants are: $K_{A C}=50$ $\mathrm{M}^{-1} ; \mathrm{K}_{\mathrm{AC}}=50 \mathrm{M}^{-1} ; \mathrm{K}_{\mathrm{AD}}=50 \mathrm{M}^{-1} ; \mu_{\mathrm{gP}, \mathrm{A}}=0 \mathrm{~cm}^{2} \mathrm{~V}^{-1} \mathrm{~s}^{-1} ; \mu_{\mathrm{AP}, \mathrm{AC}}=2.5 \times 10^{-5} \mathrm{~cm}^{2} \mathrm{~V}^{-1} \mathrm{~s}^{-1} ;$ $\mu_{\mathrm{op}, \mathrm{AC}}=5 \times 10^{-5} \mathrm{~cm}^{2} \mathrm{~V}^{-1} \mathrm{~s}^{-1} ; \mu_{\mathrm{ep}, \mathrm{AD}}=3 \times 10^{-5} \mathrm{~cm}^{2} \mathrm{~V}^{-1} \mathrm{~s}^{-1}$. The second additive concentration ranges from $0 \mathrm{M}$ to $0.2 \mathrm{M}$ in increments of $0.02 \mathrm{M}$. The dashed line in (b) is the binding isotherm for $D$ when $[C]$ is at the dengsu concentration.

The binding isotherms in Figure 1.4.5(a) have a common intersection point, indicating that there is a dengsu concentration for $\mathrm{C}$. The dengsu concentration can be determined by setting the partial derivative of $v \mu_{\mathrm{ep}}^{\mathrm{A}}$ with respect to [D] to zero, and solving for [C]:

$$
\frac{\partial v \mu_{e p}^{A}}{\partial[D]}=\frac{K_{A D}\left(\mu_{e p, A D}-\mu_{e p, A}\right)+K_{A C} K_{A D}[C]\left(\mu_{e p, A D}-\mu_{e p, A C}\right)+K_{A C} K_{A C} K_{A D}[C]^{2}\left(\mu_{e p, A D}-\mu_{e p, A C_{2}}\right)}{\left(1+K_{A C}[C]+K_{A C} K_{A C_{2}}[C]^{2}+K_{A D}[D]\right)^{2}}
$$


$0=K_{A D}\left(\mu_{e p, A D}-\mu_{e p, A}\right)+K_{A C} K_{A D}[C]_{D}\left(\mu_{e p, A D}-\mu_{e p, A C}\right)+K_{A C} K_{A C_{2}} K_{A D}[C]_{D}^{2}\left(\mu_{e p, A D}-\mu_{e p, A C_{2}}\right)$

The dengsu concentration for $\mathrm{C}$ (i.e. $[\mathrm{C}]_{\mathrm{D}}$ ) can be obtained by solving this quadratic equation (eq 1.4.13). The positive solutions correspond to the concentration of $\mathrm{C}$, where the net mobility is equal to $\mu_{e p, A D}$. If $\left|\mu_{e p, A C}-\mu_{e p, A}\right|>\left|\mu_{e p, A D}-\mu_{e p, A}\right|>\left|\mu_{e p, A C}-\mu_{e p, A}\right|$, there will be two positive solutions, and therefore two dengsu points. This will be very uncommon in CE because $\left|\mu_{\mathrm{ep}, \mathrm{AC}}-\mu_{\mathrm{ep}, \mathrm{A}}\right|$ is generally larger than $\mid \mu_{\mathrm{ep}, \mathrm{AC}}-\mu_{\mathrm{ep}, \mathrm{A}} \mathrm{l}$
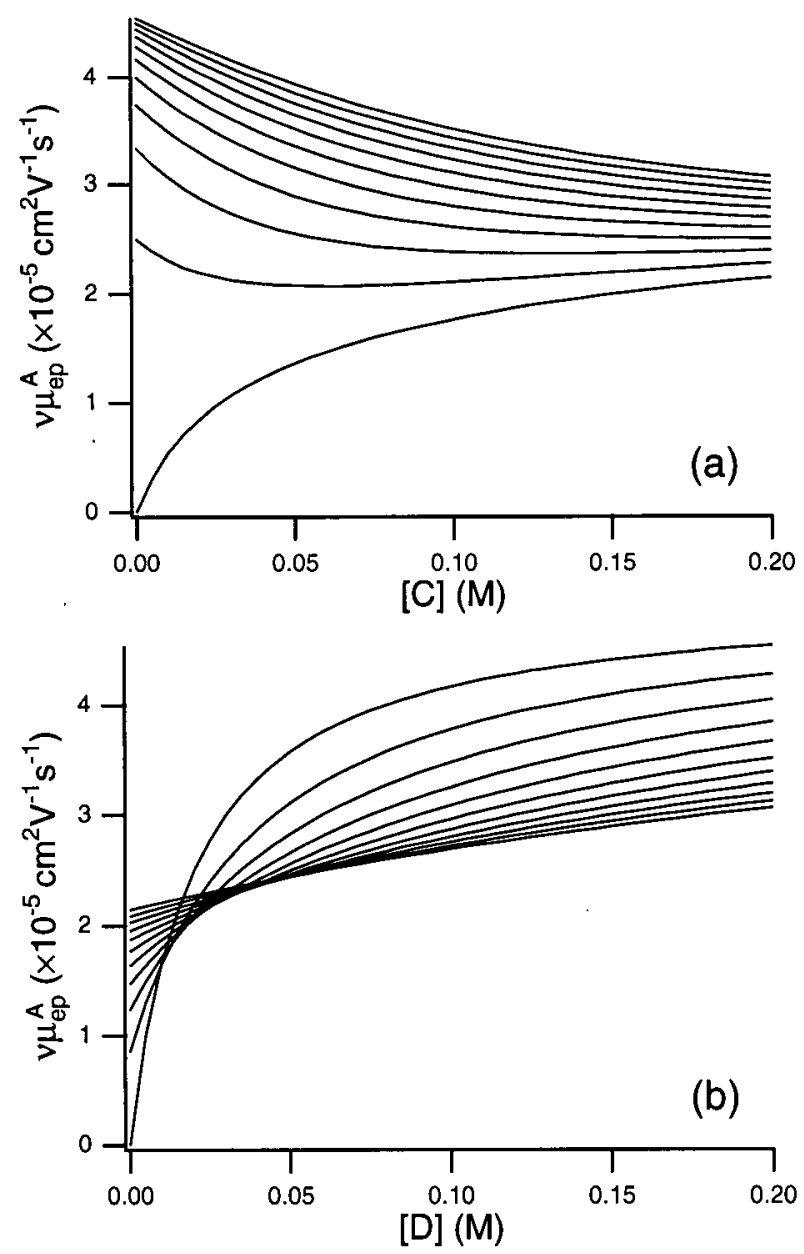

Figure 1.4.6 Profile plots of a binding isotherm surface where additive $C$ interacts with both $1: 1$ and $1: 2$ stoichiometries and additive $D$ interacts with a 1:1 binding stoichiometry (eq 1.4.10). The constants are: $K_{A C}=50$ $\mathrm{M}^{-1} ; \mathrm{K}_{\mathrm{AC}}=50 \mathrm{M}^{-1} ; \mathrm{K}_{\mathrm{AD}}=50 \mathrm{M}^{-1} ; \mu_{\mathrm{ep}, \mathrm{A}}=0 \mathrm{~cm}^{2} \mathrm{~V}^{-1} \mathrm{~s}^{-1} ; \mu_{\mathrm{ep}, \mathrm{AC}}=1.5 \times 10^{-5} \mathrm{~cm}^{2} \mathrm{~V}^{-1} \mathrm{~s}^{-1} ;$ $\mu_{\mathrm{op}, \mathrm{AC} \mathrm{C}_{2}}=3 \times 10^{-5} \mathrm{~cm}^{2} \mathrm{~V}^{-1} \mathrm{~s}^{-1} ; \mu_{\mathrm{op}, \mathrm{AD}}=5 \times 10^{-5} \mathrm{~cm}^{2} \mathrm{~V}^{-1} \mathrm{~s}^{-1}$. The second additive concentration ranges from $0 \mathrm{M}$ to $0.2 \mathrm{M}$ at increments of $0.02 \mathrm{M}$. 
Figure 1.4.6 shows profile plots made using eq 1.4.10 when $\left|\mu_{\text {ep,AD }}-\mu_{\text {ep, } A}\right|>\left|\mu_{\text {cp, Ac }}-\mu_{\text {ep, } A}\right|$.

The isotherms intersect at different concentrations of D (see Figure 1.4.6(b)), indicating that there is no dengsu point. In most situations where higher order interactions take place with one additive, usually there will be no dengsu concentration for the second additive. There are several exceptions when a dengsu concentration for D will exist, even when there are higher order interactions between the analyte and $\mathrm{C}$.

The most obvious case is when $\mathrm{K}_{\mathrm{AC}}$ is zero. No higher order interactions take place, and therefore, the conditions for the presence of a dengsu point are identical to those for a system with only $1: 1$ binding.

The second case is when $\mathrm{K}_{\mathrm{AC}}$ is zero, as demonstrated in Figure 1.4.7. This corresponds to strongly cooperative binding, where complexation of the second additive is more favorable than the first ${ }^{(66,73)}$. Steric effects usually make cooperative binding relatively uncommon for small molecules. However, cooperative binding is more common in interactions involving larger biological molecules. Cases where $\mathrm{C}$ does interact with the analyte cooperatively are easily identified using profile plots. The sigmoidal-shape of the binding isotherm at $[D]=0$ in Figure 1.4.7(a) indicates that cooperative binding is taking place ${ }^{(66,73)}$. Cases when cooperative binding causes a dengsu point for one additive, even though higher order interactions take place with the second additive, are easily identified by this distinctive sigmoidal-shaped isotherm in the profile plot.

The third case for the presence of a dengsu concentration for $\mathrm{D}$, when $\mathrm{C}$ undergoes higher order interactions with the analyte, is when $\mu_{e p, A C}=\mu_{e p, A C}=v \mu_{c p}^{A}$. This case is extremely uncommon in CE. The purpose of using complexation additives is to modify the net mobility of an analyte. It is difficult to imagine any case where the binding of one additive changes the mobility of an analyte but the mobility does not change with further complexation. 
63.
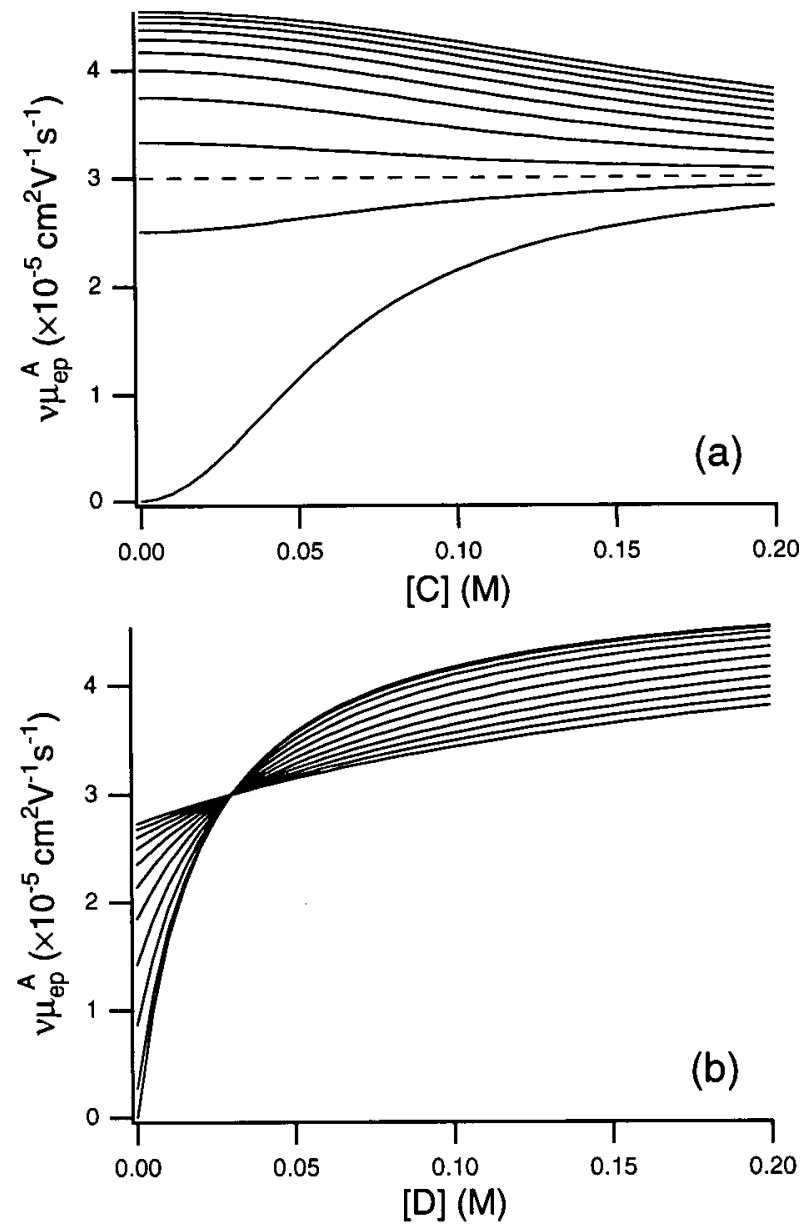

Figure 1.4.7 Profile plots of a binding isotherm surface where additive $C$ interacts with a 1:2 stoichiometry and additive $D$ has a $1: 1$ binding stoichiometry (eq. 1.4.10). The constants are: $\mathrm{K}_{\mathrm{AC}}=0 \mathrm{M}^{-1} ; \mathrm{K}_{\mathrm{AC}} \mathrm{K}_{\mathrm{AC}_{2}}=250$ $M^{-2} ; K_{A D}=50 M^{-1} ; \mu_{e p, A}=0 \mathrm{~cm}^{2} V^{-1} \mathrm{~s}^{-1} ; \mu_{\text {ep }, A C_{2}}=3 \times 10^{-5} \mathrm{~cm}^{2} V^{-1} \mathrm{~s}^{-1} ; \mu_{\text {ep }, A D}=5 \times 10^{-5}$ $\mathrm{cm}^{2} \mathrm{~V}^{-1} \mathrm{~s}^{-1}$. The second additive concentration ranges from $0 \mathrm{M}$ to $0.2 \mathrm{M}$ with $0.02 \mathrm{M}$ increments between the profile lines. The dashed line in (a) is where the concentration of $D$ is at the dengsu concentration.

In the exceptions when there is a dengsu concentration for $\mathrm{D}$ when $\mathrm{C}$ undergoes higher order interactions with the analyte, $[D]_{D}$ can be determined by setting the partial derivative of $v \mu_{\mathrm{ep}}^{\mathrm{A}}$ with respect to [C] to zero and solving for [D]:

$$
\frac{\partial v \mu_{\mathrm{ep}}^{\mathrm{A}}}{\partial[\mathrm{D}]}=\frac{\begin{array}{l}
\mathrm{K}_{\mathrm{AC}}\left(\mu_{\mathrm{ep}, \mathrm{AC}}-\mu_{\mathrm{ep}, \mathrm{A}}\right)+\mathrm{K}_{\mathrm{AC}}{ }^{2} \mathrm{~K}_{\mathrm{AC}}[\mathrm{C}]^{2}\left(\mu_{\mathrm{ep}, \mathrm{AC}}-\mu_{\mathrm{ep}, \mathrm{AC}}\right)+\mathrm{K}_{\mathrm{AC}} \mathrm{K}_{\mathrm{AD}}[\mathrm{D}]\left(\mu_{\mathrm{ep}, \mathrm{AC}}-\mu_{\mathrm{ep}, \mathrm{AD}}\right) \\
+2 \mathrm{~K}_{\mathrm{AC}}[\mathrm{C}]\left(\mu_{\mathrm{ep}, \mathrm{AC}}-\mu_{\mathrm{ep}, \mathrm{A}}\right)+2 \mathrm{~K}_{\mathrm{AC}} \mathrm{K}_{\mathrm{AC}} \mathrm{K}_{\mathrm{AD}}[\mathrm{C}][\mathrm{D}]\left(\mu_{\mathrm{ep}, \mathrm{AC}}-\mu_{\mathrm{ep}, \mathrm{AD}}\right)
\end{array}}{\left(1+\mathrm{K}_{\mathrm{AC}}[\mathrm{C}]+\mathrm{K}_{\mathrm{AC}} \mathrm{K}_{\mathrm{AC}_{2}}[\mathrm{C}]^{2}+\mathrm{K}_{\mathrm{AD}}[\mathrm{D}]\right)^{2}}
$$


64.

When $\mathrm{K}_{\mathrm{AC}}$ is zero (highly cooperative $1: 2$ interaction) or $\mu_{\mathrm{ep}, \mathrm{AC}}=\mu_{\mathrm{ep}, \mathrm{AC}}$, setting eq 1.4.14 to zero gives:

$[D]_{D}=\frac{\left(\mu_{e p, A}-\mu_{e p, A C_{2}}\right)}{K_{A D}\left(\mu_{e p, A C_{2}}-\mu_{e p, A D}\right)}$

which is identical to eq 1.4 .4 .

As stated earlier, it is uncommon for a dengsu concentration to exist when the second additive undergoes higher order interactions with the analyte. The exceptions to these cases are either easily identified or rarely occur. Therefore, the presence of a dengsu concentration for one additive is a strong indication that the second additive interacts with the analyte in a $1: 1$ stoichiometry over the range of additive concentrations tested. In practical applications, a dengsu point can be used as a diagnostic tool to determine if higher order interactions need to be considered to describe a particular analyte-additive interaction. Several binding isotherms for the interaction between the analyte and a second additive can be measured at different concentrations of the additive for which the binding stoichiometry is to be determined. If the isotherms share a common intersection point and the second additive does not undergo cooperative binding with the analyte, then the additive binds the analyte with a $1: 1$ stoichiometry. It should be emphasized that this does not exclude higher order interactions taking place at higher additive concentrations (see section 1.3.5). If the binding isotherms intersect but there is no dengsu point, it is almost certain that more complicated interactions are taking place.

Although only one additive can have a dengsu concentration, the profile plots of two additive systems allow the unambiguous determination of the binding stoichiometry for both additives. As in the case where both additives interact with a 1:1 stoichiometry, the isotherms in the profile plot are drawn by keeping the concentration of the second additive constant. If [C] is 
constant, eq 1.4.10 can be simplified to:

$v \mu_{\mathrm{ep}}^{\mathrm{A}}=\frac{\mu_{\mathrm{ep}, \mathrm{A}} *+\mathrm{K}_{\mathrm{AD}} *[\mathrm{D}] \mu_{\mathrm{ep}, \mathrm{AD}}}{1+\mathrm{K}_{\mathrm{AD}} *[\mathrm{D}]}$

where:

$\mu_{\mathrm{ep}, \mathrm{A}} *=\frac{\mu_{\mathrm{ep}, \mathrm{A}}+\mathrm{K}_{\mathrm{AC}}[\mathrm{C}] \mu_{\mathrm{ep}, \mathrm{AC}}+\mathrm{K}_{\mathrm{AC}} \mathrm{K}_{\mathrm{AC}}[\mathrm{C}]^{2} \mu_{\mathrm{ep}, \mathrm{AC}}}{1+\mathrm{K}_{\mathrm{AC}}[\mathrm{C}]+\mathrm{K}_{\mathrm{AC}} \mathrm{K}_{\mathrm{AC}_{2}}[\mathrm{C}]^{2}}$

$\mathrm{K}_{\mathrm{AD}} *=\frac{\mathrm{K}_{\mathrm{AD}}}{1+\mathrm{K}_{\mathrm{AC}}[\mathrm{C}]+\mathrm{K}_{\mathrm{AC}} \mathrm{K}_{\mathrm{AC}_{2}}[\mathrm{C}]^{2}}$

Even though the analyte undergoes higher order interactions with $C$, when $[C]$ is constant, the binding isotherms for $\mathrm{D}$ can be described using the equation for a first order, one additive system, with apparent constants given by eqs 1.4 .17 and 1.4.18.

The isotherms for $\mathrm{C}$ keeping [D] constant are described by the equation:

$v \mu_{e p}^{\mathrm{A}}=\frac{\mu_{\mathrm{ep}, \mathrm{A}} *+\mathrm{K}_{\mathrm{AC}} *[\mathrm{C}] \mu_{\mathrm{ep}, \mathrm{AC}}+\mathrm{K}_{\mathrm{AC}} * \mathrm{~K}_{\mathrm{AC}}[\mathrm{C}]^{2} \mu_{\mathrm{ep}, \mathrm{AC}}}{1+\mathrm{K}_{\mathrm{AC}} *[\mathrm{C}]+\mathrm{K}_{\mathrm{AC}} * \mathrm{~K}_{\mathrm{AC}_{2}}[\mathrm{C}]^{2}}$

where:

$\mu_{\mathrm{ep}, \mathrm{A}} *=\frac{\mu_{\mathrm{ep}, \mathrm{A}}+\mathrm{K}_{\mathrm{AD}}[\mathrm{D}] \mu_{\mathrm{ep}, \mathrm{AD}}}{1+\mathrm{K}_{\mathrm{AD}}[\mathrm{D}]}$

$\mathrm{K}_{\mathrm{AC}} *=\frac{\mathrm{K}_{\mathrm{AC}}}{1+\mathrm{K}_{\mathrm{AD}}[\mathrm{D}]}$

The isotherms for $\mathrm{C}$ at constant [D] can be described using the equation for interactions with a single additive, with binding stoichiometries of $1: 1$ and 1:2. It is generally true, when the concentration of the second additive is kept constant, that the shape of the isotherms for an additive is determined by the stoichiometry of the interactions between that additive and the analyte. The apparent constants in the equation describing the isotherms change with the 
concentration of the second additive.

A method for detecting the presence of higher order interactions was discussed in section 1.3.5. It was noted that it is impossible to detect higher order interactions when the microscopic equilibrium constants and complex mobilities are equal. For a system with multiple equilibria, it would be more accurate to say that it is impossible to detect higher order interactions in a binding isotherm when the apparent microscopic constants are equal. As shown by eq 1.4.19, the apparent equilibrium constants are dependent on the concentration of the second additive. If binding isotherms for $\mathrm{C}$ are measured at different $[\mathrm{D}]$, the apparent microscopic constants can not be equal in all cases. If higher order interactions are not detected in any of the isotherms, then binding occurs with a 1:1 stoichiometry. If higher order interactions take place, it will be noticeable in most, if not all, of the binding isotherms. This suggests that a second additive could be added to a one additive system to unambiguously determine the binding stoichiometry of the first additive.

The conditions for a dengsu point to occur when higher order interactions take place (i.e. $\mathrm{K}_{\mathrm{AC}}=0$ ) are very different from those when higher order interactions are not detectable in the binding isotherm (i.e. the microscopic constants are equal). If both methods are used in conjunction, higher order interactions can always be detected in at least one of the methods.

\subsubsection{Interactions Between Additives}

Implicit in the above discussion was the absence of interactions between the additives $\mathrm{C}$ and D. When this assumption is valid, the constants measured for each individual additive can be used in eq 1.4 .1 to predict the net mobility of the analyte for any combination of the two additives. The discussion in this section will demonstrate how the dengsu point and the binding isotherms in the profile plots can be used to determine if interactions between the additives need 
to be considered.

One of the common assumptions made in $\mathrm{CE}$ with additives is that the initial concentrations of the additives $\left([C]_{0}\right.$ and $\left.[D]_{0}\right)$ are not changed significantly during the dynamic complexation process. In systems where the additives only interact with the analyte, $[\mathrm{C}]_{0}$ and $[D]_{0}$ can be substituted for $[C]$ and $[D]$ if the concentrations of the additives are much larger than those of the analyte ([C] and [D] are the concentrations of uncomplexed additives after the system reaches equilibrium). If there is significant interaction between $\mathrm{C}$ and $\mathrm{D}$ then the above assumption may no longer be valid. If $\mathrm{C}$ and $\mathrm{D}$ interact with a 1:1 stoichiometry, [C] can be determined by solving the following quadratic equation:

$0=\mathrm{K}_{\mathrm{CD}}[\mathrm{C}]^{2}+\left(\mathrm{K}_{\mathrm{CD}}[\mathrm{C}]_{0}+\mathrm{K}_{\mathrm{CD}}[\mathrm{D}]_{0}+1\right)[\mathrm{C}]-[\mathrm{C}]_{0}$

where $\mathrm{K}_{\mathrm{CD}}$ is the formation constant for the complex $\mathrm{CD}$. A similar expression can be written for [D]. Therefore, $\mathrm{K}_{\mathrm{CD}}$ must be measured to calculate [C] and [D].

Substituting $[D]_{0}$ and $[C]_{0}$ for $[C]$ and $[D]$ when it is not valid to do so can have a major effect on the binding isotherms. This is true even if there is no interaction between the analyte and the complex formed by the two different additives (CD). If there is no interaction between the analyte and $\mathrm{CD}$, then the net mobility of the analyte can still be described using eq 1.4.1. Therefore, if the binding isotherms are plotted using $[\mathrm{C}]$ and $[\mathrm{D}]$, the profile plots will be identical to those shown in Figure 1.4.3.

Figure 1.4.8 shows profile plots made with $[C]_{0}$ and $[D]_{0}$ when $C$ and $D$ interact. Clearly, there is no dengsu point. Also, the shapes of the isotherms can not be described based on equilibria with a 1:1 stoichiometry. Therefore, the presence of a dengsu concentration for either additive indicates that there is no significant interaction between $\mathrm{C}$ and $\mathrm{D}$, and the assumptions that $[C]=[C]_{0}$ and $[D]=[D]_{0}$ are valid. 
68.
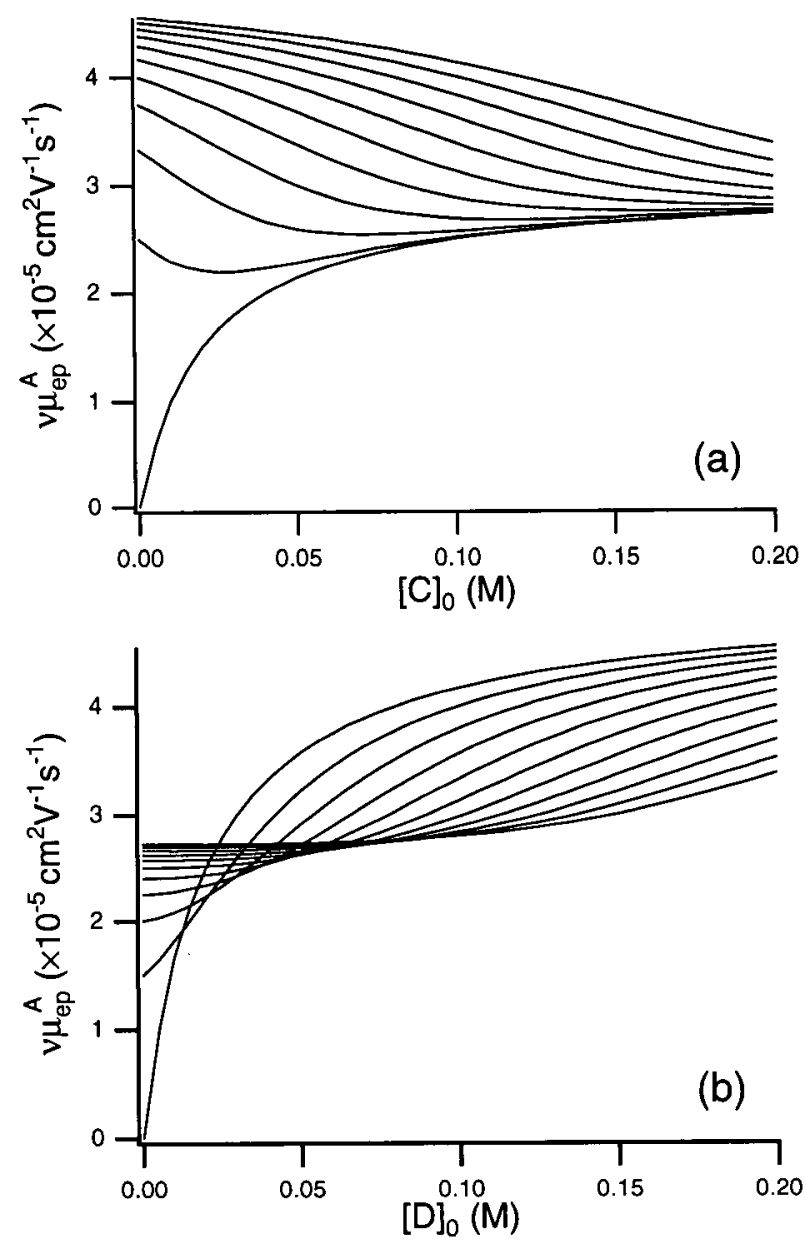

Figure 1.4.8 Profile piots of a binding isotherm surface where interactions between the additives invalidate the assumption $[C]=[C]_{0}$ and $[D]=[D]_{0}$ (eq 1.4.22). The constants are: $K_{A C}=50 \mathrm{M}^{-1} ; K_{A D}=50 \mathrm{M}^{-1}$; $\mathrm{K}_{\mathrm{CD}}=50 \mathrm{M}^{-1} ; \mathrm{K}_{\mathrm{ACD}}=0 \mathrm{M}^{-1} ; \mu_{\mathrm{ep}, \mathrm{A}}=0 \mathrm{~cm}^{2} \mathrm{~V}^{-1} \mathrm{~s}^{-1} ; \mu_{\mathrm{ep}, \mathrm{AC}}=3 \times 10^{-5} \mathrm{~cm}^{2} \mathrm{~V}^{-1} \mathrm{~s}^{-1} ; \mu_{\mathrm{op}, \mathrm{AD}}=$ $5 \times 10^{-5} \mathrm{~cm}^{2} V^{-1} \mathrm{~s}^{-1}$. The second additive concentration ranges from $0 \mathrm{M}$ to $0.2 \mathrm{M}$ with $0.02 \mathrm{M}$ increments between the profile lines.

Even when the assumptions that $[C]=[C]_{0}$ and $[D]=[D]_{0}$ are valid, if the analyte can bind both additives at the same time, the constants measured for each additive individually may not accurately describe the net analyte mobility when both additives are used. If the analyte can bind both additives at the same time (forming the complex ACD), the net mobility of the analyte can be described by the equation:

$$
v \mu_{\mathrm{ep}}^{\mathrm{A}}=\frac{k_{\mathrm{A}}^{\prime} \mu_{\mathrm{ep}, \mathrm{A}}+k_{\mathrm{AC}}^{\prime} \mu_{\mathrm{ep}, \mathrm{AC}}+k_{\mathrm{AD}}^{\prime} \mu_{\mathrm{ep}, \mathrm{AD}}+k_{\mathrm{ACD}}^{\prime} \mu_{\mathrm{ep}, \mathrm{ACD}}}{k_{\mathrm{A}}^{\prime}+k_{\mathrm{AC}}^{\prime}+k_{\mathrm{AD}}^{\prime}+k_{\mathrm{ACD}}^{\prime}}
$$


$v \mu_{\mathrm{ep}}^{\mathrm{A}}=\frac{\mu_{\mathrm{ep}, \mathrm{A}}+\mathrm{K}_{\mathrm{AC}}[\mathrm{C}] \mu_{\mathrm{ep}, \mathrm{AC}}+\mathrm{K}_{\mathrm{AD}}[\mathrm{D}] \mu_{\mathrm{ep}, \mathrm{AD}}+\mathrm{K}_{\mathrm{ACD}}[\mathrm{C}][\mathrm{D}] \mu_{\mathrm{ep}, \mathrm{ACD}}}{1+\mathrm{K}_{\mathrm{AC}}[\mathrm{C}]+\mathrm{K}_{\mathrm{AD}}[\mathrm{D}]+\mathrm{K}_{\mathrm{ACD}}[\mathrm{C}][\mathrm{D}]}$

where $\mathrm{K}_{\mathrm{ACD}}$ is the formation constant of the complex $\mathrm{ACD}$, and $\mu_{\mathrm{cp}, \mathrm{ACD}}$ is the mobility of the complex ACD.

The formation of a complex $\mathrm{ACD}$ can be detected using the isotherms of a profile plot. If [D] is kept constant, eq 1.4.24 can be simplified to:

$v \mu_{\mathrm{ep}}^{\mathrm{A}}=\frac{\mu_{\mathrm{ep}, \mathrm{A}} *+\mathrm{K}_{\mathrm{AC}} *[\mathrm{C}] \mu_{\mathrm{ep}, \mathrm{AC}} *}{1+\mathrm{K}_{\mathrm{AC}} *[\mathrm{C}]}$

where:

$\mu_{\mathrm{ep}, \mathrm{A}} *=\frac{\mu_{\mathrm{ep}, \mathrm{A}}+\mathrm{K}_{\mathrm{AD}}[\mathrm{D}] \mu_{\mathrm{ep}, \mathrm{AD}}}{1+\mathrm{K}_{\mathrm{AD}}[\mathrm{D}]}$

$\mathrm{K}_{\mathrm{AC}} *=\frac{\mathrm{K}_{\mathrm{AC}}+\mathrm{K}_{\mathrm{ACD}}[\mathrm{D}]}{1+\mathrm{K}_{\mathrm{AD}}[\mathrm{D}]}$

$\mu_{\mathrm{ep}, \mathrm{AC}} *=\frac{\mathrm{K}_{\mathrm{AC}} \mu_{\mathrm{ep}, \mathrm{AC}}+\mathrm{K}_{\mathrm{ACD}}[\mathrm{D}] \mu_{\mathrm{ep}, \mathrm{ACD}}}{\mathrm{K}_{\mathrm{AC}}+\mathrm{K}_{\mathrm{ACD}}[\mathrm{D}]}$

The major difference between eq 1.4.5 and eq 1.4.25 is the presence of an apparent complex mobility $\left(\mu_{\mathrm{ep}, \mathrm{Ac}}{ }^{*}\right)$ in eq 1.4.25. The apparent complex mobility for a particular isotherm is now dependent on [D]. Binding isotherms for $\mathrm{C}$ at different [D] will give different values for $\mu_{\mathrm{cp}, \mathrm{AC}}{ }^{*}$, unless $\mu_{\mathrm{ep}, A \mathrm{AC}}$ is equal to $\mu_{\mathrm{ep}, A C D}$. It will be uncommon for $\mu_{\mathrm{ep}, \mathrm{AC}}$ to be equal to $\mu_{\mathrm{ep}, A \mathrm{ACD}}$, since additives are chosen to modify the mobility of the analyte. Attaching a second additive to a singly bound analyte will almost always change the mobility of the analyte. The dependence of $\mu_{\mathrm{cp}, A c}{ }^{*}$ on [D] can be used to detect cases where the analyte can interact with both additives at the same time. If the apparent complex mobility remains constant at different [D], then the effect of complex ACD on the net mobility of the analyte does not need to be considered. If the apparent complex 
mobility changes at different [D], this is a strong indication that interactions between the additives need to be considered, and constants obtained using the individual additives may not accurately describe the mobility of the analyte when the additives are used together.

\subsubsection{Conclusions}

This chapter presented some of the unique properties of multivariate binding isotherms when two additives are used in CE. The dengsu concentration, when present, can be easily identified by a common intersection point in the profile plot. The presence of a dengsu point is a strong indication that the second additive interacts with the analyte with a 1:1 stoichiometry and there is no significant interaction between the additives. The shapes of the binding isotherms in the profile plots can be used to further confirm the binding stoichiometries of the two additives, as well as to determine whether the analyte can bind with both additives at the same time.

Although the discussion has been made in the context of a two additive system, many of the points made in this chapter are relevant to systems that employ a single additive, or systems with more than two additives. We have shown how a second additive can be used to unambiguously determine the stoichiometry of a particular analyte-additive interaction. It is necessary to understand the effect of a second additive on a binding isotherm even if the concentration of the second additive remains constant. Buffer ions, solvents, or other compounds present in the $\mathrm{CE}$ system may interact with the analyte. Although their concentrations may be constant if experimental conditions are kept the same, secondary equilibria may cause misleading results when studying an analyte-additive interaction if the possibility of more complicated equilibria are not considered. It is also important to remember that equilibrium constants are generally not measured independently of other interactions. Many secondary interactions are currently accounted for by reporting apparent constants for specific experimental conditions. In the future, it will be important to determine what phenomena are 
better described systematically using multiple equilibria, rather than relying on apparent constants measured for a specific set of experimental conditions. 


\subsection{The Effect of Complexation Additives on Analyte Migration Behavior in Capillary Electrochromatography}

\subsubsection{Introduction}

Electroosmosis was first suggested as an alternative "pumping" mechanism to hydrodynamic pressure in liquid chromatography and capillary action in thin layer chromatography by Pretorius et al.(15) in 1974 . Jorgenson and Lukas ${ }^{(9)}$ showed that electrochromatography can be performed in capillary columns in 1981. Most of the discussions on capillary electrochromatography (CEC) since then have focused on the relatively high efficiency of the separations ${ }^{(8,15,80-84)}$. The high efficiency is due to the flat flow profile of the $\operatorname{EOF}^{(15,16,83,84)}$. Increased number of theoretical plates can be achieved using stationary phases with relatively small particle sizes because the EOF is not affected by the particle size $^{(15,16,83,84)}$. The theoretical aspects of zone migration in electrochromatography, based on the mass balance equation (described in section 1.1.2), has been discussed recently(25).

Less attention has been paid to the migration behavior of the analytes in CEC. Typically, migration rates in CEC are discussed using standard chromatography theory, replacing the mobile phase velocity with the electroosmotic mobility $\left(\mu_{\mathrm{e} 0}\right)^{(16,85)}$. This approach limits the discussion to neutral analytes because standard chromatography theory does not account for the possibility of analytes having different mobilities in the mobile phase. Knox et al. recognized this limitation and proposed that analyte migration behavior be discussed in terms of effective capacity factors ${ }^{(16)}$. While this allows the migration rate of the analyte to be compared to the EOF, the physical meaning of the effective capacity factor was not clear. The results of one experiment can not be compared to those of another experiment under different conditions. Also, it appears that by limiting the theoretical discussion of migration rates in CEC to neutral analytes, many researchers have limited the application of CEC to neutral analytes as well. A better understanding of the migration behavior of charged analytes may lead to the development 
of a wider range of applications using CEC.

Another problem in using conventional chromatography theory to describe CEC is the premise that the phase distribution is the only equilibrium taking place. If a second equilibrium is introduced, such as dynamic complexation between an analyte and a buffer additive, conventional chromatography theory only gives an overall capacity factor, and cannot predict the analyte migration rate for different additive concentrations.

Rathore and Horváth recently discussed the parallel relationship between chromatography and electrophoresis using the concept of virtual migration distances ${ }^{(29)}$. As stated in section 1.1.3, this approach allows similar equations to be written for each method, but the meaning of the parameters used in these equations are defined differently. The electrophoretic velocity factor, proposed as analogous to the capacity factor in chromatography, only relates the electrophoretic mobility to the electroosmotic mobility as opposed to actually describing the analyte migration behavior. Because this approach is based on the conventional definition of the capacity factor, it cannot be used in cases where more than one equilibrium is present (e.g. CEC with buffer additives).

A general theory that can be applied in all major areas of separation science such as chromatography, capillary electrophoresis (with or without additives), and ultracentrifugation was presented in chapter 1.2. The theory accounts for analytes with different mobilities in the mobile phase as well as any number or type of equilibria. The following section presents the theoretical discussion of CEC as another case in a unified separation science. Emphasis will be placed on two CEC systems that are not easily described using conventional chromatographic theory: the presence of charged analytes and the effect of buffer additives. 


\subsubsection{CEC in the Absence of EOF}

Equation 1.2.10 can be used to describe the migration rate of an analyte in any separation technique in the presence of any number type of equilibria (assuming the equilibria take place at a fast rate in relation to the overall separation time):

$$
\overline{\mathrm{U}}^{\mathrm{A}}=\frac{\sum_{\mathrm{i}=1}^{\mathrm{m}} k_{\mathrm{i}}^{\prime} \overline{\mathrm{U}}_{\mathrm{i}}}{\sum_{\mathrm{i}=1}^{\mathrm{m}} k_{\mathrm{i}}^{\prime}}
$$

Analyte migration behavior in CEC is usually described in terms of mobility. allowing eq 1.5.1 to be rewritten as:

$$
\mu^{\mathrm{A}}=\frac{\sum_{\mathrm{i}=1}^{\mathrm{m}} k_{\mathrm{i}}^{\prime} \mu_{\mathrm{i}}}{\sum_{\mathrm{i}=1}^{\mathrm{m}} k_{\mathrm{i}}^{\prime}}
$$

where $\mu^{A}$ is the net mobility of the analyte, and $\mu_{i}$ is the mobility of analyte species $i$. The mobilities of all species in the stationary phase can be approximated to zero.

In the simplest CEC systems where the only equilibrium is the phase distribution of the analyte between the buffer and the stationary phase, and there is no EOF, eq 1.5 .2 can be simplified to:

$$
\mu^{\mathrm{A}}=\mu_{\mathrm{ep}}^{\mathrm{A}}=\frac{1}{1+k_{\mathrm{A}, \mathrm{S}}^{\prime}} \mu_{\mathrm{A}, \mathrm{M}}
$$

where $\mu_{\mathrm{ep}}^{\mathrm{A}}$ is the net electrophoretic mobility of the analyte, $k_{\mathrm{A}, \mathrm{S}}^{\prime}$ is the capacity factor of the analyte in the stationary phase, and $\mu_{\mathrm{A}, \mathrm{M}}$ is the electrophoretic mobility of the analyte in the buffer. The effect of the EOF on $\mu^{\mathrm{A}}$ will be discussed in a later section. Because the value of $\mathrm{k}_{\mathrm{A}, \mathrm{S}}^{\prime}$ in eq 1.5.3 is independent of the separation technique used to measure it, the capacity factor 
of the analyte in the stationary phase can be determined using capillary liquid chromatography with the same capillary column.

\subsubsection{The Effect of Buffer Additives}

Dynamic complexation theory can be used to account for the migration behavior of analytes with the presence of additives such as cyclodextrins or micelles, as well as acid-base equilibria where the hydrogen ion is considered the complexation additive. In conventional chromatography theory, changing the mobile phase in any way (i.e. additive concentration, $\mathrm{pH}$ etc.) changes the capacity factor. Therefore, it provides little guidance in the prediction of the analyte migration behavior when the additive concentration or $\mathrm{pH}$ is changed.

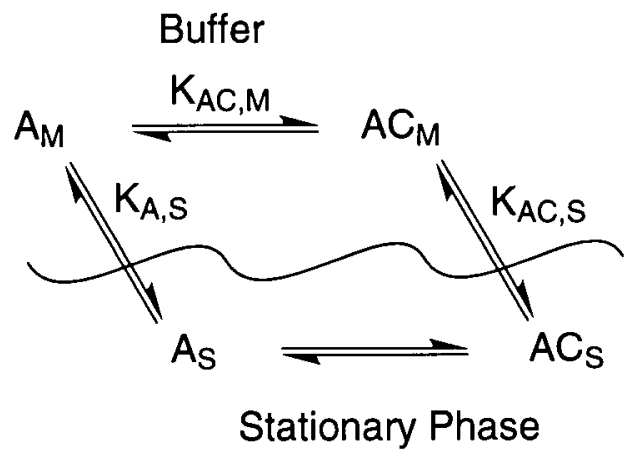

Figure 1.5.1 Equilibria describing CEC with a complexation additive present in the buffer. The analyte species present are: the free and complexed analyte $\left(A_{M}\right.$ and $\left.A_{M}\right)$ in the mobile phase and the uncomplexed and complexed analyte $\left(A_{s}\right.$ and $\left.A_{s}\right)$ in the stationary phase.

The individual capacity factors and the general equation describing the analyte migration behavior enable us to predict the effect of each individual equilibrium on the net analyte mobility in the separation system. Fig. 1.5.1 shows the equilibria that take place when an additive, which interacts with the analyte with a 1:1 stoichiometry, is present in the CEC buffer. There are four species of the analyte: the free analyte in the buffer $\left(A_{M}\right)$, the analyte-additive complex in the buffer $\left(\mathrm{AC}_{\mathrm{M}}\right)$, the uncomplexed analyte in the stationary phase $\left(\mathrm{A}_{\mathrm{S}}\right)$, and the analyte-additive complex in the stationary phase $\left(\mathrm{AC}_{\mathrm{s}}\right)$. The equation that describes the net mobility of the 
analyte in this system (in the absence of EOF) can be determined using eq 1.5.2:

$\mu_{\mathrm{ep}}^{\mathrm{A}}=\frac{k_{\mathrm{A}, \mathrm{M}}^{\prime} \mu_{\mathrm{A}, \mathrm{M}}+k_{\mathrm{AC}, \mathrm{M}}^{\prime} \mu_{\mathrm{AC}, \mathrm{M}}}{k_{\mathrm{A}, \mathrm{M}}^{\prime}+k_{\mathrm{AC}, \mathrm{M}}^{\prime}+k_{\mathrm{A}, \mathrm{S}}^{\prime}+k_{\mathrm{AC}, \mathrm{S}}^{\prime}}$

where $\mu_{\mathrm{A}, \mathrm{M}}$ and $\mu_{\mathrm{AC}, \mathrm{M}}$ are the electrophoretic mobilities of the free analyte and the analyte-additive complex in the buffer (i.e. mobile phase), $k_{\mathrm{A}, \mathrm{M}}^{\prime}$ and $k_{\mathrm{AC}, \mathrm{M}}^{\prime}$ are the capacity factors of the free analyte and the analyte-additive complex in the buffer, and $k_{\mathrm{A}, S}^{\prime}$ and $k_{\mathrm{AC}, \mathrm{S}}^{\prime}$ are the capacity factors for the analyte and the analyte-additive complex in the stationary phase. The terms including mobilities of analyte species in the stationary phase are eliminated from eq 1.5 .4 because the mobilities of species in the stationary phase are zero. The capacity factors for each species in Figure 1.5.1 are:

$k_{\mathrm{A}, \mathrm{M}}^{\prime}=1$

$k_{\mathrm{AC}, \mathrm{M}}^{\prime}=\mathrm{K}_{\mathrm{AC}, \mathrm{M}}[\mathrm{C}]$

$k_{\mathrm{A}, \mathrm{S}}^{\prime}=\mathrm{K}_{\mathrm{A}, \mathrm{S}}\left(\frac{\mathrm{V}_{\mathrm{S}}}{\mathrm{V}_{\mathrm{M}}}\right)$

$k_{\mathrm{AC}, \mathrm{S}}^{\prime}=\mathrm{K}_{\mathrm{AC}, \mathrm{M}}[\mathrm{C}] \mathrm{K}_{\mathrm{AC}, \mathrm{S}}\left(\frac{\mathrm{V}_{\mathrm{S}}}{\mathrm{V}_{\mathrm{M}}}\right)$

where $[\mathrm{C}]$ is the concentration of the additive in the buffer.

The capacity factors for each species indicate the tendency of the analyte to exist as a particular species in relation to the free analyte. Fig. 1.5.2(a) shows the capacity factors of the four analyte species, for a hypothetical equilibrium, over a range of additive concentrations. The capacity factors of the uncomplexed analyte species (in both the buffer and the stationary phase) are not affected by the additive concentration. Also, the ratio of $k_{\mathrm{A}, \mathrm{M}}^{\prime}$ and $k_{\mathrm{A}, \mathrm{S}}^{\prime}$ remains constant because the phase distribution of the uncomplexed analyte is independent of the additive concentration. 

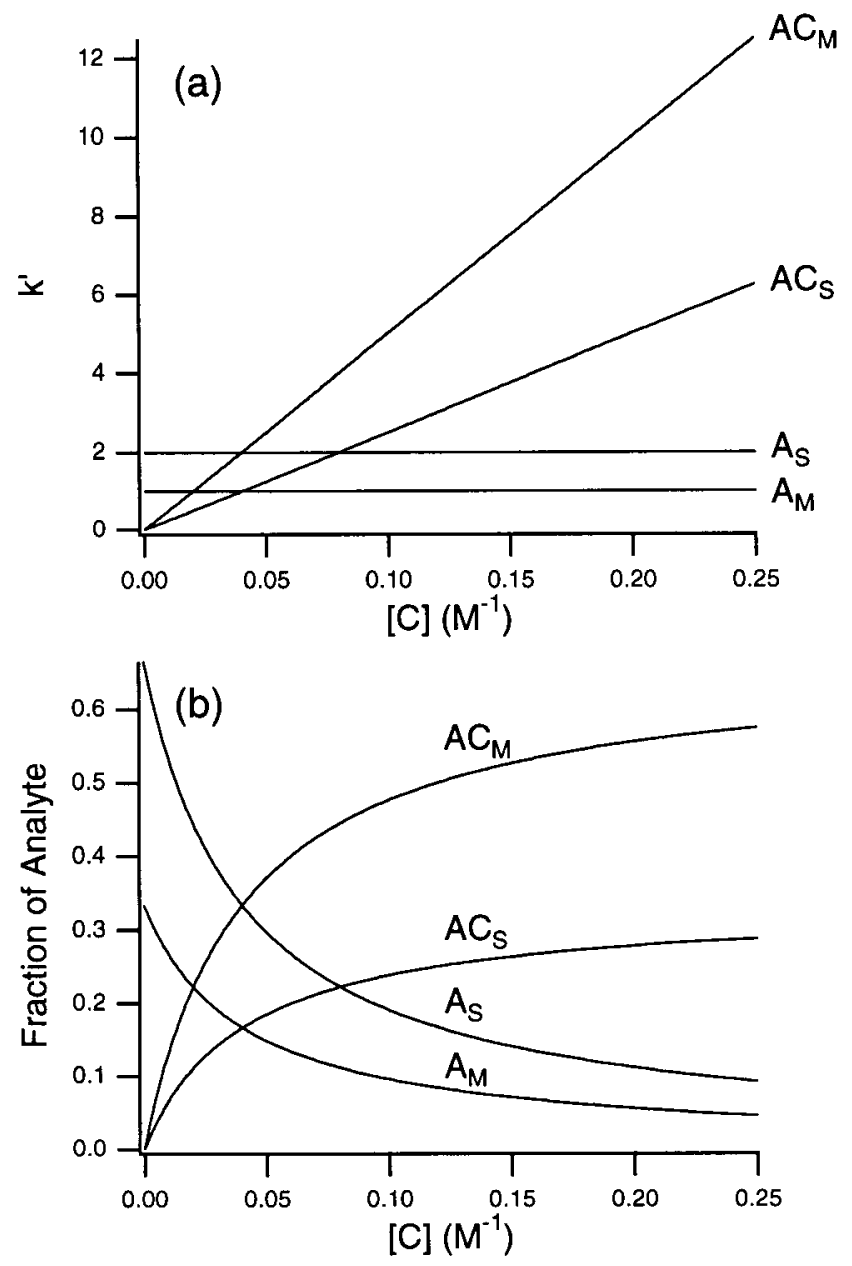

Figure 1.5.2 The effect of the additive concentration ([C]) on the capacity factors (a) and the fraction of analyte present as each species (b) for a system described by the equilibrium shown in Figure 1.5.1. The constants used to generate the curves are: $\mathrm{K}_{\mathrm{AC}, \mathrm{M}}=50 \mathrm{M}^{-1}, k_{\mathrm{A}, \mathrm{S}}^{\prime}=2$, and $\mathrm{K}_{\mathrm{AC}, \mathrm{S}}\left(\mathrm{V}_{\mathrm{S}} \mathrm{N}_{\mathrm{M}}\right)=0.5$.

The capacity factors of the complexed analyte species increase linearly with the additive concentration indicating that the tendency of the analyte to be complexed increases with additive concentration. The ratio of the $k_{\mathrm{AC}, \mathrm{M}}^{\prime}$ and $k_{\mathrm{AC}, \mathrm{S}}^{\prime}$ is constant because the phase distribution equilibrium of the analyte-additive complex is independent of the additive concentration.

Figure 1.5.2(b) shows the fraction of the analyte for each of the four species as a function of the additive concentration. The shapes of the curves in Figure 1.5.2(b) are quite different from the capacity factor curves in Figure 1.5.2(a). The capacity factors indicate the tendency the analyte to exist as a certain species in relation to the free analyte. As can be seen in Figure 
1.5.2(b) the fractions of the analyte species are not constant, nor do they change linearly with the additive concentration. If the curves in Figure 1.5.2(b) are divided by the curve for the free analyte $\left(A_{M}\right)$, the resulting curves would be identical to the capacity factor curves shown in Figure 1.5.2(a).

The points where the capacity factor curves in Figure 1.5.2(a) cross indicate that the tendency of the analyte to exist as those two species is equal at that additive concentration. Therefore, as shown in Fig. 1.5.2(b), the fraction of analyte present as any two species will be equal whenever the capacity factors for the two species are equal. Similarly, the ratio of the analyte present as any two species is equal to the ratio of their capacity factors. For example, the ratio of the analyte-additive complexes in the two phases is:

$$
\frac{n_{\mathrm{AC}, \mathrm{S}}}{n_{\mathrm{AC}, \mathrm{M}}}=\frac{k_{\mathrm{AC}, \mathrm{S}}^{\prime}}{k_{\mathrm{AC}, \mathrm{M}}^{\prime}}=\mathrm{K}_{\mathrm{AC}, \mathrm{S}}\left(\frac{\mathrm{V}_{\mathrm{S}}}{\mathrm{V}_{\mathrm{M}}}\right)
$$

The analyte migration behavior can be determined by inserting eqs $1.5 .5-1.5 .8$ into eq 1.5.4:

$$
\mu^{\mathrm{A}}=\frac{\mu_{\mathrm{A}, \mathrm{M}}+\mathrm{K}_{\mathrm{AC}, \mathrm{M}}[\mathrm{C}] \mu_{\mathrm{AC}, \mathrm{M}}}{1+\mathrm{K}_{\mathrm{AC}, \mathrm{M}}[\mathrm{C}]+\mathrm{K}_{\mathrm{A}, \mathrm{S}}\left(\frac{\mathrm{V}_{\mathrm{S}}}{\mathrm{V}_{\mathrm{M}}}\right)+\mathrm{K}_{\mathrm{AC}, \mathrm{M}}[\mathrm{C}] \mathrm{K}_{\mathrm{AC}, \mathrm{S}}\left(\frac{\mathrm{V}_{\mathrm{S}}}{\mathrm{V}_{\mathrm{M}}}\right)}
$$

The individual $k^{\prime}$ for a certain species is independent of capacity factors for other species of the analyte. For example, the $k^{\prime}$ for the uncomplexed analyte in the stationary phase is not affected by the presence of the complexation additive, allowing $k_{\mathrm{A}, \mathrm{S}}^{\prime}$ to be used for any additive at any concentration. This contrasts conventional chromatography theory where the capacity factor changes with the change in the additive concentration.

For a given column, the volumes of the stationary phase and the mobile phase remain constant, as well as the equilibrium constants for the phase distribution of the analyte and the analyte-additive complex. Therefore, eq 1.5 .10 can be simplified to: 


$$
\mu^{\mathrm{A}}=\frac{\mu_{\mathrm{A}, \mathrm{M}} *+\mathrm{K}_{\mathrm{AC}, \mathrm{M}} *[\mathrm{C}] \mu_{\mathrm{AC}, \mathrm{M}} *}{1+\mathrm{K}_{\mathrm{AC}, \mathrm{M}} *[\mathrm{C}]}
$$

where:

$$
\mu_{\mathrm{A}, \mathrm{M}} *=\frac{\mu_{\mathrm{A}, \mathrm{M}}}{1+\mathrm{K}_{\mathrm{A}, \mathrm{S}}\left(\frac{\mathrm{V}_{\mathrm{S}}}{\mathrm{V}_{\mathrm{M}}}\right)}
$$

$$
\mu_{\mathrm{AC}, \mathrm{M}} *=\frac{\mu_{\mathrm{AC}, \mathrm{M}}}{1+\mathrm{K}_{\mathrm{AC}, \mathrm{S}}\left(\frac{\mathrm{V}_{\mathrm{S}}}{\mathrm{V}_{\mathrm{M}}}\right)}
$$

$$
\mathrm{K}_{\mathrm{AC}, \mathrm{M}} *=\mathrm{K}_{\mathrm{AC}, \mathrm{M}}\left(\frac{1+\mathrm{K}_{\mathrm{AC}, \mathrm{S}}\left(\frac{\mathrm{V}_{\mathrm{S}}}{\mathrm{V}_{\mathrm{M}}}\right)}{1+\mathrm{K}_{\mathrm{A}, \mathrm{S}}\left(\frac{\mathrm{V}_{\mathrm{S}}}{\mathrm{V}_{\mathrm{M}}}\right)}\right)
$$

Eq. 1.5.11 is identical in form to the equation that describes a 1:1 interaction between an analyte and an additive in the absence of phase equilibria ${ }^{(35,62,66,73)}$. The binding isotherm for a 1:1 interaction in a CEC system should therefore take the same shape as a binding isotherm in the absence of the stationary phase. The apparent constants (eqs 1.5.11-1.5.13) for an analyteadditive interaction in a particular column can be solved for using a nonlinear regression or one of the linear transformations the same way as in $\mathrm{CE}^{(35,62,66,73)}$, allowing the effect of the additive on analyte mobility to be described. If the capacity factors for the analyte in the stationary phase and the analyte-additive complex in the stationary phase for a certain column are known, then the analyte migration behavior can be predicted using the true constants in eq 1.5.10. 


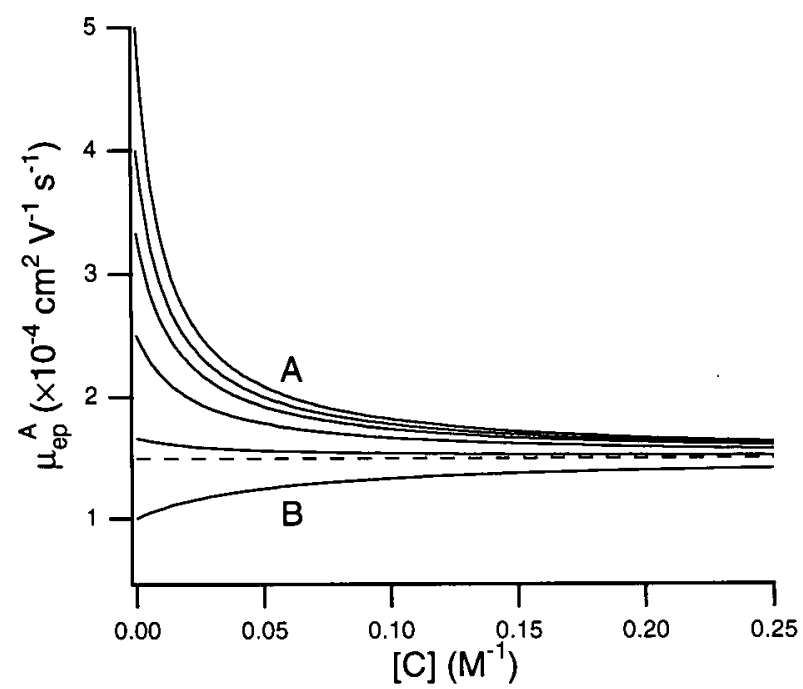

Figure 1.5.3 The effect of $k_{A, S}^{\prime}$ on the binding isotherms of an interaction between an analyte and an additive in a CEC system. The constants used to generate the curves are: $K_{A C, M}=50 M^{-1}, K_{A C, S}\left(V_{S} N_{M}\right)=1$, $\mu_{A, M}=5 \times 10^{-4} \mathrm{~cm}^{2} V^{-1} \mathrm{~s}^{-1}, \mu_{A C, M}=3 \times 10^{-4} \mathrm{~cm}^{2} V^{-1} \mathrm{~s}^{-1}$, and $k_{A, S}^{\prime}=0(A), 0.25,0.5$, 1,2 , and $4(B)$. The dashed line represents the binding isotherm where the analyte mobility is unaffected by the additive concentration $\left(k_{\mathrm{A} . \mathrm{S}}^{\prime}=\right.$ 2.35).

Although the migration behavior of an analyte in CEC is described by an equation that has the same form as in CE, even with the presence of a stationary phase, the apparent constants in this equation are affected by the phase distribution. Figure 1.5.3 shows the binding isotherms for a hypothetical interaction between an analyte and an additive with different capacity factors for the uncomplexed analyte in the stationary phase $\left(k_{\mathrm{A}, \mathrm{S}}^{\prime}\right)$. The interaction between the analyte and the additive does not change, but varying $k_{\mathrm{A}, \mathrm{S}}^{\prime}$ shifts the entire equilibrium. Fig. 1.5.4 shows the effect of $k_{\mathrm{A}, \mathrm{S}}^{\prime}$ on the apparent constants in eq 1.5.11. The apparent equilibrium constant $\left(\mathrm{K}_{\mathrm{AC}, \mathrm{M}}{ }^{*}\right)$ decreases as $k_{\mathrm{A}, \mathrm{S}}^{\prime}$ increases. As the affinity for the uncomplexed analyte towards the stationary phase increases, the entire equilibrium is shifted towards the uncomplexed analyte species, causing a decrease in $\mathrm{K}_{\mathrm{AC}, \mathrm{M}}{ }^{*}$. The apparent mobility of the free analyte is determined by the phase distribution of the uncomplexed analyte. As $k_{\mathrm{A}, \mathrm{S}}^{\prime}$ increases, the fraction of uncomplexed analyte in the buffer decreases, and the apparent mobility of the uncomplexed analyte also decreases. The apparent analyte-additive complex mobility is unaffected by $k_{\mathrm{A}, \mathrm{S}^{*}}$ 


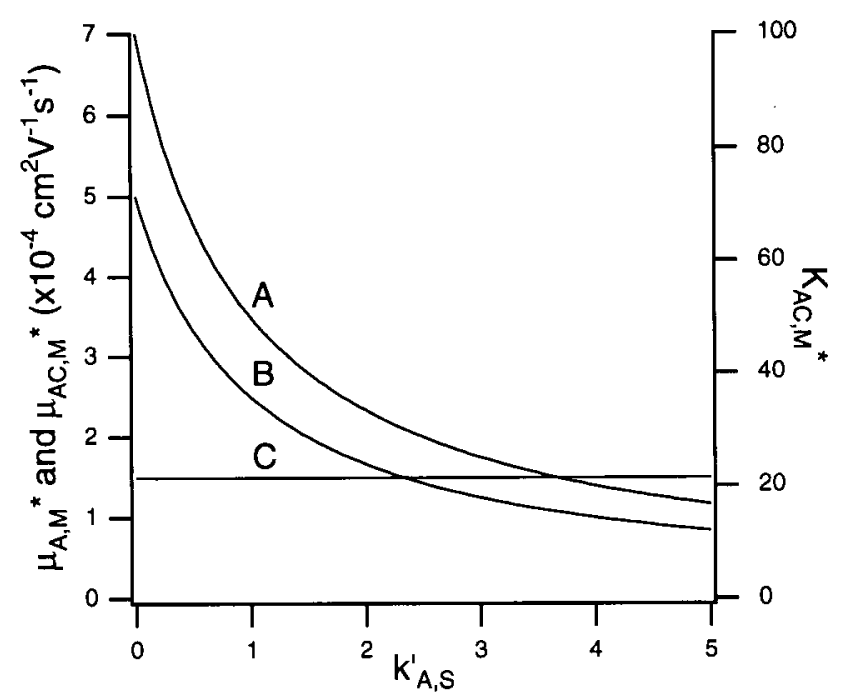

Figure 1.5.4 (a) The apparent binding constant $\left(\mathrm{K}_{A C, M}{ }^{*}\right)$, (b) the apparent analyte-additive complex mobility $\left(\mu_{\mathrm{AC}, M}{ }^{*}\right)$, and (c) the apparent uncomplexed analyte mobility $\left(\mu_{\mathrm{A}, \mathrm{M}}{ }^{*}\right)$ over a range of values for $k_{\mathrm{A}, \mathrm{S}^{\prime}}$. The constants used to generate the curves are: $\mathrm{K}_{\mathrm{AC}, \mathrm{M}}=50 \mathrm{M}^{-1}, \mathrm{~K}_{\mathrm{AC}, \mathrm{S}}\left(\mathrm{V}_{\mathrm{S}} \mathrm{V}_{\mathrm{M}}\right)=1$, $\mu_{\mathrm{A}, \mathrm{M}}=5 \times 10^{-4} \mathrm{~cm}^{2} \mathrm{~V}^{-1} \mathrm{~s}^{-1}$, and $\mu_{A C, M}=3 \times 10^{-4} \mathrm{~cm}^{2} \mathrm{~V}^{-1} \mathrm{~s}^{-1}$.

In Figure 1.5.4, the curves for the apparent free mobility and the apparent complex mobility intersect. At this $k_{\mathrm{A}, \mathrm{S}}^{\prime}$ value, $\mu_{\mathrm{A}}{ }^{*}$ is equal to $\mu_{\mathrm{AC}}{ }^{*}$, and the migration behavior of the analyte is not affected by the additive. The binding isotherm represented by the dashed line in Figure 1.5.3 corresponds to this case. At a certain value of the capacity factor for the free analyte in the stationary phase $\left(k_{\mathrm{A}, \mathrm{S}}^{\prime}\right)$, the additive does not affect the analyte mobility, even if there is a strong interaction between the analyte and the additive. This is analogous to the dengsu point discussed in chapter 1.4 where the addition of a second additive to a $\mathrm{CE}$ system does not affect the mobility of the analyte even though the second additive does alter the analyte mobility when used alone.

Figure 1.5.5 shows the effect of the phase distribution of the analyte-additive complex on the binding isotherms. The isotherms have shapes typical for a 1:1 interaction but the phase distribution of the complex clearly has a major effect on the apparent constants. 


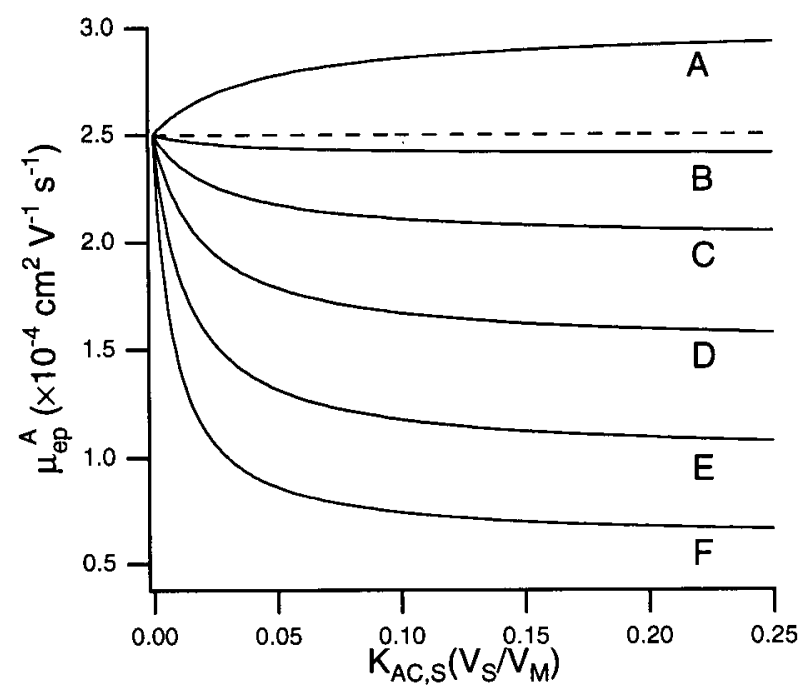

Figure 1.5.5 The effect of $K_{A C, S}\left(V_{S} N_{M}\right)$ on the binding isotherms of an interaction between an analyte and an additive in a CEC system. The constants used to generate the curves are: $\mathrm{K}_{\mathrm{AC}, \mathrm{M}}=50 \mathrm{M}^{-1}, k_{\mathrm{A}, \mathrm{S}}^{\prime}=1, \mu_{\mathrm{A}, \mathrm{M}}=$ $5 \times 10^{-4} \mathrm{~cm}^{2} V^{-1} \mathrm{~s}^{-1}, \mu_{\mathrm{AC}, \mathrm{M}}=3 \times 10^{-4} \mathrm{~cm}^{2} \mathrm{~V}^{-1} \mathrm{~s}^{-1}$, and $\mathrm{K}_{\mathrm{AC}, \mathrm{S}}\left(\mathrm{V}_{\mathrm{S}} \mathrm{N}_{\mathrm{M}}\right)=0(\mathrm{~A}), 0.25(\mathrm{~B})$, $0.5(C), 1(D), 2(E)$, and $4(F)$. The dashed line represents the binding isotherm where the analyte mobility is unaffected by the additive concentration $\left(\mathrm{K}_{\mathrm{ACC}, \mathrm{S}}\left(\mathrm{V}_{\mathrm{S}} \mathrm{N}_{\mathrm{M}}\right)=0.2\right)$.

Figure 1.5.6 shows how the apparent constants are affected by the complex phase distribution. As the amount of complex in the stationary phase increases, the entire equilibrium (Figure 1.5.1) shifts towards the complexed analyte species, causing an increase in the apparent equilibrium constant. The apparent complex mobility decreases as the affinity of the complex for the stationary phase increases. The apparent free analyte mobility is unaffected by the phase distribution of the analyte-additive complex. Again, there is a point in Figure 1.5.6 where the apparent free mobility and the apparent complex mobility are equal. At this point, the net analyte mobility is unaffected by the additive concentration, as demonstrated by the dashed line in Figure 1.5.5. 


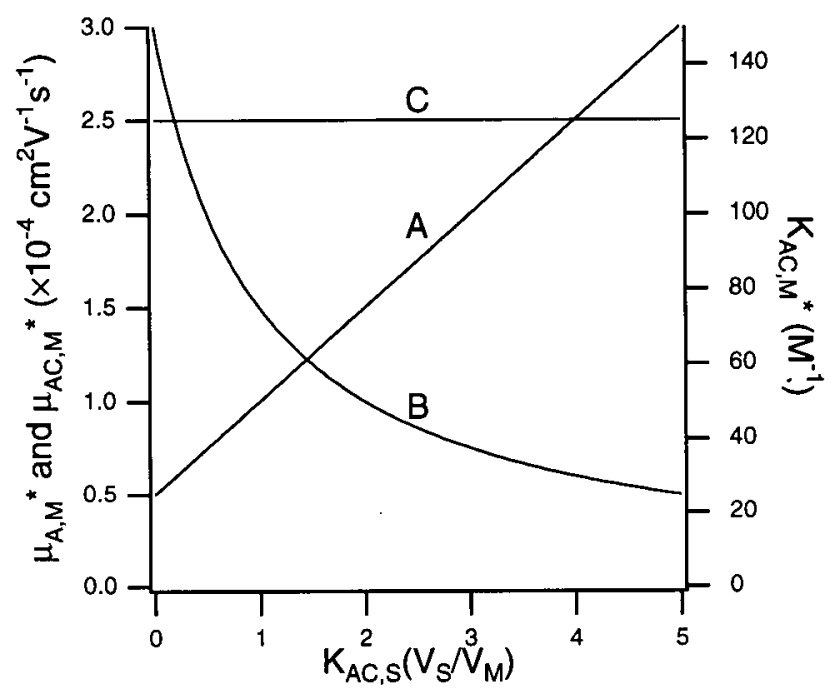

Figure 1.5.6 (A) The apparent binding constant $\left(\mathrm{K}_{\mathrm{ACM}}{ }^{*}\right)$, (B) the

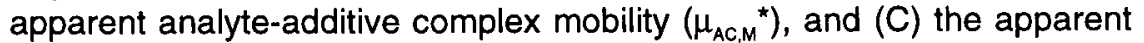
uncomplexed analyte mobility $\left(\mu_{A M}{ }^{*}\right.$ ) over a range of values for $\mathrm{K}_{\mathrm{AC}, \mathrm{S}}\left(\mathrm{V}_{\mathrm{S}} \mathrm{N}_{\mathrm{M}}\right)$. The constants used to generate the curves are: $\mathrm{K}_{\mathrm{AC}, \mathrm{M}}=50$ $\mathrm{M}^{-1}, k_{\mathrm{A}, \mathrm{S}}^{\prime}=1, \mu_{\mathrm{A}, \mathrm{M}}=5 \times 10^{-4} \mathrm{~cm}^{2} \mathrm{~V}^{-1} \mathrm{~s}^{-1}$, and $\mu_{\mathrm{AC}, \mathrm{M}}=3 \times 10^{-4} \mathrm{~cm}^{2} \mathrm{~V}^{-1} \mathrm{~s}^{-1}$.

\subsubsection{The Effect of the EOF on Analyte Mobility}

A distinguishing feature between $\mathrm{CE}$ and CEC is the effect of EOF on the analyte. The analyte migration rate in $\mathrm{CE}$ is determined by the sum of the net electrophoretic mobility $\left(\mu_{\mathrm{ep}}\right)$ and the electroosmotic mobility $\left(\mu_{\mathrm{e})}\right)$. Ideally, the electrophoretic mobilities of each analyte species remain constant when additives are used. Conversely, the electroosmotic mobility is often different for different capillaries, and difficult to predict in the presence of additives. Because the effect of additives on the electroosmotic mobility is difficult to predict, it is often more reasonable to describe the migration behavior of analytes in terms of electrophoretic mobilities since they are intrinsic to the analyte species.

In CE, all of the analyte species are equally affected by the EOF. The net electrophoretic mobility of an analyte can be obtained by subtracting the $\mu_{\mathrm{e} 0}$ directly from the net mobility of the analyte in eq 1.5.2. The analyte migration behavior can then be described in terms of electrophoretic mobility: 


$$
\mu^{\mathrm{A}}-\mu_{\mathrm{eo}}=\mu_{\mathrm{ep}}^{\mathrm{A}}=\frac{\sum_{\mathrm{i}=1}^{\mathrm{m}} k_{\mathrm{i}}^{\prime} \mu_{\mathrm{ep}, \mathrm{i}}}{\sum_{\mathrm{i}=1}^{\mathrm{m}} k_{\mathrm{i}}^{\prime}}
$$

In CEC, however, not all of the analyte species experience the EOF. Therefore, in CEC it is not possible to describe the analyte migration behavior in terms of electrophoretic mobility simply by subtracting $\mu_{\mathrm{co}}$ from the net analyte mobility. Since the analyte is affected by EOF only when it is in the mobile phase, the net electrophoretic mobility of the analyte $\left(\mu_{\mathrm{ep}}^{\mathrm{A}}\right)$ in CEC is determined by:

$$
\mu^{\mathrm{A}}-f_{\mathrm{M}} \mu_{\mathrm{eo}}=\mu_{\mathrm{ep}}^{\mathrm{A}}=\frac{\sum_{\mathrm{i}=1}^{\mathrm{n}} k_{\mathrm{i}}^{\prime} \mu_{\mathrm{ep}, \mathrm{i}}}{\sum_{\mathrm{i}=1}^{\mathrm{n}} k_{\mathrm{i}}^{\prime}}
$$

where $f_{\mathrm{M}}$ is the fraction of analyte in the mobile phase, which is determined by the capacity factors of the analyte species:

$$
f_{\mathrm{M}}=\frac{\sum k^{\prime} \text { of analyte species in the mobile phase }}{\sum k^{\prime} \text { of all analyte species }}
$$

Describing analyte mobility, when the EOF is not a constant, is more difficult in CEC than in $\mathrm{CE}$ because the capacity factors should be calculated from the net electrophoretic mobilities of the analyte at different additive concentrations. However, without the values of the capacity factors, it is impossible to accurately correct for the EOF.

In the simplest case where there is no additive in the buffer, eqs 1.5 .16 and 1.5 .17 can be used to express the net electrophoretic mobility of the analyte as:

$$
\mu_{\mathrm{ep}}^{\mathrm{A}}=\mu^{\mathrm{A}}-\frac{1}{1+k_{\mathrm{A}, \mathrm{S}}^{\prime}} \mu_{\mathrm{eo}}=\frac{1}{1+k_{\mathrm{A}, \mathrm{S}}^{\prime}} \mu_{\mathrm{A}, \mathrm{M}}
$$

Note that $\mu_{A, M}$ in this section is the electrophoretic mobility of the free analyte in the mobile 
phase. For neutral analytes, eq 1.5 .18 simplifies to:

$\mu^{\mathrm{A}}=\frac{1}{1+k_{\mathrm{A}, \mathrm{S}}^{\prime}} \mu_{\mathrm{eo}}$

The value of $\mu_{\mathrm{ep}}^{\mathrm{A}}$, in this case, is zero. The capacity factor for the analyte in the stationary phase can be found by measuring the net mobility of the analyte and the EOF for a single CEC run.

When the analyte is charged, eq 1.5 .18 cannot be simplified to eq 1.5 .19 and it is impossible to determine $k_{\mathrm{A} . \mathrm{S}}^{\prime}$ using CEC alone. The capacity factor for the analyte in the stationary phase can be determined by capillary liquid chromatography using pressure (instead of electric field) to drive the analyte through the column. If the buffer is the same then the net migration rate of the analyte is:

$\overline{\mathrm{U}}_{\mathrm{A}}=\frac{1}{1+k_{\mathrm{A}, \mathrm{S}}^{\prime}} \overline{\mathrm{U}}_{\mathrm{o}}$

$k_{\mathrm{A}, \mathrm{S}}^{\prime}$ can be calculated from eq 1.5 .20 by comparing the net migration rate of the analyte and the migration rate of a marker that does not interact with the stationary phase. Once $k_{\mathrm{A} . \mathrm{S}}^{\prime}$ is known, $\mu_{\mathrm{A}, \mathrm{M}}$ can be calculated using eq 1.5 .18 after $\mu^{\mathrm{A}}$ and $\mu_{\mathrm{e} o}$ are measured.

If there is an additive in the buffer (as in Figure 1), eqs 1.5.16 and 1.5.17 can be used to determine the net electrophoretic mobility of the analyte in a particular column:

$\mu_{\mathrm{ep}}^{\mathrm{A}}=\mu^{\mathrm{A}}-\frac{1+k_{\mathrm{AC}, \mathrm{M}}^{\prime}}{1+k_{\mathrm{AC}, \mathrm{M}}^{\prime}+k_{\mathrm{A}, \mathrm{S}}^{\prime}+k_{\mathrm{AC}, \mathrm{S}}^{\prime}} \mu_{\mathrm{eo}}=\frac{\mu_{\mathrm{ep}, \mathrm{A}, \mathrm{M}} * \mathrm{~K}_{\mathrm{AC}, \mathrm{M}} *[\mathrm{C}] \mu_{\mathrm{ep}, \mathrm{AC}, \mathrm{M}} *}{1+\mathrm{K}_{\mathrm{AC}, \mathrm{M}} *[\mathrm{C}]}$

Based on eq 1.5.14, eq 1.5.21 can be rearranged to:

$\mu_{\mathrm{ep}}^{\mathrm{A}}=\mu^{\mathrm{A}}-\frac{1+\mathrm{K}_{\mathrm{AC}, \mathrm{M}}[\mathrm{C}]}{\left(1+\mathrm{K}_{\mathrm{AC}, \mathrm{M}} *[\mathrm{C}]\right)\left(1+k_{\mathrm{A}, \mathrm{S}}^{\prime}\right)} \mu_{\mathrm{eo}}=\frac{\mu_{\mathrm{ep}, \mathrm{A}, \mathrm{M}} *+\mathrm{K}_{\mathrm{AC}, \mathrm{M}} *[\mathrm{C}] \mu_{\mathrm{ep}, \mathrm{AC}, \mathrm{M}} *}{1+\mathrm{K}_{\mathrm{AC}, \mathrm{M}} *[\mathrm{C}]}$

The effect of the additive on the net electrophoretic mobility of the analyte is described by the equation for a 1:1 binding isotherm given in eq 1.5.22. If the EOF is not constant when 
the additive concentration is changed, then the total net mobility must be corrected to give the electrophoretic mobility. Although the effect of the additive on the electrophoretic mobility can be described using an apparent binding isotherm, the phase distribution of the analyte species must be considered in order to calculate $\mu_{\mathrm{ep}}^{\mathrm{A}}$ from $\mu^{\mathrm{A}}$. The value of $k_{\mathrm{A}, \mathrm{S}}^{\prime}$ can be obtained using pressure to drive the analyte in the column as in eq 1.5.20.

The value of $\mathrm{K}_{\mathrm{AC}, \mathrm{M}}$ can be determined by $\mathrm{CE}$ in the absence of the stationary phase since the true equilibrium constant is independent of the phase distribution. The unified separation theory allows the individual capacity factors to be exchanged from one technique to another because they describe the same physicochemical parameters. The net electrophoretic mobility in the absence of the stationary phase is:

$\mu_{\mathrm{ep}}^{\mathrm{A}}=\mu^{\mathrm{A}}-\mu_{\mathrm{eo}}=\frac{\mu_{\mathrm{ep}, \mathrm{A}}+\mathrm{K}_{\mathrm{AC}, \mathrm{M}}[\mathrm{C}] \mu_{\mathrm{ep}, \mathrm{AC}}}{1+\mathrm{K}_{\mathrm{AC}, \mathrm{M}}[\mathrm{C}]}$

where $\mathrm{K}_{\mathrm{AC}, \mathrm{M}}$ can be calculated using a nonlinear regression or one of the linear transformations of this equation ${ }^{(35,62,66,73)}$, using the $\mu^{\mathrm{A}}$ and $\mu_{\mathrm{e} 0}$ values measured at various additive concentrations. The mobilities of the free and complexed analyte determined in open tubular CE (eq 1.5.23) cannot be used in CEC because although the mobilities should be same, the analytes do not travel in a straight path due to the presence of the packing material, resulting in different apparent mobilities.

$\mathrm{K}_{\mathrm{AC}, \mathrm{M}}{ }^{*}$ is the only remaining unsolved constant necessary to correct for the EOF in eq 1.5.22. Unfortunately, if the EOF is not constant, $\mathrm{K}_{\mathrm{AC}, \mathrm{M}}$ * can not be solved without correcting for the EOF. In this case, an iterative process is necessary to solve for the apparent constants in eq 1.5.22. Initially the net electrophoretic mobility $\left(\mu_{\mathrm{ep}}^{\mathrm{A}}\right)$ should be estimated using the measured values of $\mu^{\mathrm{A}}$ and $\mu_{\mathrm{eo}}$, and the values of $k_{\mathrm{A}, \mathrm{S}}^{\prime}$ and $\mathrm{K}_{\mathrm{AC}, \mathrm{M}}$ calculated from other methods, and an appropriate estimate for $\mathrm{K}_{\mathrm{AC}, \mathrm{M}}{ }^{*}\left(\right.$ e.g. $50 \mathrm{M}^{-1}$ ). After the $\mu_{\mathrm{ep}}^{\mathrm{A}}$ are measured at several additive 
concentrations, the apparent constants in eq 1.5 .22 can be estimated using the nonlinear regression method or one of the linear transformations of the mobility equation ${ }^{(35,62,66,73)}$. This will give a better estimate for $\mathrm{K}_{\mathrm{AC}, \mathrm{M}}{ }^{*}$, allowing an improved correction for the EOF and a better estimate of the $\mu_{\mathrm{ep}}^{\mathrm{A}}$ measured at the different additive concentrations. These values of $\mu_{\mathrm{ep}}^{\mathrm{A}}$ can then be used to calculate an even better estimate of $\mathrm{K}_{\mathrm{AC,M}}{ }^{*}$. This process should be continued until the estimate for $\mathrm{K}_{\mathrm{AC}, \mathrm{M}}{ }^{*}$ converges. The true electrophoretic mobilities and capacity factors for each analyte species can then be calculated from the apparent constants and the values of $k_{\mathrm{A}, \mathrm{S}}^{\prime}$ and $\mathrm{K}_{\mathrm{AC}, \mathrm{M}}$ measured independently.

\subsubsection{The Effect of the EOF on Resolution}

The EOF has a large effect on the resolution of a pair of analytes. In CE, where all of the analyte species are affected by the EOF, $\gamma$ can be expressed as:

$$
\gamma=\frac{\mu^{\mathrm{A}}}{\mu^{\mathrm{B}}}=\frac{\mu_{\mathrm{ep}}^{\mathrm{A}}+\mu_{\mathrm{eo}}}{\mu_{\mathrm{ep}}^{\mathrm{B}}+\mu_{\mathrm{eo}}}
$$

When the EOF increases the magnitude of $\mu^{\mathrm{A}}$ and $\mu^{\mathrm{B}}, \gamma$ decreases; if the EOF acts as a counter flow and decreases the magnitude of $\mu^{\mathrm{A}}$ and $\mu^{\mathrm{B}}, \gamma$ increases with the increase in EOF. The change in EOF affects the resolution by changing the ratio of mobilities which, as demonstrated in section 1.2 .5 , is linearly related to the resolution. The EOF does not change the difference between $\mu^{\mathrm{A}}$ and $\mu^{\mathrm{B}}$, demonstrating that $\Delta \mu$ is an inadequate estimate of resolution.

In CEC, not all of the analyte species are affected by the EOF. The distribution of the analyte between species in the buffer and species in the stationary phase determines the effect of EOF on the migration behavior of each analyte. The separation factor $(\gamma)$ in CEC can therefore be expressed as: 
88.

$\gamma=\frac{\mu_{\mathrm{ep}}^{\mathrm{A}}+f_{\mathrm{M}}^{\mathrm{A}} \mu_{\mathrm{eo}}}{\mu_{\mathrm{ep}}^{\mathrm{B}}+f_{\mathrm{M}}^{\mathrm{B}} \mu_{\mathrm{eo}}}$

where $f_{\mathrm{M}}^{\mathrm{A}}$ and $f_{\mathrm{M}}^{\mathrm{B}}$ are the fractions of analytes $\mathrm{A}$ and $\mathrm{B}$ in the buffer. Because the fraction of each analyte in the buffer can be different, the EOF can change both the difference and the ratio of the two mobilities.

The most obvious example is when both analytes are neutral. In this case, $\gamma$ is given by:

$\gamma=\frac{f_{\mathrm{M}}^{\mathrm{A}}}{f_{\mathrm{M}}^{\mathrm{B}}}$

There is no field contribution to the separation because both analytes are neutral. The EOF in this case truly acts as a "pump" and the resolution is solely determined by the phase distribution of the analytes.

In another case, where there is no EOF, the migration of the analytes is determined by their electrophoretic mobilities, and $\gamma$ is equal to:

$\gamma=\frac{\mu_{\mathrm{ep}}^{\mathrm{A}}}{\mu_{\mathrm{ep}}^{\mathrm{B}}}$

where $\mu_{\mathrm{ep}}^{\mathrm{A}}$ and $\mu_{\mathrm{ep}}^{\mathrm{B}}$ are effected by both the distribution of the analytes between species and the individual mobilities of each species. It is clear that in CEC, separation can be achieved in two ways: 1) electrophoretic separation, which is determined by both equilibrium and field effects; and 2) electroosmotic separation, which is determined solely by the distribution (equilibrium) between a mobile phase and a stationary phase.

\subsubsection{Correction Factor}

Equation 1.5.1 describes the analyte migration behavior in the ideal situation where any 
change in the net analyte migration rate is caused by shifts in the equilibria involved in the separation system. In reality, using additives in a separation system such as CEC often changes the viscosity and other properties of the background electrolyte. Both the electrophoretic mobilities of the analyte species and electroosmotic mobility are affected by these changes. This has led to the use of a correction factor $(v)$ in $C E$ to normalize the net analyte mobility to the ideal state where the additive concentration approaches zero ${ }^{(35)}$. A similar strategy can be used in CEC allowing eq 1.5.2 to be written as:

$$
v \mu_{\mathrm{ep}}^{\mathrm{A}}=\frac{\sum_{\mathrm{i}=1}^{\mathrm{m}} k_{\mathrm{i}}^{\prime} \mu_{\mathrm{i}}}{\sum_{\mathrm{i}=1}^{\mathrm{m}} k_{\mathrm{i}}^{\prime}}
$$

Unlike the compensation for the EOF, only the net analyte electrophoretic mobility needs to be corrected in this equation. Although the changes in properties such as viscosity only affect the mobilities of the analyte species in the buffer, all of the analyte species not in the buffer have a mobility of zero. The result is that the mobilities of the analyte species in the buffer are corrected for the viscosity change while the mobilities of the species in the stationary phase remain zero. This contrasts the EOF correction because EOF is added to the electrophoretic mobilities while the correction factor is multiplicative.

\subsubsection{Conclusions}

This chapter has demonstrated the usefulness of the generalized separation theory presented in chapter 1.2. Hybrid systems such as CEC are not easily described using conventional theories. The ability of the unified separation theory to describe systems with complicated equilibria and/or analyte species with different migration velocities simplifies the description of hybrid techniques. Also, using the individual capacity factors to describe each 
90.

interaction involved in a separation system makes it possible to apply physicochemical constants measured using different methods interchangeably. The theory presented in this chapter should facilitate the design of complex separation systems such as CEC, allowing better separation of closely related chemicals. 


\subsection{References}

(1) Tswett, M. S. Michael Tswett's First Paper on Chromatography; M. Woelm, Eschwege: Germany, 1954.

(2) Martin, A. J. P.; Synge, R. L. M. Biochem. J. 1941, 35, 1358-68.

(3) James, A. T.; Martin, A. J. P. Biochem. J. 1952, 50, 679-90.

(4) Hjertén, S. Electrophoresis 1988, 9, 3-15.

(5) Svedberg, T.; Rinde, H. J. Am. Chem. Soc. 1924, 46, 2677-93.

(6) Tiselius, A. The moving boundary method of studying the electrophoresis of proteins (Thesis); Almqvist \& Wiksell: Uppsala, 1930.

(7) Hjertén, S. Chromatogr. Rev. 1967, 9, 122-219.

(8) Jorgenson, J. W.; Lukacs, K. D. Anal. Chem. 1981, 53, 1298-302.

(9) Jorgenson, J. W.; Lukas, K. D. J. Chromatogr. 1981, 218, 209-16.

(10) Giddings, J. C. Sep. Sci. 1966, 1, 123-5.

(11) Giddings, J. C. Sep. Sci. Tech. 1985, 19, 831-47.

(12) Caldwell, K. D. Anal. Chem. 1988, 60, 959A-71A.

(13) Terabe, S.; Otsuka, K.; Ichikawa, K.; Tsuchiya, A.; Ando, T. Anal. Chem. 1984, 56, 111-3.

(14) Terabe, S.; Otsuka, K.; Ando, T. Anal. Chem. 1985, 57, 834-41.

(15) Pretorius, V.; Hopkins, B. J.; Schieke, J. D. J. Chromatogr. 1974, 99, 23-30.

(16) Knox, J. H. J. Chromatogr. A 1994, 680, 3-13.

(17) Strain, H. H.; Murphy, G. W. Anal. Chem. 1952, 24, 50-60.

(18) Strain, H. H.; Sato, T. R.; Engelke, J. Anal. Chem. 1954, 26, 90-100.

(19) Giddings, J. C. Sep. Sci. Tech. 1978, 13, 3-24.

(20) Giddings, J. C. Sep. Sci. Tech. 1979, 14, 871-82.

(21) Giddings, J. C. Anal. Chem. 1981, 53, 945A-52A.

(22) Giddings, J. C. Unified Separation Science; Wiley-Interscience Publication: New York, 1991.

(23) Longsworth, L. G. In Electrophoresis Theory, Methods, and Applications; Bier, M., Ed.; Academic Press Inc.: New York, 1959, pp 95-106.

(24) Wilson, N. J. Am. Chem. Soc. 1940, 62, 1583-91.

(25) Ståhlberg, J. Anal. Chem. 1997, 69, 3812-21.

(26) Hjertén, S. Electrophoresis 1990, 11, 665-90.

(27) Hjertén, S. Nucleosides Nulceotides 1990, 9, 319-30.

(28) Hjertén, S.; Ericson, C.; Li, Y.-M.; Zhang, R. Biomed. Chromatogr. 1998, 12, in press.

(29) Rathore, A. S.; Horvath, C. J. Chromatogr. A 1996, 743, 231-46.

(30) Culbertson, C. T.; Jorgenson, J. W. Anal. Chem. 1994, 66, 955-62.

(31) Bowser, M. T.; Sternberg, E. D.; Chen, D. D. Y. Anal. Biochem. 1996, 241, 143-50.

(32) Bowser, M. T.; Bebault, G. M.; Peng, X.; Chen, D. D. Y. Electrophoresis 1997, 18, 2928-34. 
(33) Florence, T. M. Talanta 1982, 29, 345-64.

(34) Karger, B. L.; Snyder, L. R.; Horvath, C. An Introduction to Separation Science; Interscience: N.Y., 1973.

(35) Peng, X.; Bowser, M. T.; Britz-McKibbin, P.; Bebault, G. M.; Morris, J.; Chen, D. D. Y. Electrophoresis 1997, 18, 706-16.

(36) Peng, X.; Bebault, G. M.; Sacks, S. L.; Chen, D. D. Y. Can. J. Chem. 1997, 75, 507-17.

(37) Armstrong, D. W.; Nome, F. Anal. Chem. 1981, 53, 1662-6.

(38) Ruthven, D. M. Principles of Adsorption and Adsorption Processes; John Wiley \& Sons: New York, 1984.

(39) Suzuki, M. Adsorption Engineering; Elsevier: New York, 1990.

(40) Tien, C. Adsorption Calculations and Modeling; Butterworth-Heinemann: Boston, 1994.

(41) Mazzeo, J. R.; Swartz, M. E.; Grover, E. R. Anal. Chem. 1995, 67, 2966-73.

(42) Kenndler, E. J. Cap. Elec. 1996, 3, 191-8.

(43) Terabe, S.; Ozaki, H.; Otsuka, K.; Ando, T. J. Chromatogr. 1985, 332, 211-7.

(44) Guttman, A.; Paulus, A.; Cohen, A. S.; Grinberg, N.; Karger, B. L. J. Chromatogr. 1988, 448, 4153.

(45) Wren, S. A. C.; Rowe, R. C. J. Chromatogr. 1992, 603, 235-41.

(46) Esaka, Y.; Yamaguchi, Y.; Kano, K.; Goto, M.; Haraguchi, H.; Takahashi, J. Anal. Chem. 1994, 66, 2441-5.

(47) Okada, T. J. Chromatogr. A 1995, 695, 309-17.

(48) Rundlett, K. L.; Armstrong, D. W. Anal. Chem. 1995, 67, 2088-95.

(49) Armstrong, D. W.; Rundlett, K. L.; Reid, G. L. Anal. Chem. 1994, 66, 1690-5.

(50) Armstrong, D. W.; Gasper, M. P.; Rundlett, K. L. J. Chromatogr. A 1995, 689, 285-304.

(51) Liu, J.; Volk, K. J.; Lee, M. S.; Kerns, E. H.; Rosenberg, I. E. J. Chromatogr. A 1994, 680, 395403.

(52) Honda, S.; Taga, A.; Suzuki, K.; Suzuki, S.; Kakehi, K. J. Chromatogr. 1992, 597, 377-82.

(53) Kuhn, R.; Frei, R.; Christen, M. Anal. Biochem. 1994, 218, 131-5.

(54) Wren, S. A. C.; Rowe, R. C. J. Chromatogr. 1992, 609, 363-7.

(55) Wren, S. A. C. J. Chromatogr. 1993, 636, 57-62.

(56) Penn, S. G.; Goodall, D. M.; Loran, J. S. J. Chromatogr. 1993, 636, $149-52$.

(57) Rawjee, Y. Y.; Williams, R. L.; Vigh, G. J. Chromatogr. 1993, 635, 291-306.

(58) Shibukawa, A.; Lloyd, D. K.; Wainer, I. W. Chromatographia 1993, 35, 419-29.

(59) Penn, S. G.; Bergstrom, E. T.; Goodall, D. M.; Loran, J. S. Anal. Chem. 1994, 66, 2866-73.

(60) Lurie, I. S.; Klein, R. F. X.; Dalcason, T. A.; Lebelle, M. J.; Brenneisen, R.; Weinberger, R. E. Anal. Chem. 1994, 66, 4019-26.

(61) Penn, S. G.; Bergstrom, E. T.; Knights, I.; Liu, G.; Ruddick, A.; Goodall, D. M. J. Phys. Chem. 1995, 99, 3875-80.

(62) Rundlett, K. L.; Armstrong, D. W. J. Chromatogr. A 1996, 721, 173-86.

(63) Britz-Mckibbon, P.; Chen, D. D. Y. J. Chromatogr. A 1997, 781, 23-34. 
(64) Liu, J.; Volk, K. J.; Lee, M. S.; Pucci, M.; Handwerger, S. Anal. Chem. 1994, 66, 2412-6.

(65) Heegaard, N. H. J. Chromatogr. A 1994, 680, 405-12.

(66) Bowser, M. T.; Sternberg, E. D.; Chen, D. D. Y. Electrophoresis 1997, 18, 82-91.

(67) Wang, F.; Khaledi, M. G. Anal. Chem. 1996, 68, 3460-7.

(68) Vogt, C.; Conradi, S. Anal. Chim. Acta 1994, 294, 145-53.

(69) Mammen, M.; Gomez, F. A.; Whitesides, G. M. Anal. Chem. 1995, 67, 3526-35.

(70) Conradi, S.; Vogt, C.; Wittrisch, H.; Knobloch, G.; Werner, G. J. Chromatogr. A 1996, 745, 1039.

(71) Sänger - van de Griend, C. Ninth International Symposium on High Performance Capillary Electrophoresis (HPCE '97), 1997; poster 452.

(72) Deranleau, D. A. J. Am. Chem. Soc. 1969, 91, 4050-4.

(73) Connors, K. A. Binding Constants - The Measurement of Molecular Complex Stability; John Wiley \& Sons: Toronto, 1987.

(74) Kortüm, G.; Vogel, W.; Andrusson, K. Dissociation Constants of Organic Acids in Aqueous Solution; Butterworths: London, 1961.

(75) Cadogan, J. I. G.; Ley, S. V.; Pattenden, G.; Raphael, R. A.; Rees, C. W. Dictionary of Organic Compounds; New York, 1996.

(76) Deranleau, D. A. J. Am. Chem. Soc. 1969, 91, 4044-9.

(77) Rasmussen, H. T.; Goebel, L. K.; McNair, H. M. J. Chromatogr. 1990, 517, 549-55.

(78) Little, E. L.; Foley, J. P. J. Microcol. Sep. 1992, 4, 145-54.

(79) Kranack, A. R.; Bowser, M. T.; Britz-McKibbon, P.; Chen, D. D. Y. Electrophoresis 1998, 19, 388-96.

(80) Vandenbosch, S. E.; Heemstra, S.; Kraak, J. C.; Poppe, H. J. Chromatogr. A 1996, 755, 165-77.

(81) Lelievre, F.; Yan, C.; Zare, R. N.; Gareil, P. J. Chromatogr. A 1996, 723, 145-56.

(82) Yamamoto, H.; Baumann, J.; Erni, F. J. Chromatogr. A 1992, 593, 313-9.

(83) Knox, J. H.; Grant, I. H. Chromatographia 1987, 24, 135-43.

(84) Knox, J. H.; Grant, I. H. Chromatographia 1991, 32, 317-28.

(85) Li, S.; Lloyd, D. K. J. Chromatogr. A 1994, 666, 321-35. 


\section{Part 2: Monte Carlo Simulation of Error Propagation in the Determination of Binding Constants from Rectangular Hyperbolae}

\subsection{Background}

Over the past 85 years rectangular hyperbolae have been used to describe a variety of physicochemical properties influenced by chemical equilibria ${ }^{(1)}$ including spectrophotometry(2-7), NMR chemical shifts( ${ }^{(8-13)}$, Michaelis-Menten kinetics ${ }^{(14-21)}$, ion transport across membranes ${ }^{(22)}$, pharmacokinetics ${ }^{(23-26)}$ and even algal growth $\operatorname{rates}^{(27-29)}$. All of these studies are based on a certain chemical response dictated by a 1:1 interaction between a substrate and a ligand. A rectangular hyperbola generally can be expressed as ${ }^{(1)}$ :

$$
y=\frac{d x}{f+e x}
$$

where $y$ is the response, $x$ is the ligand concentration, and $\mathrm{d}, \mathrm{e}$, and, $\mathrm{f}$ are constants. One of the constants is redundant since eq 2.1 .1 can be rearranged, allowing the response to be described using two constants. One constant describes the equilibrium (the binding constant or dissociation constant) while the other describes the maximum response range (i.e. the difference between the response at zero and infinite ligand concentrations). Equation 2.1.1 can be expressed using either association constants (common in spectroscopic studies) or dissociation constants (common in kinetic and biochemical studies).

Equations taking the general form of eq 2.1.1 have been developed independently in many research areas including spectrophotometry (eq 2.1.2), NMR (eq 2.1.3), and MichaelisMenten kinetics (eq 2.1.4). When spectrophotometric methods are used, eq. 2.1.1 is usually expressed as:

$$
\frac{\left(\mathrm{A}-\mathrm{A}_{\mathrm{S}}\right)}{\mathrm{b}}=\frac{\left[\mathrm{S}_{0}\right]\left(\varepsilon_{\mathrm{SL}}-\varepsilon_{\mathrm{S}}\right) \mathrm{K}[\mathrm{L}]}{1+\mathrm{K}[\mathrm{L}]}
$$

where $\mathrm{A}$ is the measured absorbance, $\mathrm{A}_{\mathrm{s}}$ is the absorbance of the substrate in the absence of 
ligand, $\mathrm{b}$ is the optical path length, $\left[\mathrm{S}_{0}\right]$ is the initial concentration of the substrate, $\varepsilon_{\mathrm{SL}}$ and $\varepsilon_{\mathrm{S}}$ are the molar absorbtivities of the substrate-ligand complex and the substrate respectively, $\mathrm{K}$ is the binding constant and $[\mathrm{L}]$ is the ligand concentration.

If NMR is used to study chemical binding, eq 2.1.1 is usually written as:

$$
\left(\delta-\delta_{S}\right)=\frac{\left(\delta_{S L}-\delta_{S}\right) K[L]}{1+K[L]}
$$

where $\delta$ is the measured chemical shift, $\delta_{\mathrm{S}}$ and $\delta_{\mathrm{SL}}$ are the chemical shifts of the substrate and the substrate-ligand complex respectively, $\mathrm{K}$ is the binding constant, and $[\mathrm{L}]$ is the ligand concentration.

Michaelis-Menten kinetics presents eq 2.1.1 as:

$$
\mathrm{v}=\frac{\mathrm{V}_{\mathrm{m}}[\mathrm{S}]}{\mathrm{K}_{\mathrm{m}}+[\mathrm{S}]}
$$

where $\mathrm{v}$ is the observed rate of the reaction, $\mathrm{V}_{\mathrm{m}}$ is the maximum rate of the reaction achieved when the catalyst is fully complexed, [S] is the concentration of the substrate, and $\mathrm{K}_{\mathrm{m}}$ is the dissociation constant. Equation 2.1.4 takes a slightly different form than eqs 2.1.2 and 2.1.3 because it is expressed according to the dissociation constant $\left(\mathrm{K}_{\mathrm{m}}=1 / \mathrm{K}\right)$.

Recently, it has been shown that in capillary electrophoresis (CE), analyte mobility in the presence of analyte-additive interactions can be described according to ${ }^{(30-40)}$ :

$$
\left(v \mu_{e p}^{\mathrm{A}}-\mu_{\mathrm{ep}, \mathrm{A}}\right)=\frac{\left(\mu_{\mathrm{ep}, \mathrm{AC}}-\mu_{\mathrm{ep}, \mathrm{A}}\right) \mathrm{K}_{\mathrm{AC}}[\mathrm{C}]}{1+\mathrm{K}_{\mathrm{AC}}[\mathrm{C}]}
$$

where $\mu_{\mathrm{ep}}^{\mathrm{A}}$ is the net electrophoretic mobility of the analyte, $\nu$ is a correction factor which normalizes $\mu_{\mathrm{ep}}^{\mathrm{A}}$ to conditions where [C] approaches zero, [C] is the concentration of the complexation additive (analogous to $[\mathrm{L}]$ ), $\mathrm{K}_{\mathrm{AC}}$ is the formation constant of the complex $\mathrm{AC}$, and 
$\mu_{\mathrm{ep} . \mathrm{AC}}$ and $\mu_{\mathrm{ep}, \mathrm{A}}$ are the electrophoretic mobilities of the analyte-additive complex AC and the uncomplexed analyte, respectively. Clearly, eq 2.1 .5 is also analogous to eq 2.1.1.

The constants in eqs 2.1 .2 to 2.1 .5 are usually estimated by measuring the response at several ligand concentrations followed by one of several regression procedures. Although a nonlinear regression can be used to solve the constants directly, eq 2.1.1 is often linearized into one of the following equations:

$$
\begin{aligned}
& \frac{1}{y}=\frac{\mathrm{f}}{\mathrm{d}} \cdot \frac{1}{x}+\frac{\mathrm{e}}{\mathrm{d}} \\
& \frac{x}{y}=\frac{\mathrm{e}}{\mathrm{d}} x+\frac{\mathrm{f}}{\mathrm{d}} \\
& \frac{y}{x}=-\frac{\mathrm{e}}{\mathrm{f}} y+\frac{\mathrm{d}}{\mathrm{f}}
\end{aligned}
$$

The constants are estimated from the slopes and intercepts of straight lines ${ }^{(1)}$. The use of eqs 2.1.6 to 2.1.8 have acquired different names in different research fields but can be referred to most generally as double reciprocal (eq 2.1.6; also referred to as Lineweaver-Burk ${ }^{(15)}$ or BenesiHildebrand(2) plots), $y$-reciprocal (eq 2.1.7), and $x$-reciprocal (eq 2.1.8; also referred to as Eadie $^{(16)}$ or Scatchard(41) plots) methods. The linearizations of eq 2.1.5 are shown in Table 2.1.1.

It has long been realized that although the nonlinear regression and each of the three linearizations are based on the same equation, they often give different estimates and confidence intervals for the constants when applied to the same data $\operatorname{set}^{(42-45)}$. Linearizing eq 2.1 .1 to eqs 2.1.6 to 2.1.8 invalidates some of the assumptions made in performing the least squares regression analysis, including introducing error into the independent variable and transforming the error in the data to a non-Gaussian distribution ${ }^{(1)}$. The data spacing is changed when eq 2.2.1 is rearranged to eqs 2.1.6 and 2.1.8, which alters the weight on certain measurements. These problems can often be overcome if the data is weighted according to the functions listed in Table 
2.1.1(1). A description of how the weighting functions are derived is given in Appendix I.

Table 2.1.1 Nonlinear and linear forms of the binding isotherm in CE.

\begin{tabular}{|c|c|c|}
\hline $\begin{array}{l}\text { Calculation } \\
\text { Method }\end{array}$ & Equation & $\sigma_{y^{\prime}}^{2}+$ \\
\hline $\begin{array}{l}\text { Nonlinear } \\
\text { Regression }\end{array}$ & $\left(v \mu_{\theta p}^{A}-\mu_{e p, A}\right)=\frac{\left(\mu_{\theta p, A C}-\mu_{\theta p, A}\right) K_{A C}[C]}{1+K_{A C}[C]}$ & $\sigma_{y}^{2}$ \\
\hline \multirow{2}{*}{$\begin{array}{l}\text { Double } \\
\text { Reciprocal }\end{array}$} & 1 & $\sigma_{y}^{2}$ \\
\hline & $\overline{\left(v \mu_{\mathrm{ep}}^{\mathrm{A}}-\mu_{\mathrm{ep}, \mathrm{A}}\right)}=\overline{\left(\mu_{\mathrm{\theta p}, \mathrm{AC}}-\mu_{\theta \mathrm{e}, \mathrm{A}}\right) \mathrm{K}_{\mathrm{AC}}} \overline{[\mathrm{C}]}+\overline{\left(\mu_{\mathrm{ep}, \mathrm{AC}}-\mu_{\mathrm{ep}, \mathrm{A}}\right)}$ & $\left(v \mu_{\theta p}^{A}-\mu_{\theta p, A}\right)^{4}$ \\
\hline \multirow[t]{2}{*}{$y$-Reciprocal } & {$[\mathrm{C}]$} & {$[C]^{2} \sigma_{y}^{2}$} \\
\hline & $\overline{\left(v \mu_{\mathrm{ep}}^{\mathrm{A}}-\mu_{\mathrm{ep}, \mathrm{A}}\right)}=\overline{\left(\mu_{\mathrm{ep}, \mathrm{AC}}-\mu_{\mathrm{ep}, \mathrm{A}}\right)}+\overline{\left(\mu_{\mathrm{eP}, \mathrm{AC}}-\mu_{\mathrm{ep}, \mathrm{A}}\right) \mathrm{K}_{\mathrm{AC}}}$ & $\overline{\left(v \mu_{\theta P}^{A}-\mu_{\theta p, A}\right)^{4}}$ \\
\hline$x$-Reciprocal & $\frac{\left(\nu \mu_{\theta p}^{A}-\mu_{\theta p, A}\right)}{[C]}=-K_{A C}\left(v \mu_{\theta p}^{A}-\mu_{\theta p, A}\right)+K_{A C}\left(\mu_{\theta p, A C}-\mu_{\theta p, A}\right)$ & $\left(K_{A C}+\frac{1}{[C]}\right)^{2} \sigma_{y}^{2}$ \\
\hline
\end{tabular}

${ }^{+} \sigma_{y^{\prime}}^{2}$ is the variance of the transformed $y ; \sigma_{y}^{2}$ is the variance in $\left(v \mu_{e p}^{A}-\mu_{e p, A}\right)$; the weight for each point is equal to $1 / \sigma_{\mathrm{y}^{\prime}}^{2}$

Because of the complexity of the regression calculations, it is difficult to show how error is propagated through the different methods analytically. Dowd and Riggs ${ }^{(43)}$ first used Monte Carlo analyses to compare the different calculation methods and their estimates of the constants. Since then a number of researchers have used Monte Carlo analyses to simulate binding experiments ${ }^{(46-53)}$. It has been shown that the nonlinear regression method minimizes both the error and the bias in the estimates of the constants. Unfortunately, not enough conditions have been simulated to determine the effect experimental parameters have on the accuracy and precision of the estimated constants. The following sections describe our research into the effect the ligand concentration range and the maximum response range have on the estimated binding constant. 


\subsection{The Ligand Concentration Range}

\subsubsection{Introduction}

The range of ligand concentrations over which responses are measured has been identified as one of the most important aspects in the design of a binding experiment ${ }^{(1,44)}$. Intuitively, to minimize the error in the estimate of $\mathrm{K},[\mathrm{L}]$ should cover a significant portion of the possible response range with a number of points near the ligand concentration where half of the substrate is complexed. Theories do suggest that the error in the estimate of the equilibrium constant will be minimized when responses are measured over the central portion of the binding isotherm (i.e. the fraction of the analyte complexed should range from approximately 0.2 to 0.8)(44). Several Monte Carlo analyses have been made to determine the effect of the ligand concentration range on the estimate of the equilibrium constant ${ }^{(46,47,50)}$. Unfortunately, the effect experimental parameters have on the reliability of the estimated binding constants has not been studied thoroughly. Not enough data were collected to truly assess the theories proposed to describe the effect of ligand concentration on the error in binding constant estimates.

In this chapter, the effect of ligand concentration on the error in the estimate of $\mathrm{K}$ is studied more thoroughly. The nonlinear regression is compared with the three linearizations of the binding isotherm. The error and bias in the binding constant estimates is compared over a wide range of conditions, including cases where the fraction of substrate complexed is below 0.2 for all [L] or above 0.8 for all [L]. The terminology developed for CE (eq 2.1.5) will be used since this is the primary research interest of our group (i.e. analyte and additive are analogous to substrate and ligand, respectively). Also, the constants and errors in the data are typical for CE. It should be emphasized that although CE is used as an example in this chapter, the equations are analogous to those used in many other research areas, allowing the conclusions presented here to be applied to complexation chemistry in general. 


\subsubsection{Experimental}

Monte Carlo simulations of a dynamic complexation CE experiment were performed using a Visual Basic macro in Microsoft Excel 5.0 on a pentium PC. The simulations were made assuming the following: $\mu_{\mathrm{e}, \mathrm{A}}=2.5 \times 10^{-4} \mathrm{~cm}^{2} \mathrm{~V}^{-1} \mathrm{~s}^{-1} ; \mu_{\mathrm{ep}, \mathrm{AC}}=1.0 \times 10^{-4} \mathrm{~cm}^{2} \mathrm{~V}^{-1} \mathrm{~s}^{-1} ;$ separation potential $=$ $30 \mathrm{kV}$; total capillary length $=57 \mathrm{~cm}$; length to the detector $=50 \mathrm{~cm}$. Simulations were made for binding constants ranging from $1 \mathrm{M}^{-1}$ to $4000 \mathrm{M}^{-1}$ with a set range of additive concentrations. The additive concentrations used were $5,23.75,42.5,61.25$ and $80 \mathrm{mM}$. Equation 2.1.5 was used to calculate the true net analyte mobility at each additive concentration. The random number generator in Excel 5.0 was used to produce an experimental mobility according to a normal distribution which had a mean equal to the true mobility and a standard deviation of $8.75 \times 10^{-7} \mathrm{~cm}^{2} \mathrm{~V}^{-1} \mathrm{~s}^{-1}$. Two experimental mobilities were generated for each additive concentration. Four experimental mobilities were generated for an additive concentration of $0 \mathrm{mM}$, again with a standard deviation of $8.75 \times 10^{-7} \mathrm{~cm}^{2} \mathrm{~V}^{-1} \mathrm{~s}^{-1}$, in order to calculate $\mu_{\mathrm{ep}, \mathrm{A}}$. The experimental mobilities were then used to estimate the equilibrium constant according to one of the calculation methods. All regressions were made according to the least squares variance-covariance method. This procedure was repeated 1000 times for each of the four calculation methods at each K. Thirtyseven values for $\mathrm{K}$ were tested, giving rise to over 3.6 million simulated measurements, emphasizing the necessity of the computational approach used.

The effect of varying $\mathrm{K}$ for a set range of additive concentrations is shown in Figure 2.2.1. As $\mathrm{K}$ changes, so does the portion of the binding isotherm covered by the additive concentrations. At low values of the binding constant, the additive concentrations only cover the lower portion of the binding isotherm (i.e. the portion where the fraction of analyte complexed is low). Conversely, at high $\mathrm{K}$, the additive concentrations only cover the upper portion of the binding isotherm (i.e. the portion where the fraction of analyte complexed is high). Therefore, varying $\mathrm{K}$ for a set group of additive concentrations is analogous to scaling the additive 
100.

concentration range for a set $\mathrm{K}$. The absolute values of $\mathrm{K}$ and the additive concentrations are not as important as the portion of the binding isotherm that is covered considering both values. At $\mathrm{K}$ $=50 \mathrm{M}^{-1}$, the fraction of analyte complexed ranges from 0.2 to 0.8 for the additive concentrations used in this simulation. This has been previously suggested as the ideal range of additive concentrations ${ }^{(44)}$. At $\mathrm{K}=3.125 \mathrm{M}^{-1}$, the fraction of analyte complexed is equal to or below 0.2 for all of the additive concentrations. At $\mathrm{K}=800 \mathrm{M}^{-1}$, the fraction of analyte complexed is equal to or above 0.8 for all of the additive concentrations.

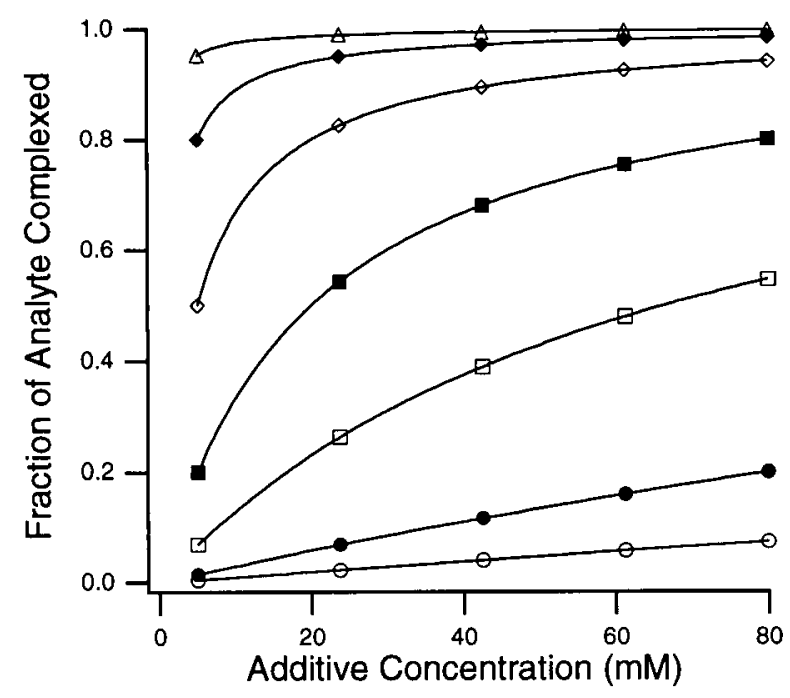

Figure 2.2.1 Binding isotherms for a set range of additive (ligand) concentrations at $\mathrm{K}=1 \mathrm{M}^{-1}(O), 3.125 \mathrm{M}^{-1}(\bullet), 15 \mathrm{M}^{-1}(\square), 50 \mathrm{M}^{-1}(\square)$, $200 \mathrm{M}^{-1}(\diamond), 800 \mathrm{M}^{-1}(\bullet)$, and $4000 \mathrm{M}^{-1}(\triangle)$.

\subsubsection{Error and Bias at Low Equilibrium Constants.}

Figure 2.2.2 depicts the distributions of the binding constants estimated using the different calculation methods for $\mathrm{K}=1$ to $50 \mathrm{M}^{-1}$. The markers represent the medians of the distributions. Medians were used because of their robustness and to minimize the influence of the grossly incorrect estimates that can occur when the additive concentrations do not cover the optimal range of the binding isotherm. The solid line indicates the true equilibrium constant. Therefore, deviation of the median from the solid line indicates bias in the calculation method. The dashed lines bound the range of the distribution that includes $95 \%$ of the binding constant 
101.

estimates (i.e. the $95 \%$ range). Therefore, $2.5 \%$ of the binding constant estimates were above the upper dashed line and $2.5 \%$ of the estimates were below the lower dashed line. The $95 \%$ range gives an indication of the precision of the calculation method. Obviously a narrow distribution of binding constant estimates is preferable.

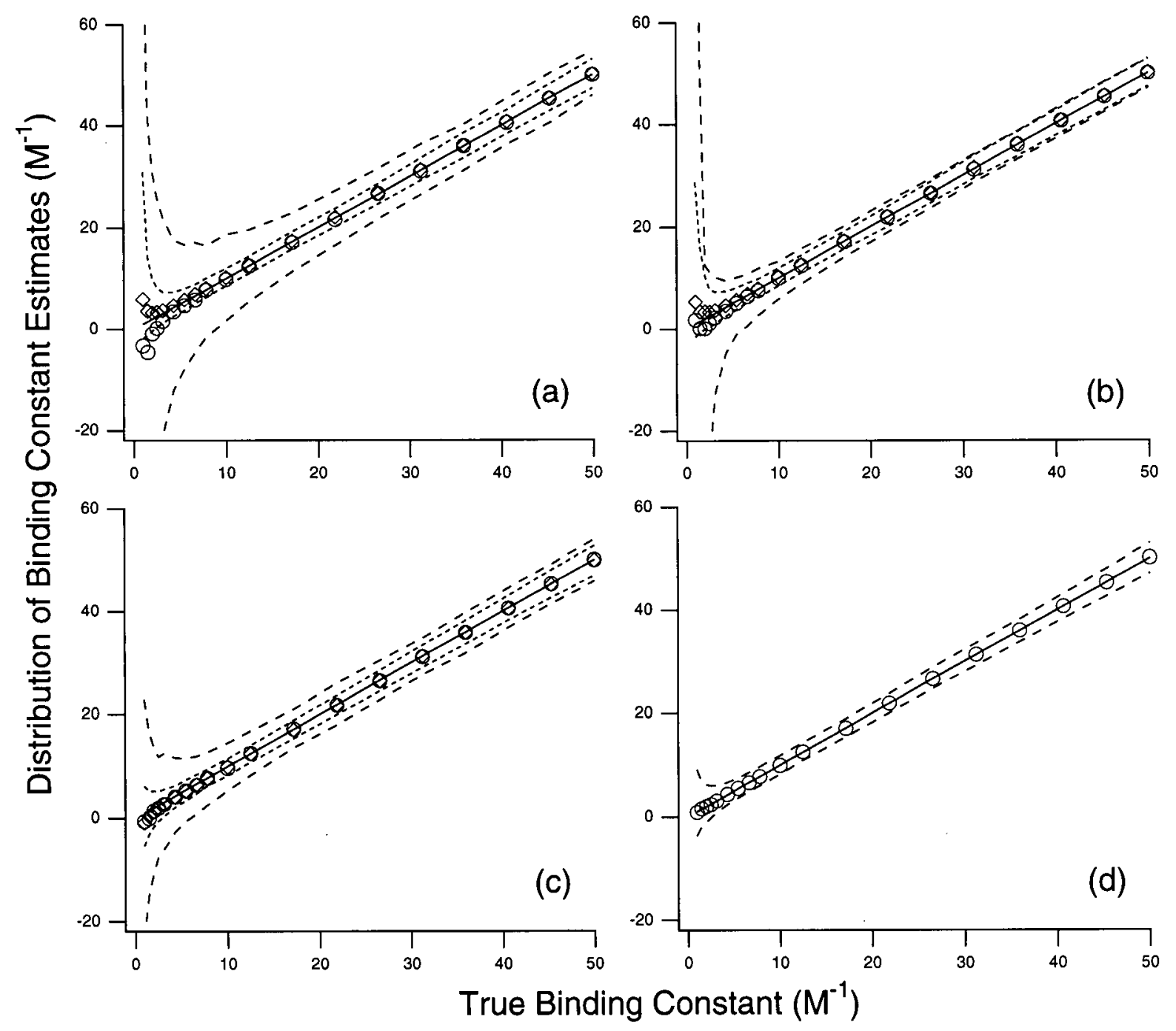

Figure 2.2.2 The distributions of the binding constant estimates calculated using (a) double reciprocal, (b) $y$-reciprocal, (c) $x$-reciprocal, and (d) nonlinear regression methods over $\mathrm{K}=1$ to $50 \mathrm{M}^{-1}$. The markers are the medians of the distributions for the weighted $(O)$ and unweighted $(\diamond)$ methods. The dashed lines define the $95 \%$ ranges of the distributions for the weighted $(--\cdot-\cdot)$ and unweighted $(---)$ methods.

Comparing the different calculation methods shows that the $95 \%$ range of the nonlinear regression method is generally narrower than that of the unweighted linear methods. In all three 
linearizations the distributions using weighted data are narrower than the unweighted for $\mathrm{K}=1$ to $50 \mathrm{M}^{-1}$. When ideally weighted, all of the calculation methods studied gave nearly identical 95\% ranges of the binding constant estimates. The weighted and unweighted nonlinear regressions are identical since no weighting is required when the errors in the data points are equal. The elimination of the weighting procedure is a major advantage of the nonlinear regression method. It should be emphasized that because the errors in this experiment were simulated, it was possible to calculate the exact weighting functions for each method. In practical applications, the error at each point is usually estimated, which may give rise to inaccurate weighting functions. The $95 \%$ ranges presented for the weighted methods therefore represent the minimum range possible with ideal weighting functions. The nonlinear regression method is the least dependent on the choice of the weighting function and should provide the most reliable binding constant estimate in practical applications.

As shown in Figure 2.2.1, at $\mathrm{K}=50 \mathrm{M}^{-1}$, the fraction of analyte complexed ranges from 0.2 to 0.8 . It has been previously suggested that this is the range of additive concentrations that minimizes the error in the estimated binding constant ${ }^{(44)}$. As the binding constant decreases from $50 \mathrm{M}^{-1}$, the fraction of analyte complexed at some of the additive concentrations drops below the 0.2 threshold. When $\mathrm{K}$ is below $3.125 \mathrm{M}^{-1}$, the fraction of analyte complexed is less than 0.2 for all of the additive concentrations used.

At low binding constants, where the fraction of analyte complexed is below 0.2 , there is a significant increase in the $95 \%$ range of the $\mathrm{K}$ estimates, especially when the three linear methods are used. When the additive concentrations only cover the lower portion of the binding isotherm it is impossible to accurately determine the value of $\mathrm{K}$. In some cases, a substantial portion of the estimates was negative. At low $\mathrm{K}$ values, the distributions of binding constant estimates are no longer symmetrical around the median. This may cause problems when calculating the confidence intervals for the estimated binding constants. 
Bias becomes significant in the three linearization methods when the binding constant is low. In some cases, the median of the distribution was negative, indicating that the method gave more negative estimates for $\mathrm{K}$ than positive estimates. Weighting did not remove the bias from the binding constant estimates. Bias is a serious problem since it cannot be eliminated through replicate experiments. In binding experiments though, the amount of bias present at low $K$ is small in relation to the $95 \%$ ranges of the distributions, suggesting that bias is not the most critical problem in this case. No bias was detected when the nonlinear method was used to estimate the equilibrium constant, further supporting the premise that the nonlinear regression method gives more reliable binding constant estimates than the three linearizations.

\subsubsection{Error and Bias at High Equilibrium Constants.}

Figure 2.2.3 shows the distributions of the binding constant estimates for $\mathrm{K}=50$ to 4000 $\mathrm{M}^{-1}$. At $\mathrm{K}=50 \mathrm{M}^{-1}$ the fraction of analyte complexed ranges from 0.2 to 0.8 . When $\mathrm{K}$ is higher than $800 \mathrm{M}^{-1}$, the fraction of analyte complexed is equal to or greater than 0.8 at all of the additive concentrations (e.g. the fraction of analyte complexed ranges from 0.952 to 0.997 for the additive concentrations used in these simulations when $K=4000 \mathrm{M}^{-1}$ ). At higher $\mathrm{K}$ values, when the fraction of analyte complexed is above 0.8 , the $95 \%$ ranges of the binding constant estimates increase. With the exception of the $y$-reciprocal plots, the ranges of the binding constant estimates for the nonlinear, double reciprocal and $x$-reciprocal methods are nearly identical, and weighting does not seem to have any effect. The precision of the unweighted $y$-reciprocal method is significantly poorer than the other methods at higher $\mathrm{K}$ values. When ideally weighted though, the $95 \%$ ranges of the $y$-reciprocal binding constant estimates are comparable to the other methods. Again, it was possible to ideally weight the data in the simulated experiments, but would be difficult to do so in practical cases. Therefore, when the additive concentrations only cover the upper portion of the binding isotherm, it would be more reasonable to use one of 
104.

the methods that are less dependent on choosing the proper weighting function (i.e. nonlinear, double reciprocal or $x$-reciprocal).

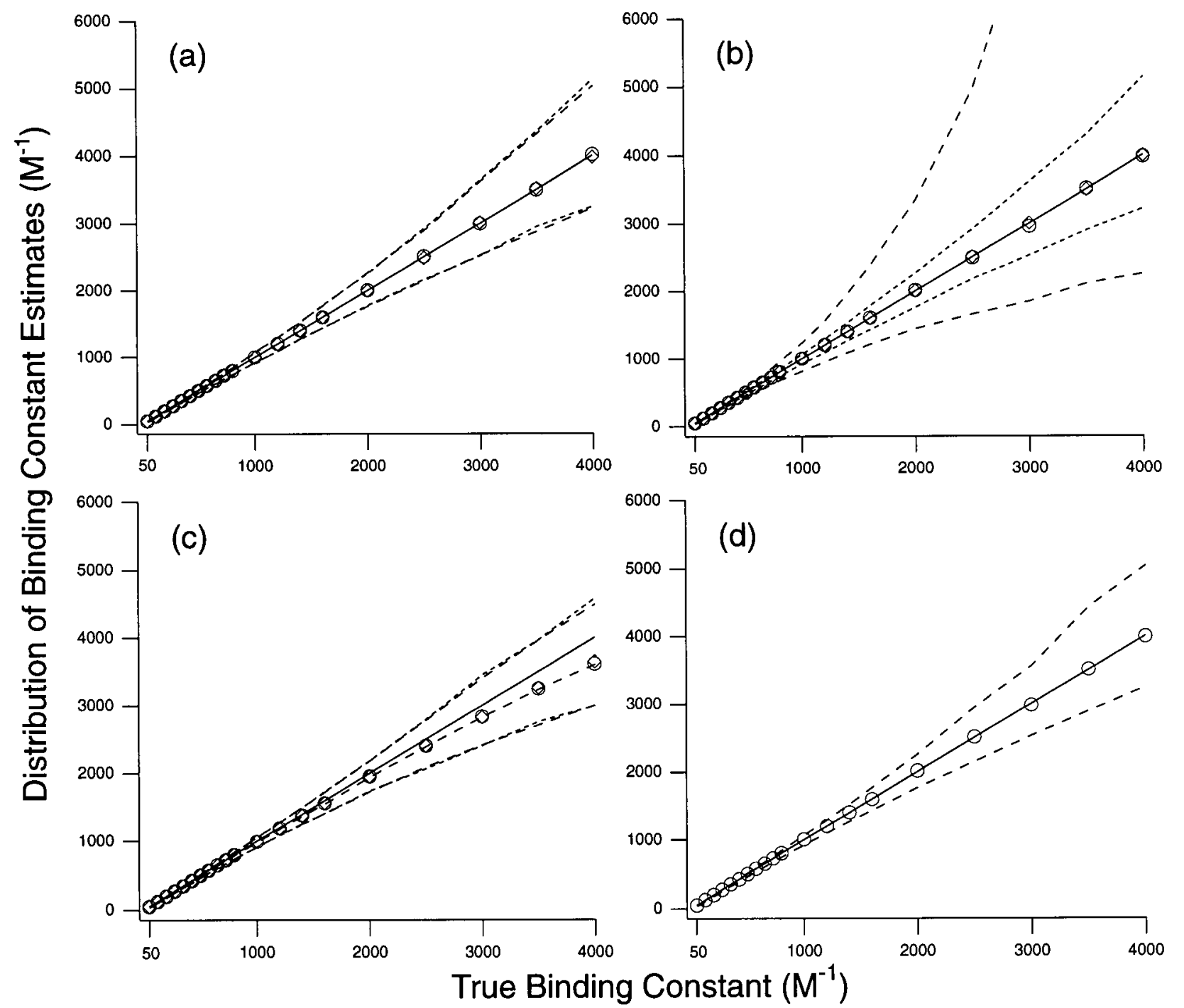

Figure 2.2.3 The distributions of the binding constant estimates calculated using (a) double reciprocal, (b) $y$-reciprocal, (c) $x$-reciprocal, and (d) nonlinear regression methods over $K=50$ to $4000 \mathrm{M}^{-1}$. The symbols are the same as in Figure 2.2.2.

Bias is significant in the $x$-reciprocal plot at higher $\mathrm{K}$ values and weighting the data does not alleviate the problem. Bias is troublesome since it cannot be eliminated through replicate experiments. Therefore, the $x$-reciprocal plot is unsuitable for estimating the binding constant when the fraction of analyte complexed is high. 


\subsubsection{Comparing the Relative 95\% Ranges at Low K and High K}

Comparing the distributions reveals that the relative error in the binding constant estimates does not increase as quickly at high $\mathrm{K}$ as it does for low $\mathrm{K}$. At low $\mathrm{K}$ the $95 \%$ range increases quickly, making it impossible to obtain accurate binding constant estimates. This does not occur at high $\mathrm{K}$, even when the binding constant is increased to $4000 \mathrm{M}^{-1}$. Figure 2.2 .4 shows that the relative $95 \%$ ranges of the $\mathrm{K}$ estimates (i.e. the $95 \%$ range divided by the true value of $\mathrm{K}$ ) at low binding constants are much higher that those at high $\mathrm{K}$.

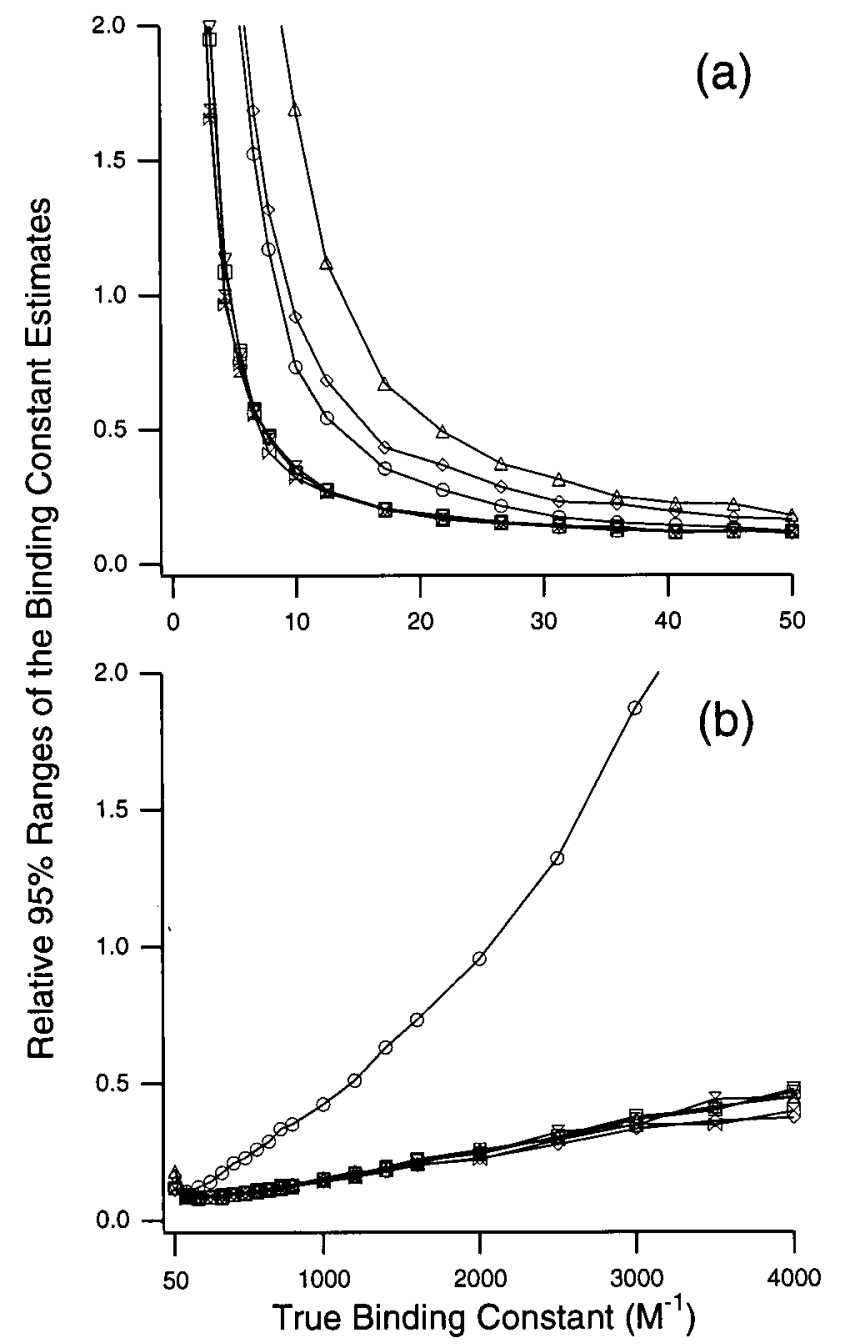

Figure 2.2.4 The relative 95\% ranges of the binding constants estimated using the double reciprocal $(\triangle)$, weighted double reciprocal $(\square), \quad y$-reciprocal $(O)$, weighted $y$-reciprocal $(\nabla), x$-reciprocal $(\diamond)$, weighted $x$-reciprocal $(\bowtie)$, and nonlinear regression methods $(\nabla)$ for $(a)$ $\mathrm{K}=1$ to $50 \mathrm{M}^{-1}$, and (b) $\mathrm{K}=50$ to $4000 \mathrm{M}^{-1}$.

The relative $95 \%$ range using the nonlinear regression at $\mathrm{K}=3.125 \mathrm{M}^{-1}$ (fraction of 
analyte complexed ranges from 0.015 to 0.2 ) is approximately 14 times that when $\mathrm{K}=800 \mathrm{M}^{-1}$ (fraction of analyte complexed ranges from 0.8 to 0.985 ), even though they both cover similar amounts of the binding isotherm (i.e. the difference in the fraction of analyte complexed at the lowest and highest additive concentration is the same for both). Even at $K=4000 \mathrm{M}^{-1}$ the relative $95 \%$ range is approximately one quarter of that when $\mathrm{K}=3.125 \mathrm{M}^{-1}$ even though the fraction of analyte complexed only ranges from 0.952 to 0.997 . This contrasts what is suggested by some theories that the error contributed by the upper and lower portion of the binding isotherm should be symmetrical around the point where half of the analyte is complexed ${ }^{(44)}$.

\subsubsection{Semi-Empirical Prediction of the 95\% Range}

An important goal of Monte Carlo simulations should be to determine the optimum conditions for actual experiments. It would be useful to predict the effect of the additive concentration range on the reliability of the estimated binding constant. Several researchers have proposed methods to assess the reliability of binding constants estimated using a certain additive concentration range. Deranleau ${ }^{(44)}$ suggested that propagation of error could be used to estimate the relative error in the calculated binding constant:

$$
\frac{\Delta \mathrm{K}}{\mathrm{K}} \geq \Delta \mathrm{s} \sqrt{\frac{1}{\mathrm{~s}^{2}}+\frac{1}{(1-\mathrm{s})^{2}}}
$$

where $\Delta \mathrm{K}$ is the error in the binding constant $\mathrm{K}, \mathrm{s}$ is the fraction of analyte complexed, and $\Delta \mathrm{s}$ is the error in the fraction of analyte complexed. According to eq 2.2.1, the error contributed by the lower and upper portions of the binding isotherm should be symmetrical around the point where half of the analyte is complexed, suggesting that the relative error in the estimated binding constant will be minimized when data are collected between $s=0.2-0.8$. Unfortunately, this method only considers the error contributed by a single point, not a range of additive concentrations. Differences in the shape of the lower and upper portions of the binding isotherm are also not considered. 
Weber and Anderson ${ }^{(54)}$ suggested that the information content of the measured responses should be maximized to ensure the reliability of the estimated equilibrium constant. The information content of a single measurement $(\mathrm{I}(\mathrm{p}))$ is given by:

$$
I(p)=-p \log _{2} p-(1-p) \log _{2}(1-p)
$$

where $\mathrm{p}$ is the fraction of analyte complexed. The amount of information provided by a number of measurements can be found by summing the information content of the individual measurements. Although this approach accounts for a range of additive concentrations, the data are still considered individually. The shape of the curve is still not accounted for.

Carta et al. ${ }^{(55,56)}$ proposed that reliable estimates for the equilibrium constant can be obtained when additive concentrations with a wide range of $G$ values are tested. $G$ represents the fraction of uncomplexed analyte and is given by:

$$
G=\frac{1}{K \sqrt{\left(a+b+K^{-1}\right)^{2}-4 a b}}
$$

where $\mathrm{a}$ and $\mathrm{b}$ are the concentrations of the analyte (substrate) and the additive (ligand). When the concentration of the additive is much greater than that of the analyte (common in most $\mathrm{CE}$ binding studies), eq 2.2.3 simplifies to:

$$
\mathrm{G}=\frac{1}{1+\mathrm{Kb}}
$$

which is equal to the fraction of analyte uncomplexed at additive concentration $\mathrm{b}$. The error in $\mathrm{K}$ should be minimized by maximizing:

$$
\sum_{i=1}^{n}\left(G_{i}-G_{\text {mean }}\right)^{2}
$$

where $G_{\text {mean }}$ is the average value of $G$ and $n$ is the number of measurements. This gives a measure of the range of the binding isotherm covered by the additive concentrations and is 
approximately symmetrical around the point where half of the analyte is complexed.

Unfortunately, none of the theories proposed above accurately explain the trends observed in this experiment. Fig 2.2.5 shows the dispersions of $\mathrm{G}$ values and the information contents of the experiments simulated in this chapter. The error in the estimate of $\mathrm{K}$ should be minimized at maximum values of $\Sigma\left(G_{i}-G_{\text {mean }}\right)^{2}$ and $\Sigma I(p)$. The dispersion of $G$ is greatest when $\mathrm{K}=50 \mathrm{M}^{-1}$, where data is collected symmetrically around the point where half of the analyte is complexed. Also, the method proposed by Carta et al. suggests that there should be similar errors in the binding constant estimates at $\mathrm{K}=3.125 \mathrm{M}^{-1}$ and $\mathrm{K}=800 \mathrm{M}^{-1}$. This does not agree with Figure 2.2.4 in which the minimum relative 95\% range of the binding constant estimates is at $\mathrm{K}=200 \mathrm{M}^{-1}$ and substantially narrower ranges are achieved when the additive concentrations cover the upper portion of the binding isotherm (i.e. where the fraction of analyte complexed is greater than 0.5 ). Information theory (Figure 2.2.5(b))does not explain the trends in Figure 2.2.4 either. Information theory predicts that the error in the estimate of $\mathrm{K}$ will be minimized at $\mathrm{K}=$ $25 \mathrm{M}^{-1}$, and that the error in $\mathrm{K}$ will generally be lower when the additive concentrations cover the lower portion of the binding isotherm.

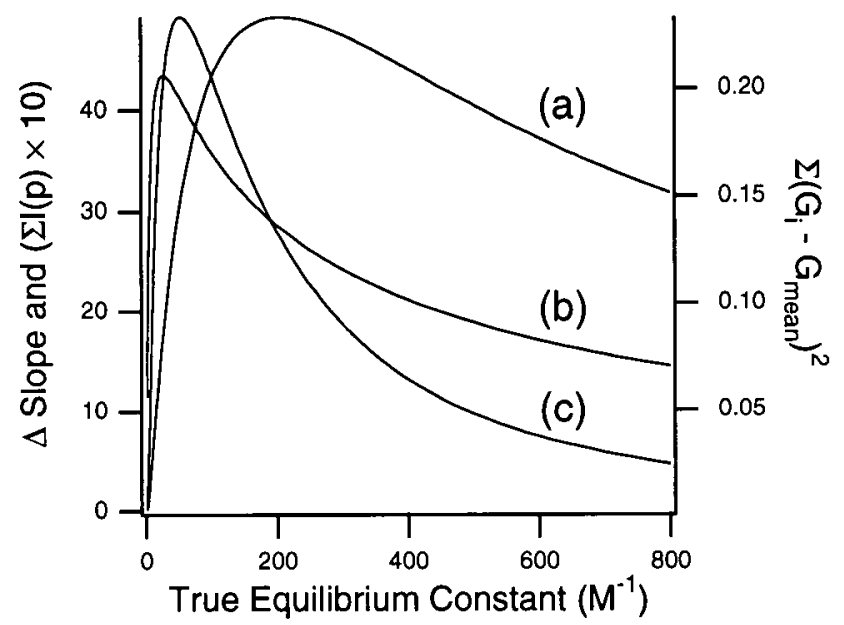

Figure 2.2.5 The (a) change in slope, (b) information content, and (c) dispersion of $\mathrm{G}$ for binding isotherms with $\mathrm{K}=1$ to $800 \mathrm{M}^{-1}$ and a set range of additive (ligand) concentrations $(5,23.75,42.5,61.25$, and 80 $\mathrm{mM})$. 
109.

Previous explanations of the effect of the additive concentration range focused on the amount of error contributed by individual data points, but did not account for the geometric properties of the binding isotherm. In a rectangular hyperbola (eqs 2.1 .2 to 2.1.5), the equilibrium constant dictates the amount of curvature in the isotherm. Therefore, it is impossible to make an accurate estimate of $\mathrm{K}$ when there is no observable curvature in the isotherm. Using this approach, it is clear that better estimates for $\mathrm{K}$ can be achieved when the additive concentrations cover the upper portion of the binding isotherm. Comparing the isotherms for $\mathrm{K}$ $=3.125 \mathrm{M}^{-1}$ and $\mathrm{K}=800 \mathrm{M}^{-1}$ in Figure 2.2.1 reveals that even though both curves cover a similar amount of the binding isotherm (analyte complexed ranging from 0.015 to 0.2 for $\mathrm{K}=3.125 \mathrm{M}^{-1}$ and 0.8 to 0.985 for $\mathrm{K}=800 \mathrm{M}^{-1}$ ), there is obviously more curvature in the plot for $\mathrm{K}=800 \mathrm{M}^{-1}$ than in that for $\mathrm{K}=3.125 \mathrm{M}^{-1}$.

The amount of curvature in a binding isotherm can be estimated by finding the difference between the slope at the lowest additive concentration and that at the highest concentration. The slope at any point on the curve is equal to the first derivative of the response with respect to the additive concentration. For example, the first derivative of eq 2.1.5 is:

$\frac{\partial\left(v \mu_{\mathrm{ep}}^{\mathrm{A}}\right)}{\partial[\mathrm{C}]}=\frac{\left(\mu_{\mathrm{ep}, \mathrm{AC}}-\mu_{\mathrm{ep}, \mathrm{A}}\right) \mathrm{K}_{\mathrm{AC}}}{\left(1+\mathrm{K}_{\mathrm{AC}}[\mathrm{C}]\right)^{2}}$

Figure 2.2.5(a) shows the difference in the slope of the lowest and highest additive concentration over $\mathrm{K}=0$ to $800 \mathrm{M}^{-1}$. The change in slope explains the trends of the relative $95 \%$ range presented in this chapter remarkably well. The maximum difference in the slope occurs at approximately $\mathrm{K}=200 \mathrm{M}^{-1}$, in agreement with the minimum relative $95 \%$ range observed in Figure 2.2.4. Also, the difference in the slope is significantly larger when the data covers the upper portion of the isotherm, again in agreement with Figure 2.2.4. 
110.
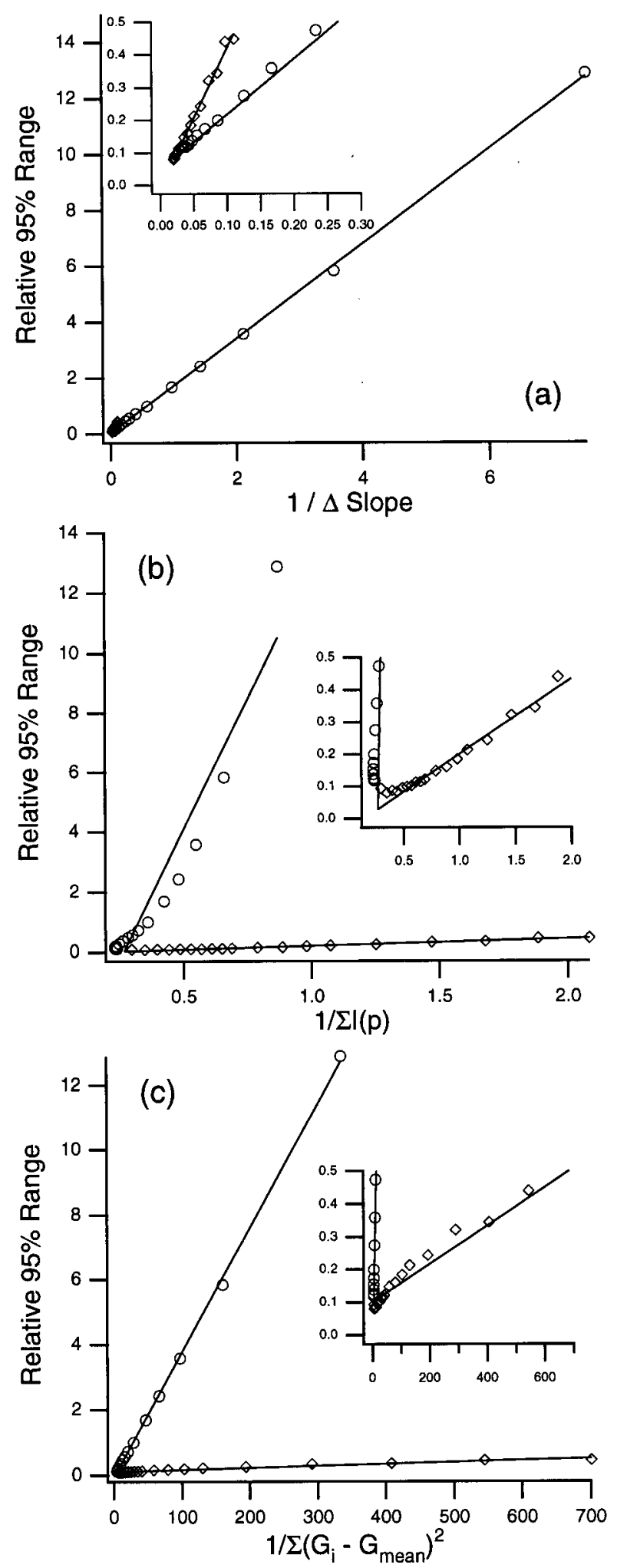

Figure 2.2.6 Correlations between the relative 95\% ranges of the binding constants estimated using the nonlinear regression method and (a) $(\Delta \text { slope })^{-1}$, (b) $(\Sigma \mid(p))^{-1}$, and $(c)\left(\Sigma\left(G_{i}-G_{\text {mean }}\right)^{2}\right)^{-1}$ for $K=1$ to $50 M^{-1}(O)$ and $K=50$ to $4000 \mathrm{M}^{-1}(\diamond)$. 
Figure 2.2.6 shows the correlation between the relative $95 \%$ range of binding constant estimates vs. the reciprocal of the difference in slopes, the information content, and the dispersion in G. There appears to be an excellent correlation between the relative $95 \%$ range and the reciprocal of the change in slope. However, closer inspection reveals two lines: one when the additive concentrations cover the lower portion of the isotherm and one when the additive concentrations cover the upper portion. The two distinct correlations indicate that the error introduced by the upper and lower portion of the binding isotherm propagate to the error in the binding constant estimates differently. The difference may be caused by the data spacing which is not accounted for in the change in slope. When the data is evenly spaced, and the additive concentrations only cover the upper portion of the isotherm, the curvature in the plot is concentrated over several of the points, putting undue emphasis on these points. This may account for the larger relative $95 \%$ ranges of binding constant estimates than predicted by the inverse of the difference in slope. When the additive concentrations cover the lower portion of the isotherm, the curvature is more evenly spaced over the data set giving rise to a narrower range of binding constant estimates than would be predicted by the change in slope.

Regardless of the two distinct correlations, the reciprocal of the change in slope is a useful indicator of the significance of the estimated equilibrium constant. It correctly determines the conditions that minimize the relative $95 \%$ range of $\mathrm{K}$ estimates. Also the inverse change in slope is linearly correlated with the relative $95 \%$ range of equilibrium constant estimates over a wide range of conditions.

$1 / \Sigma \mathrm{I}(\mathrm{p})$ (Figure 2.2.6(b)) and $1 / \Sigma\left(\mathrm{G}_{\mathrm{i}}-\mathrm{G}_{\text {mean }}\right)^{2}$ (Figure 2.2.6(c)) do not correlate well with the relative $95 \%$ range of binding constant estimates. There are two distinct correlations for both the information content and dispersion in $G$ and the difference in the slopes of the two correlations is much greater for the information content and the dispersion in $\mathrm{G}$ than that of the change in slope. Also, the correlations are not all linear. There is no well-defined intersection in 
the correlations for the information content and the dispersion in $\mathrm{G}$. The rounded intersections of the correlations indicate that the information content and the dispersion in $\mathrm{G}$ do not accurately predict the minimum relative $95 \%$ range (i.e. the minimum relative $95 \%$ range and the minimum $1 / \Sigma \mathrm{I}(\mathrm{p})$ and $1 / \Sigma\left(\mathrm{G}_{\mathrm{i}}-\mathrm{G}_{\text {mean }}\right)^{2}$ do not occur at the same points), and are not suitable for predicting the optimum experimental conditions.

\subsubsection{Correlation Coefficient.}

The correlation coefficient $\left(\mathrm{R}^{2}\right)$ is probably the most commonly quoted indicator of how well a data set fits a particular mathematical model, even though high $\mathrm{R}^{2}$ values are sometimes obtained even when the data does not fit the model. For this reason, visual inspections of the data plots should always be used to confirm the applicability of a model to a certain data set. Nonetheless, because it is impractical to visually inspect the many thousands of experiments simulated in this experiment, the correlation coefficient was used to compare how well the quality of the data was reflected by the various calculation methods.

Figure 2.2.7 shows the median correlation coefficients for the different calculation methods over the range of binding constants tested. At low $\mathrm{K}$, where the additive concentrations only cover the lower portion of the binding isotherm, the $\mathrm{R}^{2}$ for the $x$-reciprocal and $y$-reciprocal plots decrease significantly, reflecting the unreliable nature of the $\mathrm{K}$ estimates under these conditions. Weighting the data increases $\mathrm{R}^{2}$ for the $x$-reciprocal and $y$-reciprocal plots but this is in line with the narrower range of binding constant estimates obtained through weighting. The correlation coefficient is a very poor measure of the accuracy when the double reciprocal plot is used to estimate binding constants when the additive concentrations only cover the lower portion of the binding isotherm. Very high $\mathrm{R}^{2}$ values are obtained even when the relative $95 \%$ range of $\mathrm{K}$ estimates is high. This is misleading since it suggests that the data fits the model very well even though it is impossible to make valid estimates for $\mathrm{K}$. The problem is more severe with 
weighted double reciprocal plots where the median $R^{2}$ is almost one even when $K=1 M^{-1}$. The nonlinear fitting method also gives high $\mathrm{R}^{2}$ values at low $\mathrm{K}$.

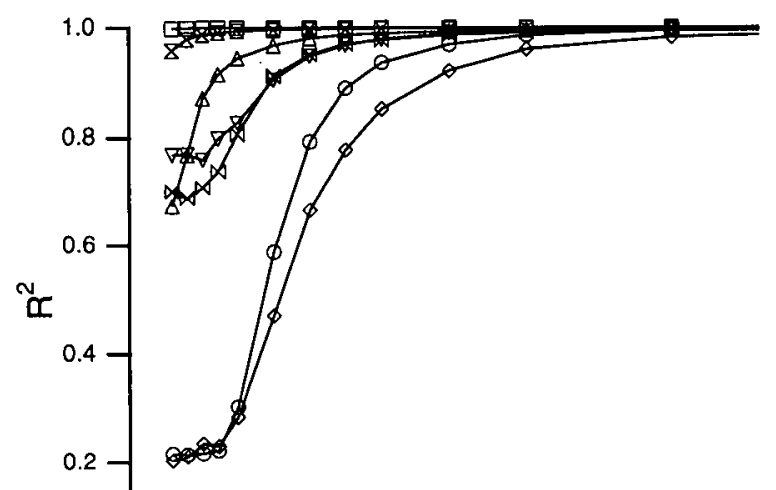

(a)

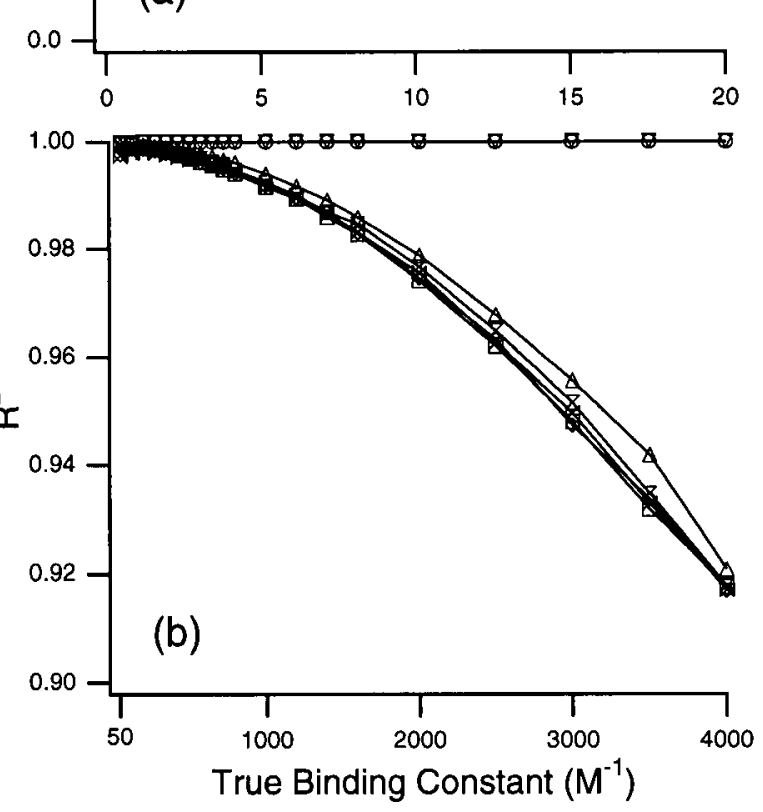

Figure 2.2.7 The median correlation coefficients $\left(R^{2}\right)$ for the double reciprocal $(\triangle)$, weighted double reciprocal $(\square), y$-reciprocal $(O)$, weighted $y$-reciprocal $(\nabla), x$-reciprocal $(\diamond)$, weighted $x$-reciprocal $(\bowtie)$, and nonlinear regression methods $(\nabla)$ for $(a) K=1$ to $20 \mathrm{M}^{-1}$, and (b) $\mathrm{K}=$ 50 to $4000 \mathrm{M}^{-1}$.

The decrease in $\mathrm{R}^{2}$ at high $\mathrm{K}$ is less pronounced than that observed at low $\mathrm{K}$. The smaller decrease in $\mathrm{R}^{2}$ is expected since the calculation methods generally provide better binding constant estimates when the additive concentrations cover the upper portion of the binding isotherm. With the exception of the $y$-reciprocal plots, the calculation methods all give similar 
$\mathrm{R}^{2}$ at high $\mathrm{K}$. The $\mathrm{R}^{2}$ for the $y$-reciprocal plots is a poor indicator of the reliability of the estimated binding constant. Even at $\mathrm{K}=4000 \mathrm{M}^{\mathrm{i}}$, the median $\mathrm{R}^{2}$ for the $\mathrm{y}$-reciprocal plots is almost one. Because $x$-reciprocal plots give the most realistic indication of the ability of a data set to provide reliable estimates of the binding constant at both low $\mathrm{K}$ and high $\mathrm{K}$, this method should be used to assess the quality of the experimental data.

\subsubsection{Conclusions}

All of the calculation methods gave similar ranges of binding constant estimates when the ideal weighting functions were used. However, because it is difficult to obtain the weighting function accurately in practical applications, we recommend using the nonlinear regression method because the weighted and unweighted regression give similar results, making the estimated binding constant less dependent on the choice of the weighting function.

This chapter demonstrated the importance of choosing an appropriate range of additive (ligand) concentrations when measuring a binding constant. The binding isotherm is determined by three parameters: the response of the free and complexed analyte establish the minimum and maximum values of the isotherm, and the binding constant determines the curvature of the isotherm. In most cases the properties of the uncomplexed analyte can be accurately determined. Therefore, the accuracy of $\mathrm{K}$ is often dictated by the accuracy of the measurement of the maximum response and the curvature of the isotherm. As can be seen in Figure 2.2.1, at low $\mathrm{K}$ values, neither the maximum response nor the curvature of the isotherm can be accurately determined, leading to high uncertainty in the binding constant estimates. At high $\mathrm{K}$ values, the complex mobility can be accurately determined and there is more curvature observed in the isotherm, giving rise to more accurate estimates of the binding constant.

The optimal additive concentration range is related to the strength of the interaction under 
investigation. Although in this simulation $\mathrm{K}$ was adjusted for a set range of additive concentrations, in practical experiments it is necessary to adjust the additive concentration range to match the binding constant. It was shown that the additive concentrations should cover the central to upper portion of the binding isotherm (i.e. where the fraction of analyte complexed is above 0.5 ). At higher $\mathrm{K}$, the additive concentrations should be low so the fraction of analyte complexed falls within the ideal range. Similarly, at higher $\mathrm{K}$, lower additive concentrations need to be used. In practical applications, a preliminary experiment should be performed to provide a rough estimate of the binding constant. The preliminary estimate of $\mathrm{K}$ can then be used to determine the range of additive concentrations which maximizes the difference in the slope of the isotherm, thereby minimizing the error in the final estimate of the binding constant. 


\subsection{The Maximum Response Range}

\subsubsection{Introduction}

The shape of a rectangular hyperbola (eq 2.1.1) is determined by two constants: the binding constant and the maximum response range. As shown in the previous chapter, the magnitude of the binding constant must be considered when choosing the ligand concentration range in order to obtain reliable estimates of the binding constant. Another concern is the effect of the maximum response range (i.e. the difference between the response at zero and infinite ligand concentrations). As shown in Figure 2.3.1, if the maximum response range is zero, the binding isotherm takes the shape of a straight line, making it impossible to estimate the binding constant. Table 2.3.1 lists the equations that describe substrate-ligand interactions (with 1:1 stoichiometries) and the specific constants that determine the maximum response range in UVvis absorption, NMR, Michaelis-Menten kinetics, and CE.

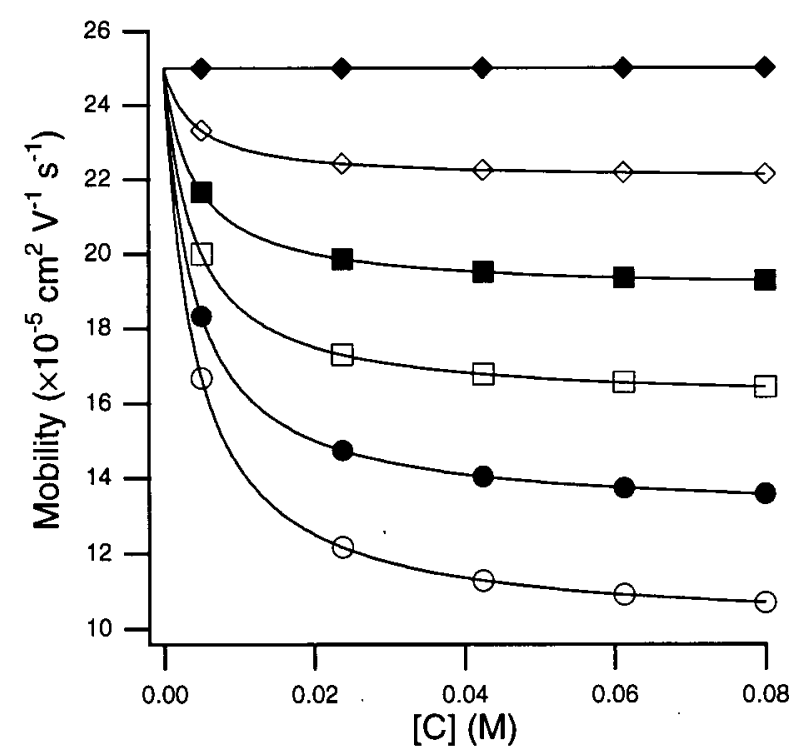

Figure 2.3.1 Binding isotherms with different maximum response ranges. The constants used to generate the curves are: $\mathrm{K}=250 \mathrm{M}^{-1} ; \mu_{\mathrm{ep.A}}$ $=2.5 \times 10^{-4} \mathrm{~cm}^{2} \mathrm{~V}^{-1} \mathrm{~s}^{-1}$; and $\mu_{\text {ep.AC }}=2.5(\bullet), 2.2(\diamond), 1.9(\square), 1.6(\square), 1.3$ $(\bullet)$, and $1.0 \times 10^{-4} \mathrm{~cm}^{2} V^{-1} \mathrm{~s}^{-1}(\mathrm{OP}, \mathrm{AC})$. 
Table 2.3.1 Equations and maximum response ranges for absorbance, NMR, Michaelis-Menten kinetics and capillary electrophoresis

\begin{tabular}{|c|c|c|}
\hline Technique & Equation & Maximum Response Range \\
\hline Absorbance & $\frac{\left(A-A_{S}\right)}{b}=\frac{\left[S_{0}\right]\left(\varepsilon_{S L}-\varepsilon_{S}\right) K[L]}{1+K[L]}$ & $\mathrm{b}\left[\mathrm{S}_{0}\right]\left(\varepsilon_{\mathrm{SL}}-\varepsilon_{\mathrm{S}}\right)$ \\
\hline NMR & $\left(\delta-\delta_{S}\right)=\frac{\left(\delta_{S L}-\delta_{S}\right) K[L]}{1+K[L]}$ & $\left(\delta_{S L}-\delta_{S}\right)$ \\
\hline $\begin{array}{l}\text { Michaelis-Menten } \\
\text { Kinetics }\end{array}$ & $V=\frac{V_{m}[S]}{K_{m}+[S]}$ & $V_{m}$ \\
\hline $\begin{array}{l}\text { Capillary } \\
\text { Electrophoresis }\end{array}$ & $\left(v \mu_{\theta p}^{A}-\mu_{\theta p, A}\right)=\frac{\left(\mu_{\theta p, A C}-\mu_{e p, A}\right) K_{A C}[C]}{1+K_{A C}[C]}$ & $\left(\mu_{\theta p, A C}-\mu_{\theta P, A}\right)$ \\
\hline
\end{tabular}

In this chapter, the effect of the maximum response range on the accuracy and precision estimated binding constants is studied. Nonlinear regression is compared with the three linearizations of the binding isotherm. Again, terminology developed for CE (eq 2.1.5) will be used since this is the primary research interest of our group (i.e. analyte and additive are analogous to substrate and ligand, respectively). Therefore, the constants and errors in the data are typical for CE. It should be emphasized that although $\mathrm{CE}$ is used as an example in this chapter, the equations are analogous to those used in many other research areas (see Table 2.3.1), allowing the conclusions presented here to be applied to complexation chemistry in general.

\subsubsection{Experimental}

Monte Carlo simulations of a dynamic complexation CE experiment were performed using a Visual Basic macro in Microsoft Excel 5.0 on a pentium PC. The simulations were made assuming the following: $\mu_{\mathrm{e}, \mathrm{A}}=2.5 \times 10^{-4} \mathrm{~cm}^{2} \mathrm{~V}^{-1} \mathrm{~s}^{-1} ; \mathrm{K}=250 \mathrm{M}^{-1}$; separation potential $=-30 \mathrm{kV}$; total capillary length $=57 \mathrm{~cm}$; length to the detector $=50 \mathrm{~cm}$. The additive concentrations used to perform the simulations were $5,23.75,42.5,61.25$ and $80 \mathrm{mM}$ which were shown chapter 2.2 to approximately cover the optimum concentration range for $\mathrm{K}=250 \mathrm{M}^{-1}$. Simulations were made 
118.

for $\mu_{\mathrm{ep}, \mathrm{AC}}$ ranging from $-1 \times 10^{-4} \mathrm{~cm}^{2} \mathrm{~V}^{-1} \mathrm{~s}^{-1}$ to $-2.5 \times 10^{-4} \mathrm{~cm}^{2} \mathrm{~V}^{-1} \mathrm{~s}^{-1}$. Equation 2.1 .5 was used to calculate the true net analyte mobility at each additive concentration. The random number generator in Excel 5.0 was used to produce an experimental mobility according to a normal distribution which had a mean equal to the true mobility and a standard deviation of $8.75 \times 10^{-7}$ $\mathrm{cm}^{2} \mathrm{~V}^{-1} \mathrm{~s}^{-1}$. Two experimental mobilities were generated for each additive concentration. Four experimental mobilities were generated for an additive concentration of $0 \mathrm{mM}$, again with a standard deviation of $8.75 \times 10^{-7} \mathrm{~cm}^{2} \mathrm{~V}^{-1} \mathrm{~s}^{-1}$, in order to calculate $\mu_{\mathrm{ep}, \mathrm{A}}$. The experimental mobilities were then used to estimate the equilibrium constant according to one of the four calculation methods. All regressions were made according to the least squares variance-covariance method. This procedure was repeated 1000 times for each calculation method at each $\mu_{\mathrm{ep}, \mathrm{AC}}$. Twenty-three values for $\mu_{\mathrm{ep}, \mathrm{AC}}$ were tested, giving rise to over 2.25 million simulated measurements, emphasizing the necessity of the computational approach used.

\subsubsection{Distribution of Binding Constant Estimates.}

Figure 2.3.2 shows the distributions of the binding constants estimated using the nonlinear regression and the three linear transformations. The markers represent the medians of the distributions. Medians were chosen to represent the central tendency of the distributions because of their robustness and insensitivity to the grossly inaccurate estimates that sometimes occur when experiments are performed under non-ideal conditions. The dashed lines bound the range of the distribution that includes $95 \%$ of the binding constant estimates (i.e. the $95 \%$ range). Therefore, $2.5 \%$ of the binding constant estimates were above the upper dashed line and $2.5 \%$ of the estimates were below the lower dashed line. 
119.

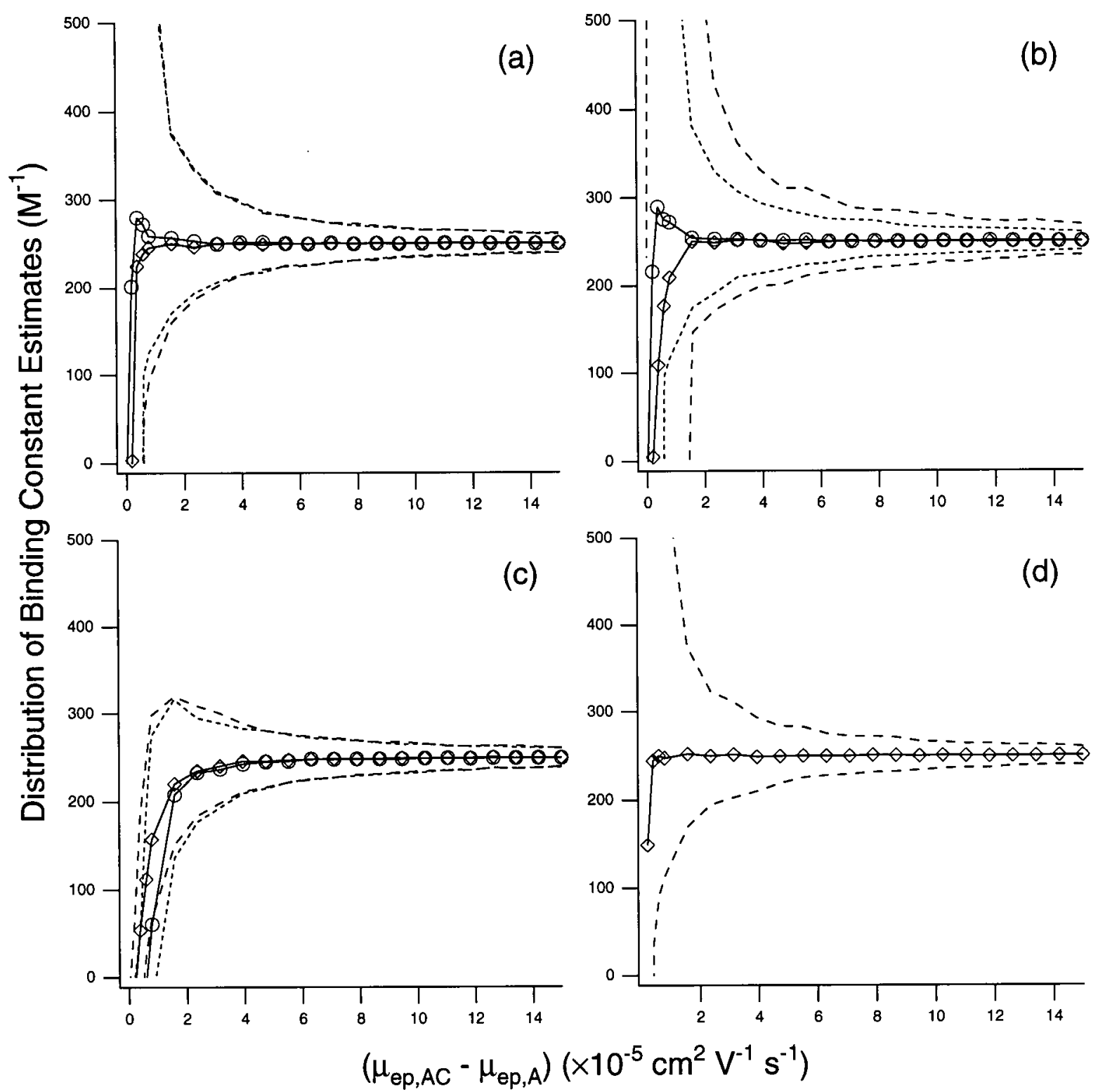

Figure 2.3.2 Distributions of the binding constants estimated using the (a) double reciprocal, (b) $y$-reciprocal, (c) $x$-reciprocal, (d) and nonlinear regression methods for $\left(\mu_{\mathrm{ap}, \mathrm{AC}}-\mu_{\mathrm{ap}, \mathrm{A}}\right)=0$ to $15 \times 10^{-5} \mathrm{~cm}^{2} \mathrm{~V}^{-1} \mathrm{~s}^{-1}$. The markers $\diamond$ and $O$ represent the medians of the unweighted and weighted methods respectively. The dashed lines define the $95 \%$ ranges for the unweighted (- - ) and weighted (-----) methods.

The $95 \%$ range of the estimated binding constants gives an indication as to the precision of the method under a certain set of conditions. A narrow range of binding constants indicates that an experiment performed under those conditions is more likely to give an estimate that is close to the actual value of the binding constant. As expected, the range of binding constant estimates generally increased as the maximum response range (i.e. $\mu_{\mathrm{ep}, \mathrm{AC}}-\mu_{\mathrm{ep}, \mathrm{A}}$ ) decreased. 
However, there were some differences in the results obtained from the different calculation methods. The $95 \%$ ranges for the $x$-reciprocal plots did not increase as drastically as with the other methods, but the results were significantly biased when the maximum response range is small. Weighting narrowed the $95 \%$ range when the $y$-reciprocal plot was used, but had little effect when the double reciprocal or $x$-reciprocal plots were used. Because the error in each data point was equal, there was no difference between the unweighted and weighted nonlinear regression methods. Figure 2.3.3 compares the magnitudes of the $95 \%$ ranges for the different calculation methods. The ranges for the nonlinear, double reciprocal, and weighted $y$-reciprocal plots were similar, indicating that the binding constant should be estimated using one of these methods. The unweighted $y$-reciprocal plots gave the widest $95 \%$ ranges. Narrow $95 \%$ ranges were achieved using $x$-reciprocal plots but bias makes it an unsuitable method for estimating the binding constant when the maximum response range is small.

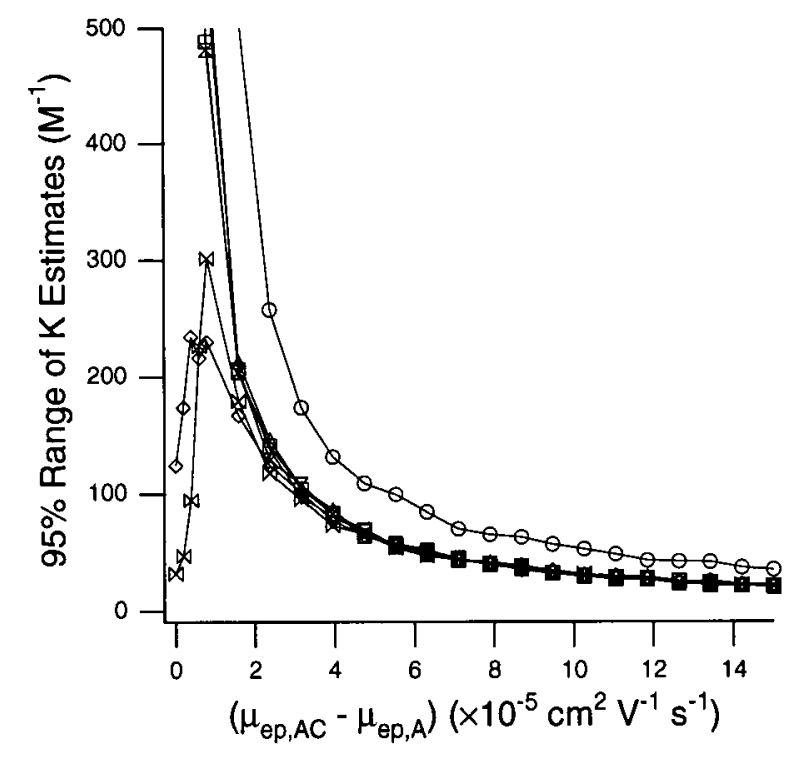

Figure 2.3.3 The $95 \%$ ranges of binding constants estimated using the double reciprocal $(\triangle)$, weighted double reciprocal $(\square), y$-reciprocal $(O)$, weighted $y$-reciprocal $(\nabla), x$-reciprocal $(\diamond)$, weighted $x$-reciprocal $(\bowtie)$, and nonlinear regression methods $(\varangle)$ for $\left(\mu_{\mathrm{ep}, \mathrm{AC}}-\mu_{\mathrm{ep}, \mathrm{A}}\right)=0$ to $15 \times 10^{-5}$ $\mathrm{cm}^{2} \mathrm{~V}^{-1} \mathrm{~s}^{-1}$. 


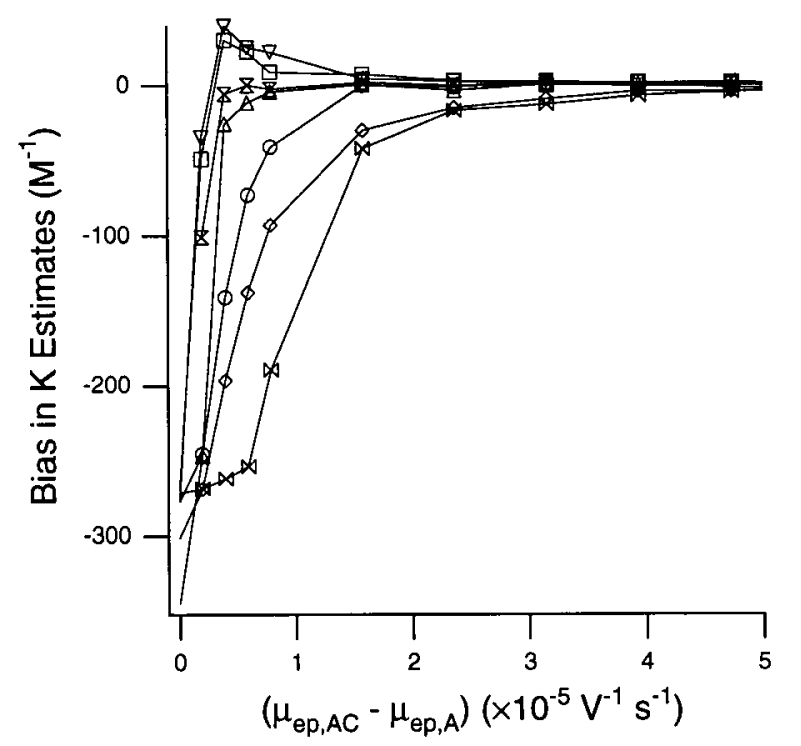

Figure 2.3.4 Bias in the distribution of binding constants estimated using the double reciprocal $(\triangle)$, weighted double reciprocal $(\square), y$ reciprocal $(O)$, weighted $y$-reciprocal $(\nabla), x$-reciprocal $(\diamond)$, weighted $x$ reciprocal $(\bowtie)$, and nonlinear regression methods $(\varangle)$ for $\left(\mu_{\mathrm{\theta p}, \mathrm{AC}}-\mu_{\mathrm{\theta p}, \mathrm{A}}\right)=0$ to $5 \times 10^{-5} \mathrm{~cm}^{2} \mathrm{~V}^{-1} \mathrm{~s}^{-1}$.

Comparing the medians of the distributions to the true value of the binding constant ( $\mathrm{K}=250 \mathrm{M}^{-1}$ in this case) demonstrates the accuracy of the different calculation methods. As shown in Figure 2.3.4, bias did become significant as the maximum response range decreased. Bias is troublesome since it cannot be eliminated through replicate measurements. Bias was most noticeable when $x$-reciprocal plots were used. Although the precision of the constants estimated using $x$-reciprocal plots was good, the accuracy was not. In this simulation, $x$-reciprocal plots consistently gave the wrong value for $\mathrm{K}$ when the maximum response range was small. Bias was also present in the double reciprocal and $y$-reciprocal plots when $\mu_{\mathrm{ep}, \mathrm{Ac}}$ approached $\mu_{\mathrm{ep}, \mathrm{A}^{*}}$. When the maximum response range was small, weighting in the double reciprocal and $y$-reciprocal plots overcompensated the error, giving rise to a slightly positive bias. Overall, bias was least significant when the nonlinear regression method was used. The combination of higher accuracy and higher precision makes the nonlinear regression the most reliable method for estimating the binding constant, especially when the maximum response range is low. This corresponds well with chapter 2.2 which demonstrated that the nonlinear 
regression method makes more reliable binding constant estimates when the range of additive concentrations is above or below the optimum range. Because of the general availability of personal computers, there does not seem to be any compelling reason to continue using the linear transformations to estimate binding constants. Although the linear transformations should not be used to estimate the binding constant, they are still useful in determining if a 1:1 equilibrium model accurately describes the data (see section 1.3.5).

\subsubsection{Semi-Empirical Prediction of the 95\% Range.}

In chapter 2.2 it was shown that the effect of the additive concentration range on the precision of the binding constant estimates (i.e. the 95\% range) could be explained by the amount of curvature in the binding isotherm. The difference in the slope of the curve at the lowest and highest additive concentration gives a measure of the amount of curvature present in the isotherm. The slope at any point on the isotherm is equal to the derivative of eq 2.1 .5 with respect to $[\mathrm{C}]$ :

$$
\frac{\partial\left(v \mu_{\mathrm{ep}}^{\mathrm{A}}\right)}{\partial[\mathrm{C}]}=\frac{\left(\mu_{\mathrm{ep}, \mathrm{AC}}-\mu_{\mathrm{ep}, \mathrm{A}}\right) \mathrm{K}_{\mathrm{AC}}}{\left(1+\mathrm{K}_{\mathrm{AC}}[\mathrm{C}]\right)^{2}}
$$

Clearly, the slope is influenced by the maximum response range as well as the binding constant and the additive concentration. As shown in Figure 2.3.1, the amount of curvature in the binding isotherm decreases as the maximum response range decreases. When the fraction of analyte bound is high, the slope of the isotherm approaches zero. When the fraction of analyte complexed is low the slope of the isotherm approaches:

$$
\frac{\partial\left(v \mu_{\mathrm{ep}}^{\mathrm{A}}\right)}{\partial[\mathrm{C}]}=\left(\mu_{\mathrm{ep}, \mathrm{AC}}-\mu_{\mathrm{ep}, \mathrm{A}}\right) \mathrm{K}_{\mathrm{AC}}
$$

Therefore, when data are collected over a large portion of the isotherm, the difference in slope 
between the lowest and highest additive concentrations is approximately:

$\Delta$ slope $\approx\left(\mu_{\mathrm{ep}, \mathrm{AC}}-\mu_{\mathrm{ep}, \mathrm{A}}\right) \mathrm{K}_{\mathrm{AC}}$

The difference in slope is almost linearly related to the maximum response range if data are collected over a substantial portion of the binding isotherm.

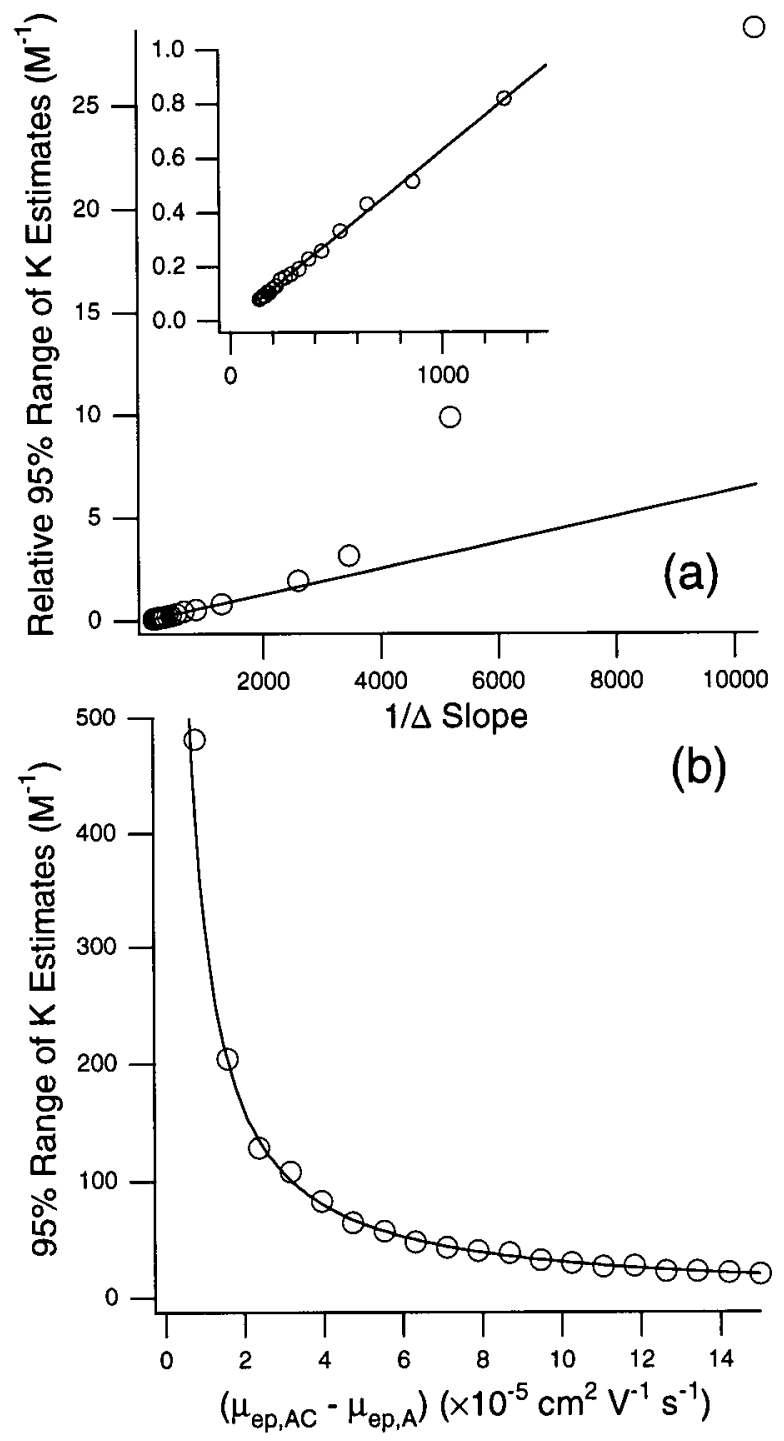

Figure 2.3.5 (a) Correlation between the relative 95\% ranges of binding constants estimated using the nonlinear regression method and the reciprocal of the difference between the slope at the lowest and highest additive concentration. (b) Comparison between the $95 \%$ ranges of the binding constants estimated using the nonlinear regression method and the curve predicted using the correlation shown in (a).

Figure 2.3.5(a) shows the correlation between the reciprocal of the difference in the slope 
and the relative $95 \%$ range of the binding constant estimates. The correlation is excellent up to a relative $95 \%$ range of one. Above one, the relative $95 \%$ ranges begin to increase faster than the reciprocal of the difference in the slope. Figure 2:3.5(b) compares the curve predicted from the correlation in Figure 2.3.5(a) to the 95\% ranges generated by the simulations. The curve corresponds well to the data, indicating that the difference between the slopes at the lowest and highest additive concentrations accurately describe the effect of the maximum response range on the precision of the binding constant estimate.

The magnitude of the maximum response range necessary to make a precise estimate of the binding constant was much higher than expected. As can be shown in Figure 2.3.5(b), for $95 \%$ of the binding constant estimates to be within $10 \%$ of the true value of the binding constant (i.e. $95 \%$ range $=50 \mathrm{M}^{-1}$ in this case), $\left(\mu_{\mathrm{ep}, \mathrm{AC}}-\mu_{\mathrm{ep}, \mathrm{A}}\right)$ must be $6.3 \times 10^{-5} \mathrm{~cm}^{2} \mathrm{~V}^{-1} \mathrm{~s}^{-1}$ or higher. This is 72 times the standard deviation of the error in the individual data points. While the size of the maximum response range required to achieve a certain level of precision will depend on other factors as well (e.g. number of data points, range of additive concentrations, data spacing, etc.), it is clear that the maximum response range must be significantly larger than the error in the individual data points.

In binding experiments, the error in the individual data points and the maximum response range are linked, similar to the way that the equilibrium constant is linked to the ligand concentration range. In order to make a reliable estimate of the binding constant, the maximum response range must be maximized, but this cannot be done at the expense of the error in the data. Therefore, experiments must be designed to both maximize the response range and minimize the error in the data. Examining the maximum response ranges listed in Table 2.3.1 gives some suggestions as to how this can be done. In UV-vis absorbance, the maximum response range can be increased by increasing the optical path length or the concentration of the substrate. In NMR studies, the maximum response range cannot be adjusted since the chemical 
shifts are intrinsic properties of the substrate and the substrate-ligand complex. However, the error in the chemical responses decreases as the field strength of the instrument is increased, allowing a more precise estimate of the binding constant. Methods for increasing the maximum response range in Michaelis-Menten kinetics and CE are less clear. Because data is collected as reaction times or migration times, perhaps emphasis should be placed on reducing the error in the time measurements.

\subsubsection{Conclusions}

It was shown that the nonlinear regression method achieved the most accurate and precise estimates for the binding constant as the maximum response range was decreased. This, combined with evidence that the nonlinear regression method is superior to the linear transformations when data is collected outside the ideal additive concentration range (see chapter 2.2), strongly suggests that the nonlinear regression method should be used when estimating binding constants.

It was also shown that the difference in the slope of the binding isotherm at the lowest and highest additive concentrations is a good indicator of the precision of a binding constant estimated using a certain set of conditions. In order to make a good estimate of the binding constant, the maximum response range must be significantly higher than the error in the individual data points. 


\subsection{References}

(1) Connors, K. A. Binding Constants - The Measurement of Molecular Complex Stability; John Wiley \& Sons: Toronto, 1987.

(2) Benesi, H.; Hildebrand, J. H. J. Am. Chem. Soc. 1949, 71, $2703-7$.

(3) Ramette, R. W. J. Chem. Educ. 1967, 44, 647-54.

(4) McBryde, W. A. E. Talanta 1974, 21, 979-1004.

(5) Slama-Schwok, A.; Teulade-Fichou, M. P.; Vigneron, J. P.; Taillandier, E.; Lehn, J. M. J. Am. Chem. Soc. 1995, 117, 6822-30.

(6) Lahiri, J.; Fate, G. D.; Ungashe, S. B.; Groves, J. T. J. Am. Chem. Soc. 1996, 118, 2347-58.

(7) Grabner, G.; Monti, S.; Marconi, G.; Mayer, B.; Klein, C.; Köhler, G. J. Phys. Chem. 1996, 100, 20068-75.

(8) Mathur, R.; Becker, E. D.; Bradley, R. B.; Li, N. C. J. Phys. Chem. 1963, 67, 2190-4.

(9) Hanna, M. W.; Ashbaugh, A. L. J. Phys. Chem. 1964, 68, 811-6.

(10) Carper, W. B.; Buess, C. M.; Hipp, G. R. J. Phys. Chem. 1970, 74, 4229-34.

(11) Seal, B. K.; Mukherjee, A. K.; Mukherjee, D. C.; Farrel, P. G.; Westwood, J. V. J. Mag. Reson. 1983, 51, 318-22.

(12) Blixt, J.; Detellier, C. J. Am. Chem. Soc. 1995, 117, 8536-40.

(13) Godínez, L. A.; Patel, S.; Criss, C. M.; Kaifer, A. E. J. Phys. Chem. 1995, 99, 17449-55.

(14) Michaelis, L.; Menten, M. L. Biochem. Z. 1913, 49, 333-69.

(15) Lineweaver, H.; Burk, D. J. Am. Chem. Soc. 1934, 56, 658-66.

(16) Eadie, G. S. J. Biol. Chem. 1942, 146, 85-93.

(17) Coolen, H. K. A.; Meeuwis, J. A. M.; van Leeuwen, P. W. N. M.; Nolte, R. J. M. J. Am. Chem. Soc. 1995, 117, 11906-13.

(18) Sun, S.; Duggleby, R. G.; Schowen, R. L. J. Am. Chem. Soc. 1995, 117, 7317-22.

(19) Corey, E. J.; Noe, M. C. J. Am. Chem. Soc. 1996, 118, 319-29.

(20) Lamm, G.; Wong, L.; Pack, G. R. J. Am . Chem. Soc. 1996, 118, 3325-31.

(21) Guedes da Silva, M. F. C.; Silva, J. A. L.; Fraústo da Silva, J. J. R.; Pombeiro, A. J. L.; Amatore, C.; Verpeaux, J.-N. J. Am. Chem. Soc. 1996, 118, 7568-73.

Aronson, J. K. Clin. Sci. 1990, 78, 247-54.

Hoang, K.-C. T. Toxicol. Lett. 1995, 79, 99-106.

Chen, T.-L.; Kennedy, M. J.; Anderson, L. W.; Kiraly, S. B.; Black, K. C.; Colvin, O. M.; Grochow, L. B. Drug Metab. Dispos. 1997, 25, 544-51.

Kwon, Y.; Morris, M. E. Pharm. Res. 1997, 14, 774-9.

Kwon, Y.; Morris, M. E. Pharm. Res. 1997, 14, 780-5.

MacIsaac, J. J.; Dugdale, R. C. Deep Sea Res. 1969, 16, $45-57$.

Mullin, M. M.; Stewart, E. F.; Fuglister, F. J. Limnol. Oceanogr. 1975, 20, 259-62.

Verity, P. G. Limnol. Oceanogr. 1991, 36, 729-50.

Wren, S. A. C.; Rowe, R. C. J. Chromatogr. 1992, 603, 235-41. 
(31) Wren, S. A. C.; Rowe, R. C. J. Chromatogr. 1992, 609, 363-7.

(32) Wren, S. A. C. J. Chromatogr. 1993, 636, 57-62.

(33) Shibukawa, A.; Lloyd, D. K.; Wainer, I. W. Chromatographia 1993, 35, 419-29.

(34) Penn, S. G.; Goodall, D. M.; Loran, J. S. J. Chromatogr. 1993, 636, 149-52.

(35) Penn, S. G.; Bergstrom, E. T.; Goodall, D. M.; Loran, J. S. Anal. Chem. 1994, 66, 2866-73.

(36) Penn, S. G.; Bergstrom, E. T.; Knights, I.; Liu, G.; Ruddick, A.; Goodall, D. M. J. Phys. Chem. 1995, 99, 3875-80.

(37) Rundlett, K. L.; Armstrong, D. W. J. Chromatogr. A 1996, 721, $173-86$.

(38) Bowser, M. T.; Sternberg, E. D.; Chen, D. D. Y. Electrophoresis 1997, 18, 82-91.

(39) Peng, X.; Bowser, M. T.; Britz-McKibbin, P.; Bebault, G. M.; Morris, J.; Chen, D. D. Y. Electrophoresis 1997, 18, 706-16.

(40) Peng, X.; Bebault, G. M.; Sacks, S. L.; Chen, D. D. Y. Can. J. Chem. 1997, 75, 507-17.

(41) Scatchard, G. Ann. N. Y. Acad. Sci. 1949, 51, 660-72.

(42) Dixon, M. Nature 1959, 184, 296-8.

(43) Dowd, J. E.; Riggs, D. S. J. Biol. Chem. 1965, 240, 863-9.

(44) Deranleau, D. A. J. Am. Chem. Soc. 1969, 91, 4044-9.

(45) Atkins, G. L.; Nimmo, I. A. Anal. Biochem. 1980, 104, 1-9.

(46) Ritchie, R. J.; Prvan, T. J. Theor. Biol. 1996, 178, 239-54.

(47) Berges, J. A.; Montagnes, D. J. S.; Hurd, C. L.; Harrison, P. L. Mar. Ecol. Prog. Ser. 1994, 114, 175-83.

(48) Sagnella, G. A. Clin. Sci. 1994, 87, 371-81.

(49) Tseng, S.; Hsu, J.-P. J. Theor. Biol. 1990, 145, 457-64.

(50) Currie, D. J. Biometrics 1982, 38, 907-19.

(51) Atkins, G. L.; Nimmo, I. A. Biochem. J. 1975, 149, 775-7.

(52) Madsen, B. W.; Robertson, J. S. J. Pharm. Pharmac. 1974, 26, 807-13.

(53) Cornish-Bowden, A.; Eisenthal, R. Biochem. J. 1974, 139, 721-30.

(54) Weber, G.; Anderson, S. R. Biochem. 1965, 4, $1942-7$.

(55) Carta, G.; Crisponi, G.; Nurchi, V. Tetrahedron 1981, 37, 2115-9.

(56) Carta, G.; Crisponi, G. J.C.S. Perkin II 1982, 53-8. 


\section{Part 3: Dynamic Complexation Capillary Electrophoresis in Nonaqueous Media}

\subsection{Analyte-Additive Interactions in Nonaqueous Solvents}

\subsubsection{Introduction}

Even though Walbroehl and Jorgenson ${ }^{(1)}$ proved that CE was not limited to aqueous solutions by demonstrating separations in acetonitrile based buffers in 1984, it is only recently, with the realization that nonaqueous solvents allow $\mathrm{CE}$ to be applied to a much wider range of analytes, that interest in solvents other than water has been renewed. Recent publications have described separations of organic ${ }^{(2,3)}$ and inorganic ions $s^{(4)}$, peptides ${ }^{(5)}$, pharmaceuticals ${ }^{(6-10)}$, and surfactants $^{(11,12)}$. Chapters 3.2 and 3.3 of this thesis discuss nonaqueous CE separations of porphyrin oligomers and monomers, respectively. Several papers have been published that describe the physical properties of nonaqueous solvents and how they can be utilized to improve separations in nonaqueous $\mathrm{CE}^{(13-15)}$.

Although the potential of nonaqueous solvents in CE has been discussed, little attention has been paid towards finding more appropriate complexation additives for nonaqueous systems. Part of the reason for the limited efforts in this area is that the additives commonly used in aqueous systems, such as surfactants and cyclodextrins, are often not as effective in nonaqueous solvents as they are in water.

This chapter reviews recent developments in nonaqueous CE, emphasizing the effect of the solvent on different types of analyte-additive interactions. Interactions driven by the solvophobic effect, electrostatic interactions (including ion-ion, ion-dipole, and dipole-dipole interactions), and donor-acceptor interactions will be discussed in detail with references to examples of CE separations. Figure 3.1.1 shows the structures of some of the complexation additives that will be discussed in this chapter. 


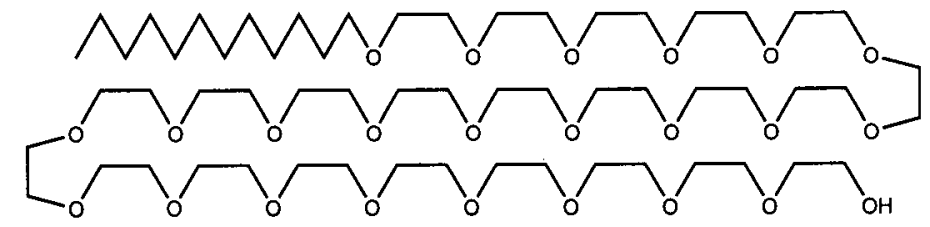

Brij 35

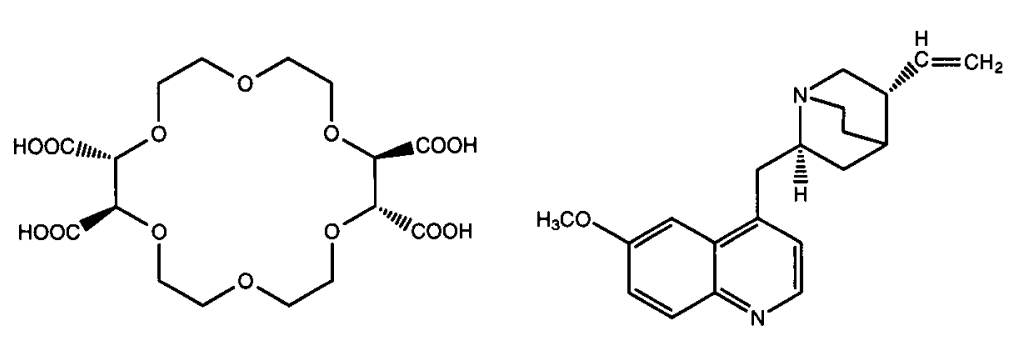

(+)-18-Crown-6 Tetracarboxylic Acid<smiles>CCCCCCCCCCCCCCCCC</smiles>

Dodecyl Trimethyl Ammonium
(-)-Quinine<smiles></smiles>

(+)-S-Camphorsulphonate<smiles></smiles>

2,4,6-Triphenylpyrylium

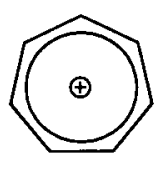

Tropylium

Figure 3.1.1 Structures of several additives used to modify analyte migration behavior in nonaqueous $\mathrm{CE}$.

\subsubsection{The Solvophobic Effect}

In order to dissolve an analyte in a solvent, a certain amount of energy is required to reorient the solvent molecules (loss of entropy), and to compensate for disrupted solvent-solvent interactions (loss of enthalpy). If the analyte is polar, analyte-solvent interactions will often offset the energy loss. Therefore, for polar analytes, a solvent is considered strong when it is also polar and can easily interact with the analyte. If the analyte does not interact with the solvent, solvation is energetically unfavorable in solvents with strong intermolecular interactions. Stronger solvents for nonpolar analytes tend to have weak solvent-solvent interactions, minimizing the energy required to disrupt the solvent. Solvent-solvent interactions are least disrupted if the analyte molecules aggregate, decreasing the analyte surface area exposed to the solvent, thus reducing the loss of both entropy and enthalpy. This causes micelles to form and 
nonpolar analytes to bind with the hydrophobic cavity of cyclodextrins in aqueous solvents.

The strength of interactions caused by the solvophobic effect is therefore related to the strength of the solvent-solvent interactions. The strengths of solvent-solvent interactions are often estimated using the polarity (dipole moment) or the dielectric constant of the solvent. However, neither the polarity nor the dielectric constant accurately reflects solvent-solvent interactions because they do not account for donor-acceptor interactions between solvent molecules, steric factors, or the density of the solvent. A more appropriate measure of solventsolvent interactions is the cohesion energy density $\left(P_{s}\right)^{(16)}$. The cohesion energy density of a solvent corresponds to the amount of energy required to disrupt all of the solvent-solvent interactions in a certain volume, and is given by:

$$
P_{S}=\frac{E_{\text {vap }}}{V_{M}}=\delta^{2}
$$

where $\mathrm{E}_{\mathrm{vap}}$ is the heat of vaporization and $\mathrm{V}_{\mathrm{M}}$ is the molar volume. The cohesion energy density is sometimes given as the solubility parameter $(\delta)$ which is equal to the square root of $P_{s}$. The cohesion energy densities of solvents commonly used in CE are listed in Table 3.1.1. Water has the highest cohesion energy density in the list, indicating solvent-solvent interactions, and therefore interactions caused by the solvophobic effect, are strongest in aqueous systems. Solvophobic interactions are generally weaker in nonaqueous solvents.

Micelles do not form in most nonaqueous solvents because hydrophobic interactions are too weak for surfactant molecules to aggregate ${ }^{(20)}$. A notable exception is formamide (FA) in which ionic surfactants, such as sodium dodecyl sulphate (SDS) and cetyl trimethyl ammonium bromide (CTAB), do form micelles. Krafft temperatures (the minimum temperature at which micelles form) are typically higher in FA than water $\left(55^{\circ} \mathrm{C}\right.$ and $43^{\circ} \mathrm{C}$ for SDS and CTAB respectively). The critical micelle concentrations $(\mathrm{cmc})$ are also higher (approximately 100 fold)(21). These properties have limited the use of micellar electrokinetic capillary 
chromatography (MECC) in FA and to our knowledge there has been no publication to date of an MECC separation using a purely nonaqueous buffer system. Reverse micelles do form in nonpolar solvents but these solvents are inappropriate for use in CE.

Table 3.1.1 Physical properties affecting analyte-additive interactions in solvents commonly used in CE.

\begin{tabular}{lcccc}
\hline Solvent & $\left.\mathrm{P}_{\mathrm{s}}(\mathrm{J} \mathrm{mL})^{-1}\right)^{(17)}$ & $\varepsilon^{(18)}$ & $\mathrm{DN}\left(\mathrm{kJ} \mathrm{mol}^{-1}\right)^{(18)}$ & $\mathrm{AN}(19)$ \\
\hline Water & 2291 & 78 & $138^{*}$ & 54.8 \\
Formamide & 1638 & 111 & $151^{\star}$ & 39.8 \\
N-Methylformamide & 992 & 182 & $205^{\star}$ & 32.1 \\
Methanol & 928 & 33 & $126^{\star}$ & 41.3 \\
Ethanol & 693 & 25 & $134^{*}$ & 37.1 \\
Acetonitrile & 655 & 38 & 59 & 18.9 \\
N,N-Dimethylformamide & 613 & 37 & 111 & 16.0 \\
Dimethylsulphoxide & 602 & 47 & 121 & 19.3 \\
N,N-Dimethylacetamide & 488 & 38 & 116 & 13.6 \\
Acetone $^{\dagger}$ & 435 & 21 & 71 & 12.5 \\
Diethyl Ether $^{\dagger}$ & 280 & 4 & 80 & 3.9 \\
\hline
\end{tabular}

"These DN have been measured indirectly and represent the donacity of the bulk solvent

${ }^{t}$ These solvents should not be used in CE due to their low flash points and high volatility. They were included in the table so a wider range of solvents could be compared.

The use of cyclodextrins in nonaqueous CE has been more successful. Both Wang and Khaledi(22), and Valkó et al.(23-25) have demonstrated chiral separations in FA, $\mathrm{N}$-methylformamide (NMF), and N,N-dimethylformamide (DMF) using $\beta$-cyclodextrin ( $\beta$-CD) as an additive. According to the cohesion energy densities listed in Table 3.1.1, interactions caused by the solvophobic effect should be the strongest, after water, in FA and NMF. Therefore, it is not surprising that solvophobic interactions have been used more successfully in these solvents. Both studies showed that interactions between the analytes and $\beta$-CD were the strongest in water, followed by FA, NMF, and finally DMF, supporting the order of cohesion energy densities listed in Table 3.1.1. Wang and Khaledi(22) measured the formation constants of the analyte-cyclodextrin complexes in water, FA, NMF, and DMF. It was shown that the 
formation constants in water were 2-4 orders of magnitude larger than those in FA. Although the values in NMF and DMF were too small to be measured accurately, the formation constants in NMF and DMF were estimated to be 3-5 and 4-6 orders of magnitude smaller than those in water, respectively. Much higher concentrations of $\beta-C D$ were necessary in the nonaqueous solvents to achieve similar separations to those in water. Obviously, changing the solvent can affect other factors (e.g. viscosity, electroosmotic flow (EOF), ionic radii, etc.) in addition to the analyte-additive interaction. If only the effect of the analyte-additive interaction is considered, a decrease in the equilibrium constants should increase the optimum additive concentration. Wang and Khaledi(22) pointed out that solvophobic interactions are so weak in the nonaqueous solvents tested that other interactions, such as dipole-dipole interactions with the hydroxyl groups around the rim of the cyclodextrin cavity, may be more important in the formation of certain analyte$\beta$-CD complexes. To support this premise, they showed that in some cases, the formation constants for those analytes that were too large to enter the cyclodextrin cavity were larger than those that could.

It appears that the interactions caused by the solvophobic effect, commonly used in aqueous $\mathrm{CE}$, will only find limited use in nonaqueous $\mathrm{CE}$. This is because the cohesion energy densities of most nonaqueous solvents are too small for significant solvophobic interactions to take place at reasonable additive concentrations. The major exceptions to this occur in FA and NMF. The literature does show cases where improved separations are achieved using these solvents ${ }^{(14,22-25)}$. In many cases the improved separation in FA and NMF is due to factors such as changes in viscosity, EOF, ionic radii, etc., instead of the analyte-additive interaction. If analyteadditive interactions are the primary separation mechanism, similar separations should be achievable in aqueous buffers at much lower additive concentrations. 


\subsubsection{Electrostatic Interactions}

Electrostatic interactions appear to be more useful in nonaqueous $\mathrm{CE}$ than solvophobic interactions. These interactions include ion-ion, ion-dipole, and dipole-dipole interactions that take place between oppositely charged ions or dipoles where there is no electron transfer between the analyte and the additive. The solvent in which these interactions take place is considered a continuous dielectric, which does not directly interact with either the analyte or the additive. The ability of the solvent to stabilize an unpaired ion or dipole within it determines the strength of the electrostatic interaction and is characterized by the dielectric constant of the solvent. The potential energy $\left(\mathrm{E}_{\mathrm{p}}\right)$ of an ion-ion interaction is given by ${ }^{(26)}$ :

$$
E_{p}=\frac{z_{A} z_{B} e^{2}}{4 \pi \varepsilon_{0} \varepsilon r}
$$

where $z_{A}$ and $z_{B}$ are the charge on ions $A$ and $B, e$ is equal to the charge of an electron, $\varepsilon_{0}$ is the permittivity of a vacuum, $\varepsilon$ is the dielectric constant (relative permittivity), and $r$ is the distance between the two ions. Similarly, the potential energy of an ion-dipole interaction is given by ${ }^{(26)}$ :

$E_{p}=\frac{z_{A} e \mu \cos \theta}{4 \pi \varepsilon_{0} \varepsilon r^{2}}$

where $\mu$ is the dipole moment and $\theta$ is the angle of the dipole.

The dielectric constant relates the strength of an electrostatic interaction in a particular solvent to that in a vacuum. Electrostatic interactions are generally strongest in solvents with low dielectric constants. Solvents stabilize unpaired ions or dipoles by reorienting their electronic, nuclear, and rotational configurations ${ }^{(27)}$. Therefore, solvents with high dipole moments tend to have higher dielectric constants than less polar solvents. Table 3.1.1 lists the dielectric constants of solvents commonly used in nonaqueous CE. Water has relatively high dielectric constant, indicating that electrostatic interactions in aqueous solvents are often weak. FA and NMF have even higher dielectric constants than water. Therefore, FA and NMF are not 
suitable solvents for electrostatic interactions. Conversely, most of the other solvents listed in Table 3.1.1 have much lower dielectric constants than water, suggesting that electrostatic interactions play a greater role in nonaqueous $\mathrm{CE}$ than in aqueous $\mathrm{CE}$.

Caution should be used when extrapolating the dielectric constants of pure solvents to $\mathrm{CE}$ buffers. The dielectric constant of a solvent is a bulk property of the pure solvent. In CE, approximating the dielectric constant of the buffer with that of the pure solvent may not always be appropriate. Much of the dielectric properties of a solvent arise from the ability of the solvent to reorient its dipole to stabilize an electric field. Solvents with lower rotational freedom tend to have smaller dielectric constants than would be expected if their polarity was the only factor considered. This explains why water and FA have lower dielectric constants than would be predicted by their dipole moments alone. Although the dipole moments of FA and NMF are similar (3.37D and 3.86D, respectively $\left.{ }^{(18)}\right)$, there are strong intermolecular interactions between FA molecules, decreasing the rotational freedom, and therefore its dielectric constant. A similar argument can be made for water. When ions are dissolved in the solvent, they create intense electric fields in their near vicinity, causing the solvent molecules to reorient towards the ion. When the solvent molecules are oriented towards the ion, their rotational freedom is restricted, creating an area with a low dielectric constant $(\varepsilon \approx 2$ for most solvents) directly surrounding the ion ${ }^{(27)}$. The overall dielectric constant for the solvent is not changed in dilute solutions but the properties of the solvent directly around the analyte and the additive may have a greater influence on the analyte-additive interaction than the bulk solvent. At higher ionic strengths, the dielectric constant of the bulk buffer may be affected as the portion of the solvent oriented towards the ions increases compared to the free solvent ${ }^{(27)}$.

Another concern is the effect of the electric field applied during the separation process. To some extent, the solvent molecules will reorient in the electric field, restricting their rotational freedom. This may cause a decrease in the observed dielectric constant of the buffer. 
Although the dielectric constant of the CE buffer may not be equal to that of the pure solvent, the values listed in Table 3.1.1 provide a qualitative description of the relative strengths of electrostatic interactions in different solvents. Unfortunately, purely electrostatic interactions are rare. Equations 3.1.2 and 3.1.3 only describe interactions in which there is no electron transfer. Most additives and analytes that undergo electrostatic interactions have electron donor or electron acceptor properties. Also, the description of electrostatic interactions presented here does not account for donor-acceptor interactions between the analyte and the solvent or the additive in the solvent. Donor-acceptor interactions will be discussed in section 3.1.4.

Because there are no donor or acceptor sites present, tetraalkyl ammonium ions are a class of additives that predominantly interact with anionic analytes through electrostatic interactions. Kranack ${ }^{(28)}$ has recently shown the applicability of dodecyltrimethylammonium ions in modifying the electrophoretic mobilities of nitrophenols in a methanol based buffer. The ammonium-phenolate interactions are examples of an electrostatic ion-ion interaction. It was also demonstrated that the ammonium-nitrophenolate interactions were much stronger in a methanol based buffer (approximately $175-450 \mathrm{M}^{-1}$ ) than in aqueous buffers (too low to measure accurately). This was expected since the dielectric constant of methanol is much lower than that of water. Walbroehl and Jorgenson ${ }^{(29)}$ demonstrated the usefulness of tetrahexyl ammonium as an additive in the separation of a group of polycyclic aromatic hydrocarbons (PAHs) in an acetonitrile based buffer. The ammonium ions induce a dipole on the PAH, allowing an electrostatic interaction to take place. Chapters 3.2 and 3.3 describe the separation of porphyrin acid oligomers and monomers based on a purely electrostatic ion-dipole interaction with a polyether surfactant.

\subsubsection{Donor-Acceptor Interactions}

In many cases, the purely electrostatic description of ion-ion, ion-dipole, and dipoledipole interactions is an over simplification. Although most researchers in CE have attempted to 
rationalize the effect of the solvent on analyte-additive interactions using only the dielectric constant of the solvent, many physical chemists have realized that the dielectric constant does not sufficiently describe many solvent dependent effects ${ }^{(16,27)}$. The discrepancies largely arise from the assumption in the electrostatic model that there is no electron transfer between the analyte and the additive. It is also assumed that there is no electron transfer between the solvent and the analyte or additive. As pointed out in the previous section, this is rare in most CE systems.

More often, ion-ion, ion-dipole, and dipole-dipole complexes will undergo donoracceptor interactions as well as electrostatic interactions. Molecules with free electron pairs, often present on oxygen or sulfur atoms, are electron donors. There are two types of electron acceptors: Lewis acids, which typically have unfilled orbitals (e.g. metal cations), and hydrogen bond donors (e.g. hydroxyls) in which the donated hydrogen is an electron acceptor (e.g. water, methanol). An interaction between an analyte and an additive that has both electrostatic and donor-acceptor properties can be described using the following equilibrium:

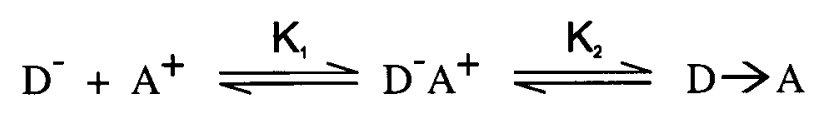

where $\mathrm{D}$ is an electron donor and $\mathrm{A}$ is an electron acceptor. $\mathrm{D}^{-}$and $\mathrm{A}^{+}$first form a complex without electron transfer, then, if conditions allow, complete the electron transfer process. In the present context either A or D could be the analyte. Although D and A are initially shown with charges in eq 3.1.4, the equilibrium is equally representative of ion-dipole and dipole-dipole interactions. The equilibrium constant $\mathrm{K}_{1}$ describes the formation of an ion pair through a purely electrostatic interaction as described in the previous section. $\mathrm{K}_{2}$ shows that further interaction can take place with electron transfer between $\mathrm{A}$ and $\mathrm{D}$. While the division between the electrostatic and donor-acceptor processes are not as distinct as shown in eq 3.1.4, this approach is useful in showing that both processes need to be considered to understand the binding process. If very 
little electron transfer occurs (i.e. $\mathrm{K}_{1} \gg \mathrm{K}_{2}$ ), the interactions can be accurately described by only considering the electrostatic interactions. When electron transfer does take place, $\mathrm{K}_{1}$ is often smaller than $\mathrm{K}_{2}$, and the donor-acceptor interaction dominates ${ }^{(27)}$. Researchers have shown that a large number of solvent dependent effects can be described when both the electrostatic and donor-acceptor interactions are considered ${ }^{(19,27,30)}$.

The previous section has shown that the dielectric constant is useful in describing the effect of the solvent on electrostatic interactions. Donor-acceptor interactions are affected by the donor and acceptor properties of the solvent. When the solvent enters the competitive equilibrium for the binding sites on the analyte, the interaction can be written as:

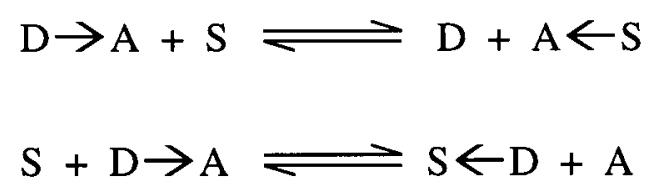

where $S$ is a solvent with donor properties (in eq 3.1.5) or acceptor properties (in eq 3.1.6). Often the solvent can act as both an electron donor and an electron acceptor so the equilibria shown in eqs 3.1.5 and 3.1.6 act in concert. Clearly, the donor and acceptor properties of the solvent have an effect on the interactions between the analyte and the additive. As the donor and acceptor strengths of the solvent increase, the formation constant of an analyte-additive complex formed through donor-acceptor interactions will decrease.

Gutmann $^{(30)}$ developed an empirical measure of the donor and the acceptor strengths of solvents. The donacity of a solvent is given by its donor number (DN). The DN of a solvent is traditionally determined by measuring $-\Delta \mathrm{H}$ for the formation of solvent- $\mathrm{SbCl}_{5}$ complexes in dilute dichloroethane solutions ${ }^{(30)}$. Therefore, a high DN indicates the solvent is a strong electron donor. Other methods for measuring donacity were later developed that correlated well with Gutmann's donacity scale(18,27,31). For solvents with strong intermolecular interactions (e.g. water), however, the donacities of their dilute solutions in another solvent and the donacities 
when they are used as bulk solvents can differ significantly ${ }^{(18,31)}$. The newer methods measure the donacity of bulk solvents while Gutmann's method measures DN for dilute solutions of the solvent. Gutmann realized that his method did not account for intermolecular interactions and even suggested that the DN measured for water represented the donacity of gaseous water ${ }^{(30)}$. The DN for the bulk solvents are listed in Table 3.1.1 for solvents where there are discrepancies between dilute solutions and the bulk solvent.

Acceptor numbers (AN) of solvents are found by comparing the ${ }^{31} \mathrm{P}$ NMR shifts of triethylphosphine oxide $\left(\mathrm{Et}_{3} \mathrm{PO}\right)$ when bound to the solvent molecules ${ }^{(19,27,30)}$. The values are normalized to give a dimensionless quantity with the $\mathrm{AN}$ for $\mathrm{SbCl}_{5}$ arbitrarily set at 100 . Higher AN indicate higher electron acceptor properties for the solvent. It should be noted that AN only relate the relative acceptor properties of the solvents and cannot be directly correlated to the DN scale. The AN for various nonaqueous solvents are listed in Table 3.1.1. Solvents that can act as hydrogen bond donors (e.g. water, methanol, and FA) generally have higher AN than those that cannot (e.g. DMSO, acetonitrile, and DMF).

The specific analyte-additive interaction must be considered to determine whether the donor or acceptor properties of the solvent will have the most influence. Generally speaking, donor-acceptor interactions will be the weakest in water, FA, NMF because these solvents are all relatively strong donors and acceptors themselves.

Most of the analyte-additive interactions used to modify analyte migration in nonaqueous $\mathrm{CE}$ involve ion-ion, ion-dipole, or dipole-dipole interactions with some donor-acceptor properties. An example of this is the chiral separation of a group of amino drugs demonstrated by Bjørnsdottir et al. ${ }^{(9)}$. They used (+)-S-camphorsulphonate as an additive and acetonitrile as the solvent. It was pointed out that a two-point interaction was necessary between the analyte and the additive in order to obtain chiral resolution. The sulphonate group and the ketone on the additive acted as electron donors. The amines on the analytes acted as electron acceptors. 
Donor-acceptor interactions are strong in acetonitrile because of its low DN and AN. If the dielectric constant was the only factor considered, this result would have been difficult to explain. The dielectric constant for acetonitrile is higher than that of methanol because of the strong dipole present in acetonitrile. The higher dielectric constant suggests that if the analyteadditive interaction was electrostatic, binding should be weaker in acetonitrile than that in methanol. The observations support the premise that the interactions take place through donoracceptor interactions, which are much stronger in acetonitrile than methanol because of its weaker donor and acceptor properties.

Polyethers are promising as electron donating additives. Mori et al. ${ }^{(32)}$ have recently described the separation of primary amino enantiomers using a chiral crown ether $((+)-18$-crown6 tetracarboxylic acid). Although the separation was carried out in FA, which typically gives weak donor-acceptor interactions, good chiral separation was achieved. Polyethers tend to give stronger than expected binding due to the multiple binding sites available. It was also noted that no separation was achieved in aqueous $\mathrm{CE}$ using the same crown ether, illustrating that the combination of high DN and high AN makes donor-acceptor interactions in water even weaker than in FA. Other crown ethers, short chain polyethers, and polyether surfactants should prove useful as additives in nonaqueous CE due to the number of polyethers available, their low cost, and their low UV absorbance.

Small organic and inorganic ions are the simplest additives for use in nonaqueous CE.

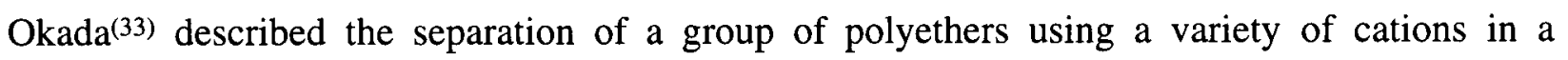
methanol buffer. Binding constants were measured between the polyethers and potassium, sodium, ammonium, methyl ammonium, dimethyl ammonium, ethyl ammonium, and propyl ammonium ions. The cations acted as electron acceptors. In aqueous CE, donor-acceptor interactions with the ions listed above are typically too weak to be useful in modifying analyte mobility. In methanol, with its lower dielectric constant, DN, and AN, the interactions are 
stronger. In the separation of alkali and alkaline earth metals in a methanol buffer, SalimiMoosavi and Cassidy ${ }^{(34)}$ suggested that interactions with the acetate ions in the buffer affected the analyte mobilities. This was confirmed by a decrease in analyte mobilities when acetonitrile was added to the buffer. The decrease in mobility was attributed to an increase in the interaction between the analytes and the acetate ions. Again this can not be explained by the change in the dielectric constant of the solvent since acetonitrile and methanol have similar dielectric constants. The lower DN and AN of acetonitrile promote increased donor-acceptor interactions between the metal ions and the acetate ions.

Miller et al.(35) used "charge-transfer" interactions to develop a separation of polycyclic aromatic hydrocarbons (PAHs) in a acetonitrile buffer. The tropylium ion and the 2,4,6triphenylpyrylium ion were used as charge-transfer additives. Contrary to what was initially believed to be a charge-transfer complexation, the authors showed that the dominant effect is from induced dipole interactions. Because this technique uses a planar cation, it is more effective for PAH molecules which take part in cation- $\pi$ electron interactions. These cations should be used with caution, however, because their reactivity limits their applicability to certain analytes and solvents. Another concern is the high UV absorbance of these additives.

Stalcup and $\mathrm{Gahm}^{(36)}$ took advantage of a two point interaction with quinine to achieve the enantiomeric separation of several N-3,5-dinitrobenzoylated amino acids in a methanolic buffer. The protonated amine on quinine acted as an electron acceptor while the quinoline was able to undergo $\pi-\pi$ interactions with aromatic groups on the derivatized amino acids.

\subsubsection{The Complexity of Analyte-Additive Interactions in Nonaqueous CE}

Analyte-additive interactions are generally more complex in nonaqueous $\mathrm{CE}$ than in aqueous systems. Most additives in aqueous CE interact with the analyte through hydrophobic 
effects. Electrostatic interactions and donor-acceptor interactions are generally weak, and with proper choice of conditions their effects can be minimized. Therefore it is possible to focus on the effect of a particular interaction (usually a hydrophobic interaction) in aqueous CE.

In nonaqueous $\mathrm{CE}$, electrostatic and donor-acceptor interactions dominate. As shown in the previous section, a wide range of molecules and ions undergo these types of interactions. Many ions or other buffer additives can be expected to undergo such types of interaction with the analyte. For example, a typical organic anion can be expected to interact the buffer cations (e.g. sodium or ammonium ions), be involved in acid-base equilibrium, as well as to interact with any additive present in the buffer. The increase in the strength of the interactions between the analyte and the constituents of the buffer may contribute to the improved separation observed in many nonaqueous systems. This improvement is usually attributed to the autoprotolytic properties of the solvent or differences in the solvation radii of the analytes ${ }^{(7,14,15)}$. The general separation theory developed in chapter 1.2 should be useful in understanding the complicated equilibria that are common in nonaqueous CE. As shown in chapters 1.3 and 1.4 , the theory can easily be adapted to cases where multiple-additive or multiple-stoichiometry interactions take place.

\subsubsection{Conclusions}

Clearly the solvent plays an important role in determining the effectiveness of certain additives in $\mathrm{CE}$. Interactions driven by the solvophobic effect are generally much weaker in nonaqueous solvents than in water. As a result, those additives that primarily bind through solvophobic interactions will only be useful in solvents with high cohesion energy densities such as FA. Ion-ion, ion-dipole, and dipole-dipole interactions are stronger in most nonaqueous solvents than in water. Both electrostatic and donor-acceptor properties need to be considered to understand the effect of the solvent on these interactions. Since all CE buffers contain ions and 
molecules capable of undergoing electrostatic and/or donor acceptor interactions, analyteadditive interactions in nonaqueous $\mathrm{CE}$ are generally more complicated than those in aqueous systems. 


\subsection{Development and Application of a Nonaqueous Capillary Electrophoresis System for the Analysis of Porphyrins and Their Oligomers (PHOTOFRIN ${ }^{\circledR}$ )}

\subsubsection{Introduction}

PHOTOFRIN ${ }^{\circledR}$ is a complex porphyrin mixture isolated from hematoporphyrin derivative (HPD) used in the photodynamic therapy of tumors ${ }^{(37-39)}$. Photodynamic therapy is a treatment in which a photosensitizing agent is first localized in a tumor and then irradiated with a relatively low intensity light source. Singlet oxygen generated during the degradation process destroys the targeted cells ${ }^{(39)}$. Lipson et al. first described the preparation of hematoporphyrin derivative as well as its clinical potential in $1961^{(40)}$. The first step in the preparation of hematoporphyrin derivative is the reaction of hematoporphyrin (HP) dihydrochloride with acetic acid/sulfuric acid followed by treatment with dilute base. The product of this step includes the acetates of hematoporphyrin and its dehydration products: hydroxyethylvinyldeuteroporphyrin (HVD) and protoporphyrin (PP). This mixture is then hydrolyzed in a $0.1 \mathrm{M}$ sodium hydroxide solution to give HPD. This mixture contains approximately $20 \%$ HP, 20-30\% HVD and 3-5\% $\mathrm{PP}^{(38)}$. The remaining 50\% consists of oligomers containing up to fifteen porphyrin base units connected through ester, ether and carbon-carbon linkages ${ }^{(41)}$. Studies have shown that the most pharmaceutically active compounds are contained in the oligomeric fraction ${ }^{(37-39)}$. PHOTOFRIN ${ }^{\circledR}$ is an enriched form of HPD in which the oligomeric component makes up 80$90 \%$ of the mixture( ${ }^{(38)}$. The oligomerization reaction and the possible products are shown in Figure 3.2.1. The products can be ether linked, ester linked, or any combination of the two.

Characterization of the oligomeric component of PHOTOFRIN ${ }^{\circledR}$ has been hampered by the large number of compounds produced by oligomerization at the various sites indicated in Figure 3.2.1. Most attempts at overcoming this problem have focused on developing liquid chromatography methods ${ }^{(42-45)}$. It has often been necessary to use multiple columns or simplify the sample through decomposition. Although this gives some insight into the properties of the 
compounds in the oligomeric fraction, much information is lost using these strategies.

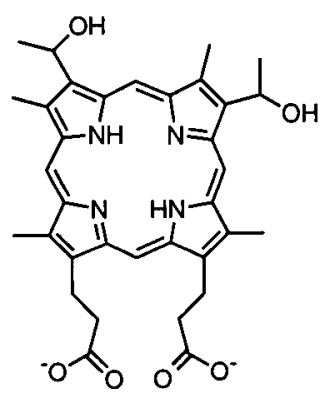

HP

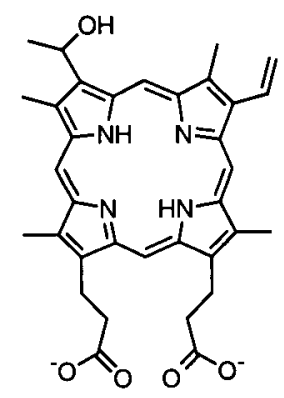

HVD

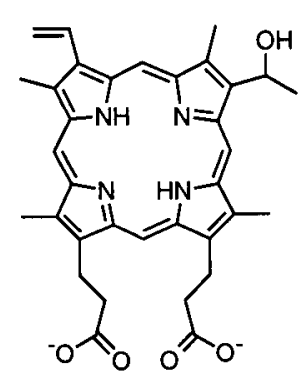

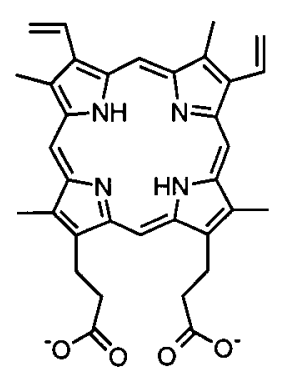

PP

1) $\mathrm{H}_{2} \mathrm{SO}_{4} / \mathrm{ACOH}$

2) $0.1 \mathrm{M} \mathrm{NaOH}$

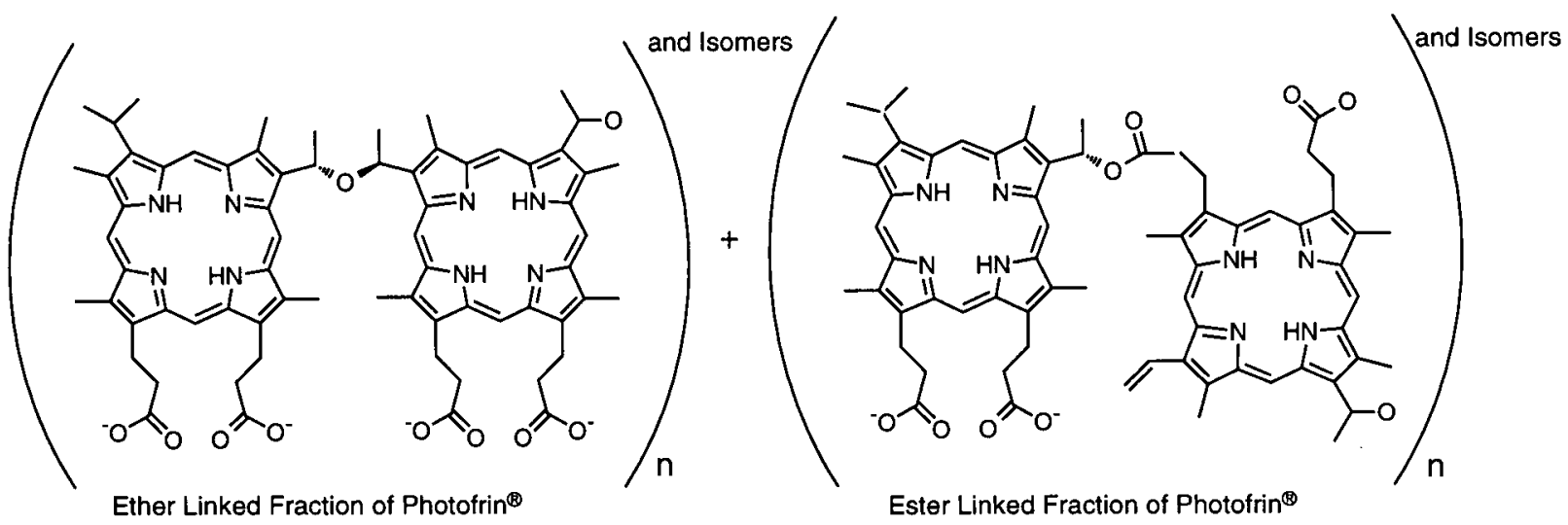

Figure 3.2.1 The oligomerization reaction of the porphyrin monomers. Both the monomers and the oligomers are present in PHOTOFRIN ${ }^{\circledR}$.

Micellar electrokinetic capillary chromatography (MECC) separations have been previously described for monomeric porphyrins ${ }^{(46-50)}$ but attempts at developing a method for the analysis of the oligomeric fraction in PHOTOFRIN ${ }^{\circledR}$ have been largely unsuccessful. Many of the problems in developing a CE method for the oligomeric component seem to arise from the aggregation of porphyrins in aqueous buffers. Interactions between different sample components, which decrease peak efficiency, are more significant in aqueous solutions. In this study the problem is overcome through the use of a nonaqueous buffer system.

Since micelles will not form in most nonaqueous buffer systems ${ }^{(20)}$, a more suitable complexation additive is needed to manipulate the selectivity of the separation. As discussed in 
chapter 3.1, electrostatic and electron-transfer interactions are stronger in most nonaqueous solvents. Some of these interactions are too weak in aqueous systems to effect the selectivity of a separation but become significant in nonaqueous systems. In this chapter, the dipolar nature of a polyether compound is used to modify the separation of PHOTOFRIN ${ }^{\circledR}$.

\subsubsection{Experimental}

Separations were performed on a Beckman P/ACE 5000 automated capillary electrophoresis system (Beckman Instruments Inc., Mississauga, Ont., Canada). Uncoated capillaries (Polymicro Technologies, Phoenix, AZ) were used with inner diameters of $75 \mu \mathrm{m}$, outer diameters of $375 \mu \mathrm{m}$ and lengths of $57 \mathrm{~cm}$. New capillaries were rinsed for 20 minutes with $1 \mathrm{M}$ aqueous $\mathrm{NaOH}$ (BDH Inc., Toronto, Ont., Canada) followed by nonaqueous separation buffer for 20 minutes. The capillary was then allowed to equilibrate overnight before being used. A 1 minute rinse of $0.2 \mathrm{M}$ sodium hydroxide in methanol followed by a 2 minute rinse of buffer was performed before each separation. The sample was introduced using a 1 second low pressure injection. Separations took place at a potential of $-30 \mathrm{kV}$ and a temperature of $20^{\circ} \mathrm{C}$. Absorbance detection was performed using a Beckman UV-Vis detector at $400 \mathrm{~nm}$. Fluorescence was induced with the $488 \mathrm{~nm}$ line of a $4 \mathrm{~mW}$ argon ion laser (Beckman). Emission was monitored at $620 \mathrm{~nm}$ with a Beckman fluorescence detector. Data were collected and processed using System Gold software (Beckman) and a 386 PC computer.

Buffers were prepared in HPLC grade methanol (Fisher Scientific, Nepean, Ont., Canada) by adding the appropriate amount of 3-[cyclohexylamino]-1-propanesulfonic acid (CAPS) (Sigma Chemical Co., St. Louis, MO, USA) and brij 35 (Aldrich Chemical Co., Inc., Milwaukee, Wis., USA). Unless otherwise noted the buffer concentration was $20 \mathrm{mM}$ CAPS $(\mathrm{pH}=10.5) / 5 \mathrm{mM}$ brij 35 . The $\mathrm{pH}$ of the buffer was adjusted with $0.2 \mathrm{M}$ sodium hydroxide in methanol before the solution was made up to its final volume. The $\mathrm{pH}$ of the final solution was 
then confirmed. It should be noted that this is not a measurement of $\mathrm{pH}$ in the conventional sense, but is used to ensure the buffer is prepared reproducibly. PHOTOFRIN ${ }^{\circledR}$, hematoporphyrin, hydroxyethylvinyldeuteroporphyrin and protoporphyrin were provided by QLT PhotoTherapeutics Inc. (Vancouver, BC, Canada). Solutions of PHOTOFRIN ${ }^{\circledR}$ and the porphyrin standards were made up weekly in $2 \mathrm{mM}$ CAPS $(\mathrm{pH}=10.5) / 0.5 \mathrm{mM}$ Brij 35 in methanol. Unless otherwise noted PHOTOFRIN ${ }^{\circledR}$ solutions were prepared at a concentration of $500 \mathrm{ppm}$.

\subsubsection{Effect of the EOF on Resolution}

The general expression for resolution is given in eq 1.2.22:

$$
\mathrm{R}_{\mathrm{S}}=\frac{\mathrm{t}_{\mathrm{R}}^{\mathrm{B}}-\mathrm{t}_{\mathrm{R}}^{\mathrm{A}}}{\frac{1}{2}\left(\mathrm{~W}_{\mathrm{A}}+\mathrm{W}_{\mathrm{B}}\right)}=\frac{\sqrt{\mathrm{N}}}{4}(\gamma-1)
$$

where the separation factor $\gamma$ (written in terms of mobility) is:

$$
\gamma=\frac{\mu^{\mathrm{A}}}{\mu^{\mathrm{B}}}=\frac{\mu_{\mathrm{ep}}^{\mathrm{A}}+\mu_{\mathrm{eo}}}{\mu_{\mathrm{ep}}^{\mathrm{B}}+\mu_{\mathrm{eo}}}
$$

If the electrophoretic mobility of the analyte and the electroosmotic mobility are in the same direction, any increase in $\mu_{\mathrm{eo}}$ decreases the resolution. The EOF pushes the sample off the capillary before the full effect of the potential can be experienced by the analytes. In this case the EOF should be reduced as much as possible to improve resolution.

If the electrophoretic mobility of the analyte and the EOF are in opposite directions the resolution approaches infinity as the magnitude of $\mu_{\mathrm{eo}}$ approaches $\mu_{\mathrm{ep}}^{\mathrm{B}}$. Resolution improves as the length of time that the analytes are held in the electric field increases. Unfortunately, analysis time approaches infinity as resolution approaches infinity. Lucy et al. have achieved the 
separation of isotopic chloride ions based on the same principle ${ }^{(51)}$. Jorgenson $e t$ al. achieved a similar result using a counterbalancing hydrodynamic flow to keep the analyte in the electric field for longer times ${ }^{(52)}$.

In most aqueous systems the electroosmotic mobility is much larger in magnitude than the electrophoretic mobility and forces the analytes to move in the same direction as $\mu_{\mathrm{eo}}$. Thus, the EOF should be reduced to increase $\gamma$, thereby improving the resolution. However, when the magnitude of the EOF is less than the electrophoretic mobilities of the analytes, as shown in this experiment, the EOF should be increased to counterbalance $\mu_{\mathrm{ep}}^{\mathrm{B}}$, and increase $\gamma$.

Since the electroosmotic mobilities of methanol based buffers are relatively small, the potential of closely matching $\mu_{\mathrm{eo}}$ and $\mu_{\mathrm{ep}}^{\mathrm{B}}$ exists. This advantage is often overlooked when considering nonaqueous buffers and to date has not been investigated fully. In the case of the porphyrins in PHOTOFRIN ${ }^{\circledR}$, the mobilities of the analytes are greater in magnitude than the EOF. For this reason a separation potential of $-30 \mathrm{kV}$ is necessary. This means that analytes with the smallest electrophoretic mobilities will come off the capillary last. Analytes with the smallest electrophoretic mobilities will also match the electroosmotic mobility the best, giving the highest resolution. This is important in the analysis of PHOTOFRIN ${ }^{\circledR}$ since the oligomers have the lowest mobility. In aqueous systems, where the EOF is the driving force, the oligomers would reach the detector first and have the lowest resolution. In the nonaqueous system the electrophoretic mobility is the driving force, causing the oligomers to come off the capillary last, resulting in a higher resolution. This is a better scenario since the oligomer region is the most complicated part of the electropherogram. It should also be noted that this is the only case in which an increase in EOF can be used to improve the resolution. 


\subsubsection{Effect of Brij 35}

Brij 35 is a common nonionic polyether surfactant. It was used in this experiment to control the selectivity of the separation as well as help reduce aggregation of the oligomeric portion of PHOTOFRIN ${ }^{\circledR}$. Some separation was achieved without the use of buffer additives, but the efficiency was approximately 8,000 plates and the analysis time was over 90 minutes. Peak broadening can result if intercomponent interactions become significant. The addition of brij 35 increased the efficiency of the separation to approximately 200,000 theoretical plates. This increase in efficiency can be attributed to brij 35 disrupting the aggregation of PHOTOFRIN ${ }^{\circledR}$.

Brij 35 was chosen as a buffer additive because of its potential for dipolar interactions as well as its high solubility in methanol. The structure of brij 35 is shown in Figure 3.1.1. Other potential additives such as sodium dodecyl sulfate (SDS), cetyltrimethyl ammonium bromide (CTAB) and Triton QS-15 were tested, but were not as successful as brij 35. Although brij 35 is not expected to form micelles in methanol, it should undergo complexation with the analyte through dipolar interactions. Dipole bonds between the additive and analyte become stronger in less polar solvents and have a greater effect on the separation than in aqueous systems. With the addition of brij 35 , separation selectivity becomes dependent on the analyte-additive interactions as well as differences in mobility. Figure 3.2.2 illustrates the effect brij 35 has on the separation. 


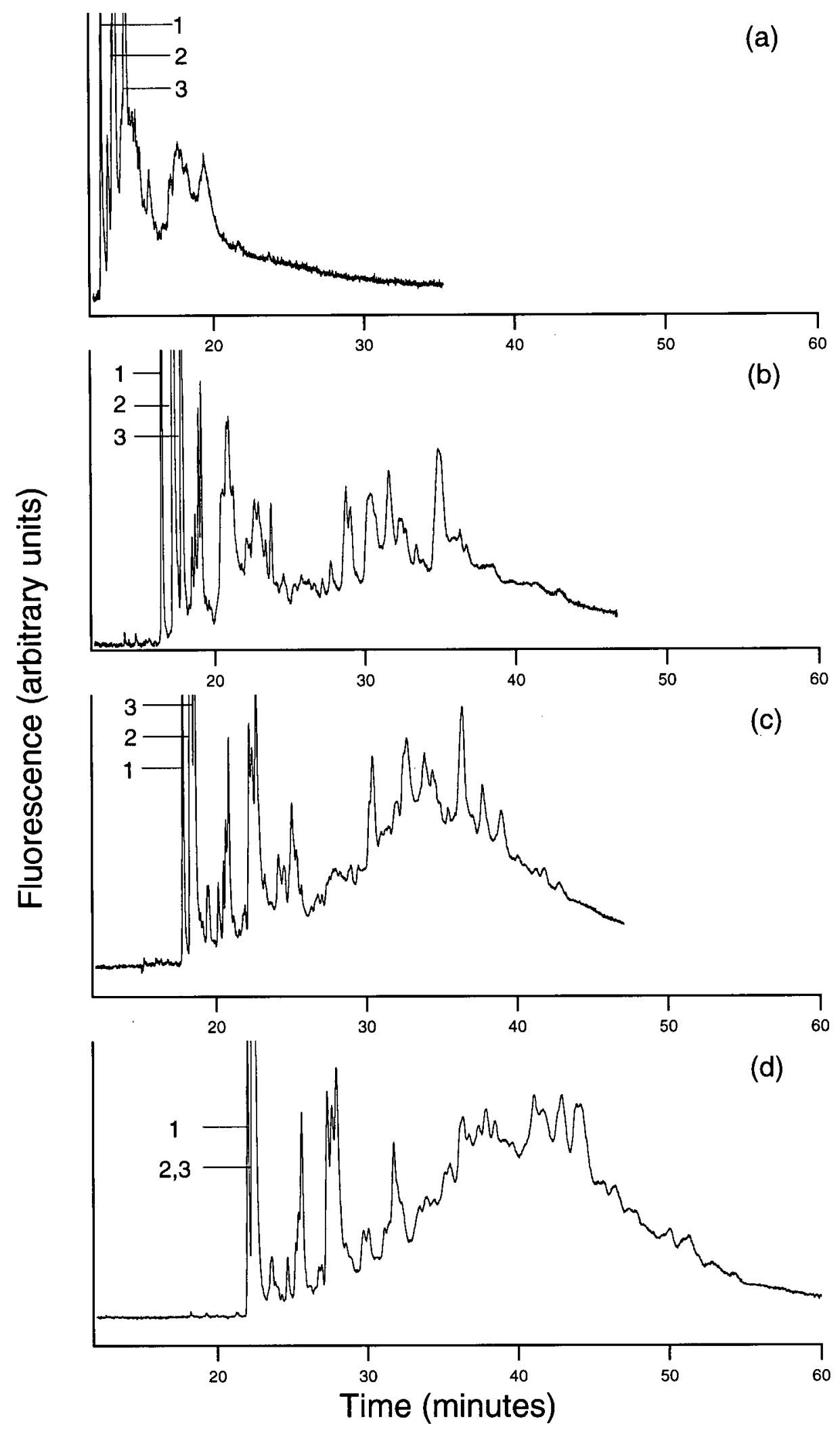

Figure 3.2.2 Effect of varying brij 35 concentration on the nonaqueous CE separation of PHOTOFRIN ${ }^{\circledR}$. The concentrations of brij 35 are (a) 2 $\mathrm{mM}$, (b) $5 \mathrm{mM}$, (c) $10 \mathrm{mM}$ and (d) $20 \mathrm{mM}$. The numbered peaks are: (1) PP , (2) HVD, and (3) HP. 
The three monomeric porphyrins migrate off the capillary first, followed by the oligomeric fraction. The elution order of the monomers supports the premise that complexation takes place through dipolar interactions. The main mechanism of separation should be differences in complexation since all three porphyrins are similar in size and charge. Complexation with brij 35 will slow the migration of the analyte. Therefore, the monomer with the most hydrogen bonding sites should be the last to reach the detector. This statement is in agreement with the elution order of PP followed by HVD and HP. At lower brij 35 concentrations, the separation is mainly caused by the differences in the extent of complexation. At higher brij 35 concentrations the monomer peaks begin to converge, because most of the analyte has complexed with the additive. The separation is now governed by the difference in the charge to size ratio of the monomer-additive complex, not the extent of complexation. The charge to size ratio of the complexes will be similar.

The addition of brij 35 had a more dramatic effect on the oligomeric component of PHOTOFRIN ${ }^{\circledR}$. At low concentrations there is almost no separation in this region due to poor efficiency. Efficiency in the oligomeric fraction improved with increased concentrations of brij 35. At higher additive concentrations complexation increases, the selectivity of the separation improves and the peaks spread over a longer time. Because of the complexity of the sample, one set of conditions may not be satisfactory to study all the components of PHOTOFRIN ${ }^{\circledR}$. If separation of the monomers is not necessary, the concentration of brij 35 can be increased to improve resolution in the oligomeric fraction by further breaking up the oligomer aggregates. A concentration of $5 \mathrm{mM}$ was chosen for this study as a compromise between separation in the monomer and the oligomer fractions. 


\subsubsection{Effect of Solvent Polarity}

One of the major advantages of nonaqueous buffers is the ease with which their properties can be adjusted. An example of this can be illustrated in the present experiment by modifying the solvent's polarity through the addition of water. Figure 3.2.3 shows the effect on the separation when the buffer is made up in varying concentrations of water.

There are several factors to consider in understanding the effect that the addition of water has on the separation. The first is the aggregation of the sample in more polar solvents. As has been stated earlier, the oligomeric, and to a lesser extent monomeric, fraction of PHOTOFRIN ${ }^{\circledR}$ aggregates in water. For this reason the addition of water to the buffer should increase the interactions between sample components, decreasing the efficiency of the separation. Figure 3.2.4 shows this trend in a plot of monomer peak efficiency $v s$. water content. Examination of the electropherograms in Figure 3.2.3 shows that the trend holds for the oligomeric fraction as well. Caution should be taken in interpreting these results since efficiency is not independent of time in CE. Theory predicts that plate counts should increase with migration time ${ }^{(51)}$ but this does not account for band broadening caused by intercomponent interactions. These interactions may decrease peak efficiency as migration time increases, and may be a contributing factor in the trend illustrated in Figure 3.2.4.

Another consideration is the effect on the EOF. The dielectric constant of the buffer will increase with the addition of water, thereby increasing the EOF. As proposed earlier, an increase in the EOF will improve separation by increasing $\gamma$. This is demonstrated in Figure 3.2.3 where the peaks become spread over a longer time as the fraction of water in the buffer is increased. Resolution improves with increasing water concentration up to approximately $10 \%(\mathrm{v} / \mathrm{v})$. Above this concentration, band broadening caused by intercomponent interactions becomes more significant and resolution decreases. When the water content is raised to $50 \%$ the electroosmotic mobility becomes strong enough to pull the sample to the negative electrode and the separation 
degrades quickly.

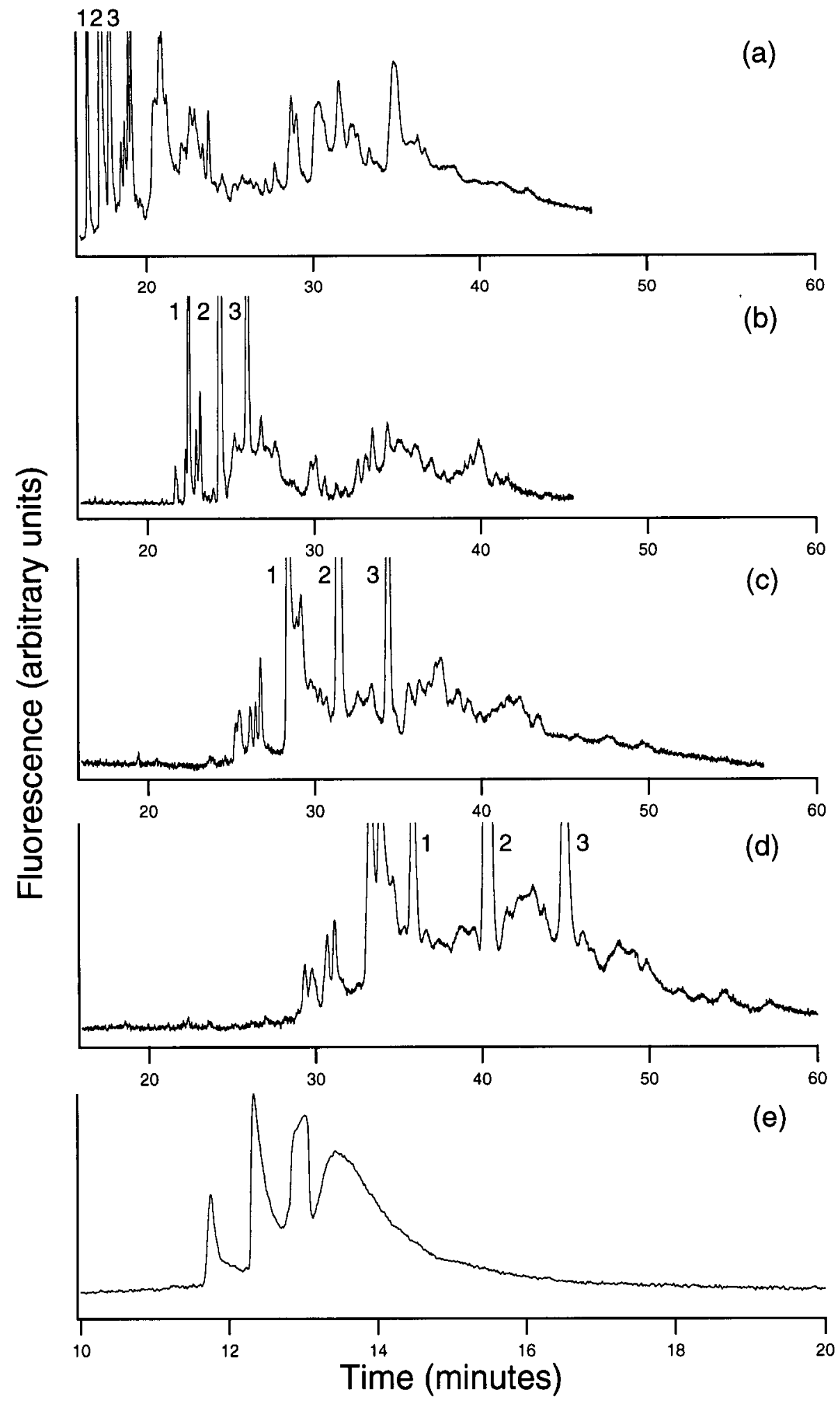

Figure 3.2.3 Effect of varying water content on the separation of PHOTOFRIN ${ }^{\circledR}$. The water contents $(\% \mathrm{v} / \mathrm{v})$ of the buffers are (a) $0 \%$, (b) $6.7 \%$, (c) $13.3 \%$, (d) $20 \%$, (e) $50 \%$. It should be noted that the separation potential is $-30 \mathrm{kV}$ for (a) - (d), and $+30 \mathrm{kV}$ for (e). The numbered peaks are: (1) PP, (2) HVD, and (3) HP. 


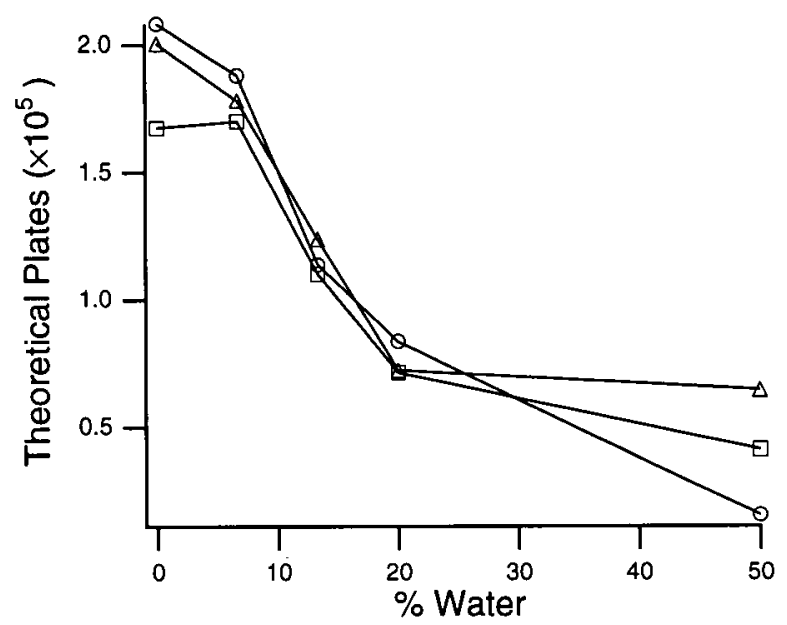

Figure 3.2.4 Plot of separation efficiency (theoretical plate number) vs. buffer water content $(\% \mathrm{v} / \mathrm{v})$ for the porphyrin monomers.

The last factor to consider pertains to the complexation between the analyte and brij 35 . Assuming that dipolar interaction is the mechanism for complexation, the interaction would be expected to weaken with the addition of water to the buffer. The decrease in binding would be dependent on the properties of the analyte. It may be possible use this phenomenon to adjust the migration times of certain peaks into less cluttered areas of the electropherogram.

Conditions that achieved high efficiency and short analysis times were chosen for the development of the fingerprinting technique (described in section 3.2.8). For this reason a buffer with no water was used. The possibility of manipulating the separation through changes in polarity may be useful in further experiments that focus on particular components of PHOTOFRIN ${ }^{\circledR}$.

\subsubsection{Effect of $\mathrm{pH}$ and CAPS Concentration}

The buffer $\mathrm{pH}$ was kept high to ensure the solubility of PHOTOFRIN ${ }^{\circledR}$. This had the added benefit of maximizing the EOF. Changes in $\mathrm{pH}$ above 10 had little effect on migration times since the porphyrins should be completely deprotonated at this $\mathrm{pH}$. Most of the silanol groups on the capillary wall will be deprotonated as well, allowing little change in the EOF. 
The effect of different concentrations of CAPS was tested from 10 to $30 \mathrm{mM}$. The change in migration times was negligible. Therefore, a low buffer concentration was chosen to maximize the EOF and minimize the possibility of Joule heating.

\subsubsection{Detection}

Figure 3.2.5 compares electropherograms monitored by fluorescence and absorbance detectors. Hematoporphyrin was used as a standard to compare the two detection methods. The limits of detection (LOD) (i.e. three standard deviations of the background noise) were determined to be $34 \mathrm{ppb}$ and $45 \mathrm{ppb}$ for absorbance and fluorescence detection respectively. The relatively poor detection limit for fluorescence in this case is due to poor absorbance at the excitation wavelength $(488 \mathrm{~nm})$. The major absorption band for hematoporphyrin is at $400 \mathrm{~nm}$ with a less intense band at $490-510 \mathrm{~nm}^{(47)}$. A much better LOD would be expected if fluorescence was induced at the optimum wavelength.

The hematoporphyrin calibration curves for absorbance and fluorescence were found to be linear $\left(\mathrm{R}^{2}>0.999\right)$ up to $100 \mathrm{ppm}$. The percent weight of hematoporphyrin in PHOTOFRIN ${ }^{\circledR}$ was determined to be $(0.81 \pm 0.02) \%$ using these calibration curves.

The major difference between absorbance and fluorescence detection occurs in the oligomeric fraction. The relative intensity in the oligomer region is higher with absorbance detection than fluorescence because the oligomers undergo self quenching, decreasing the fluorescence yield. Since fewer compounds are detected, the fluorescence detection presents a clearer pattern, which is more useful in developing a fingerprint of the drug. Absorbance will give a better indication of the true amount of material in the oligomeric fraction. 

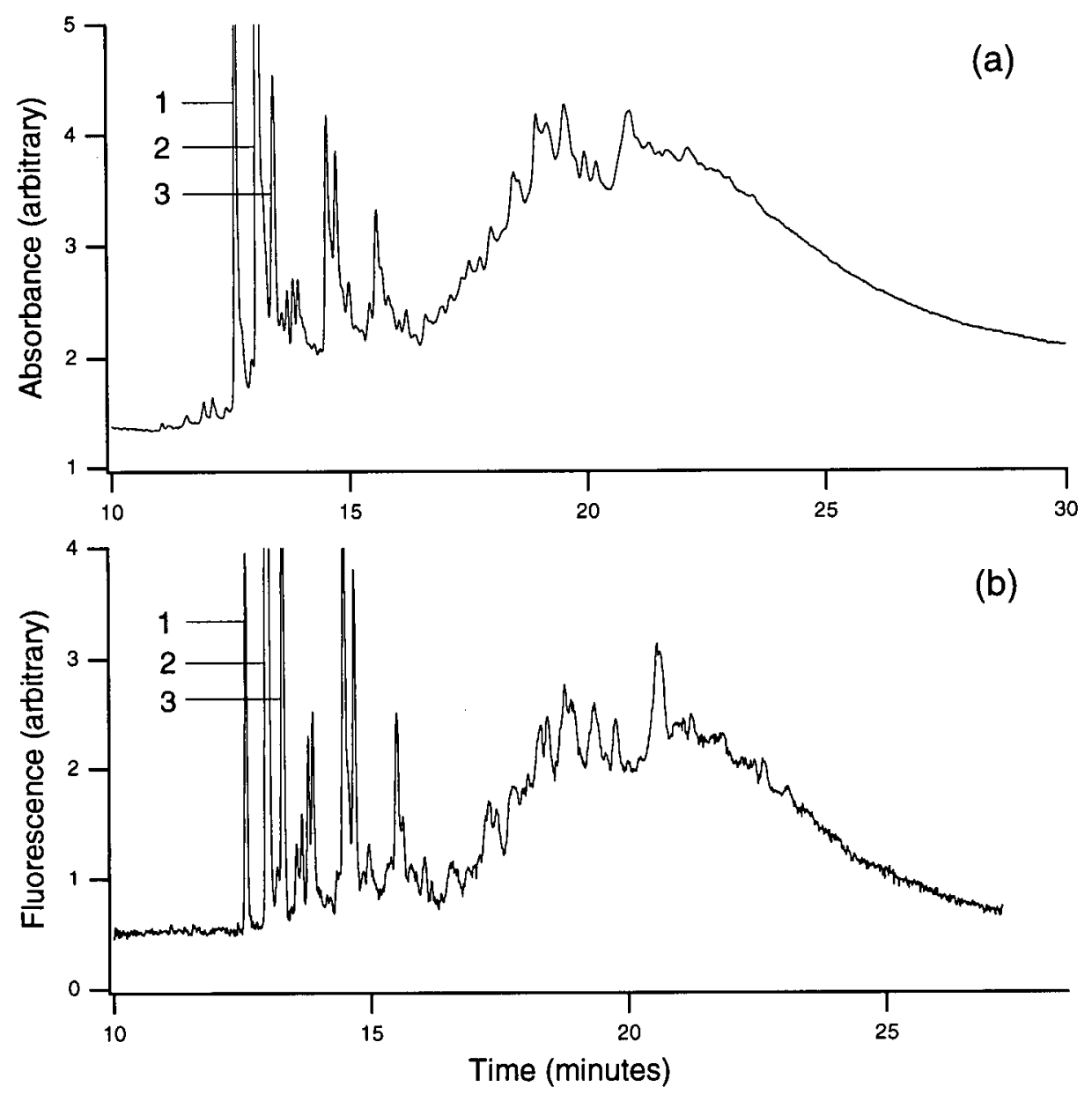

Figure 3.2.5 Comparison of (a) absorbance and (b) fluorescence detection for the nonaqueous CE separation of PHOTOFRINQ. The numbered peaks are: (1) PP, (2) HVD, and (3) HP.

There are approximately 50 partially resolved peaks observed with fluorescence detection compared to 60 with absorbance. This discrepancy is caused by the signal differences in the oligomer region. There are several early eluting peaks with efficiencies of approximately 200,000 theoretical plates. Each peak may represent individual or isomeric compounds. Most of the peaks in the oligomer region are much broader and not well resolved indicating that they may not be pure compounds. It can be inferred that PHOTOFRIN ${ }^{\circledR}$ is made up of at least 60 compounds with the actual total being much higher. 


\subsubsection{PHOTOFRIN ${ }^{\circledR}$ Fingerprint}

The problems in characterizing PHOTOFRIN ${ }^{\circledR}$ lie in its oligomeric component. As stated earlier there are at least 60 compounds in this region. It may be impossible or at least highly impractical to completely resolve all of them. Another approach is to qualitatively compare peaks, using the oligomer region of the electropherogram as a fingerprint. There are enough characteristic peaks in the electropherogram to make this possible. The fingerprint will be especially useful in the area of quality control. Although the individual identities of the peaks are unknown, it will be possible to qualitatively compare the peaks in the oligomer region. Figure 3.2.6 shows excellent reproducibility between batches of PHOTOFRIN ${ }^{\circledR}$. The potential of this method is illustrated in Figure 3.2.7, which shows the changes that occur in the oligomeric region when the drug is exposed to even minor stresses.

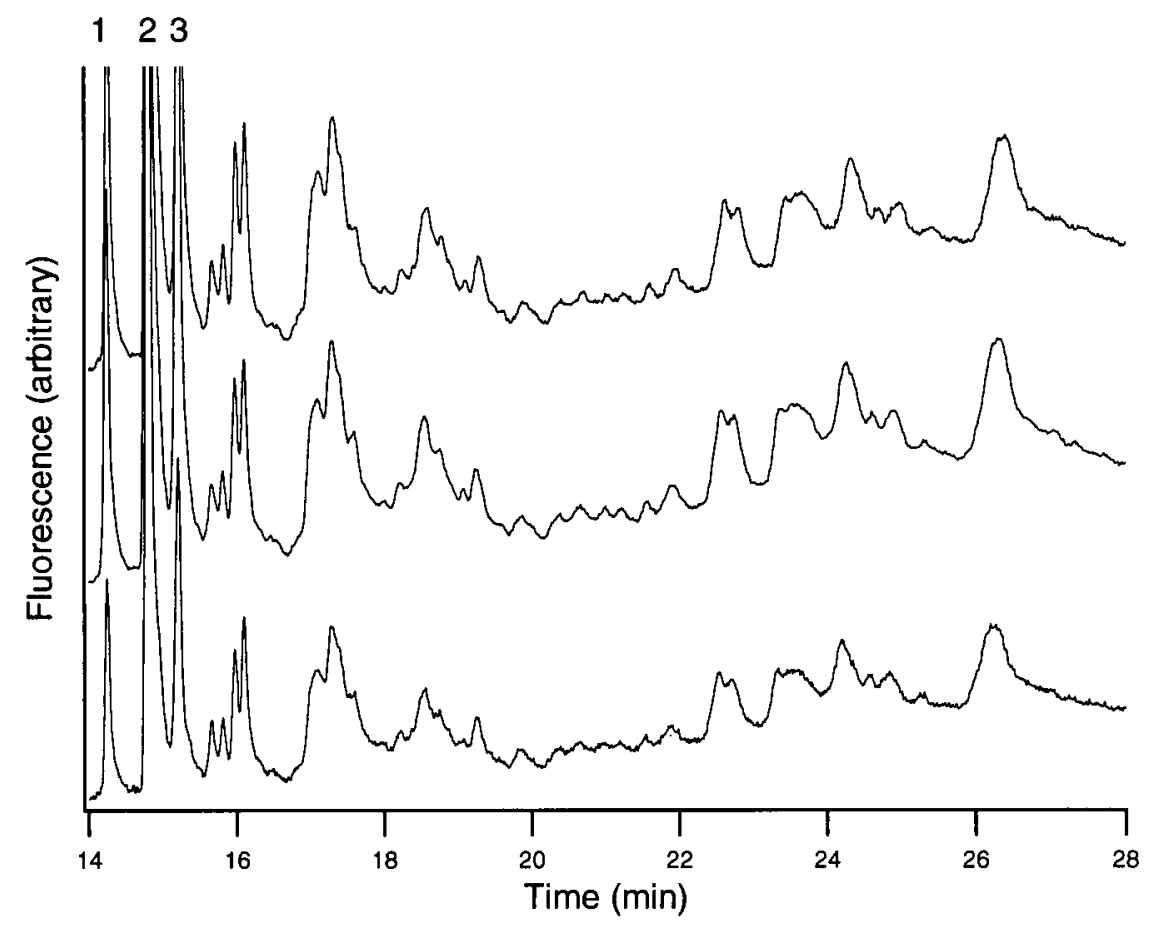

Figure 3.2.6 Electropherograms of three different batches of PHOTOFRIN ${ }^{\circledR}$. The numbered peaks are: (1) PP, (2) HVD, and (3.) HP. 


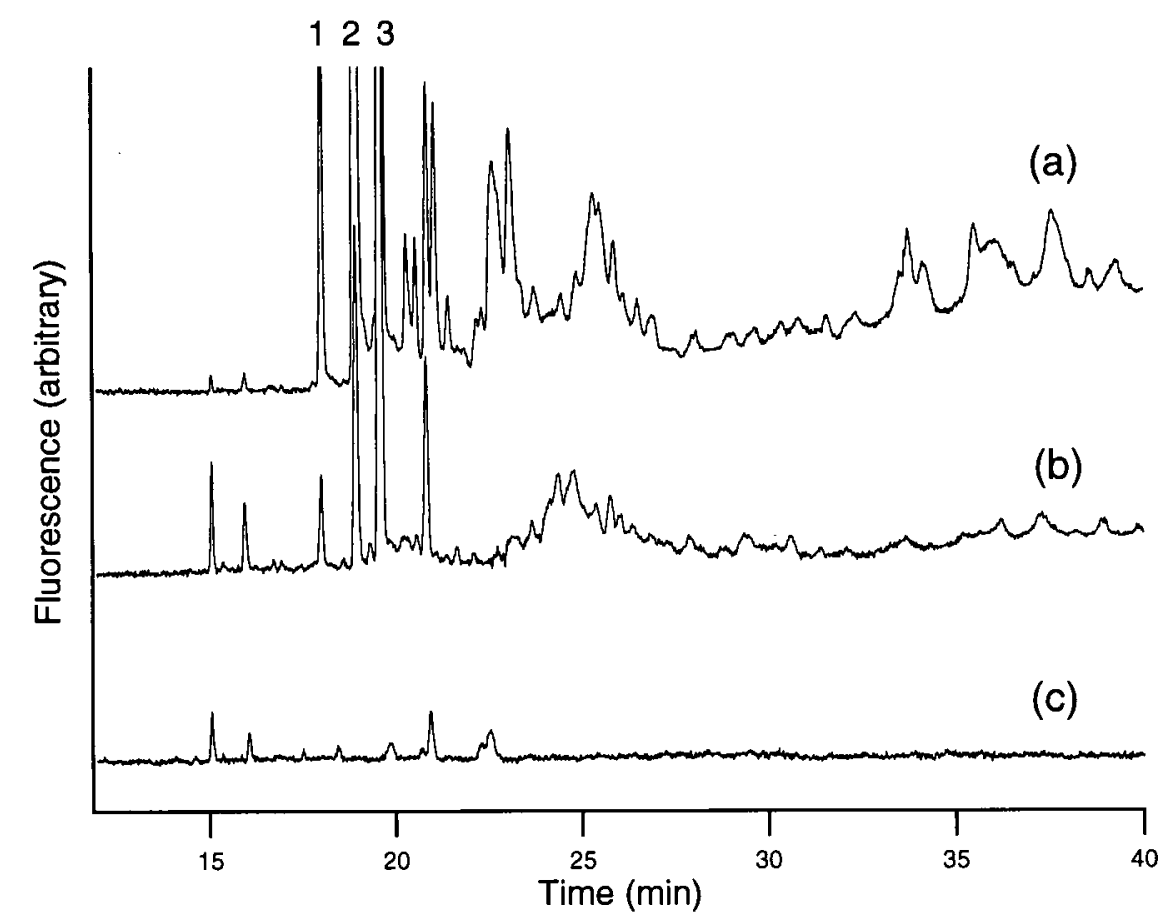

Figure 3.2.7 Qualitative comparisons of stressed PHOTOFRIN ${ }^{\circledR}$ samples. Electropherogram (a) is standard PHOTOFRIN ${ }^{\circledR}$. Electropherograms (b) and (c) are PHOTOFRIN ${ }^{\circledR}$ stressed in aqueous solution at $80^{\circ} \mathrm{C}$ and exposed to light for (b) one day and (c) four days. The numbered peaks are: (1) PP, (2) HVD, and (3) HP.

\subsubsection{Conclusions}

This chapter demonstrated an assay of a complex mixture of porphyrin monomers and oligomers using nonaqueous CE. Brij 35 was used as a complexation additive that both modified selectivity and improved efficiency by interacting with the analytes. The effect of the EOF and the water content of the buffer was discussed. A comparison between absorbance and fluorescence detection was also made. The complexity of the oligomeric region of the electropherogram provided enough detail to be used as a qualitative fingerprint that could be used to assess the composition of the mixture. 


\subsection{Quantitative Description of the Migration Behavior of Porphyrins in a Nonaqueous Capillary Electrophoresis System}

\subsubsection{Introduction}

The previous chapter discussed the CE assay of a complex mixture of porphyrin monomers and oligomers using a polyether surfactant in an nonaqueous buffer system. A better understanding of the role brij 35 plays in the separation would be useful in designing new assays. Unfortunately, the mixture discussed in chapter 3.2 is much too complicated to perform a quantitative study of the porphyrin-brij 35 binding. In this chapter a group of monomeric porphyrin acids are used to further study the brij 35 interactions. The conditions developed in chapter 3.2 will be used so that the findings made here can be extended to the PHOTOFRIN® assay.

The development of an assay for monomeric porphyrins is important in itself. The uses of porphyrin derived compounds in biological systems range from photoreceptors in plants and bacteria $^{(53)}$ to the main prosthetic group in animal heme proteins ${ }^{(54)}$. The metabolic history of these molecules in humans can be tracked by monitoring the pigments in the urine, blood and feces ${ }^{(55)}$. New analytical methods which can readily assay porphyrins such as uroporphyrin and coproporphyrin are important for their possible application in monitoring a number of diseases including porphyria and jaundice ${ }^{56)}$. A CE separation of monomeric porphyrin acids has been demonstrated previously using SDS as an additive in aqueous solution ${ }^{(50)}$. However, porphyrins and their oligomers tend to aggregate in aqueous solutions, which often affects the migration time and separation efficiency. Therefore, in many cases nonaqueous solvents are preferable.

There are three major steps in developing a model for an untested complexation additive in a CE separation. The first step is to propose a reasonable model to describe the system. The second step is to test the assumptions made in deriving the equations to ensure the model is useful in describing the experimental results. Proper experimental conditions must be chosen to 
minimize errors introduced during the experiment. The third step involves making estimates for the constants in the equations so the model can be used to predict experimental results under different conditions. It may also be possible to deduce some of the physical or chemical properties of both the analyte and the additive from these parameters.

\subsubsection{Background}

The simplest and most commonly encountered analyte-additive equilibrium in CE has a stoichiometry of $1: 1$. The probability of two analyte molecules complexing with one additive is small because the concentration of analyte is often much smaller than that of the additive. As discussed in chapter 1.3 , complexation of two additive molecules with one analyte can occur if there is more than one binding site on the analyte. A model for this system will be initially developed based on the assumption that binding takes place with a $1: 1$ analyte-additive stoichiometry. This assumption will be tested using the methods described in section 1.3.5. Equation 1.3.14 describes the effect of a complexation additive, that interacts with the analyte at a 1:1 stoichiometry, on the net mobility of an analyte:

$$
\left(v \mu_{e p}^{\mathrm{A}}-\mu_{\mathrm{ep}, \mathrm{A}}\right)=\frac{\left(\mu_{\mathrm{ep}, \mathrm{AC}}-\mu_{\mathrm{ep}, \mathrm{A}}\right) \mathrm{K}_{\mathrm{AC}}[\mathrm{C}]}{1+\mathrm{K}_{\mathrm{AC}}[\mathrm{C}]}
$$

where $v$ is a correction factor that normalizes the mobility to the ideal case where the additive does not change the properties of the buffer, $\mu_{\mathrm{ep}}^{\mathrm{A}}$ is the net electrophoretic mobility of the analyte, $\mu_{\mathrm{ep}, \mathrm{AC}}$ is the electrophoretic mobility of the analyte-additive complex, $\mu_{\mathrm{ep}, \mathrm{A}}$ is the electrophoretic mobility of the uncomplexed analyte, $\mathrm{K}_{\mathrm{AC}}$ is the binding constant, and $[\mathrm{C}]$ is the additive concentration.

\subsubsection{Experimental}

Separations were performed on a Beckman P/ACE 5000 automated CE system (Beckman 
Instruments Inc., Mississauga, Ont., Canada). Uncoated fused silica capillaries (Polymicro Technologies, Phoenix, AZ) were used with inner diameters of $50 \mu \mathrm{m}$, outer diameters of 375 $\mu \mathrm{m}$, and lengths of $57 \mathrm{~cm}$. The length from the injection end to the detector (effective length) was $50 \mathrm{~cm}$. New capillaries were rinsed for 20 minutes with aqueous $1 \mathrm{M}$ sodium hydroxide (BDH Inc., Toronto, Ont., Canada) followed by methanol for 5 minutes and separation buffer for 20 minutes. The capillary was then allowed to equilibrate overnight before being used. A 15 minute rinse using the separation buffer was performed before the separation if the buffer composition was different from the previous run. If the conditions were unchanged from the previous run, a 2 minute rinse was used. The sample was introduced using a three second injection at $-10 \mathrm{kV}$. Separations took place at a potential of $-30 \mathrm{kV}$ and the temperature was controlled at $20^{\circ} \mathrm{C}$. Fluorescence was induced with the $488 \mathrm{~nm}$ line of a $4 \mathrm{~mW}$ argon ion laser (Beckman). Emission was monitored at $620 \mathrm{~nm}$ with a Beckman fluorescence detector. Data were collected and processed using System Gold software (Beckman) and a 386 PC computer. The buffer viscosity was measured by injecting ( 3 seconds at $-10 \mathrm{kV}$ ) a sample plug onto the capillary and measuring the time needed to push the plug to the detector with a constant pressure (20 psi). All regressions were performed manually in Microsoft Excel 5.0 using the least squares variance-covariance method.

Stock solutions of $30 \mathrm{mM}$ 3-[cyclohexylamino]-1-propanesulfonic acid (CAPS) (Sigma, St. Louis, MO, U.S.A.) and $200 \mathrm{mM}$ brij 35 (Aldrich, Inc., Milwaukee, WI, U.S.A.) were prepared in HPLC grade methanol (Fisher Scientific, Nepean, Ont., Canada). The pH of the CAPS stock solution was adjusted to 10.5 with $0.2 \mathrm{M}$ sodium hydroxide in methanol before the solution was made up to its final volume. The $\mathrm{pH}$ of the final solution was then confirmed. This is not a measurement of $\mathrm{pH}$ in the conventional sense but was used to ensure the buffer is prepared reproducibly. The appropriate volumes of stock solutions were mixed with methanol to give separation buffers containing $20 \mathrm{mM}$ CAPS $(\mathrm{pH}=10.5)$ and $0-40 \mathrm{mM}$ brij 35 . The 
porphyrin standard mixture (Porphyrin Products, Logan, Utah, U.S.A.) contained 10 nanomoles each of mesoporphyrin, coproporphyrin, pentacarboxylporphyrin, hexacarboxylporphyrin, heptacarboxylporphyrin and uroporphyrin. The standard solution was prepared by dissolving the mixture in $1.00 \mathrm{~mL}$ of $1 \mathrm{M}$ aqueous ammonia to give a concentration of $10^{-5} \mathrm{M}$. This standard solution was injected directly onto the capillary without further dilution.

\subsubsection{The Separation}

Figure 3.3.1 shows the structures of the porphyrins being separated in this study. Except for mesoporphyrin, the compounds studied in this experiment are of the type I series porphyrins. Type I series porphyrins have four propyl carboxylic acids and zero to four acetyl carboxylic acids. Mesoporphyrin is not a member of this series and was added as a representative dicarboxyl porphyrin.

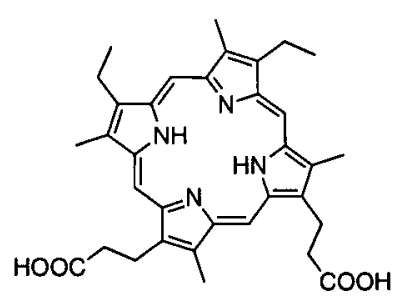

1

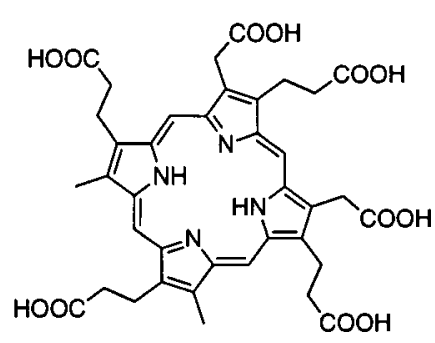

4

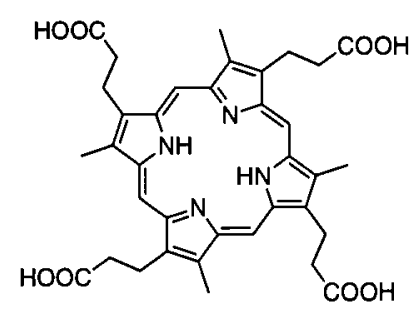

2

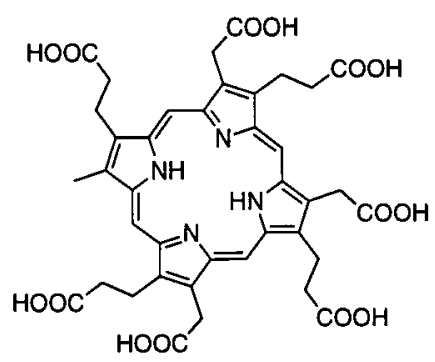

5

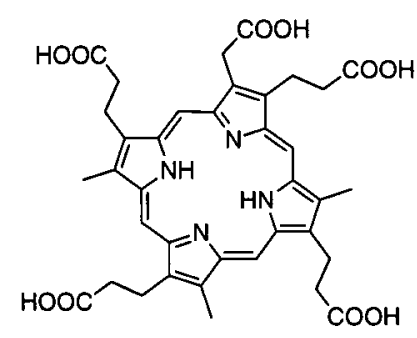

3

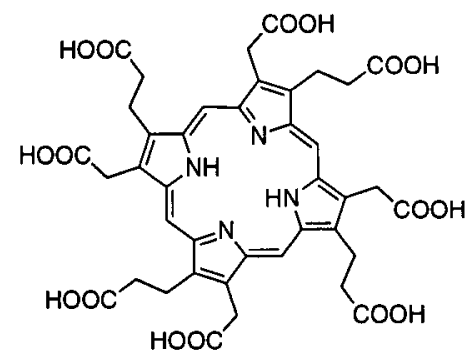

6

Figure 3.3.1 The structures of the porphyrin acids studied: 1 . mesoporphyrin, 2. coproporphyrin, 3. pentacarboxylporphyrin, 4. hexacarboxylporphyrin, 5 . heptacarboxylporphyrin, and 6. uroporphyrin. 


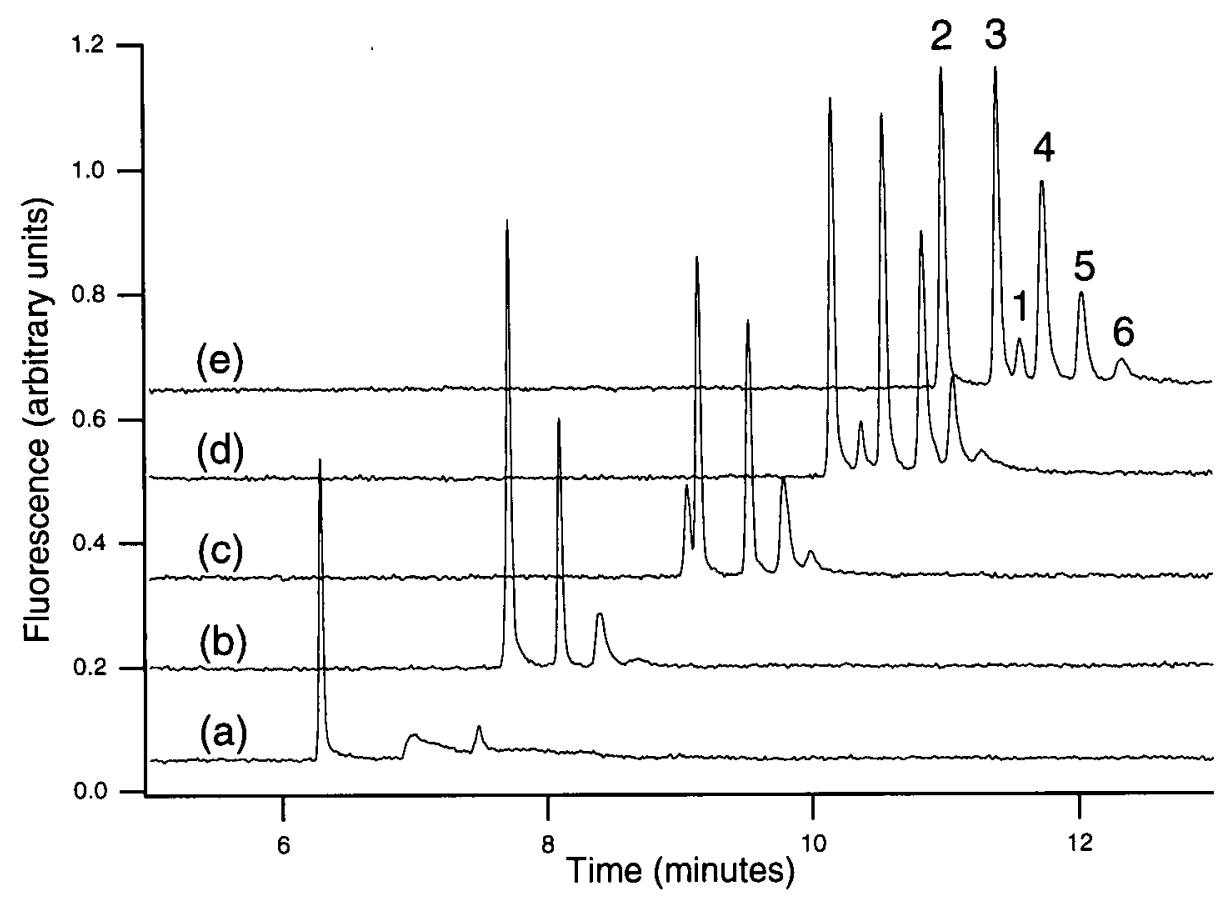

Figure 3.3.2 Electropherograms of the six porphyrin acids at different brij 35 concentrations. The concentrations of brij 35 are: (a) $0 \mathrm{mM}$, (b) 5 $\mathrm{mM}$, (c) $15 \mathrm{mM}$, (d) $25 \mathrm{mM}$, and (e) $35 \mathrm{mM}$. The peaks are numbered according to Figure 3.3.1.

Figure 3.3.2 shows electropherograms of the porphyrin standards at different brij 35 concentrations. The peaks were identified using coinjections of the individual porphyrins with the standard solution. The differences in fluorescence response between the porphyrins are largely due to differences in their molar absorption coefficient at $488 \mathrm{~nm}$. The separation conditions were similar to those developed in chapter 3.2 in order to better understand the brij 35-porphyrin interactions taking place in the PHOTOFRIN ${ }^{\circledR}$ assay. Methanol was chosen as a solvent to decrease molecular aggregation as well as increase the range of conditions under which the porphyrins are soluble. Brij 35 was chosen because of its high solubility in methanol as well as its ability to undergo dipole interactions. Although brij 35 is a surfactant, it will not form micelles in pure methanol. This is because the hydrophobic interactions that cause micelles to form in water are much weaker in methanol ${ }^{(20,57)}$. The porphyrins should complex with the polyether component of the brij 35 molecule through dipole interactions.

The potential applied to the capillary was $-30 \mathrm{kV}$ (i.e. negative at the inlet). This is 
necessary because the EOF under these conditions was very small. The $\mu_{\mathrm{eo}}$ was determined to be $<10^{-6} \mathrm{~cm}^{2} \mathrm{~V}^{-1} \mathrm{~s}^{-1}$ using benzene as a marker. The negligible EOF allows the electrophoretic mobility of the analyte to be calculated directly from the migration times.

The viscosity correction factor $(v)$ was found to be linear $\left(v=6.805 \mathrm{M}^{-1} \times[\mathrm{Brij} 35]+\right.$ $1.005 ; \mathrm{R}^{2}=0.970$ ) over the range of additive concentrations used in this study. This relationship shows that there was a $28 \%$ increase in viscosity over the range of the brij 35 concentrations studied, demonstrating the importance of the viscosity correction.

With appropriate brij 35 concentrations, baseline separation of all six porphyrins can be achieved in less than 15 minutes. This is largely due to the high efficiency of the separation ( $>300,000$ plates), and the effect of the analyte-additive interaction. The difference in mobilities between the free analytes is not as large as anticipated even though the number of carboxylic acids varied from 2 to 8 for the six porphyrin acids studied. The migration order was also different than would be predicted based on the number of ionizable groups. The possible explanations for these phenomena will be discussed in section 3.3.7. There is a clear trend of increasing migration times as the brij 35 concentration increased. This was expected because the free analytes have larger electrophoretic mobilities than the complex molecules. The viscosity of the buffer also increased with increased additive concentrations, which would also decrease analyte mobility.

\subsubsection{Testing the Model}

Ensuring that the chosen model, in this case a 1:1 complexation, accurately describes the analyte-additive interaction is a crucial step in understanding the separation process in CE. Many studies spend little or no attention to this step. As discussed in section 1.3.5, not all of the linear transformations are equal in their ability to detect deviations from the $1: 1$ model and $x$ - 
164.

reciprocal plots should be used for this purpose. Figure 3.3.3 shows the $x$-reciprocal plots of the six porphyrin acids studied. The equation for the $x$-reciprocal plot is given in Table 2.1.1.

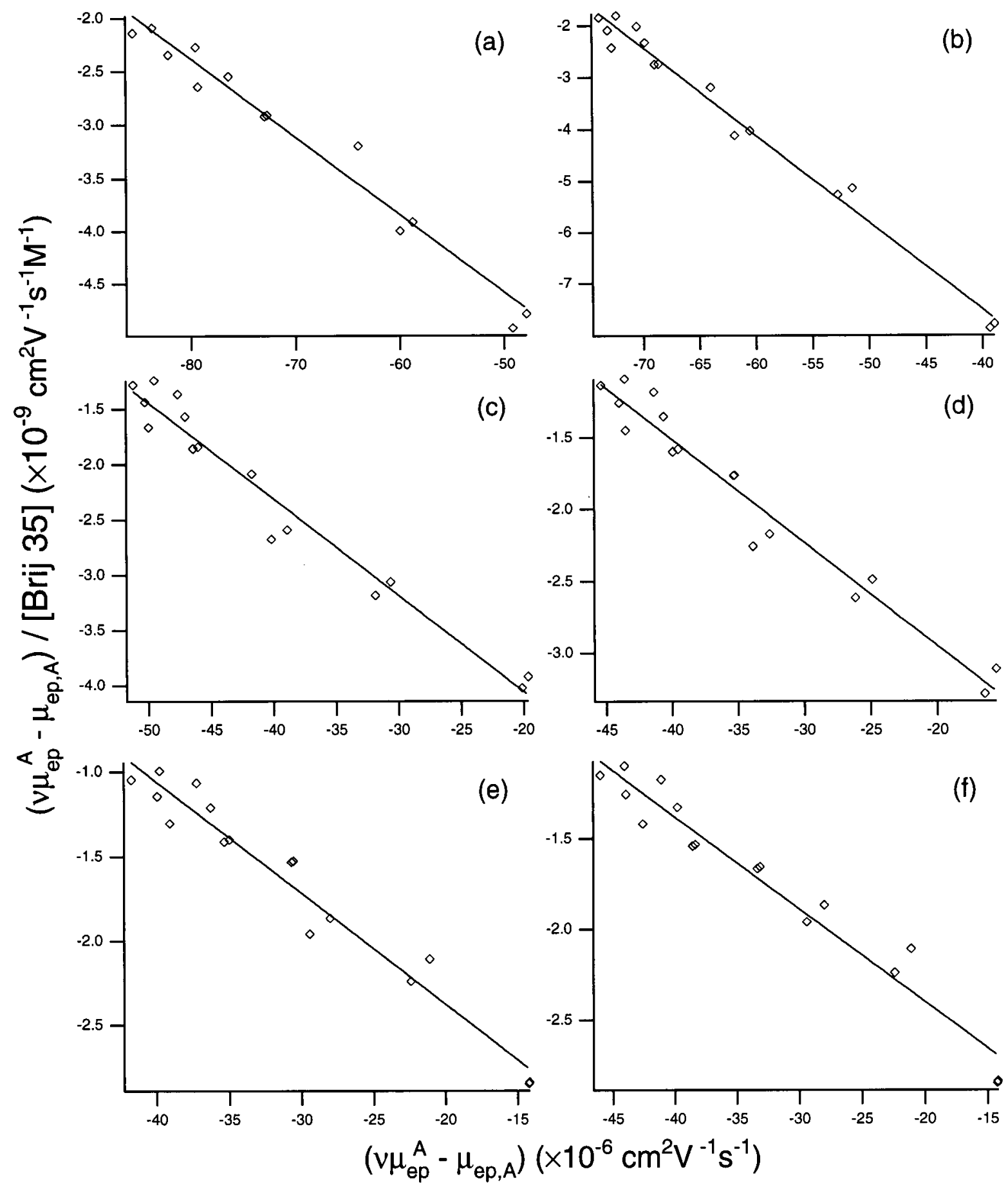

Figure 3.3.3 The $x$-reciprocal plots of the six porphyrin acids: (a) mesoporphyrin, (b) coproporphyrin, (c) pentacarboxylporphyrin, (d) hexacarboxylporphyrin, (e) heptacarboxylporphyrin, and (f) uroporphyrin.

The $x$-reciprocal plots for the six porphyrins studied shown in Figure 3.3.3 are all linear, 
supporting the hypothesis that a 1:1 equilibrium model can be used to describe the brij 35 porphyrin interaction. There is a fair amount of scatter but all the plots are clearly linear with $\mathrm{R}^{2}$ $>0.95$. It should be noted that visual inspections of these plots should be used since data with obvious nonlinearity can often give a high value for $\mathrm{R}^{2}$. The fact that brij 35 interacts with the porphyrin acids with a 1:1 stoichiometry may seem surprising considering the multiple sites on both molecules that can take part in dipole interactions. There are 23 ethers on brij 35 capable of forming dipole interactions. Because its size, the brij 35 molecule can wrap around the porphyrins, interacting with all of the available binding sites. This prevents the attachment of a second additive molecule. As stated in section 1.3.5, the observation of a 1:1 stoichiometry over a certain additive concentration range does not preclude the formation of higher order interactions at higher additive concentrations. Therefore, brij 35-porphyrin complexes with different stoichiometries may form at additive concentrations above $40 \mathrm{mM}$.

Another consideration in designing a binding experiment is the range over which data should be collected. It was shown in chapter 2.2 that the error in the binding constant estimate will be minimized when data are collected for additive concentrations where the fraction of analyte complexed ranges from approximately 0.5 to 0.95 . The fraction of the porphyrins that are complexed at the lowest additive concentration ranged from 0.2 to 0.46 . The fraction of the porphyrins that are complexed at the highest additive concentration ranged from 0.67 to 0.87 . Data was collected that covers the central to upper portions of the binding isotherms, thereby minimizing the error in the estimated constants.

\subsubsection{Estimating the Binding Constant and Complex Mobility}

Once the validity of the model has been confirmed, the values of the binding constant and the mobility of the analyte-additive complex can be estimated. The most commonly used methods involve measuring the electrophoretic mobility of the free analyte directly, followed by 
either a nonlinear regression according to eq 3.3 .1 or one of the three linear plotting methods shown in Table 2.1.1. With the proper choice of experimental conditions and ideal weighting of the data, each of these relationships should give similar results (see chapters 2.2 and 2.3). In practical cases, the nonlinear regression should give the most reliable estimates for the constants.

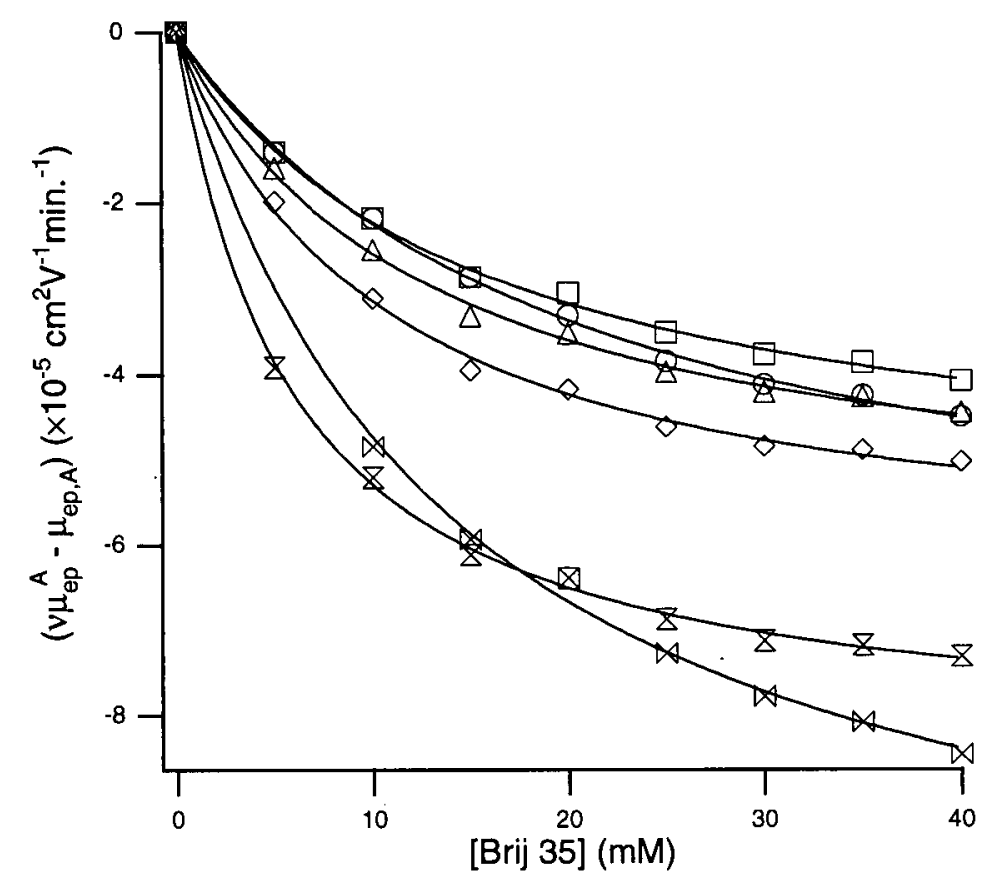

Figure 3.3.4 Nonlinear fits (made according to eq 3.3.1) of the binding isotherms of the six porphyrin acids: mesoporphyrin $(\bowtie)$, coproporphyrin $(\varangle)$, pentacarboxylporphyrin $(\diamond)$, hexacarboxylporphyrin $(\triangle)$, heptacarboxylporphyrin ( $\square)$, and uroporphyrin. (O).

Figure 3.3.4 compares the curves estimated using the nonlinear regression to the experimental data. These are the binding isotherms for the porphyrin-brij 35 interactions, showing the change in mobility caused by the complexation at different additive concentrations. Tables 3.3.1 and 3.3.2 compare the values of $\mathrm{K}_{\mathrm{AC}}$ and $\mu_{\mathrm{ep}, \mathrm{AC}}$ obtained using the nonlinear regression to estimates made using the three linear plotting methods. The values calculated using the nonlinear regression and the three linear plotting methods are similar but there are differences in the estimated constants and the errors associated with them, demonstrating that the different calculation methods do not give identical results. 
Table 3.3.1 Binding constants $\left(\mathrm{M}^{-1}\right)$ for the porphyrin-brij 35 complexes. The error limits represent the $95 \%$ confidence intervals. All of the methods were weighted according to the functions given in Table 2.1.1.

\begin{tabular}{lcccc}
\hline & $x$-Reciprocal & $y$-Reciprocal & $\begin{array}{c}\text { Double } \\
\text { Reciprocal }\end{array}$ & $\begin{array}{c}\text { Nonlinear } \\
\text { Regression }\end{array}$ \\
\hline Mesoporphyrin & $70 \pm 10$ & $73 \pm 3$ & $73 \pm 3$ & $72 \pm 2$ \\
Coproporphyrin & $166 \pm 14$ & $169 \pm 9$ & $169 \pm 9$ & $169 \pm 7$ \\
Pentacarboxylporphyrin & $92 \pm 13$ & $97 \pm 6$ & $97 \pm 6$ & $96 \pm 4$ \\
Hexacarboxylporphyrin & $74 \pm 12$ & $78 \pm 5$ & $78 \pm 5$ & $78 \pm 3$ \\
Heptacarboxylporphyrin & $62 \pm 11$ & $66 \pm 4$ & $66 \pm 4$ & $66 \pm 3$ \\
Uroporphyrin & $47 \pm 8$ & $50 \pm 2$ & $50 \pm 2$ & $49 \pm 1$ \\
\hline
\end{tabular}

Table 3.3.2 Electrophoretic mobilities of the uncomplexed porphyrins and the porphyrin-brij 35 complexes. The error limits represent the $95 \%$ confidence intervals. All of the methods were weighted according to the functions given in Table 2.1.1.

\begin{tabular}{lccccc}
\hline & $\begin{array}{c}\mu_{\mathrm{ep}, \mathrm{A}} \\
\left(\times 10^{-4} \mathrm{~cm}^{2} \mathrm{~V}^{-1} \mathrm{~s}^{-1}\right)\end{array}$ & \multicolumn{4}{c}{$\begin{array}{c}\mu_{\mathrm{ep}, \mathrm{Ac}} \\
\left(\times 10^{-4} \mathrm{~cm}^{2} \mathrm{~V}^{-1} \mathrm{~s}^{-1}\right)\end{array}$} \\
\cline { 3 - 6 } & & $x$-Reciprocal & $y$-Reciprocal & $\begin{array}{c}\text { Double } \\
\text { Reciprocal }\end{array}$ & $\begin{array}{c}\text { Nonlinear } \\
\text { Regression }\end{array}$ \\
\hline Mesoporphyrin & $2.52 \pm 0.01$ & $1.4 \pm 0.3$ & $1.39 \pm 0.05$ & $1.39 \pm 0.09$ & $1.39 \pm 0.02$ \\
Coproporphyrin & $2.52 \pm 0.01$ & $1.7 \pm 0.2$ & $1.68 \pm 0.02$ & $1.68 \pm 0.06$ & $1.68 \pm 0.01$ \\
Pentacarboxylporphyrin & $2.23 \pm 0.01$ & $1.6 \pm 0.4$ & $1.58 \pm 0.03$ & $1.58 \pm 0.07$ & $1.58 \pm 0.05$ \\
Hexacarboxylporphyrin & $2.12 \pm 0.01$ & $1.5 \pm 0.4$ & $1.52 \pm 0.03$ & $1.52 \pm 0.06$ & $1.52 \pm 0.06$ \\
Heptacarboxylporphyrin & $2.04 \pm 0.01$ & $1.5 \pm 0.4$ & $1.47 \pm 0.04$ & $1.47 \pm 0.06$ & $1.48 \pm 0.07$ \\
Uroporphyrin & $2.04 \pm 0.01$ & $1.3 \pm 0.3$ & $1.35 \pm 0.05$ & $1.35 \pm 0.06$ & $1.35 \pm 0.07$ \\
\hline
\end{tabular}

Figure 3.3.5 (a) compares the mobilities predicted by the constants listed in Tables 3.3.1 and 3.3.2 to the experimentally measured mobilities. The viscosity correction factor is linearly related to the additive concentration, and can be accounted for in the predicted curve. Because the electroosmotic flow is negligible in this system, the theoretical migration times can be calculated directly from $\mu_{\mathrm{ep}}^{\mathrm{A}}$. The calculated values are compared to the experimental data in Figure 3.3.5 (b). Curves such as those shown in Figure 3.3.5 are very useful in determining the optimum additive concentration for a CE separation. 
168.
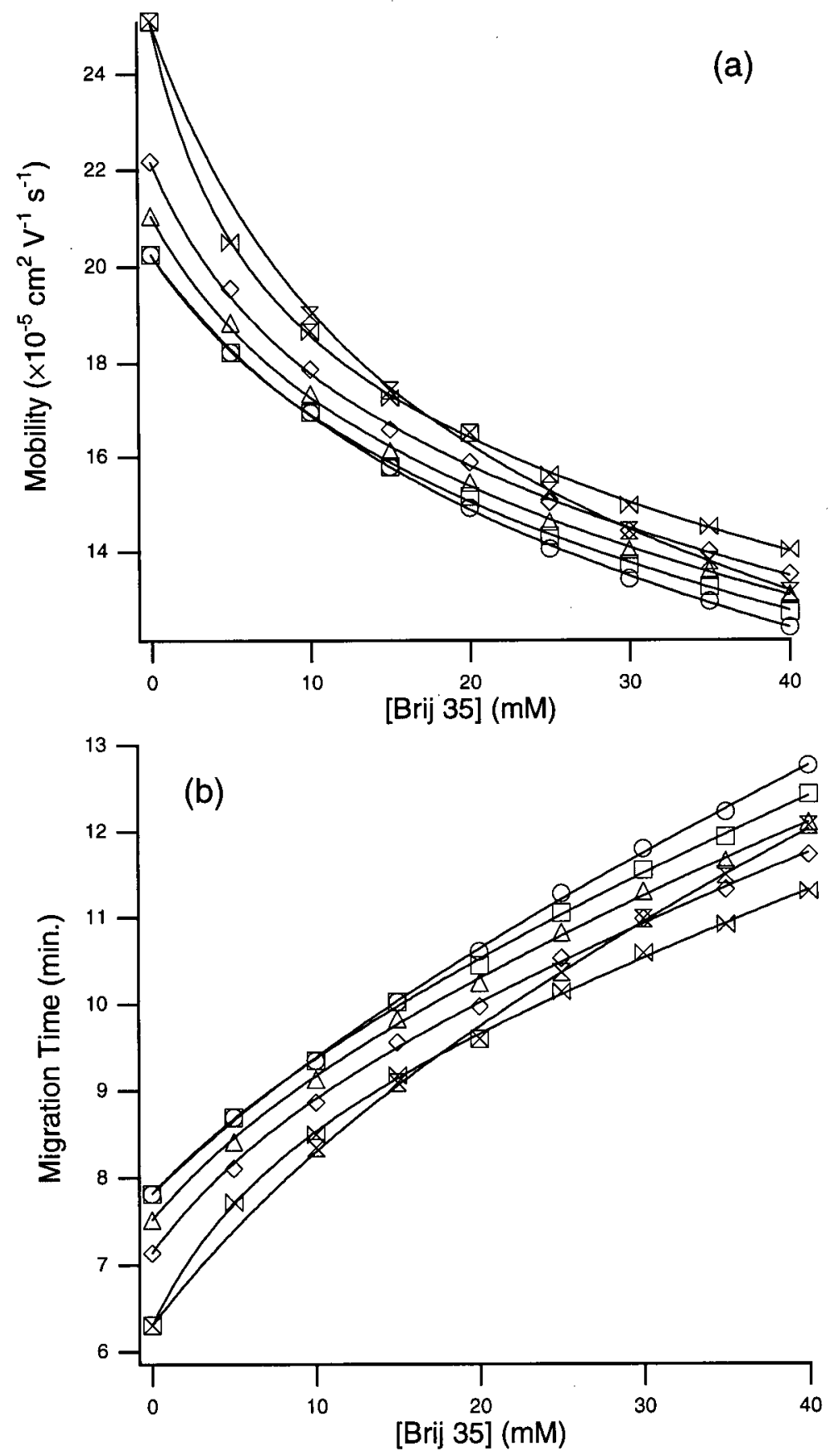

Figure 3.3.5 Comparison of the (a) mobilities and (b) migration times estimated using the constants in Tables 3.3.1 and 3.3.2, and the correlation between the viscosity correction factor and the brij 35 concentration to the experimental data for: mesoporphyrin $(\bowtie)$, coproporphyrin $(\varangle)$, pentacarboxylporphyrin $(\diamond)$, hexacarboxylporphyrin $(\triangle)$, heptacarboxylporphyrin $(\square)$, and uroporphyrin. $(O)$.

3.3.7 Trends in $\mathrm{K}_{\mathrm{AC}}, \mu_{\mathrm{ep}, \mathrm{A}}$, and $\mu_{\mathrm{\theta p}, \mathrm{AC}}$

The binding constants of the porphyrin-brij 35 are listed in Table 3.3.1. The free energies 
of the porphyrin-brij 35 interactions can be calculated using the equation: $\Delta \mathrm{G}^{\circ}=-\mathrm{RT} \ln \mathrm{K}$, where $\mathrm{G}^{\mathrm{o}}$ is the Gibb's free energy, $\mathrm{R}$ is the gas constant and $\mathrm{T}$ is the absolute temperature. The free energies of the studied interactions range from -9.5 to $-12.5 \mathrm{~kJ} / \mathrm{mol}$. These values support the premise that binding takes place through dipole interactions, which typically range from -4 to -13 $\mathrm{kJ} / \mathrm{mol}^{(58)}$.

Figure 3.3.6 shows the trends in the binding constants of the different porphyrin acids. Two factors need to be considered in explaining the trends of $\mathrm{K}_{\mathrm{AC}}$ values: 1) the number of binding sites available on the porphyrin, and 2) how tightly the solvation shell is bound to the porphyrins.

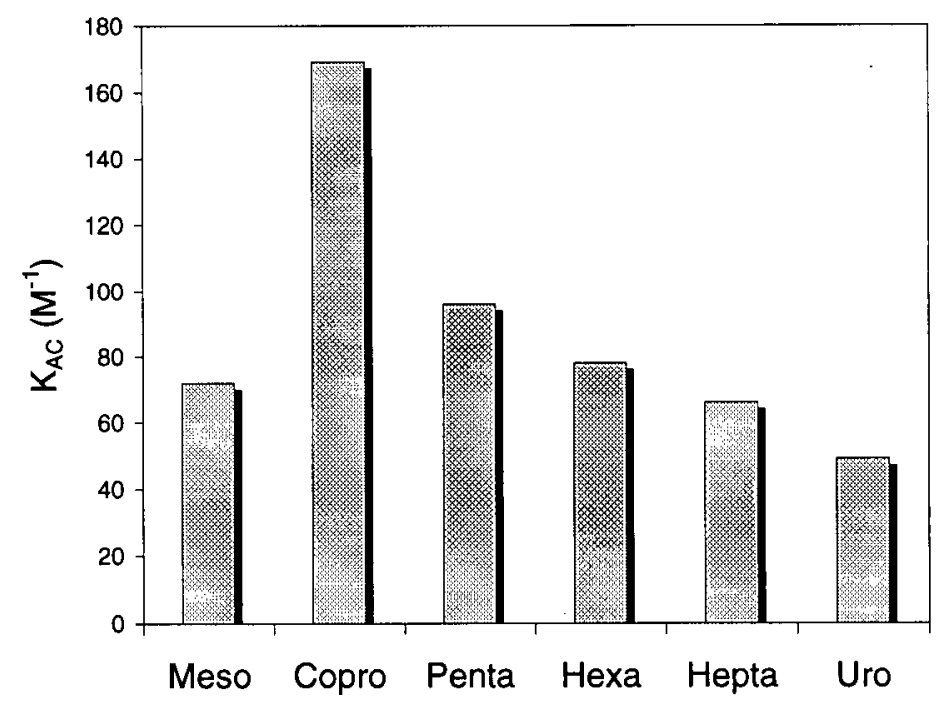

Figure 3.3.6 Binding constants of the porphyrin-brij 35 complexes estimated using nonlinear regression.

Because brij 35 has many sites that can that can take part in dipole interactions, it probably binds all of the available binding sites on the porphyrin. Therefore, porphyrins with more binding sites should undergo stronger interactions and have higher binding constants.

The strength of the interactions between an analyte and its solvation shell can have a major effect on its interaction with an additive ${ }^{(59)}$. More tightly bound solvation shells prevent 
the additive from getting close to the analyte, thereby decreasing $\mathrm{K}_{\mathrm{AC}}$. Molecules with higher polarities tend to have more tightly bound solvation shells. The trend of decreasing $\mathrm{K}_{\mathrm{AC}}$ values with increasing acetyl acids in the type I porphyrins (coproporphyrin to uroporphyrin) can largely be attributed to solvation effects. The strength of the solvent interaction increases as the polarity of the porphyrin increases, resulting in lower $\mathrm{K}_{\mathrm{AC}}$ values. The increased number of acetyl acids does not provide extra binding sites because they are sterically shielded by the larger propyl acids, suggesting that the porphyrin-brij 35 interactions take place through the propyl acids. The decrease in $\mathrm{K}_{\mathrm{AC}}$ from coproporphyrin to mesoporphyrin can be explained by the lower number of binding sites (propyl acids) available on mesoporphyrin.

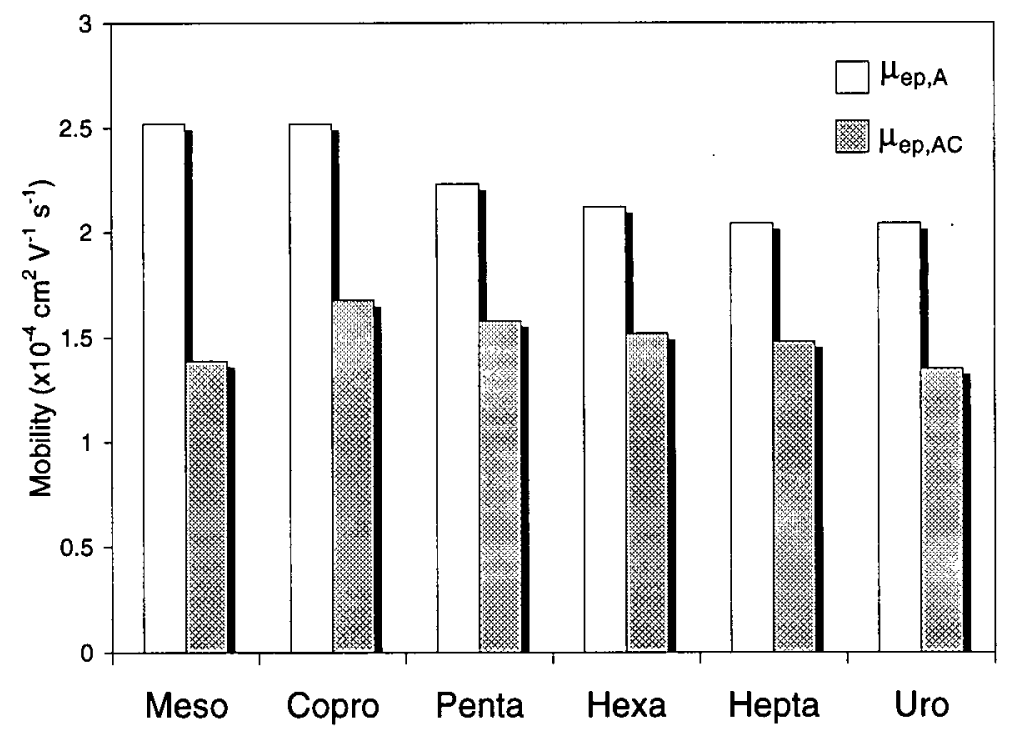

Figure 3.3.7 Electrophoretic mobilities of the uncomplexed porphyrins $\left(\mu_{\mathrm{ep}, \mathrm{A}}\right)$ and the porphyrin-brij 35 complexes $\left(\mu_{\mathrm{ep}, \mathrm{AC}}\right)$.

Figure 3.3.7 shows the electrophoretic mobilities of the uncomplexed porphyrins and the porphyrin-brij 35 complexes. The mobility of an analyte is determined by its charge and cross sectional area as well as the viscosity of the buffer. Because the mobilities listed in Table 3.3.2 have been normalized to account for changes in viscosity, the size and charge of the analytes must be used to explain the trends in Figure 3.3.7.

The mobilities of the uncomplexed and complexed type I porphyrins (coproporphyrin to 
uroporphyrin) decreased as the number of acetyl acids increased. We had expected porphyrins with higher numbers of acids to have a higher charge and therefore a higher mobility. The similar mobilities of the type I porphyrins suggest that they all bear a similar charge. The acetyl acids probably remain protonated because they are sterically shielded by the larger propyl acids. The protonated acetyl acids may also be stabilized by forming hydrogen bonds with the propyl acids. If the propyl acids are completely deprotonated, all of the type I porphyrins would then bear a -4 charge, leaving size as the determining factor in the mobilities. Clearly, the size of the porphyrins increases with increased numbers of acetyl acids (see Figure 3.3.1), decreasing the mobility.

This explains the observed trend for the type I porphyrins. However, incorporating mesoporphyrin into this hypothesis is slightly more complicated. Mesoporphyrin has two propyl acids and should therefore bear a -2 charge, making it much slower than the other porphyrins. Figure 3.3.7 shows that this is not the case. The higher than expected mobility for mesoporphyrin can be explained by its orientation in the buffer. All of the porphyrins are disc shaped. Mesoporphyrin has both of its charges on one side of the ring, causing this side to be pulled towards the positive electrode, allowing the porphyrin disc to move through the buffer edge on (see Figure 3.3.8). The -4 charge on the type I porphyrins is distributed evenly around the ring. This charge distribution forces the type I porphyrins to move through the buffer with the face of the disc perpendicular to the direction of motion. The orientation of mesoporphyrin in the buffer will obviously result in a much smaller resistance cross section than the other porphyrins, thereby giving a higher than expected mobility. This rationale also explains the larger difference in mobility mesoporphyrin exhibits between its free and complexed forms. The mesoporphyrin-brij 35 complex is more spherical than the free analyte because the brij 35 molecule wraps around mesoporphyrin, increasing the resistance cross section (see Figure 3.3.8 (b)). Because of their orientation perpendicular to the electric field, the resistance cross sections 
of the type I porphyrins will not increase as much as mesoporphyrin on complexation with brij 35.

(a)
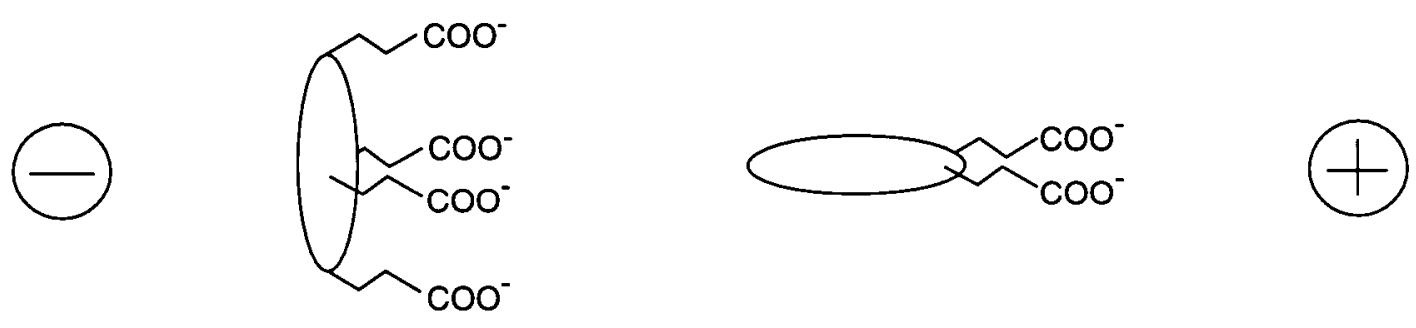

(b)
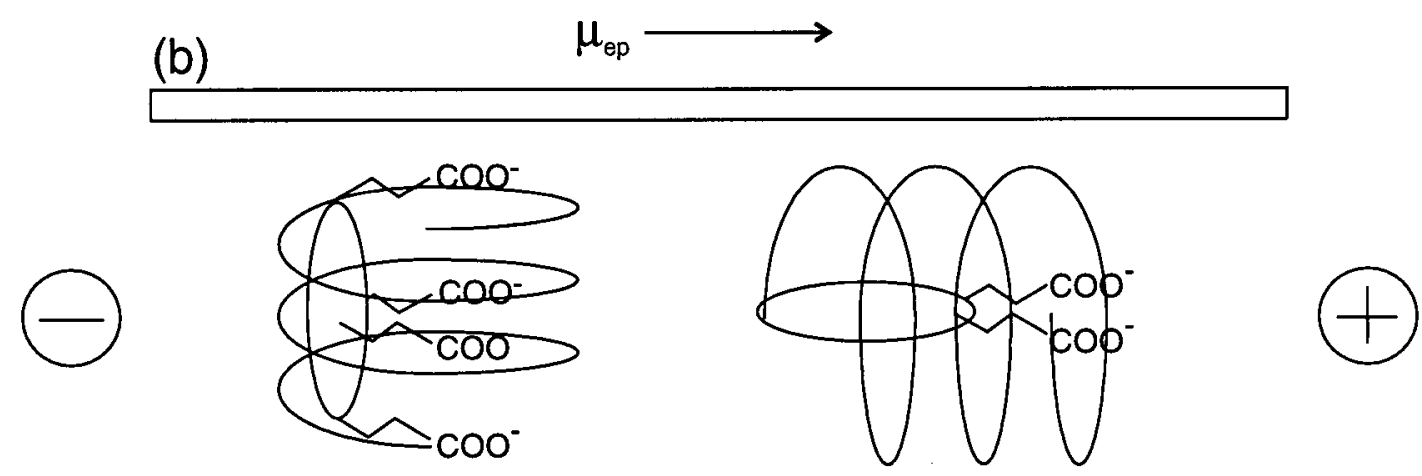

Type I Porphyrins

Mesoporphyrin

Figure 3.3.8 Preferred orientations of (a) uncomplexed and (b) complexed, mesoporphyrin and the type I porphyrins in the electric field

\subsubsection{Conclusions}

Dynamic complexation theory is used to describe the nonaqueous separation of a mixture of porphyrins using brij 35 as a buffer additive. This study also provided an opportunity to demonstrate many of the theoretical aspects of binding studies discussed in the previous chapters. The equation that describes the effect of a 1:1 analyte-additive interaction on the mobility of an analyte was derived based on the general theory presented in chapter 1.2. The 1:1 model was shown to be appropriate using the methods for detecting deviations from the 1:1 
173.

model discussed in chapter 1.3. Designing the experiment according to the optimal conditions for a binding experiment, discussed in chapters 2.2 and 2.3 , minimized errors in the estimated constants. 


\subsection{References}

(1) Walbroehl, Y.; Jorgenson, J. W. J. Chromatogr. 1984, 315, 135-43.

(2) Kenndler, E.; Gassner, B. Anal. Chem. 1990, 62, 431-6.

(3) Chiari, M.; Kenndler, E. J. Chromatogr. 1995, 716, 303-9.

(4) Salimi-Moosavi, H.; Cassidy, R. M. Anal. Chem. 1995, 67, 1067-73.

(5) Sahota, R. S.; Khaledi, M. G. Anal. Chem. 1994, 66, 1141-6.

(6) Tomlinson, A. J.; Benson, L. M.; Gorrod, J. W.; Naylor, S. J. Chromatogr. B 1994, 657, 373-81.

(7) Bjørnsdottir, I.; Hansen, S. H. J. Chromatogr. 1995, 711, 313-22.

(8) Bjørnsdottir, I.; Terabe, S.; Hansen, S. H. Seventh International Symposium on High Performance Capillary Electrophoresis (HPCE '96), 1996; poster 92.

(9) Bjørnsdottir, I.; Hansen, S. H.; Terabe, S. J. Chromatogr. A 1996, 745, 37-44.

(10) Lu, W. Z.; Poon, G. K.; Carmichael, P. L.; Cole, R. B. Anal. Chem. 1996, 68, 668-74.

(11) Salimi-Moosavi, H.; Cassidy, R. M. Anal. Chem. 1996, 68, 293-9.

(12) Lin, C. E.; Chiou, W. C.; Lin, W. C. J. Chromatogr. A 1996, 723, $189-95$.

(13) Wright, P. B.; Lister, A., S.; Dorsey, J. G. Anal. Chem. 1997, 69, 3251-9.

(14) Valkó, I. E.; Sirén, H.; Riekkola, M.-L. LC-GC 1997, 15, 560-7.

(15) Hansen, S. H.; Tjornelund, J.; Bjornsdottir, I. Tr. Anal. Chem. 1996, 15, 175-80.

(16) Krestov, G. A.; Novosyolov, N. P.; Perelygin, I. S.; Kolker, A. M.; Safonova, L. P.; Ovchinnikova, V. D.; Trostin, V. N. Ionic Solvation, 1 ed.; Ellis Horwood: New York, 1994.

(17) Weast, R. C.; Astle, M. J.; Beyer, W. H. CRC Handbook of Chemistry and Physics, 68 ed.; CRC Press, Inc.: Boca Raton, 1987.

(18) Marcus, Y. Ion Solvation; John Wiley \& Sons Limited: New York, 1985.

(19) Mayer, U. Pure \& Appl. Chem. 1979, 51, 1697-712.

(20) Evans, D. F.; Wennerström, H. The Colloidal Domain: Where Physics, Chemistry, Biology and Technology Meet; VCH Publishers: New York, 1994.

(21) Rico, I.; Lattes, A. J. Phys. Chem. 1986, 90, 5870-2.

(22) Wang, F.; Khaledi, M. G. Anal. Chem. 1996, 68, 3460-7.

(23) Valkó, I. E.; Sirén, H.; Riekkola, M. L. Chromatographia 1996, 43, 242-6.

(24) Valkó, I. E.; Sirén, H.; Riekkola, M. L. J. Chromatogr. A 1996, 737, $263-72$.

(25) Valkó, I. E.; Sirén, H.; Riekkola, M.-L. Electrophoresis 1997, 18, 919-23.

(26) Laidler, K. J.; Meiser, J. H. Physical Chemistry; The Benjamin/Cummings Publishing Company Inc.: Menlo Park, CA, 1982.

(27) Burger, K. Solvation, Ionic and Complex Formation Reactions in Non-Aqueous Solvents: Experimental Methods for Their Investigation; Elsevier Scientific Publishing Company: New York, 1983.

(28) Kranack, A. R. MSc. Thesis, University of British Columbia, Vancouver, 1998.

(29) Walbroehl, Y.; Jorgenson, J. W. Anal. Chem. 1986, 58, 479-81.

(30) Gutmann, V. Electrochim. Acta. 1976, 21, 661-70. 
(31) Marcus, Y. J. Soln. Chem. 1984, 13, 599-624.

(32) Mori, Y.; Ueno, K.; Umeda, T. J. Chromatogr. A 1997, 757, 328-32.

(33) Okada, T. J. Chromatogr. A 1995, 695, 309-17.

(34) Salimi-Moosavi, H.; Cassidy, R. M. J. Chromatogr. A 1996, 749, 279-86.

(35) Miller, J. L.; Khaledi, M. G.; Shea, D. Anal. Chem. 1997, 69, 1223-9.

(36) Stalcup, A. M.; Gahm, K. H. J. Microcol. Sep. 1996, 8, 145-50.

(37) Dougherty, T. J. Photochem. Photobiol. 1987, 45, 879-89.

(38) Dougherty, T. J. Photochem. Photobiol. 1987, 46, 569-73.

(39) Bonnett, R. Chem. Soc. Rev. 1995, 24, 19-33.

(40) Lipson, R. L.; Baldes, E. D.; Olsen, A. M. J. Natl. Cancer Inst. 1961, 26, 1-11.

(41) Byrne, C. J.; Marshallsay, L. V.; Ward, A. D. J. Photochem. Photobiol. B: Biol. 1990, 6, 13-27.

(42) Byrne, C. J.; Marshallsay, L. V.; Ward, A. D. Photochem. Photobiol. 1987, 46, 575-80.

(43) Kessel, D.; Thompson, P.; Musselman, B.; Chang, C. K. Photochem. Photobiol. 1987, 46, 563-8.

(44) Owens, J. W.; Yang, L.; Adeola, G.; Robins, M.; Smith, R.; Robinson, R.; Elayan, N.; McMahon, L. J. Chromatogr. B 1995, 669, 295-309.

(45) Vernon, D. I.; Holroyd, J. A.; Stribbling, S. M.; Brown, S. B. J. Photochem. Photobiol. B: Biol. 1995, 27, 209-17.

(46) Wu, N.; Barker, G. E.; Huie, C. W. J. Chromatogr. 1994, 659, 435-42.

(47) Wu, N.; Li, B.; Sweedler, J. V. J. Liq. Chromatogr. 1994, 17, 1917-27.

(48) Chan, E. N. L.; Goodall, D. M. J. Chromatogr. 1993, 636, 171-8.

(49) Kiyohara, C.; Saitoh, K.; Suzuki, N. J. Chromatogr. 1993, 646, 397-403.

(50) Weinberger, R.; Sapp, E.; Moring, S. J. Chromatogr. 1990, 516, 271-85.

(51) Lucy, C. A.; McDonald, T. L. Anal. Chem. 1995, 67, 1074-8.

(52) Culbertson, C. T.; Jorgenson, J. W. Anal. Chem. 1994, 66, 955-62.

(53) Gregory, R. P. F. Biochemistry of Photosynthesis; Wiley Interscience: New York, 1971.

(54) Ho, C. Electron Transport and Oxygen Utilization; Elsevier Biomedical: New York, 1980.

(55) Smith, K. M.; Fuhrhop, J.-H. Laboratory Methods; Elsevier Scientific Publishing: Amsterdam, 1975.

(56) Eales, L. In Porphyrins; Dolphin, D., Ed.; Academic Press: New York, 1979; Vol. VI, pp 285302.

(57) Meguro, K.; Ueno, M.; Esumi, K. In Nonionic Surfactants; Schick, M. J., Ed.; Marcel Dekker, Inc.: New York, 1987.

(58) Ege, S. Organic Chemistry; D. C. Heath and Company: Toronto, 1989.

(59) Connors, K. A. Binding Constants - The Measurement of Molecular Complex Stability; John Wiley \& Sons: Toronto, 1987. 


\section{Concluding Remarks}

The material presented in this thesis provides the foundation required to study analyteadditive interactions in CE. Part 1 described a general theory that allows complicated equilibria, including multiple additive and multiple stoichiometry interactions, to be studied in CE. The theory was also shown to be generally applicable in separation science. Part 2 demonstrated the effect of experimental conditions on binding constant estimates. This is of significant importance if $\mathrm{CE}$ is going to be used as a tool to study chemical interactions. Part 3 discussed analyte-additive interactions in the quickly growing field of nonaqueous CE. This last section also provided an opportunity to demonstrate a practical application of the largely theoretical discussions presented in earlier chapters.

Future work should focus on the application of the fundamentals presented in this thesis. The theory presented in Part 1 should be useful in studying biological systems, which often involve multiple ligand or multiple stoichiometry interactions. The general theory will also be helpful in describing the complicated equilibria that are common in nonaqueous CE. As described in chapter 3.1, electrostatic and electron-transfer interactions between the analyte and various constituents of the buffer are more significant in nonaqueous solvents than in water. A better understanding of the effect of these "secondary" interactions on analyte mobility is needed.

The effect of experimental conditions on the reliability of binding constant estimates needs to be explored further. Although the additive concentration range and the maximum response range should be the most important variables, others need to be studied. The most important of these is probably the spacing of the data. In the simulations presented in this thesis, data was collected for additive concentrations that increased linearly. As mentioned earlier, when the data only covered the upper portion of the binding isotherm, most of the curvature in the isotherm was concentrated on several data points. It may be more reasonable to choose data 
points that are more evenly spaced over the curve of the isotherm. Other conditions that need to be tested include the error in the free mobility measurement, the number of data points and the effect of replicate measurements. Another consideration is whether the free mobility should be measured independently or included in the nonlinear regression. When using the traditional nonlinear regression, the value of the free mobility is subtracted from every data point. This may put undue emphasis on the free mobility measurement.

An important investigation that has been missing from binding studies to date has been a direct comparison of the constants measured using $\mathrm{CE}$ with constants measured using other methods. This is needed if CE is going to be used as a validated tool in the study of solution equilibria. It will also be important to determine what types of interactions are actually being measured in CE. However, care should be taken to use similar experimental conditions (e.g. solvent, ionic strength, background electrolytes, temperature, etc.) because the measured constants are often dependent on these variables. 


\section{Glossary}

acceptor number (AN): An empirical scale developed to estimate the relative electron acceptor properties of different solvents.

CE: capillary electrophoresis

CEC: capillary electrochromatography

cohesion energy density $\left(P_{S}\right)$ : The amount of energy required to break all of the solventsolvent interactions in a certain volume of solvent. Useful in estimating the strength of hydrophobic interactions in different solvents.

critical micelle concentration $(\mathrm{cmc})$ : The concentration at which individual surfactant molecules begin to form aggregates.

dengsu concentration: The concentration of an additive where the addition of a second additive, that does interact with the analyte, does not modify the analyte mobility.

dengsu point: The common intersection of the isotherms in a profile plot caused by the presence of a dengsu concentration.

dielectric: A nonconducting material that contains few or no free electrons and can support electrical stresses.

dielectric constant $(\varepsilon)$ : For a given substance, the ratio of the capacity of a condenser (capacitor) with that substance as a dielectric to the capacity of the same condensor with a vacuum for a dielectric. Also referred to as the relative permittivity.

donor number (DN): An empirical scale that estimates the relative donacity of different solvents.

electroosmotic flow (EOF): A bulk flow caused by the presence of an electric bilayer at the capillary surface.

electrophoretic mobility $\left(\mu_{\mathrm{ep}}\right)$ : The mobility of an individual ion due to the presence of an electric field.

FFF: field flow fractionation

$\gamma$. The separation factor defined as the ratio of the average linear velocities of two analytes.

Krafft temperature: The temperature at which the maximum solubility of a surfactant is equal to its $\mathrm{cmc}$. The minimum temperature at which a surfactant can form micelles.

MECC: micellar electrokinetic capillary chromatography

mobility $(\mu)$ : The linear velocity of an ion in an electric field divided by the electric field strength. Mobility is independent of the electric field strength.

v: A correction factor that normalizes the mobility to conditions where the additive concentration approaches zero. Allows the equilibria to be studied independently of other factors that may be affected by the additive.

$\zeta$-potential: Caused by the formation of an electric double layer at a solid-liquid interface. The potential difference between the Stern layer and the bulk solution. The electroosmotic mobility is directly proportional to the $\zeta$-potential. 


\section{Appendix I: Nonlinear Least-Squares Analysis}

\section{A.1 Curve Fitting}

The following sections describe the least-squares fitting procedure and its application to the equations presented in this thesis. When examining an experimental data set, it is often useful to compare the data to an empirical or theoretical equation (F) in order to describe trends in the data or enable the prediction of future measurements under different conditions. The function $\mathrm{F}$ can be written generally as:

$\mathrm{F}=\mathrm{f}(x, y, z, \ldots, \mathrm{a}, \mathrm{b}, \mathrm{c}, \ldots)=0$

where $x, y, z$, etc. are variables and $\mathrm{a}, \mathrm{b}, \mathrm{c}$, etc. are the coefficients of the equation. In the regression procedure the coefficients of F are varied until the equation best "fits" the data. The sum of squares of the residuals are used to determine how well a certain equation "fits" a data set. The residual of a data point $i$ is defined as:

$e_{y i}=Y_{i}-y_{i}$

where $e_{y i}$ is the residual in point $i, Y_{i}$ is the observed value and $y_{i}$ is the value calculated from the regression line. Because there are data above and below the regression line, some points give positive residuals while others give negative residuals. In order to minimize the overall magnitude of the residuals, the sum of the squares of the residuals $(\mathrm{S})$ is minimized. The sum of the squares of the residuals is given by:

$\mathrm{S}=\sum \mathrm{w}_{\mathrm{yi}} \mathrm{e}_{\mathrm{yi}}^{2}$

where $w_{y i}$ is the weight associated with point $i$. The values of the coefficients $(a, b, c, \ldots)$ that minimize the sum squares of the residuals for $\mathrm{F}$ can be found by solving the set of normal equations given by ${ }^{(1-4)}$ : 


$$
\begin{aligned}
& \sum w_{i} F_{a}^{i} F_{a}^{i} A+\sum w_{i} F_{a}^{i} F_{b}^{i} B+\sum w_{i} F_{a}^{i} F_{c}^{i} \mathrm{C}+\ldots=\sum w_{i} F_{a}^{i} F_{0}^{i} \\
& \sum w_{i} F_{b}^{i} F_{a}^{i} A+\sum w_{i} F_{b}^{i} F_{b}^{i} B+\sum w_{i} F_{b}^{i} F_{c}^{i} \mathrm{C}+\ldots=\sum w_{i} F_{b}^{i} F_{0}^{i} \\
& \sum w_{i} F_{c}^{i} F_{a}^{i} A+\sum w_{i} F_{c}^{i} F_{b}^{i} B+\sum w_{i} F_{c}^{i} F_{c}^{i} C+\ldots=\sum w_{i} F_{c}^{i} F_{0}^{i}
\end{aligned}
$$

where $F_{a}, F_{b}$, and $F_{c}$ are the partial derivatives respect to the coefficients $a, b$, and $c$, respectively; $\mathrm{A}, \mathrm{B}$, and $\mathrm{C}$ are defined as:

$$
\begin{aligned}
& A=a_{0}-a \\
& B=b_{0}-b \\
& C=c_{0}-c
\end{aligned}
$$

where $a_{0}, b_{0}$, and $c_{0}$ are the initial estimates of the coefficients $a, b$, and $c . F_{0}$ is the value of $F$ determined using the initial estimates of the coefficients:

$\mathrm{F}_{0}=\mathrm{F}\left(x, y, z, \ldots, \mathrm{a}_{0}, \mathrm{~b}_{0}, \mathrm{c}_{0}, \ldots\right)$

The coefficients (i.e. a, b, c, etc.) can be solved precisely if the function $F$ is linear. If the function is nonlinear, the initial estimates can not be eliminated from the normal equations. Therefore an iterative process, where initial estimates of the constants are used to make refined estimates of the constants, is required.

The equation used to describe the effect of an additive (that interacts with a 1:1 stoichiometry) on the mobility of an analyte (eq 1.3.14) takes the form of a rectangular hyperbola:

$$
y=\frac{x}{b+a x}
$$

where $\mathrm{y}=\left(v \mu_{\mathrm{ep}}^{\mathrm{A}}-\mu_{\mathrm{ep}, \mathrm{A}}\right), x=[\mathrm{C}]$, and:

$$
\mathrm{b}=\frac{1}{\left(\mu_{\mathrm{ep}, \mathrm{AC}}-\mu_{\mathrm{ep}, \mathrm{A}}\right)}
$$




$$
\mathrm{a}=\frac{\mathrm{K}_{\mathrm{AC}}}{\left(\mu_{\mathrm{ep}, \mathrm{AC}}-\mu_{\mathrm{ep}, \mathrm{A}}\right)}
$$

The functions and partial derivatives of eq A.9 are:

$$
\begin{aligned}
& \mathrm{F}=y-\frac{x}{\mathrm{~b}+\mathrm{a} x} \\
& \mathrm{~F}_{\mathrm{a}}=\frac{\partial \mathrm{F}}{\partial \mathrm{a}}=\frac{x^{2}}{(\mathrm{~b}+\mathrm{a} x)^{2}} \\
& \mathrm{~F}_{\mathrm{b}}=\frac{\partial \mathrm{F}}{\partial \mathrm{b}}=\frac{x}{(\mathrm{~b}+\mathrm{a} x)^{2}}
\end{aligned}
$$

Inserting eqs A.12 to A.14 into eq A.4 gives the normal set of equations for the nonlinear, leastsquares fit of a rectangular hyperbola:

$$
\begin{aligned}
& \sum \frac{\mathrm{w} x^{4}}{\mathrm{z}^{4}} \mathrm{~A}+\sum \frac{\mathrm{w} x^{3}}{\mathrm{z}^{4}} \mathrm{~B}=\sum\left(\frac{\mathrm{w} x^{2} y}{\mathrm{z}^{2}}+\frac{\mathrm{w} x^{3}}{\mathrm{z}^{3}}\right) \\
& \sum \frac{\mathrm{w} x^{3}}{\mathrm{z}^{4}} \mathrm{~A}+\sum \frac{\mathrm{w} x^{2}}{\mathrm{z}^{4}} \mathrm{~B}=\sum\left(\frac{\mathrm{w} x y}{\mathrm{z}^{2}}+\frac{\mathrm{w} x^{2}}{\mathrm{z}^{3}}\right)
\end{aligned}
$$

where $\mathrm{z}=\mathrm{b}_{0}+\mathrm{a}_{0} x$. Equation A.15 takes the form

$$
\mathrm{MP}=\mathrm{Q}
$$

where:

$$
\begin{aligned}
& \mathrm{M}=\left[\begin{array}{cc}
\sum \frac{\mathrm{w} x^{4}}{\mathrm{z}^{4}} & \sum \frac{\mathrm{w} x^{3}}{\mathrm{z}^{4}} \\
\sum \frac{\mathrm{w} x^{3}}{\mathrm{z}^{4}} & \sum \frac{\mathrm{w} x^{2}}{\mathrm{z}^{4}}
\end{array}\right] \\
& \mathrm{P}=\left[\begin{array}{l}
\mathrm{A} \\
\mathrm{B}
\end{array}\right]
\end{aligned}
$$


182.

$$
\mathrm{Q}=\left[\begin{array}{c}
\sum\left(\frac{\mathrm{w} x^{2} y}{\mathrm{z}^{2}}+\frac{\mathrm{w} x^{3}}{\mathrm{z}^{3}}\right) \\
\sum\left(\frac{\mathrm{w} x y}{\mathrm{z}^{2}}+\frac{\mathrm{w} x^{2}}{\mathrm{z}^{3}}\right)
\end{array}\right]
$$

A and B can be solved by rearranging eq A.16 to:

$\mathrm{P}=\mathrm{M}^{-1} \mathrm{Q}$

The values for A and B can be used with the initial coefficient estimates $\left(a_{0}\right.$ and $\left.b_{0}\right)$ to solve for the revised estimates of $a$ and $b$. The revised estimates then become the initial parameters for the next iteration. This procedure is continued until the parameter estimates converge.

A similar strategy was used to perform the nonlinear least squares regression of the equation that accounted for both first and second order analyte-additive interactions (eq 1.3.13). Equation 1.3 .13 can be written as:

$$
y=\frac{a x+b x^{2}}{1+c x+d x^{2}}
$$

The expressions needed to derive the normal equations are:

$$
\begin{aligned}
& \mathrm{F}=y-\frac{a x+b x^{2}}{1+c x+d x^{2}} \\
& F_{a}=\frac{\partial F}{\partial a}=\frac{-x}{\left(1+c x+d x^{2}\right)} \\
& F_{b}=\frac{\partial F}{\partial b}=\frac{-x^{2}}{\left(1+c x+d x^{2}\right)} \\
& F_{c}=\frac{\partial F}{\partial c}=\frac{x\left(a x+b x^{2}\right)}{\left(1+c x+d x^{2}\right)^{2}} \\
& F_{d}=\frac{\partial F}{\partial d}=\frac{x^{2}\left(a x+b x^{2}\right)}{\left(1+c x+d x^{2}\right)^{2}}
\end{aligned}
$$


183.

The matrices that make up the normal equations for eq A.21 are:

$\mathrm{M}=\left[\begin{array}{cccc}\sum \frac{x^{2}}{\mathrm{n}^{2}} & \sum \frac{x^{3}}{\mathrm{n}^{2}} & \sum \frac{-x^{2} \mathrm{~m}}{\mathrm{n}^{3}} & \sum \frac{-x^{3} \mathrm{~m}}{\mathrm{n}^{3}} \\ \sum \frac{x^{3}}{\mathrm{n}^{2}} & \sum \frac{x^{4}}{\mathrm{n}^{2}} & \sum \frac{-x^{3} \mathrm{~m}}{\mathrm{n}^{3}} & \sum \frac{-x^{4} \mathrm{~m}}{\mathrm{n}^{3}} \\ \sum \frac{-x^{2} \mathrm{~m}}{\mathrm{n}^{3}} & \sum \frac{-x^{3} \mathrm{~m}}{\mathrm{n}^{3}} & \sum \frac{x^{2} \mathrm{~m}^{2}}{\mathrm{n}^{4}} & \sum \frac{x^{3} \mathrm{~m}^{2}}{\mathrm{n}^{4}} \\ \sum \frac{-x^{3} \mathrm{~m}}{\mathrm{n}^{3}} & \sum \frac{-x^{4} \mathrm{~m}}{\mathrm{n}^{3}} & \sum \frac{x^{3} \mathrm{~m}^{2}}{\mathrm{n}^{4}} & \sum \frac{x^{4} \mathrm{~m}^{2}}{\mathrm{n}^{4}}\end{array}\right]$

$P=\left[\begin{array}{l}A \\ B \\ C \\ D\end{array}\right]$

$\mathrm{Q}=\left[\begin{array}{c}\sum\left(\frac{x \mathrm{~m}}{\mathrm{n}^{2}}-\frac{x y}{\mathrm{n}}\right) \\ \sum\left(\frac{x^{2} \mathrm{~m}}{\mathrm{n}^{2}}-\frac{x^{2} y}{\mathrm{n}}\right) \\ \sum\left(\frac{x y \mathrm{~m}}{\mathrm{n}^{2}}-\frac{x \mathrm{~m}^{2}}{\mathrm{n}^{3}}\right) \\ \sum\left(\frac{x^{2} y \mathrm{~m}}{\mathrm{n}^{2}}-\frac{x^{2} \mathrm{~m}^{2}}{\mathrm{n}^{3}}\right)\end{array}\right]$

where $\mathrm{m}$ and $\mathrm{n}$ are given by:

$$
\begin{aligned}
& \mathrm{m}=\mathrm{a}_{0} x+\mathrm{b}_{0} x^{2} \\
& \mathrm{n}=1+\mathrm{c}_{0} x+\mathrm{d}_{0} x^{2}
\end{aligned}
$$

The parameters can be found by solving for $\mathrm{P}$ in: $\mathrm{P}=\mathrm{M}^{-1} \mathrm{Q}$.

\section{A.2 Weighting}

Differences in the errors contributed to the overall function by individual data points can be accounted for using weighted regressions. The "w" in the normal equations indicate the 
184.

relative significance that should be given to each data point when solving for the coefficients. The amount of weight given to a certain point $\left(\mathrm{w}_{\mathrm{i}}\right)$ is inversely proportional to the variance contributed to the overall equation by that point $\left(\sigma_{\mathrm{F}, \mathrm{i}}^{2}\right)$ :

$$
\mathrm{w}_{\mathrm{i}}=\frac{\mathrm{k}}{\sigma_{\mathrm{F}, \mathrm{i}}^{2}}
$$

where $\mathrm{k}$ is a constant. If the variance contributed by each point is equal, then $\mathrm{w}_{\mathrm{i}}$ is equal for all of the data points, and no weighting is required. This assumption is often made without basis. Weighting is especially important when using the linear transformations of the binding isotherm. Even if the error in each of the measured data points is equal, the errors in the values are often changed during the transformation.

Using propagation of error, it can be shown that the errors in the $x$ and $y$ variables are transformed to the error in the function according to ${ }^{(1-4)}$ :

$$
\sigma_{\mathrm{F}}^{2}=\mathrm{F}_{x} \mathrm{~F}_{x} \sigma_{x}^{2}+\mathrm{F}_{y} \mathrm{~F}_{y} \sigma_{y}^{2}+2 \mathrm{~F}_{x} \mathrm{~F}_{y} \sigma_{x y}
$$

where $\sigma_{\mathrm{F}}^{2}$ is the variance in the function $\mathrm{F}, \sigma_{x}^{2}$ is the variance in the value for $x, \sigma_{y}^{2}$ is the variance in the value for $y, \sigma_{x y}$ is the covariance between the values $x$ and $y, \mathrm{~F}_{x}$ is the partial derivative with respect to $x$, and $\mathrm{F}_{y}$ is the partial derivative with respect to $y$. If the errors in the variables ( $x$ and $y$ ) are independent, the covariance is zero, allowing eq A.33 to be written as:

$$
\sigma_{\mathrm{F}}^{2}=\mathrm{F}_{x} \mathrm{~F}_{x} \sigma_{x}^{2}+\mathrm{F}_{y} \mathrm{~F}_{y} \sigma_{y}^{2}
$$

The equation for the double reciprocal plot (see Table 2.1.1) takes the form:

$$
\frac{1}{y}=\frac{\mathrm{m}}{x}+\mathrm{n}=y^{\prime}=\mathrm{m} x^{\prime}+\mathrm{n}
$$

where $y^{\prime}$ and $x^{\prime}$ are the transformed variables. Writing eq A.35 in terms of the function F gives: 
$\mathrm{F}=\frac{1}{y}-\frac{\mathrm{m}}{x}-\mathrm{n}$

The partial derivatives of eq A.36 are:

$\mathrm{F}_{x}=\frac{\mathrm{m}}{x^{2}}$

$\mathrm{F}_{y}=\frac{1}{y^{2}}$

Inserting eqs A.37 and A.38 into eq A.34 gives:

$\sigma_{y^{\prime}}^{2}=\frac{m^{2} \sigma_{x}^{2}}{x^{4}}+\frac{\sigma_{y}^{2}}{y^{4}}$

If $\sigma_{x}^{2} \ll \sigma_{y}^{2}$ then:

$\sigma_{y^{\prime}}^{2}=\frac{\sigma_{y}^{2}}{y^{4}}$

This simplifies the weighting process because the weight associated with each point is no longer influenced by the parameter $\mathrm{m}$.

The same procedure can be used for the $y$-reciprocal transformation:

$$
\begin{aligned}
& \frac{x}{y}=\mathrm{n} x+\mathrm{m}=y^{\prime}=\mathrm{m} x^{\prime}+\mathrm{n} \\
& \mathrm{F}=\frac{x}{y}-\mathrm{n} x-\mathrm{m} \\
& \mathrm{F}_{x}=\frac{1}{y}-\mathrm{n} \\
& \mathrm{F}_{y}=-\frac{x}{y^{2}}
\end{aligned}
$$


Inserting eqs A.43 and A.42 into eq A.34 gives:

$$
\sigma_{y^{\prime}}^{2}=\sigma_{x}^{2}\left(\frac{1}{y^{2}}+\mathrm{n}^{2}\right)+\frac{x^{2} \sigma_{y}^{2}}{y^{4}}
$$

Making the assumption $\sigma_{x}^{2} \ll \sigma_{y}^{2}$ gives:

$$
\sigma_{y^{\prime}}^{2}=\frac{x^{2} \sigma_{y}^{2}}{y^{4}}
$$

The function and partial derivatives of the $x$-reciprocal transformation are:

$$
\begin{aligned}
& \frac{y}{x}=-\frac{\mathrm{n}}{\mathrm{m}} y+\frac{1}{\mathrm{~m}}=y^{\prime}=\mathrm{m} x^{\prime}+\mathrm{n} \\
& \mathrm{F}=\frac{y}{x}+\frac{\mathrm{n}}{\mathrm{m}} y-\frac{1}{\mathrm{~m}} \\
& \mathrm{~F}_{x}=-\frac{y}{x^{2}} \\
& \mathrm{~F}_{y}=\frac{1}{x}+\frac{\mathrm{n}}{\mathrm{m}}
\end{aligned}
$$

Inserting eqs A.49 and A.50 into eq A.34 gives:

$$
\sigma_{y^{\prime}}^{2}=\frac{y^{2} \sigma_{x}^{2}}{x^{4}}+\left(\frac{1}{x}+\frac{\mathrm{n}}{\mathrm{m}}\right)^{2} \sigma_{y}^{2}
$$

Making the assumption $\sigma_{x}^{2} \ll \sigma_{y}^{2}$ gives:

$$
\sigma_{y^{\prime}}^{2}=\left(\frac{1}{x}+\frac{\mathrm{n}}{\mathrm{m}}\right)^{2} \sigma_{y}^{2}
$$

The weighting function for the $x$-reciprocal plot is dependent on the coefficients ( $m$ and $n$ ). An iterative process is therefore necessary, where an initial estimate of the coefficients needs to be made before the weight for each data point can be determined. Once the weights are calculated, 
a revised estimate of the parameters can be made. This process should be continued until the coefficient estimates converge.

The process used to determine the weighting functions of the linear transformations also applies to the nonlinear form of the binding isotherm:

$$
y=\frac{x}{m+n x}
$$

$$
\mathrm{F}=\mathrm{y}-\frac{x}{\mathrm{~m}+\mathrm{nx}}
$$

$$
\mathrm{F}_{x}=\frac{-\mathrm{m}}{(\mathrm{m}+\mathrm{nx})^{2}}
$$

$\mathrm{F}_{y}=1$

Inserting eqs A.55 and A.56 into eq A.34 gives:

$$
\sigma_{y^{\prime}}^{2}=\frac{m^{2} \sigma_{x}^{2}}{(m+n x)^{4}}+\sigma_{y}^{2}
$$

If $\sigma_{x}^{2} \ll \sigma_{y}^{2}$ then eq A.57 reduces to:

$$
\sigma_{y^{\prime}}^{2}=\sigma_{y}^{2}
$$

Therefore, when using the nonlinear form of the equation, the variance contributed by an individual point is equal to the variance of that point. No weighting is necessary if the error in all of the data points is equal.

The weight given to a certain point indicates the significance of that point in relation to the other points. Therefore, it is the relative values of the weights that are important, not their absolute values. This simplifies the calculation of the weight associated with each point. The variance contributed by each point is related to the variance of the data point. In order to 
188.

calculate the absolute values of the weights, the absolute value of the variance of that data point would need to be measured. The relative variances of the points are easier to estimate than the absolute values.

The values of the weights can be normalized according to:

$$
\mathrm{w}_{\mathrm{i}}=\frac{\mathrm{n} / \sigma_{y^{\prime}, \mathrm{i}}^{2}}{\sum\left(1 / \sigma_{y^{\prime}, \mathrm{i}}^{2}\right)}
$$

where $w_{i}$ is the weight associated with point $i, n$ is the number of data points, and $\sigma_{y^{\prime}, i}^{2}$ is the relative variance contributed by point $i$ to the overall equation. When normalized this way, all of the data points will have weights of one if $\sigma_{y^{\prime}, i}^{2}$ is equal for all of the points.

\section{A.3 Estimating the Error in the Coefficients}

Solving the normal equations (eq A.4) using matrices simplifies the procedure for estimating the error in the coefficients. For an equation with three coefficients, the matrix $\mathrm{M}$ can be written generally as:

$$
M=\left[\begin{array}{lll}
F_{a} F_{a} & F_{a} F_{b} & F_{a} F_{c} \\
F_{b} F_{a} & F_{b} F_{b} & F_{b} F_{c} \\
F_{c} F_{a} & F_{c} F_{b} & F_{c} F_{c}
\end{array}\right]
$$

There will be an inverse matrix of $M$ where the matrix elements are indexed according to:

$$
\mathbf{M}^{-1}=\left[\begin{array}{ccc}
a a & a b & a c \\
b a & b b & b c \\
c a & c b & c c
\end{array}\right]
$$

The diagonal matrix elements of $\mathbf{M}^{-1}$ give the variances of the coefficients:

$$
a a=\frac{\sigma_{\mathrm{a}}^{2}}{\sigma^{2}}
$$




$$
b b=\frac{\sigma_{\mathrm{b}}^{2}}{\sigma^{2}}
$$

$$
c c=\frac{\sigma_{c}^{2}}{\sigma^{2}}
$$

where $\sigma_{\mathrm{a}}^{2}, \sigma_{\mathrm{b}}^{2}$, and $\sigma_{\mathrm{c}}^{2}$ are the variances of the coefficients and $\sigma^{2}$ is the variance of the

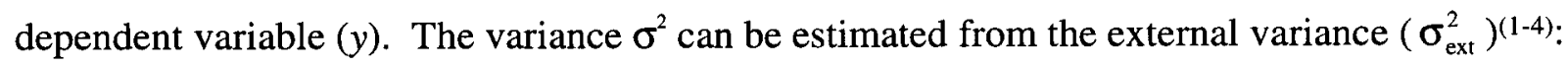

$$
\sigma^{2} \approx \sigma_{\mathrm{ext}}^{2}=\frac{\sum \mathrm{we}^{2}}{\mathrm{n}-\mathrm{p}}
$$

where $\mathrm{e}$ is the sum of the squares of the residuals, $\mathrm{n}$ is the number of data points, and $\mathrm{p}$ is the number of coefficients. The off-diagonal elements of the inverse matrix give the covariances of the coefficients:

$$
\begin{aligned}
& a b=b a=\frac{\sigma_{\mathrm{ab}}}{\sigma^{2}} \\
& a c=c a=\frac{\sigma_{\mathrm{ac}}}{\sigma^{2}} \\
& b c=c b=\frac{\sigma_{\mathrm{bc}}}{\sigma^{2}}
\end{aligned}
$$

The covariances are important if the coefficients are to be used in further calculations. For example, when performing a nonlinear regression according to eq A.9, the binding constant is equal to a divided by $b$. According to the propagation of error, the error in the binding constant $\left(\sigma_{\mathrm{K}}^{2}\right)$ is given by:

$$
\sigma_{K}^{2}=F_{a} F_{a} \sigma_{a}^{2}+F_{b} F_{b} \sigma_{b}^{2}+2 F_{a} F_{b} \sigma_{a b}
$$

Because the errors in the parameters are generally not independent, the third term in eq A.69 is not zero. Inserting the partial derivatives into eq A.69 gives: 
$\sigma_{K}^{2}=K^{2}\left(\frac{\sigma_{a}^{2}}{a^{2}}+\frac{\sigma_{b}^{2}}{b^{2}}+\frac{\sigma_{a b}}{a b}\right)$

Equation A.70 allows the error in $\mathrm{K}$ to be determined from the error in the coefficients $\mathrm{a}$ and $\mathrm{b}$.

\section{A.4 References}

(1) Deming, W. E. Phil. Mag. 1931, 11, 146-58.

(2) Deming, W. E. Phil. Mag. 1934, 17, 804-29.

(3) Deming, W. E. Phil. Mag. 1935, 19, 389-402.

(4) Connors, K. A. Binding Constants - The Measurement of Molecular Complex Stability; John Wiley \& Sons: Toronto, 1987. 